Florida International University FIU Digital Commons

\title{
In Situ Arsenic Speciation using Surface-enhanced Raman Spectroscopy
}

Mingwei Yang

Florida International University, minyang@fiu.edu

DOI: $10.25148 /$ etd.FIDC001951

Follow this and additional works at: https://digitalcommons.fiu.edu/etd

Part of the Analytical Chemistry Commons

\section{Recommended Citation}

Yang, Mingwei, "In Situ Arsenic Speciation using Surface-enhanced Raman Spectroscopy" (2017). FIU Electronic Theses and Dissertations. 3387.

https://digitalcommons.fiu.edu/etd/3387

This work is brought to you for free and open access by the University Graduate School at FIU Digital Commons. It has been accepted for inclusion in FIU Electronic Theses and Dissertations by an authorized administrator of FIU Digital Commons. For more information, please contact dcc@fiu.edu. 


\title{
FLORIDA INTERNATIONAL UNIVERSITY
}

Miami, Florida

\section{IN SITU ARSENIC SPECIATION USING SURFACE-ENHANCED RAMAN SPECTROSCOPY}

\author{
A dissertation submitted in partial fulfillment of \\ the requirements for the degree of \\ DOCTOR OF PHILOSOPHY
}

in

CHEMISTRY

by

Mingwei Yang

2017 
To: Dean Michael R. Heithaus

College of Arts, Sciences and Education

This dissertation, written by Mingwei Yang, and entitled In situ Arsenic Speciation using Surface-enhanced Raman Spectroscopy, having been approved in respect to style and intellectual content, is referred to you for judgment.

We have read this dissertation and recommend that it be approved.

$\begin{array}{r}\hline \text { Yi Xiao } \\ \hline \text { Rudolf Jaffe } \\ \hline \text { Bruce McCord } \\ \hline \text { Anthony McGoron } \\ \hline \text { Yong Cai, Major Professor }\end{array}$

Date of Defense: June 302017

The dissertation of Mingwei Yang is approved.

Dean Michael R. Heithaus

College of Arts, Sciences and Education

Andrés G. Gil

Vice President for Research and Economic Development and Dean of the University Graduate School

Florida International University, 2017 


\section{ACKNOWLEDGMENTS}

I would like to express my sincere appreciation to Dr Cai-my major Professor, for providing the opportunity to work in his research group and leading me into the right pathway when I was on the wrong tracks many times. Without his patient guidance, financial support and insightful understanding of chemistry, I would not have completed this work. I am also grateful to the rest of my committee members, Dr Yi Xiao, Dr Rudolf Jaffe, Dr Bruce McCord, and Dr Anthony McGoron for their support, critical comments, and suggestions during all these meetings. Special thanks to Dr Anthony McGoron, Dr Wei-chiang Lin and Dr Yun Qian in Biomedical Engineering Department for allowing me to work on the Raman instrument and for financial support to maintain the equipment. This work would have been impossible without Raman spectroscopy.

Special thanks to Dr Guangliang Liu (our lab manager and assistant director), Dr Yuzhen Sun (Jianghan University, China) and Dr Changjun Fan (a current postdoc in our research group) for their numerous time input and critical comments and beneficial suggestions.

I am grateful to Dr Shannon Matulis and Dr Lawrence Boise from Emory University for their generous providing cell pellets, my research will be less significant without these important samples.

I also would like to thank the following persons, Dr Mebel Alexander for beneficial suggestions in theoretical computation, Dr Abuzer Kabir for the discussion of silver nanofilm fabrication, REU student Kelli Sylvers for the help with coffee ring experiments, Dr Vinay Bhardwaj for his help in the use of Raman spectroscopy, Dr 
Daniel Belisario-Lara for his help in Gaussian density functional theory training, Ya-Li Hsu for his help in NMR analysis, Dr Cassian D'Cunha from FIU HPC faculty for the technical support and FIU mass facility for their help in MS analysis.

Also I like to thank all members in Dr Cai's lab for offering a nice academic and pleasant working atmosphere from past to the present, including, Dr Shujuan Sun, Dr Cheng Zhang, Dr Na Zhu, Szabina Stice, Dionne Dickson, Ping Jiang, Wenbin Cui, Yuping Xiang, et al.

Lastly, I would like to express my deepest appreciation and gratefulness to all my family members and my fiancée Miss Yan Zhang in China, without their constant and unconditional supports, encouragements and love; this work will be pale and less meaningful. 


\title{
ABSTRACT OF THE DISSERTATION
}

IN SITU ARSENIC SPECIATION USING SURFACE-ENHANCED RAMAN

SPECTROSCOPY

\author{
by
}

Mingwei Yang

Florida International University, 2017

Miami, Florida

\section{Professor Yong Cai, Major Professor}

Arsenic (As) undergoes extensive metabolism in biological systems involving numerous metabolites with varying toxicities. It is important to obtain reliable information on arsenic speciation for understanding toxicity and relevant modes of action. Currently, popular arsenic speciation techniques, such as chromatographic/electrophoretic separation following extraction of biological samples, may induce the alternation of arsenic species during sample preparation. The present study was aimed to develop novel arsenic speciation methods for biological matrices using surface-enhanced Raman spectroscopy (SERS), which, as a rapid and non-destructive photon scattering technique. The use of silver nanoparticles with different surface coating molecules as SERS substrates permits the measurement of four common arsenicals, including arsenite (As $\left.{ }^{\mathrm{III}}\right)$, arsenate $\left(\mathrm{As}^{\mathrm{V}}\right)$, monomethylarsonic acid $\left(\mathrm{MMA}^{\mathrm{V}}\right)$ and dimethylarsinic acid $\left(\mathrm{DMA}^{\mathrm{V}}\right)$. This speciation was successfully carried out using positively charged nanoparticles, and simultaneous detection of arsenicals was achieved. Secondly, arsenic speciation using coffee ring effect-based separation and SERS detection was explored on a silver nanofilm (AgNF), which was prepared by close packing of silver nanoparticles (AgNPs) on a glass 
substrate surface. Although arsenic separation using the conventional coffee ring effect is difficult because of the limited migration distance, a halo coffee ring was successfully developed through addition of surfactants, and was shown to be capable of arsenicals separation. The surfactants introduced in the sample solution reduce the surface tension of the droplet and generate strong capillary action. Consequently, solvent in the droplet migrated into the peripheral regions and the solvated arsenicals to migrated varying distances due to their differential affinity to AgNF, resulting in a separation of arsenicals in the peripheral region of the coffee ring. Finaly, a method combining experimental Raman spectra measurements and theoretical Raman spectra simulations was developed and employed to obtain Raman spectra of important and emerging arsenic metabolites. These arsenicals include monomethylarsonous acid (MMA $\left.{ }^{\mathrm{III}}\right)$, dimethylarsinous acid $\left(\right.$ DMA $\left.^{\mathrm{III}}\right)$, dimethylmonothioarinic acid $\left(\mathrm{DMMTA}^{\mathrm{V}}\right)$, dimethyldithioarsinic acid $\left(\mathrm{DMDTA}^{\mathrm{V}}\right), \quad \mathrm{S}-\left(\right.$ Dimethylarsenic) cysteine $\left(\mathrm{DMA}^{\mathrm{III}} \mathrm{Cys}\right)$ and dimethylarsinous glutathione (DMA $\left.{ }^{\mathrm{III}} \mathrm{GS}\right)$. The fingerprint vibrational frequencies obtained here for various arsenicals, some of which have not reported previously, provide valuable information for future SERS detection of arsenicals. 


\section{TABLE OF CONTENTS}

CHAPTER

PAGE

Chapter 1 Introduction, Problem Statement, Objectives and Hypotheses ...................... 1

1.1 Relationship between arsenic and human health .................................. 2

1.2 Cellular stress from arsenic metabolism in human....................................... 8

1.3 Current analytical techniques for arsenic speciation .................................. 11

1.4 Surface-enhanced Raman spectroscopy for arsenic detection...................... 14

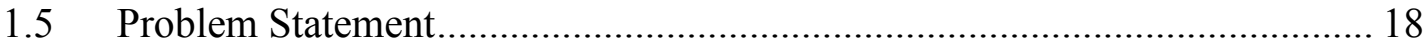

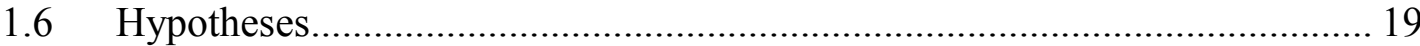

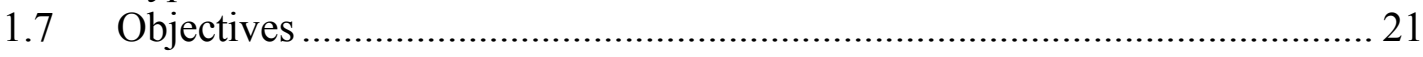

Chapter 2 Potential Application of SERS for Arsenic Speciation in Biological

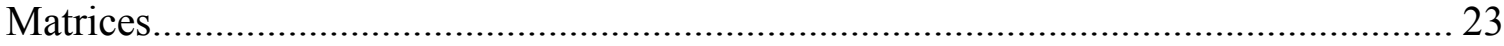

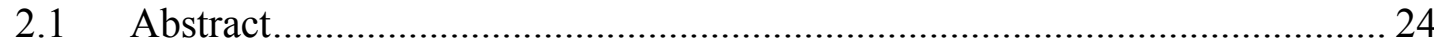

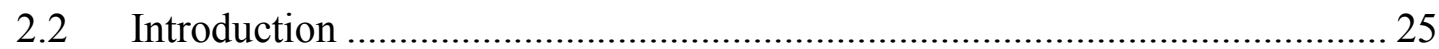

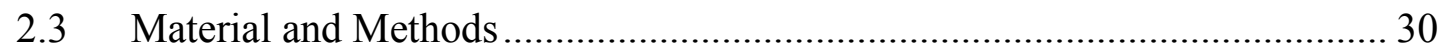

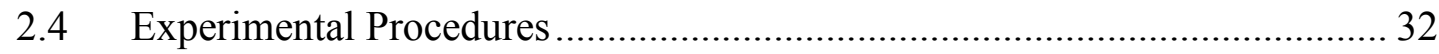

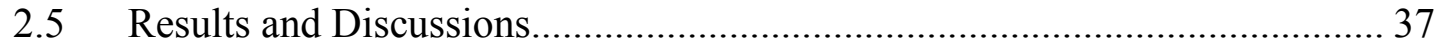

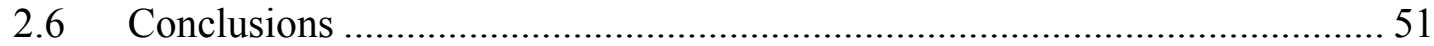

Chapter 3 Employing Coffee Ring Effect on Silver Nanofilm for Arsenic Speciation

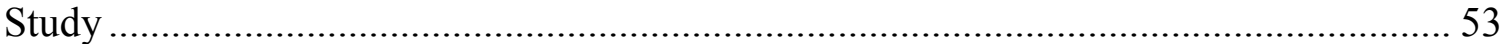

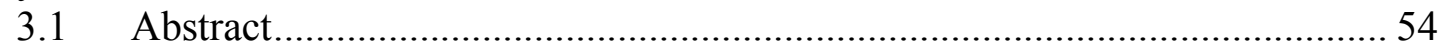

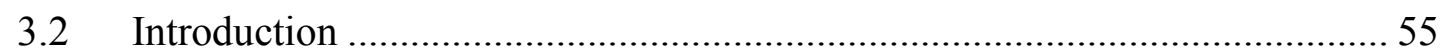

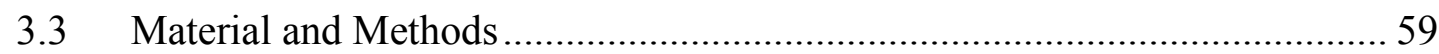

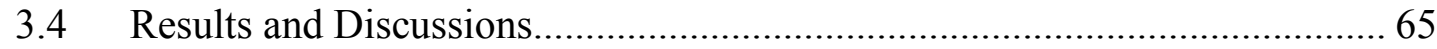

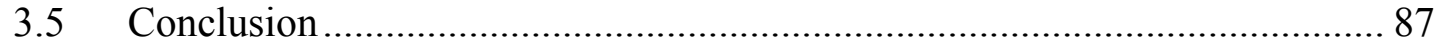

Chapter 4 Raman Spectra of Thiolated Arsenicals with Biological Importance........... 89

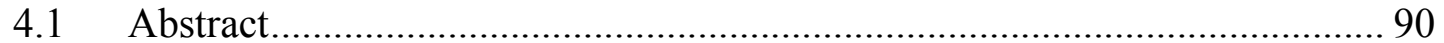

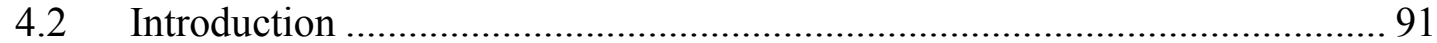

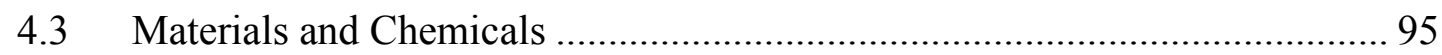

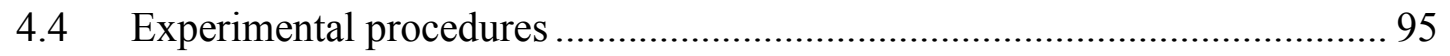

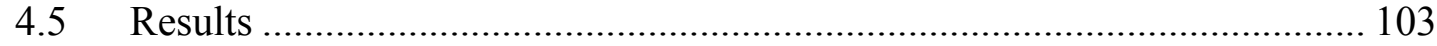

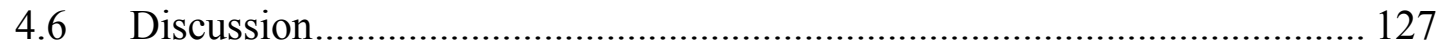

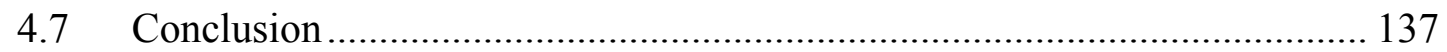

Chapter 5 Summary, Significance, and Future Research Directions......................... 138

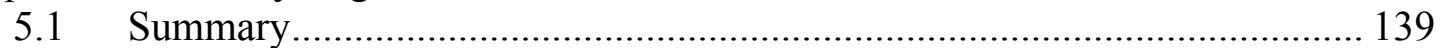

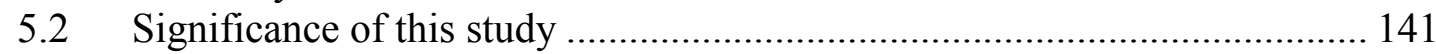

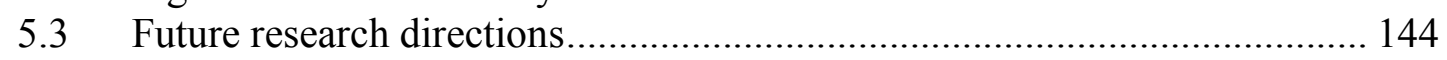

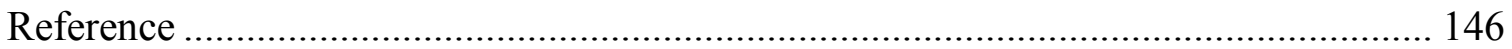




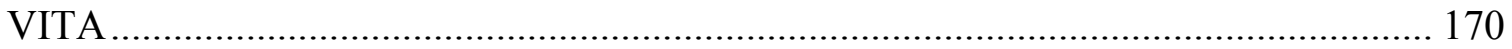




\section{LIST OF TABLES}

TABLE

PAGE

Table 2.1 Changes in deprotonation of arsenicals in different AgNPs colloidal suspensions

Table 4.1 Comparison of experimental and calculated Raman frequencies for $\mathrm{DMA}^{\mathrm{V}}$ using all basis set

Table 4.2 Comparisons of computational performance of all basis sets

Table 4.3 Comparison of all As-O bonds Raman frequencies between experimental and calculated Raman spectra

Table 4.4 Vibrational frequencies assignment for $\mathrm{MMA}^{\mathrm{III}}$ and $\mathrm{DMA}^{\mathrm{III}}$ 117

Table 4.5 Vibrational frequencies assignment for DMMTA $^{\mathrm{V}}$ and DMDTA ${ }^{\mathrm{V}}$....... 122

Table 4.6 Vibrational frequencies assignment for DMA ${ }^{\mathrm{III}} \mathrm{GS}$ and $\mathrm{DMA}^{\mathrm{III}} \mathrm{Cys}$...... 127

Table 4.7 Summary of As-O Raman frequencies............................................. 131

Table 4.8 Summary of As-C Raman frequencies ............................................. 134

Table 4.9 Summary of As-S Raman frequencies ........................................ 136 


\section{LIST OF FIGURES}

FIGURE

PAGE

Figure 2.1 The distribution of individual arsenic species in aqueous solution with different $\mathrm{pH}$ 37

Figure 2.2 Typical zeta potential and size distribution of AgNPs-Citrate (A) and AgNPs-Spermine (B). 38

Figure 2.3 SERS spectra of individual arsenic species obtained in colloidal suspensions of AgNPs-Citrate (A) and AgNPs-Spermine (B).

Figure 2.4 Adsorption of arsenic in colloidal suspensions of AgNPs-Citrate (A) and AgNPs-Spermine (B).

Figure 2.5 Arsenic concentration in the sample reservoir vs. in the filtrate receiver $(\%)$

Figure 2.6 SERS spectra of arsenic species in the cell lysate.

Figure 2.7 Changes in size (A) and zeta potential (B) of AgNPs upon interaction with cell lysate.

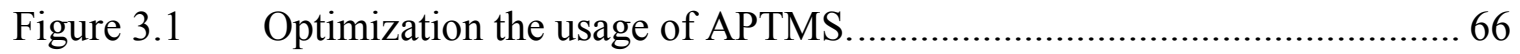

Figure 3.2 Properties of the AgNF.

Figure 3.3 Individual arsenic SERS signals on the AgNF as droplet (as black curves) and at the edge of dry film (as red curves).

Figure 3.4 Typical SERS background signals from different buffer system with or without SDS including.

Figure 3.5 The typical coffee ring signal profile of aresnicals on the AgNF

Figure 3.6 Arsenic speciation based on regular coffee ring phenomenon in different buffer solutions.

Figure 3.7 Typical halo coffee ring phenomenon as droplet and as dry film using the phosphate buffer system containing $0.05 \%$ SDS.

Figure 3.8 SERS signals along the radius of the sessile droplet containing $0.05 \%$ CTAB.

Figure 3.9 Halo coffee ring SERS signal profile of 4 arsenic species in acetic acid. 
Figure 3.10 Halo coffee ring SERS signal profile of 4 arsenic species in phosphate buffer. 83

Figure 3.11 Halo coffee ring SERS signal profile of 4 arsenic species in ammonium formate.

Figure 4.1 3D structures of arsenicals optimized by DFT ………………………... 104

Figure 4.2 Theoretical DMA ${ }^{\mathrm{V}}$ Raman spectra calculated from different basis sets....... 112

Figure 4.3 Raman spectra of $\mathrm{DMA}^{\mathrm{V}}$ obtained via experimental measurement and

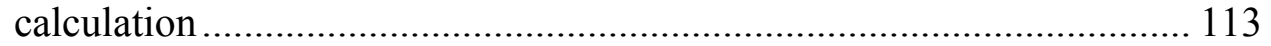

Figure 4.4 Experimental and calculated Raman spectra of $\mathrm{MMA}^{\mathrm{III}}(\mathrm{A})$ and DMA ${ }^{\mathrm{III}}$

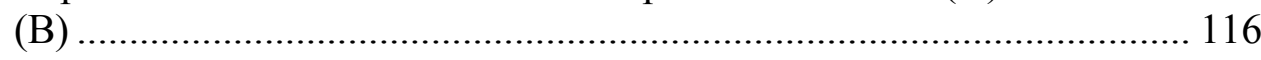

Figure 4.5 HPLC-ICP-MS chromatograms of arsenic standards and MMA synthesized in the laboratory showing the identity and purity

Figure 4.6 Proton NMR spectra for the confirmation of DMA ${ }^{\mathrm{III}}$ synthesized in the laboratory 118

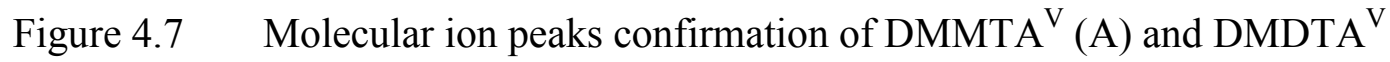
(B)

Figure 4.8 Experimental and calculated Raman spectra of $\operatorname{DMMTA}^{\mathrm{V}}(\mathrm{A})$ and $\operatorname{DMDTA}^{\mathrm{V}}(\mathrm{B})$

Figure 4.9 Mass spectrum fragmentations of DMA ${ }^{\mathrm{III}}$ Cys by ESI-MS 124

Figure 4.10 Experimental and calculated Raman spectra of $\mathrm{DMA}^{\mathrm{III}} \mathrm{GS}(\mathrm{A})$ and $\mathrm{DMA}^{\mathrm{III}}$ Cys (B) 125 


\section{ABBREVIATIONS}

AFM

$\mathrm{AgNF}$

AgNPs

AgNPs-C

AgNPs-Citrate

AgNPs-S

AgNPs-Spermine

APTMS

As

As ${ }^{\text {III }}$

$\mathrm{As}^{\mathrm{V}}$

B3LYP

CE

CTAB

DFT

DI water

$\mathrm{DMA}^{\mathrm{III}}$

$\mathrm{DMA}^{\mathrm{III}} \mathrm{Cys}$

$\mathrm{DMA}^{\mathrm{III}} \mathrm{GS}$

$\mathrm{DMA}^{\mathrm{V}}$

DMA $^{\mathrm{V}}-$ Salt

DMDTA $^{\mathrm{V}}$

DMMTA $^{\text {V }}$
Atomic force microscopy

Silver nanofilm

Silver nanoparticles

Citrate coating silver nanoparticles

Citrate coating silver nanoparticles

Spermine coating silver nanoparticles

Spermine coating silver nanoparticles

3-Aminopropyl)trimethoxysilane

Arsenic

Arsenite

Arsenate

Becke, 3-parameter, Lee-Yang-Parr

Capillary electrophoresis

Cetrimonium bromide

Density functional theory

Deionized water

Dimethylarsinous acid

S-(Dimethylarsenic) cysteine

Dimethylarsinous glutathione

Dimethylarsinic acid

Sodium cacodylic

Dimethyldithioarsinic acid

Dimethylmonothioarinic acid 


$\begin{array}{ll}\text { DMMTA }^{\mathrm{V}}(\mathrm{GS}) & \text { Dimethylarsinothioyl glutathione complex } \\ \text { ESI-MS } & \text { Electrospray mass spectrometer } \\ \text { EXAFS } & \text { X-ray absorption fine structure } \\ \text { FT-ICR-MS } & \text { Fourier transform ion cyclotron resonance mass spectrometry } \\ \text { HPLC } & \text { High performance liquid chromatography } \\ \text { IC } & \text { Ion chromatography } \\ \text { ICP-MS } & \text { Inductively coupled plasma mass spectrometer } \\ \text { LDA } & \text { Linear discriminant analysis } \\ \text { LOD } & \text { Limit of detection } \\ \text { MMA } & \text { Monomethylarsonous acid } \\ \text { MMA } & \text { Monomethylarsonic acid } \\ \text { MMMTA } & \text { Monomethylmonothioarsonic acid } \\ \text { PCA } & \text { Principle components analysis } \\ \text { RMS } & \text { Root mean square error } \\ \text { ROS } & \text { Reactive oxygen species } \\ \text { SDD } & \text { Stuttgart/Dresden double- } \zeta \\ \text { SDS } & \text { Sodium dodecyl sulfate } \\ \text { SERS } & \text { Surface-enhanced Raman spectroscopy } \\ \text { Sodium-DMDTA } & \text { Sodium dimethyldithioarsinate } \\ \text { TOF-MS } & \text { Time-of-flight mass spectrometer } \\ \text { XANES } & \text { X-ray absorption near edge structure } \\ & \end{array}$




\section{Chapter 1}

Introduction, Problem Statement, Objectives and Hypotheses 


\subsection{Relationship between arsenic and human health}

\subsubsection{Arsenic source and distribution}

Arsenic can be introduced into the environment through both natural and anthropogenic sources. Arsenic is the $20^{\text {th }}$ most abundant element in crustal rock, with an average concentration of $2-3 \mathrm{mg} / \mathrm{kg}$. Inorganic arsenic compounds are the major naturally existing arsenicals, including arsenite $\left(\mathrm{As}^{\mathrm{III}}\right)$ and arsenate $\left(\mathrm{As}^{\mathrm{V}}\right)$. Arseniccontaining minerals were widely found in the rocks presenting as orpiment $\left(\mathrm{As}_{2} \mathrm{~S}_{3}\right)$, realgar (AsS), arsenopyrite (FeAsS), arsenolite $\left(\mathrm{As}_{2} \mathrm{O}_{3}\right)$, olivenite $\left(\mathrm{Cu}_{2} \mathrm{OHAsO}_{4}\right)$, cobaltite $(\mathrm{CoAsS})$ and proustite $\left(\mathrm{Ag}_{3} \mathrm{AsS}_{3}\right)[1,2]$. Two significant natural environmental processes, including weathering ractions and volcanic emmissions, are responsible for the universal introduction and distribution of arsenicals into ecosystem cycling (e.g., groundwater, sediment, soil etc $[3,4])$. Globally, naturally existing arsenicals do not pose substantial health hazard and risk towards human beings, because of the generally low arsenic concentration in the environment. However, in some particular areas of world, geological arsenic sources have caused severe public health issues. In Bangladesh and West Bengal, alluvial Ganges aquifers used for public water supply are polluted with naturally occurring arsenic. This has endangered the health of millions of people; arsenic concentrations up to $1000 \mu \mathrm{g} / \mathrm{l}$ have been detected [5]. In Taiwan a condition known as blackfoot disease is common due to well drinking water contamined by arsenic. In the southwest regions of this island, high arsenic concentrations have been found in groundwater originating from deep artesian wells in sediments which include fine sands, muds and black shale [4]. 
Human activities introduce an extreme amount of arsenic into the ecosystem in modern society. Mining [6, 7], application of pesticides and herbicides in agriculture [8, 9], burning of fossil fuels [10], and the poultry industry [11] contribute major arsenic contamination to air, water and soil. Arsenic is released into the environment primarily via mining, smelting and ore beneficiation processes, since arsenic is a natural component of lead, zinc, copper and gold-bearing ores [12]. Coal burning usually does not pose any health risks towards human beings, because the arsenic content in coals is less than $5 \mathrm{mg}$ $\mathrm{kg}^{-1}$ [13]. However certain sources of coal contain an extremely high concentration of arsenic (up to $9600 \mathrm{mg} \mathrm{kg}^{-1}$ ). This material can cause serious arsenic pollution issues if used improperly. For example, when power plants have to use coal containing a high concentration of arsenic in the developing countries, polluted water and air are common [14]. Arsenic-containing pesticides were been widely used by American farmers before 1900 , including lead arsenate, calcium arsenate. During this time there was no regulation for the usage of arsenic pesticides, and they were applied widely in the U.S. agriculture. White arsenic was extensively used for agricultural purposes in the US with 10,800 tons of arsenic-containing insecticides consumed in 1995 [15]. During the early 2000s, organic arsenicals were being added to animal feeds [16]. Recently the US FDA rescinded approval for three arsenic drugs, which have been used as food additives for chickens, turkeys and pigs to prevent disease, increase feed efficiency and promote growth [17]. The use of arsenic in the preservation of timber has also led to contamination of the environment. Chromated copper arsenate (CCA) is a wood preservative that protects wood products from the decaying effects of fungi and insects [18]. Large quantities of these timbers have been used in outdoor playground facilities. 
With a long-term exposure to outdoor weathering conditions, arsenic compounds present in the wood will leach out [19], and has great potential hazard towards human, especially children who play in the playground because young children have a high frequency of hand-to-mouth behavior which is a major pathway for arsenic ingestion [18].

\subsubsection{Arsenic human exposure pathways}

Drinking water is a significant source for human exposure to arsenic in many areas in the world, because drinking water is consumed on a daily base. There are about 30 million of people in Bangladesh, 6 million in West Bengal, India and over 1 million in Vietnam suffering from the poisoning due to arsenic contaminated groundwater [20]. The majority of arsenic species found in groundwater are mainly inorganic arsenic forms, including arsenite $\left(\mathrm{As}^{\mathrm{III}}\right)$ and arsenate $\left(\mathrm{As}^{\mathrm{V}}\right)[21,22]$.

Arsenic exposure from food sources has attracted much attention in the past decade. Since humans are at the top of the food chain, human exposure to arsenic depends on the surrounding environment. Many food contamination events may be attributed to the arsenic, including, surface water and soil contaminated by arsenic, using arsenic polluted water to irrigate crops, organic arsenicals used in food additives in poultry industry, seafood dietary preference, etc. Major sources of arsenic contaminated food include rice and rice related food products $[23,24]$ and seafood $[25,26]$. Because of daily consumption, these food products can pose a chronic health hazard to the human body. Usually, rice grain contamination by arsenic occurs in areas close to mining fields [27] or due to irrigation with contaminated underground water [28]. Contaminated seafood often results from the food chain bioaccumulation [25, 29]. 
Inhalation of arsenic contaminated soil, dust and air can be a major pathway for arsenic exposure for some groups in the population. Workers in the mining industry, smelting industry and silicon factory tend to inhale a large quantity of inorganic arsenic during their long-term working history [30-33]. Depending on the workplace, the blood arsenic speciation and concentration varies. Workers in the glass industry and arsenic manufacturing tend to have the highest concentration of arsenic in their blood [32].

Arsenic occupational exposure in copper factories has been reported to have a positive relationship with the lung cancer cases. Furthermore, it was also revealed that smoking has a significant contribution to arsenic exposure [34]. Burning coal with a high concentration of arsenic could cause the exposure through coal fly ash [35]. Arsenicals have been used as medication/poison for a very long time. For example, arsenic trioxide obtained from smelting copper was used as a drug and as a poison as early as $2000 \mathrm{BC}$; Fowler's solution ( $1 \%$ potassium arsenite, $\left.\mathrm{KAsO}_{2}\right)$ was used as a tonic for anaemia and rheumatism in the 1800s [36] and arsenic trioxide was used to treat granulocytic leukemia in China [37].

\subsubsection{Arsenic uptake in human body}

Arsenicals are introduced into the human body via ingestion and inhalation, with subsequent uptake by the gastrointestinal tract and lung. Once adsorbed by human gastrointestinal tract and lung, it is eventually metabolized in certain human cells, such as liver cells $[38,39]$. The uptake of toxic inorganic arsenicals, such as arsenite and arsenate, is well studied. These two arsenicals have significant difference in protonation ability in the cellular matrix. Therefore, they have different mechanisms of transportation into cells. Arsenite has a very high pKa value and thus it is usually neutralized in the cellular matrix, 
on the other hand, arsenate has a very low pKa value and it is negatively-charged in the cellular matrix. It is believed that arsenite transfers into cells via diffusion [38, 40], because there is a linear relationship between arsenite cellular level and the arsenite concentration in the extracellular matrix, This relationship was not affected by a membrane sulfhydryl modifiers or energy poisons $[38,40]$. The energy poisons stops cellular energy supply and disrupts carrier-mediated uptake systems. The arsenate uptake is inhibited by high concentration of phosphate in the cells, which indicates that the uptake of arsenate is assisted by a phosphate carrier protein [40].

\subsubsection{Arsenic induced diseases/cancers}

Arsenic has been recognized as a group 1 carcinogen to humans by the International Agency for Research on Cancer. The adverse health effects of arsenic strongly depend on dose, duration of exposure, and the nutrition status of the exposed population. Generally, human exposure to arsenic can be categorized into two processes, chronic exposure and acute/lethal exposure. Taking drinking water as an example, in long-term chronic arsenic exposure, the arsenic concentration range may range from $136 \sim 1000 \mu \mathrm{g} / \mathrm{l}$, which is significantly higher than the minimum recommended concentration of $10 \mathrm{ppb}$ proposed by the US Environmental Protection Agency. A lethal dose for human beings was estimated to be $1 \mathrm{mg} / \mathrm{kg} /$ day [41], and it is less than $100 \mathrm{mg}$ for a adulate weigh less than $200 \mathrm{lb}$. While another study claimed the acute poisoning ranges from $100 \mathrm{mg}$ to $300 \mathrm{mg}$ [42]. Exposures to arsenic at a low dose over a long term can cause multisystem diseases, including cancer. Because of the high affinity between arsenic and keratin, arsenic accumulates in scleroproteins such as the hair and nails [43]. The study of arsenic speciation in hair/nails has been employed as an effective biomarker 
of arsenic intake in exposed populations [44, 45]. The initial symptom of arsenic poisoning is hyperpigmentation palmar and solar keratosis, induced by the deposition of arsenic on the skin [42]. With arsenic exposure over a long term, the human body can suffer from a number of diseases, including respiratory/pulmonary system diseases, cardiovascular failure, the hematological problems including anemia, leucopenia, and thrombocytopenia, and hepatic system damage, causing cancer [46]. It is well known that chronic arsenic exposure of highs concentration in drinking water has been associated with a series of cardiovascular diseases, including hypertension, ischaemic heart disease and carotidatherosclerosis. Epidemiological studies also have shown that chronic arsenic exposure is associated with increased morbidity and mortality from cardiovascular disease $[47,48]$. The occurrence of diabetes mellitus was also found to be associated with the inorganic arsenic exposure. For example, a survey of 163 subjects having keratosis revealed that diabetes was prevalent among those subjects in western Bangladesh [49]. In United States, urine arsenic determination in a cross-sectional study of 788 adults indicated that type 2 diabetes has a positive correlation with the total urinal arsenic concentration. In this study it was found that chronic, low to moderate concentrations of arsenic may cause type 2 diabetes [50]. Acute arsenic exposure can cause early symptoms such as abdominal pain, dysphagia, nausea, vomiting, diarrhea, headache, restlessness to patients. As the poisoning progresses, patients may develop gastrointestinal irritation and bleeding, which can lead to massive fluid and electrolyte losses potentially causing the the death of patients $[51,52]$. For the treatment of acute arsenic poisoning, dimercaprol has been used used to chelate arsenic in the body fluids [51, 52]. 


\subsection{Cellular stress from arsenic metabolism in human}

\subsubsection{Cellular redox system}

Cells are the fundamental units of human beings; the health status of cells is directly related to human health. A human cell consists of many organelles, including the nucleus, Golgi body, ribosomes, mitochondria, and cytoplasm. These are enclosed by a cellular membrane. The mitochondria plays a imperative role in cells, involving the oxidation of glucose from the cytoplasm to produce adenosine triphosphate (ATP), which is the carrier of energy for most cellular metabolism. During the mitochondrial oxidization of organic compounds, a superoxide anion is generated by electron leakage [53], which induces the generation of other reactive oxygen species (ROS), including hydroxyl, peroxyl and alkoxyl radicals. Reactive oxygen species play important roles in the regulation of cell survival [53]. In healthy cells, the redox balance is controlled through generation and elimination of ROS/RNS in the physiologic environment. Usually, the generation of ROS happens within the mitochondria in cells [54], and cells have an antioxidant defense system to eliminate ROS stress. This includes the following enzymatic scavengers such s superoxide dismutase (SOD), catalase, and glutathione peroxidase [55]. Glutathione is the substrate of glutathione peroxidase which is active during the elimination of ROS. The concentration of glutathione in the cellular environment is usually maintained at millimolar concentration levels [56]. Thus it is obvious that glutathione is critical for the proper concentration of ROS maintenance. 


\subsubsection{Arsenic inhibits glycolytic energy metabolism}

The human body requires energy to meet its metabolic requirements, and glucose is one of the major energy supplies for human beings. In human cells, the glycolytic reaction breaks down glucose step by step and energy is generated during these processes as ATP is formed in the cells. A glucose molecule chelates with a phosphate molecule at the beginning reaction of the glucose oxidization in the cells. Owing to the structural similarity between arsenate and phosphate, the presence of arsenate will greatly affect the cellular glycolytic reaction by substituting phosphate in the oxidation of glucose molecules [57]. Free phosphate ions are constantly required during glycolysis for the generation of ATP; arsenic disturbs this biological reaction by complexation with ADP into ADP-As, the reactant of the ATP. However, ADP-As is easier to hydrolyze when compared to ATP, a disturbance occurs in cellular processes.

\subsubsection{Arsenic deplete cellular glutathione}

Inorganic arsenic has a very high affinity towards thiol-containing small molecules in the cytoplasm, including glutathione, cysteine, and proteins. Once arsenic enters the human bloodstream and is delivered to cells, it binds to thoil-containing proteins, especially enzymes, and will depress enzymatic activities leading to cell malfunction.It can form chelating complexes with glutathione and cysteine, which are the antioxidants in the cellular plasma, thus imposing oxidative stress on targeted cells. Under chronic arsenic exposure, the cellular glutathione will be depleted and thus cause oxidative damage to various cell components. Studies of patients suffering from chronic arsenic exposure indicate that long-term exposure causes significant oxidative stress on 
cells, including protein oxidation, cellular membrane damage, and an increase in lipid peroxides in blood serum $[58,59]$.

\subsubsection{Arsenic suppress cellular enzymatic activities}

The normal functioning of cells depends on enzymatic activities in the cellular plasma. Enzymes play a critical role in cells, being the catalysts that increase the rate of all biochemical reactions within cells [60]. Arsenic toxicity is believed to be related to the binding of arsenite to enzyme thiols, because of its high affinity towards these compounds. Consequentially, inorganic arsenic can induce enzymes malfunction once it enters the cell. Pyruvate dehydrogenase (PDH), a mitochondrial multi enzyme complex containing many sulfhydryl groups, catalyzes the oxidative decarboxylation of the end product of glycolysis. It has been demonstrated that the presence of arsenic trioxide can cause the inactivation of PDH, due to the generation of ROS [61]. Inorganic arsenic also inhibits deoxyribonucleic acid (DNA) repair process, however, a detailed mechanism is not yet available [62].

Arsenic metabolism causes DNA damage and disturbs DNA repair. Certain types of damage to DNA result in structural changes that create issues when the DNA is replicated [63]. Damage to DNA frequently happens in cells and can occur due to ROS generation during cellular metabolism. In order to maintain accurate genetic information, cells have enzymatic mechanisms to repair broken DNA sequences, involving a collection of processes by which DNA damages are identified and later corrected. As mentioned in the previous section, arsenic metabolism will escalate the ROS concentration in the cellular environment and can worsen DNA damage [64-66]. As a consequence of the high affinity of arsenic towards thiol-containing biomolecules, 
arsenic can inhibit enzymes during DNA repair, such as the bacterial Fpg protein and the mammalian XPA protein [67]. In addition direct damage can occur through DNA nicking by MMA ${ }^{\mathrm{III}}$ and $\mathrm{DMA}^{\mathrm{III}}[68-70]$.

\subsection{Current analytical techniques for arsenic speciation}

\subsubsection{Chromatography-based methods}

Arsenic speciation in biological matrices has been routinely conducted using techniques coupling chromatography/electrophoresis separations and sensitive detectors. The separation methods that have been explored in arsenic speciation include high performance liquid chromatography (HPLC) [71-74], capillary electrophoresis (CE) [7577], and ion chromatography (IC) [78, 79]. Among these separation techniques, HPLC is the most popular technique used in the arsenic speciation because of its ability to perform separations in a wide range of research areas, using various sample matrixes. Depending on the need to emphasize analytical sensitivity or structure determination of the arsenic species, one could select different detection methods, such as inductively coupled plasma mass spectrometer (ICP-MS) [80], electrospray mass spectrometry (ESI-MS) [72], or time-of-flight mass spectrometry (TOF-MS) [81]. Sample preparation in all these speciation methods involves the release of arsenicals from the biological matrix, followed by clean-up of the sample matrix. Extraction using an organic solvent or strong acid/base has the potential to change the integrity of many arsenic species. For instance, MMA ${ }^{\mathrm{III}}$ or DMA $^{\mathrm{III}}$, two important arsenic metabolites, are unstable and readily oxidized during extraction [5]. Arsenate could be reduced to arsenite and form $\mathrm{As}(\mathrm{GS})_{3}$ in the cellular environment because of the presence of high concentrations of glutathione [56]. The $\mathrm{As}(\mathrm{GS})_{3}$ complex is not stable in the mice bile and it is only stable in the presence of 5 
$\mathrm{mM}$ of glutathione. It is understandable that $\mathrm{As}(\mathrm{GS})_{3}$ could be decomposed and oxidized during the solvent extraction procedure. Additionally, recently observed thiolated arsenicals, such as dimethylmonothioarsinic acid $\left(\mathrm{DMMTA}^{\mathrm{V}}\right)$, dimethyldithioarsinic acid $\left(\right.$ DMDTA $\left.^{\mathrm{V}}\right)$ and trivalent arsenicals conjugate with thiol-containing molecules in biological samples, and are sensitive to acidic/basic chemical environment and temperature changes [82]. It has become clear that problems in sample preparation can cause misleading results, consequently hindering the arsenic speciation studies in biological matrices. Therefore, it is necessary to develop an arsenic speciation method in biological matrices that can remain as much as possible the integrity of arsenic species or to eliminate extraction steps during the sample preparation and analysis completely. Beside potential degradation of the sample in chromatographic studies, identical retention during chromatographic separation could also undermine our understanding of arsenic metabolism. For example, recently dimethylarsinothioic acid was mistakenly determined in wool extract as DMA ${ }^{\mathrm{III}}$ [83], which brought lots of research attention towards thiolcontaining arsenicals and some sulfur-involved metabolic pathways were proposed[84, 85]. The case of wrong / inconclusive results owing to overlapped HPLC retention times has not only happened in arsenic speciation, but also has occurred in certain protein identification studies [86, 87].

\subsubsection{X-ray absorption spectroscopy related methods}

The technique of X-ray absorption spectroscopy (XAS) has also been employed in arsenic speciation, including X-ray absorption near edge structure (XANES) and extended X-ray absorption fine structure (EXAFS). The XANES method involves the near edge absorption structure which is the K-absorption edge (K-edge), which is defined 
as the energy required to remove an electron in the innermost orbit and eject it into the continuum [88], while EXAFS deals with a relatively higher range of energy absorption. Both near edge structure and fine structure are determined by the following factors, interatomic distances, chemical valence, local coordination environmental, coordination number, and oxidation state [89-93]. Both techniques were employed for arsenic speciation in environmental samples, including arsenic species in samples collected from mining field [94] and arsenic in plants (e.g., rice samples) [95, 96]. Application of these techniques has also been used for arsenic speciation in in-situ environments [97, 98]. Bacquart et al. [98] employed a micro-XANES to study the arsenic speciation inside of frozen hydrated cells. The successful application of these methods for arsenic speciation in many environmental and biological samples provides opportunities that the chromatography-based techniques cannot offer, because X-ray based methods are nondestructive to the sample and also simplify sample preparation. However the satisfactory application of these techniques is highly dependent on the availability of arsenic standards. As described by Foster et al. [94], data analysis was accomplished using the EXAFSPAK programs. In this study, the XANES spectra of model compounds and samples were background subtracted and normalized to the edge jump, and then the second derivative of each XANES spectrum was then calculated using a $2 \mathrm{eV}$ smoothing interval. Another data processing method employed in XANES involved a linear combination fitting of the spectra, in which the XANES spectra of the samples were fitted to these standards to further calculate the relative percentage of each species. In these studies, the application of XAS was mostly restricted to environmental samples, and samples in solid form were required during sample measurement. Although the non- 
destructive sample preparation would be promising for its application in arsenic speciation in a biological matrix, the application in the biological matrix is rarely reported, which could be the result of a requirement for solid or powder samples. Also, the availability of the synchrotron radiation X-ray absorption is limited, generating a real problem for routine analytical needs

\subsection{Surface-enhanced Raman spectroscopy for arsenic detection}

\subsubsection{Surface-enhanced Raman spectroscopy for bioanalytical application}

In comparison with other analytical techniques for analysis of biological samples, SERS is a rapid and non-destructive photon scattering technique, which provides fingerprint information regarding molecular vibrational energy levels [99]. With a significant enhancement in the Raman signal via the interaction between the incident light and the well-designed metallic surface of a substrate, SERS has emerged as a novel technique for the analysis of a broad range of chemical substances in complex biological matrices. Compared to infrared spectroscopy, Raman spectroscopy has minimal interference from water molecules, which exists in most biological samples. Thus this unique advantage allows Raman spectra to be obtained directly from biological matrices [100]. Different strategies for SERS studies in biological matrices have been found in the current literature. Firstly, biofluids, including saliva, urine, tear, blood, blood serum, were directly placed on SERS-active substrates or mixed with metallic colloidal suspensions [101-108]. The SERS signals were generated owing to the evaporation of solution evaporation or the creation of hot spots from the aggregation of nanoparticles caused by introducing aggregation agents. Overall, SERS signals obtained using this method was not of high quality, and the possible reason for poor signal response could be the 
following: the SERS-active substrates were not optimized in the size and material and the aggregation process is difficult to control. Secondly, statistical methods like the chemometrics was employed for biofluids Raman spectra data analysis. Through employing principal components analysis (PCA) and linear discriminant analysis (LDA) in most cases, this methodology was successfully applied for cancer detection and disease diagnosis with a high percentage of diagnosis accuracy [109-115]. Chemometrics might not be applicable in arsenical's speciation study due to a limited number of diagnostic Raman vibrational bands. Thirdly, SERS metallic substrates of well-organized surface morphology were employed for biomolecules measurement, such as nanofilm fabricated by a self-assembled membrane, closely packed nanorods [116-119]. The well-organized substrate surface ensured strong SERS signals upon the sample introduction. Fourthly, the methodology of immunoassay, taking advantage of the binding reaction between antigens and antibodies, was carried out by using SERS tags and surface modification of antibodies [120-123]. Usually, magnetic materials were capsulated into nanoparticles for further magnetic separation nanoparticles from matrices as soon as the equilibrium is established between the interactions between nanoparticles with biological matrices using this method. Furthermore, derivatization of targeted molecules was employed when the molecule's Raman activity was poor. Taking the glucose determination for example [124, 125], boric acid was employed in order to capture glucose in the sample matrix. Very few molecules have a universal binding reaction or specific derivatization reaction with arsenicals, thus the application of SERS tags and derivatization methods may be limited. 


\subsubsection{Surface-enhanced Raman spectroscopy for arsenic detection}

The very first application of SERS in arsenic analysis can be dated back to 1988, when Greaves and Griffith obtained arsenate SERS signal response around $780 \mathrm{~cm}^{-1}$ by directly mixing arsenate with silver nanoparticles [126]. In the past several decades, many studies have been carried out for the applications of SERS in arsenic analysis and speciation with an emphasis on improvement of the substrates for strong SERS signal production. Metallic colloidal suspensions [127-130] and metallic nanofilms [131-138] are two common substrates for SERS measurement while some indirect SERS methods employing nanoparticles for arsenite analysis $[127,129,130]$ has also been reported. Upon the introduction of arsenite into modified nanoparticle solutions, these aptamers could release from the nanoparticles and bind with arsenite. The release of aptamers on the nanoparticles causes a significant drop in the surface charge and thus the nanoparticles aggregate and Raman report molecules' signals are greatly enhanced. As a consequence of its simplicity, metallic colloidal suspensions as SERS substrates are most widely used. Modification in the shape of the nanoparticles has often been performed in order to enhance the SERS signal, structures such as nanostar, nanorods, etc have been used [139-141]. Metallic nanofilm is the other popular SERS substrate for arsenic speciation, and it is easy to obtain arsenic SERS signals from nanofilms because of evaporation of droplets or diffusion of arsenicals on the nanofilm. Currently, different strategies are employed for nanofilm fabrication to study arsenic species, such as the modified mirror reaction for silver nanofilm deposition [137, 138, 142], coffee-ring evaporation [131], through the Langmuir-Blodgett assembly [132], electrothermal deposition $[134,135]$. Overall the surface morphology of the nanofilm is critical for the 
sensitivity and reproducibility of arsenic analysis. Until now, SERS application for arsenic analysis has been mainly limited to inorganic arsenic species, such as $\mathrm{As}^{\mathrm{III}}$ or $\mathrm{As}{ }^{\mathrm{V}}$ and environmental samples, such as well water or underground water. Comparing to inorganic arsenic compounds, analysis of organic arsenicals using SERS was much less reported [134, 135, 143]. Furthermore, the SERS application of arsenic speciation in biological matrices has not been explored to the best of our knowledge. Performing SERS for arsenic speciation in biological media is challenging because the presence of complicated electrolytes and biomolecules. Previous studies have shown that nanoparticles aggregate immediately upon mixing with a physiological buffer solution $[144,145]$. The uncontrolled aggregation of nanoparticles can hinder the SERS signal response in the physiological matrix. Furthermore, nanoparticles can be coated with a film of biological molecules in some biological matrices, such as proteins in blood and cellular extracts. This is known as a protein corona and has been studied in drug delivery systems using nanomaterials [146]. However, coating nanoparticle surfaces with proteins can reduce the SERS active area on the surface and thus quench the SERS signal. Therefore, minimal coating of biomolecules on nanoparticles surface with particle aggregation is necessary for successful SERS applications in cellular matrices.

\subsubsection{Current issues in surface-enhanced Raman spectroscopy for arsenic detection}

The development of SERS application in arsenic speciation is still in its infancy, and currently reports are mostly focused on environmental samples $[126,128,132,133$, 136-138, 142, 147-149]. Studies of arsenic SERS responses in the presence of cellular matrices or physiologic samples have scarcely been explored. Arsenic speciation studies in cellular matrices are more important than the determination of total arsenic 
concentration, as these studies will yield the arsenical bioavailability and provide necessary information for toxicological evaluation. Furthermore, most previous reports were focused on inorganic arsenicals, such as $\mathrm{As}^{\mathrm{III}}$ and $\mathrm{As}^{\mathrm{V}}$. Arsenic metabolism in cellular environments will convert inorganic arsenicals into various organoarsenicals. However, Raman spectra of many potential arsenical metabolites were not available in the literature or never studied, therefore, it is urgent to obtain this firsthand SERS information prior to arsenic speciation for cellular or physiological samples.

Current arsenic speciation methods may not be capable of depicting correct arsenical profiles in cellular matrices, since chromatographic-based methods require sample preparation using strong acids or oxidative reagents, which may cause changes in the integrity of arsenic species. XAS-based methods may be able to preserve the arsenic species in the sample, however, the ideal sample state is solid, and cellular samples are rarely reported in the XAS studies. Here, SERS is proposed for arsenic speciation in the cellular matrix, because it has the following advantages high sensitivity because of the great signal enhancement from the interaction of arsenicals with nano size metallic surface and non-destructive sample preparation.

There are two SERS substrates employed mostly in current reports, metallic colloidal suspension and metallic nanofilms. Both of these have high potential in the SERS application of arsenic speciation. My dissertation studies are mostly related to employment of these two SERS substrates to explore their potential in arsenic speciation.

\subsection{Problem Statement}

The application of SERS in arsenic speciation is still at the early research stage. Currently, arsenic speciation using SERS is mainly restricted to inorganic arsenic species, 
such as $\mathrm{As}^{\mathrm{III}}$ and $\mathrm{As}^{\mathrm{V}}$. Two common existing organarsenicals, e.g., monomethylarsonic acid $\left(\mathrm{MMA}^{\mathrm{V}}\right)$ and dimethylarsinic acid $\left(\mathrm{DMA}^{\mathrm{V}}\right)$ found in human body were not studied by SERS. Furthermore, arsenic speciation studies were mostly conducted for environmental samples, while arsenic speciation in biological or physiological samples was rarely reported in the literature. It is unknown if SERS is capable of simultaneous detection of arsenic species in the presence of biological matrices.

Application of SERS in biological matrices holds promise because it is a nondestructive analytical approach and it could have high sensitivity detection capabilities because of the great enhancement factor which originates from surface plasmon resonance (SPR) on the nanosize metal surface. However, to the best of our knowledge, Raman spectra of some important arsenic metabolites have been rarely recorded and reported. These important arsenic metabolites are mostly unstable, including the following, monomethylarsonous acid $\left(\mathrm{MMA}^{\mathrm{III}}\right)$, and dimethylarsinous acid $\left(\mathrm{DMA}^{\mathrm{III}}\right)$, dimethylmonothioarinic acid $\left(\right.$ DMMTA $\left.^{\mathrm{V}}\right)$, dimethyldithioarsinic acid $\left(\right.$ DMDTA $\left.^{\mathrm{V}}\right)$, S(Dimethylarsenic) cysteine (DMA $\left.{ }^{\mathrm{III}}(\mathrm{Cys})\right)$ and dimethylarsinous glutathione (DMA $\left.{ }^{\mathrm{III}} \mathrm{GS}\right)$. It is therefore important to characterize these arsenicals' Raman spectra or fingerprint Raman frequencies prior to applying SERS analysis of arsenic speciation under the influence of biological matrices. The availability of Raman spectra is the prerequisite requirement for SERS studies and the Raman spectra will be helpful for the assignment of SERS spectra.

\subsection{Hypotheses}

The established objectives were guided by the following hypotheses: 
1. Electrostatic interactions between arsenicals and the nanoparticle surface in the colloidal suspension are critical for an optimal SERS response. To be more specific, the adsorption of arsenic compounds onto the nanoparticles surface in the aqueous solution will mostly be controlled by the electrostatic interactions. These adsorptive effects will determine whether SERS signals are generated or not. Selected arsenicals have different molecular structures and they should have specific fingerprint signal responses, which will makes the simultaneous detection possible. Different molecular surface coatings of these nanoparticles will be used in order to obtain nanoparticles with variations in surface charge, permitting the impact of surface charge to be monitored.

2. Compared to hot spots generated by the aggregation of gold/silver nanoparticles in the solution, hot spots on nanofilm is relatively easy to control, since nanofilms are usually consisted of closely parking nanoparticles on solid surface. The surface morphology is relatively controllable to obtain an organized surface with nanoparticles during the fabrication procedure. This closely parking nanofilm should be able to yield strong SERS signal for arsenic compounds SERS simultaneous detection.

3. Density functional theory (DFT) is capable of predicting molecular properties, including vibrational profiles. The discrepancy between calculated and experimental Raman frequencies of a standard molecule will determine whether the DFT calculation is reliable or not and comparison methods will be adapted from previous work [162-165]. Different empirical scaling factors will be employed to reduce the difference. After optimization of this method, the DFT calculation will be employed to generate theoretical Raman spectra of unknown arsenicals and detect specific vibrational modes of 
arsenicals. Using this data, the experimental Raman spectra of unknown arsenicals can be identified by a combination of calculated and experimental Raman spectra.

\subsection{Objectives}

The present work involves a study of the applications of SERS in arsenic speciation and the identification of Raman spectra of major potential arsenic metabolites.

1. Employing silver nanoparticles as the SERS substrate to explore the possibility of arsenic speciation.

Silver nanoparticles of different surface charges will be used to study arsenic speciation under the influence of a cellular matrix. The response of individual arsenic species will be obtained in nanoparticles of different surface charge first and then arsenic speciation in the presence of a cellular matrix will be carried out using the same nanoparticles. To have a better understanding of the differences in SERS signal response, adsorption of arsenicals on the silver nanoparticles colloidal suspensions and arsenicals in the cellular matrice will be studied. Size and charge effects of nanoparticles in the cellular matrice will be monitored to obtain an in-depth understanding of the SERS signal response.

2. Study of arsenic speciation employing silver nanofilm

Arsenic speciation of four common existing arsenicals will be carried out on the silver nanofilm (AgNF). Different approaches will be carried out to fabrication nanofilm with relatively organized surface morphology, including a modified mirror reaction [142] and a self-assembled metal colloid monolayers method [166]. Individual arsenic fingerprint signals will be obtained first to see whether these signals are different from signals in the colloidal suspensions. Furthermore, arsenic speciation uses nanofilm will 
be studied. The mixture will be prepared in different solution with different $\mathrm{pH}$ to modulate the deprotonation states of these arsenicals. If this approach is not able to fulfill the purpose of the simultaneous detection, the coffee ring effect will be employed. Arsenic SERS signals from the center to the edge of the dry coffee ring will be measured.

3. Identification of sulfur-containing arsenicals' Raman spectra

Raman spectra of potential arsenical metabolites will be studied to assign and identify arsenicals' fingerprint Raman frequencies. The combination of theoretical calculation with experimental studies will be carried out in order to determine the fingerprint Raman vibrations of different arsenicals, including, MMA ${ }^{\mathrm{III}}$, DMA ${ }^{\mathrm{III}}$, DMDTA $^{\mathrm{V}}$, DMMTA ${ }^{\mathrm{V}}$, DMA ${ }^{\mathrm{III}} \mathrm{Cys}$ and DMA ${ }^{\mathrm{III}} \mathrm{GS}$. Most of these arsenicals will be synthesized in the laboratory and their molecular structures will be identified with a molecular mass spectrometer. Density functional theory will be employed for theoretical Raman spectra simulation in order to assign experimental Raman spectra. 


\section{Chapter 2}

Potential Application of SERS for Arsenic Speciation in Biological Matrices

Part of this chapter was accepted for publication in the journal of Analytical and Bioanalytical Chemistry. Adapted with permission from Yang, M., Matulis, S., Boise, L. H., McGoron, A. J., \& Cai, Y. (2017). Potential application of SERS for arsenic speciation in biological matrices. Analytical and Bioanalytical Chemistry. doi:10.1007/s00216-017-0434-3. Copyright (2017) Springer and Copyright Clearance Center. 


\subsection{Abstract}

Speciation of arsenic is usually carried out using chromatography-based methods coupled with spectroscopic determination; however, the inevitable procedures involving sample preparation and separation could potentially alter the integrity of the arsenic metabolites present in biological samples. Surface-enhanced Raman spectroscopy (SERS) could be a promising alternative for providing a reliable arsenic analysis under the influence of a cellular matrix. A method for arsenic speciation using SERS in cellular matrix was developed in this study and four arsenicals were selected, including arsenite $\left(\mathrm{As}{ }^{\mathrm{III}}\right)$, arsenate $\left(\mathrm{As}^{\mathrm{V}}\right)$, monomethylarsonic acid $\left(\mathrm{MMA}^{\mathrm{V}}\right)$ and dimethylarsinic acid $\left(\mathrm{DMA}^{\mathrm{V}}\right)$. Silver nanoparticles in the form of colliodal suspension with different surface charges, i.e. coated with citrate (AgNPs-Citrate) and spermine (AgNPs-Spermine) were employed as SERS substrates. Adsorption of arsenicals on nanoparticles in colloidal suspensions and the cellular matrix along with the $\mathrm{pH}$, size and zeta potential of the colloidal suspensions were investigated for better understanding of SERS signals response of arsenicals in the colloidal suspensions or under the influence of cellular matrix. Arsenicals showed substantially different SERS responses in the two colloidal suspensions, mainly due to the distinct difference in the interaction between the arsenicals and the nanoparticles. Arsenic speciation in cell lysate could be successfully carried out in AgNPs-Spermine suspension, while AgNPs-Citrate could not yield significant SERS signals under the experimental conditions. This study proved that AgNPs-Spermine colloidal suspension could be a promising SERS substrate for studying arsenic metabolism in a biological matrix, reducing the bias caused by traditional techniques that involve sample extraction and pretreatment. 


\section{Keywords: Arsenic ・SERS・Speciation • Nanoparticles ・ Cell lysate}

\subsection{Introduction}

Millions of people around the world are suffering from arsenic-induced diseases, including various types of cancers [132, 167-169]. Generally, human beings are exposed to arsenicals via ingestion and inhalation. The metabolism of arsenicals in the human body involves a multistep transformation in the arsenic species, including reduction/oxidation, methylation, and thiolation reactions [170, 171], ending up with methylated arsenic compounds such as monomethylarsonic acid $\left(\mathrm{MMA}^{\mathrm{V}}\right)$ and dimethylarsinic acid $\left(\mathrm{DMA}^{\mathrm{V}}\right)$ in urine and blood. Methylation of inorganic arsenic is traditionally considered to be a detoxification process for harmful inorganic arsenicals, since the toxicity of $\mathrm{MMA}^{\mathrm{V}}$ or $\mathrm{DMA}^{\mathrm{V}}$ is much lower than that of inorganic arsenicals [84, 172]. However, this view has been challenged because of the studies on monomethylarsonous acid $\left(\mathrm{MMA}^{\mathrm{III}}\right)$ and dimethylarsinous acid $\left(\mathrm{DMA}^{\mathrm{III}}\right)$, two recently found organic arsenicals from urine samples of patients chronically exposed to inorganic arsenicals in drinking water $[173,174] . \mathrm{MMA}^{\mathrm{III}}$ and $\mathrm{DMA}^{\mathrm{III}}$ are possible metabolic products of arsenic in humans and their toxicity was found to be higher than the inorganic $\mathrm{As}^{\mathrm{III}}[175,176]$. The toxicity of arsenic is greatly related to its chemical forms, hence a full investigation of arsenic speciation in biological samples, such as cell, tissue and blood, becomes critical for a better understanding of arsenic toxicity and mode of action.

Arsenic speciation in biological matrices has been routinely conducted using techniques coupling chromatographic/electrophoretic separation and sensitive detectors. The separation methods that have been explored in arsenic speciation include high performance liquid chromatography (HPLC) [33, 72, 73, 177], capillary electrophoresis 
(CE) [75-77], and ion chromatography (IC) [78, 79]. Based on either the analytical sensitivity needed or the molecular information of the arsenic species desired, one could select different detection methods, such as inductively coupled plasma mass spectrometer (ICP-MS) [80], electrospray mass spectrometer (ESI-MS) [72], and time-of-flight mass spectrometer (TOF-MS) [81]. Sample preparation in all these speciation methods is an essential step for successful detection and involves the release of arsenicals from the biological matrix and the cleanup of the matrix. Extraction using organic solvents or strong acid/base has a great potential to alter the integrity of many arsenic species. For instance, $\mathrm{MMA}^{\mathrm{III}}$ or DMA ${ }^{\mathrm{III}}$, two important arsenic metabolites are unstable and readily oxidized during the course of extraction [178]. Arsenate could be reduced to arsenite and form $\mathrm{As}(\mathrm{GS})_{3}$ in the cellular environment due to the presence of a high concentration of glutathione (GSH) [56]. As(GS) $)_{3}$ could be decomposed and oxidized during the solvent extraction process since the $\mathrm{As}(\mathrm{GS})_{3}$ complex is only stable in the present of certain levels of GSH. Additionally, the recently observed thiolated arsenicals, such as dimethylmonothioarsinic acid $\left(\mathrm{DMMTA}^{\mathrm{V}}\right)$ and dimethyldithioarsinic acid $\left(\mathrm{DMDTA}^{\mathrm{V}}\right)$ or trivalent arsenicals conjugated with thiol-containing molecules in biological samples, are only stable under a narrow range of $\mathrm{pH}$ and are sensitive to temperature changes [82]. It becomes clear that sample preparation can cause misleading results, consequently hindering the study of arsenic speciation in biological matrices. Therefore, it is necessary to develop an arsenic speciation method in biological matrices that can retain as much as possible the integrity of the arsenic species profile, or ideally to eliminate the extraction steps during sample preparation and analysis completely. 
Surface-enhanced Raman spectroscopy (SERS) is a promising technique that can be potentially used in arsenic speciation in biological samples. SERS is a rapid and nondestructive photon scattering technique, providing fingerprint information regarding molecular vibrational energy levels [99]. SERS has emerged as an important technique for the analysis of a broad range of chemical substances in complex biological matrices. For instance, metallic nanoparticles were employed to determine some chemical substances present in various biological samples including saliva, urine, tear, blood, blood serum, by directly introducing nanoparticles into these biological matrices [101108, 179]. However, SERS signals are usually not strong enough or suffer from interference from components in biological matrices. In some cases, derivatization was employed to enhance the SERS response if the Raman cross section of the molecule of interest was poor $[124,125]$. A useful derivatization reaction or binding agent has not been reported for the arsenic species with biological interest, thus limiting the application of SERS in arsenic speciation. In other cases, complicated SERS signals are interpreted using the methods of chemometrics, such as principal components analysis and linear discriminant analysis. This method was successfully applied for cancer detection and the diagnosis of other diseases with a fairly high accuracy [109-115]. However, since principal component analysis (PCA) and linear discriminant analysis (LDA) usually need a lot of vibrational frequencies to perform the data analysis, this approach is not suitable for studying arsenic owing to the limited number of Raman vibrational bands.

The very first application of SERS for arsenic analysis can be dated back to 1988 when Greaves and Griffith obtained an arsenate SERS signal response around $780 \mathrm{~cm}^{-1}$ by directly mixing arsenate with silver nanoparticles [126]. In the past several decades, 
SERS has been applied in arsenic analysis and speciation in water with an emphasis on improvement of the substrates for strong SERS signal production. Metallic colloidal suspension [127-130] and metallic nanofilm [131-138] are two common substrates used for SERS measurement while some indirect SERS methods employing nanoparticles for arsenite analysis $[127,129,130]$ have also been reported. Due to simplicity, metallic colloidal suspensions are most widely used as SERS substrates. Modification of the shape of the nanoparticles, such as nanostar and nanorods, has often been performed in order to enhance the SERS signal [139-141]. Another popular SERS substrate is metallic nanofilm since SERS signals can be readily obtained due to the evaporation of the droplet sample which brings the analytes close to the nanofilm surface. Up to now, the application of SERS for arsenic analysis has been mainly limited to inorganic arsenic species, such as $\mathrm{As}^{\mathrm{III}}$ or $\mathrm{As}^{\mathrm{V}}$ in water samples, such as from wells or other underground water sources $[126,128,132,133,136-138,147]$. Comparing to inorganic arsenic compounds, the analysis of organic arsenicals using SERS has been much less reported $[134,135,143]$. Furthermore, the study of arsenic speciation in biological matrices using SERS has not been explored to the best of our knowledge. Performing SERS for arsenic speciation in biological media is challenging because of the presence of complicated electrolytes and biomolecules $[144,145]$. The uncontrolled aggregation of nanoparticles could hinder SERS signal response in a physiological matrix. Nanoparticles could become coated with a film of biological molecules in some biological matrices, such as proteins present in blood and cellular extract. This phenomenon is named protein corona and has been studied in drug delivery using nanomaterials [146]. The nanoparticle surface coated with proteins could reduce the SERS active area on its surface and thus 
quench the SERS signal. Therefore, lessening the coating of biomolecules on the nanoparticle's surface and uncontrolled aggregation is necessary for the successful application of SERS on arsenic speciation in biological matrices. In addition, SERS technique as a promising analytical tool for detection of inorganic arsenic species in water media is well discussed recently [180].

In this study, a method for arsenic speciation using SERS, based on silver colloidal suspensions in the presence of cellular matrix, was explored. As the first step for our research on arsenic speciation in biological samples using SERS, we decided to provide a proof-of-concept study focusing mainly on the fundamentals that determine the SERS measurement of selected arsenicals. We studied the electrostatic interactions between arsenicals and nanoparticles in colloidal suspension and the controlling factors, alterations of the nanoparticle surface through coating, and interferences of cell lysates to arsenic measurement by SERS. Four arsenic species that are widely studied in biological matrices, namely $\mathrm{As}^{\mathrm{III}}, \mathrm{As}^{\mathrm{V}}, \mathrm{MMA}^{\mathrm{V}}$ and $\mathrm{DMA}^{\mathrm{V}}$ were included in this study. To the best of our knowledge, this is the first study attempting to perform SERS arsenic speciation under the influence of a biological matrix. The $8226 / \mathrm{S}$ multiple myeloma cell line was selected to provide a cell lysate and arsenicals were added to the cell lysate for the simulation of cellular environment. Special attention was given to controlling and improving the electrostatic interactions between particles and arsenic in the colloidal suspension as it is critical for successful SERS measurements in solution. Two silver nanoparticles (AgNPs) with different surface charge were employed. The size, zeta potential and $\mathrm{pH}$ of AgNPs colloidal suspensions under the influence of cell lysate were 
monitored to understand the impact of the cellular matrix on the arsenic SERS signal response.

\subsection{Material and Methods}

\subsubsection{Material and Chemicals}

Sodium metaarsenite, $98 \%\left(\mathrm{As}^{\mathrm{III}}\right)$, sodium arsenate dibasic, $99 \%\left(\mathrm{As}^{\mathrm{V}}\right)$, cacodylic acid sodium salt, 98\% $\left(\mathrm{DMA}^{\mathrm{V}}\right)$ were purchased from Sigma-Aldrich, USA. Monosodium acid methane arsonate sesquihydrate, 99.5\% $\left(\mathrm{MMA}^{\mathrm{V}}\right)$ was purchased from Chem Service, USA. Silver nitrate (99.99\%) was purchased from STREM Chemicals, USA. Spermine tetrahydrochloride (99\%, powder) was purchased from Alfa Aesar, USA. Sodium citrate dehydrate (Granular certified), $\mathrm{NaOH}, \mathrm{HCl}, \mathrm{MgCl}_{2}, \mathrm{Na}_{2} \mathrm{SO}_{4}, \mathrm{NaCl}, \mathrm{KNO}_{3}$ were purchased from Fisher Scientific Inc as certified A.C.S grade. The phosphate-buffered saline (PBS), $1 \times$ PBS was prepared in the laboratory. All solutions were prepared in deionized water (DI water, 18.2 $\mathrm{M} \Omega$, Barnstead Nanopure Diamond).

The selected cell line was 8226/S multiple myeloma, which was purchased from the American Type Culture collection (ATCC, Manassas, VA), maintained at $37{ }^{\circ} \mathrm{C}$ in a humidified atmosphere with 5\% $\mathrm{CO}_{2}$ in RPMI-1640 media, supplemented with $100 \mathrm{U}$ $\mathrm{ml}^{-1}$ of penicillin, $100 \mu \mathrm{g} \mathrm{ml}^{-1}$ of streptomycin, $10 \%$ heat inactivated fetal bovine serum, and $2 \mathrm{mM}$ L-glutamine (all culture reagents from Cellgro, MediaTech, Herndon, VA). The cells were cultured at a concentration of $2 \times 10^{5}$ cells $/ \mathrm{ml}$ and then harvested by centrifugation at $1000 \mathrm{rpm}$ for 5 minutes, washed with PBS once, and spun down again, and then the pellets were frozen in liquid nitrogen and stored at $-20^{\circ} \mathrm{C}$ for future usage. 


\subsubsection{Instrumentation}

An Elan DRC-e (Perkin-Elmer) ICP-MS was employed for arsenic analysis. The ICP-MS was equipped with an auto sampler, a cyclonic spray chamber, and a Meinhard nebulizer. All arsenic samples were diluted in $2 \%$ nitric acid and spiked with 20 ppb yttrium prior to ICP-MS quantification analysis. The following signals were monitored: 75 for arsenic, 89 for yttrium (as internal standard). A standard calibration curve was obtained on a daily base.

The Raman spectrometer used was a Perkin-Elmer RamanStation 400F Raman spectroscopy, employing a $785 \mathrm{~nm}$ diode laser with average power of $100 \mathrm{~mW}$ at the sample and 100-micron spot size. This Raman station was equipped with a $\mathrm{x}-\mathrm{y}-\mathrm{z}$ sample stage accepting standard 96-well plate with a glass bottom. The 96-well plates were purchased from PerkinElmer (Waltham, MA) and were reused by cleaning with cotton swabs and drying under a stream of nitrogen gas. Raman spectra were acquired by transferring $200 \mu \mathrm{l}$ of solution into wells of the 96-well plate. Typical sample measurement was carried out for 1 second of exposure and 4 times of accumulation for each measurement. This Perkin-Elmer Ramanstation was also equipped with a PerkinElmer RamanMicro 300 system, which had three optical objectives $5 \times, 20 \times$ and $50 \times$. Usually, the $20 \times$ optical objective was used for better sample focus and lower background signal. For SERS measurements using an optical objective, $40 \mu 1$ of solution was dropped onto a glass microscope slide and SERS signals were obtained under the same conditions as using 96 well plates.

The size and zeta potential of nanoparticles were measured with a Malvern Zetasizer Nano-ZS (Westborough, MA). The hydrodynamic diameters reported in this 
study represent the average particle diameter "z-average" intensity peak as a function of size. UV-vis absorption spectra of silver nanoparticles were obtained using a Cary 300 UV-vis spectrometer. The $\mathrm{pH}$ measurements were normally carried out on a Fisher Scientific Accumet Research AR15 $\mathrm{pH} / \mathrm{mV} /{ }^{\circ} \mathrm{C}$ Meter, while for $\mathrm{pH}$ measurement of low volume solutions ( $<50 \mu 1), \mathrm{pH}$ testing paper with multiple $\mathrm{pH}$ ranges was used.

A Pall Microsep centrifugal device (centrifuge filters) (PALL Corporation, USA) with $1 \mathrm{~K} \mathrm{Da}$ molecular weight cutoff was employed for the centrifugal filtration experiments. This centrifugal device consisted of a sample reservoir and a filtrate receiver separated by a semi-permeable membrane (Omega membrane), which has low protein-binding. The filtration was carried out at room temperature using a Marathon 21000R benchtop centrifuge (Fisher Scientific, USA) for 25 minutes at a speed of 8000 rpm (over $5000 \mathrm{~g})$.

\subsection{Experimental Procedures}

2.4.1 Synthesis and characterization of silver nanoparticles

AgNPs of different surface coating were synthesized in the laboratory. The procedures for synthesizing citrate coated silver nanoparticles (AgNPs-Citrate) were adopted from Lee \& Meisel [181] with a minor modification. Briefly, all glassware were cleaned by emerging in Aqua Regia solution $\left(\mathrm{HCl} / \mathrm{HNO}_{3}=3: 1\right.$, v/v) overnight and rinsing with a large amount of tap water and DI water and finally dried in an oven at 80 ${ }^{\circ} \mathrm{C}$ prior to use. Fifty $\mathrm{ml}$ of $1 \times 10^{-3} \mathrm{~mol} / \mathrm{l}$ of $\mathrm{AgNO}_{3}$ was added to a $250 \mathrm{ml}$ round bottom flask and then heated to boil in an oil bath under vigorous stirring with a condenser equipped to reflux. Sodium citrate dihydrate $(1 \%, 2 \mathrm{ml})$ was introduced drop by drop and then kept heating for one hour. Characteristics of the AgNPs-Citrate, such as 
hydrodynamic size, zeta potential (surface charge), $\mathrm{pH}$ and UV-vis absorption were recorded soon after the colloids were synthesized.

The synthesis of spermine coated silver nanoparticles (AgNPs-Spermine) was performed following Faulds \& Graham [182] with a minor modification. Prior to reaction, the glass flask was filled with $30 \mathrm{ml}$ of $0.001 \mathrm{~mol} / 1$ spermine stirring for 30 minutes to form a positively charged glass surface to prevent the adhering of positively charged nanoparticles, and then rinsed with a large amount of DI water multiple times and dried under nitrogen gas. The glass flask was wrapped with aluminum foil to avoid exposure to light. In brief, $20 \mathrm{ml}$ of $1 \times 10^{-3} \mathrm{~mol} / 1$ of $\mathrm{AgNO}_{3}$ was mixed with $10 \mu \mathrm{o}$ of $0.1 \mathrm{~mol} / 1$ spermine in a round bottom flask and then degassed with a flow of nitrogen for 30 minutes under vigorous stirring. Fresh prepared $\mathrm{NaBH}_{4}(0.1 \mathrm{~mol} / \mathrm{l}, 50 \mu \mathrm{l})$ was then added and kept stirring for one hour. Upon introduction of $\mathrm{NaBH}_{4}$, the solution turned yellow in seconds and the solution was stored in the refrigerator overnight prior to use in order to let excess $\mathrm{NaBH}_{4}$ degrade. Characteristics of AgNPs-Spermine were obtained in the same way as AgNPs-Citrate.

2.4.2 SERS measurement of arsenic species in different silver nanoparticle suspensions AgNPs-Citrate or AgNPs-Spermine colloidal suspension (480 $\mu$ l) was mixed with $20 \mu 1$ of $100 \mathrm{ppm}$ arsenic standards individually. Different amounts of $\mathrm{Na}_{2} \mathrm{SO}_{4}$ solutions $(0.5 \mathrm{M})$ were introduced to initiate the aggregation of AgNPs-Citrate and the solution was vortexed for 30 seconds, while no salt-initiated aggregation was carried out for the AgNPs-Spermine suspension. Within 3 minutes, $200 \mu 1$ of the resulted AgNPs colloidal suspension was transferred to the 96-well plate. Raman signals were obtained as described in the Instrumental section. 
Experiments assessing the adsorption of arsenic species on nanoparticles were conducted to provide a better understanding of arsenic SERS signal response from different AgNPs colloidal suspensions. Adsorption experiments were carried out by adding $60 \mu \mathrm{l}$ of a $1000 \mathrm{ppm}$ individual arsenic standard into $1440 \mu \mathrm{l}$ of an AgNPs colloidal suspension in a $1.5 \mathrm{ml}$ centrifuge tube. The centrifuge tube was shaken for one hour at a speed of $80 \mathrm{rpm}$ on an orbital shaker. After shaking, the solution was split into two parts, one of which was aggregated by introducing $30 \mu 1$ of $0.5 \mathrm{~mol} / 1 \mathrm{Na}_{2} \mathrm{SO}_{4}$ and the other was used as control (no $\mathrm{Na}_{2} \mathrm{SO}_{4}$ added). Afterward, both solutions were centrifuged at $8000 \mathrm{rpm}$ (over 5000g) for 10 minutes and the supernatants were analyzed for arsenic using ICP-MS. To calculate the mass balance during adsorption, experiments were also carried out by substituting the AgNPs solution with deionized water. All experiments were performed in triplicate. Results of arsenic concentrations in the supernatant were expressed as the mean \pm standard deviation $(\mathrm{SD})(\mathrm{n}=3)$. Statistical analysis was performed and one-way ANOVA was employed with a $P<0.05$ being considered to be significant.

\subsubsection{Interactions between arsenic and cellular matrix}

Interactions between arsenicals and biomolecules in the cell lysate, through binding/adsorption, during the SERS measurement of arsenic were inevitable. Thiolcontaining molecules present in the cell lysate could complex directly with trivalent arsenicals. Arsenicals might also interact with proteins in the cell lysate via electrostatic forces. Centrifugal filtration was used to study the adsorption of arsenic in the cellular matrix. The centrifugal filtration was able to let small arsenic compounds/complexes pass through the molecular cutoff membrane $(1 \mathrm{~K} \mathrm{Da})$ and to retain arsenicals binding to 
proteins or biomolecules. A pellet of $2 \times 10^{5}$ cells was thawed and $900 \mu 1$ DI water was added. A sonication probe (Fisher Scientific Sonic Dismembrator Model 100, Waltham, MA) was employed to break up the cells following a previously reported procedure [183]. The cell lysate $(45 \mu \mathrm{l})$ was mixed with $25 \mu 1$ of $100 \mathrm{ppm}$ individual arsenic standard solution and then $430 \mu 1$ of $1 \times$ PBS buffer were added. Control experiments were carried out by substituting the cell lysate with an equal volume of DI water in order to study the distribution of arsenic in the sample reservoir and the filtrate receiver of the centrifugal device. The PBS buffer and cell lysate were analyzed separately for arsenic background. All solutions were incubated at $37^{\circ} \mathrm{C}$ for 30 minutes and transferred into the centrifugal device equipped with a membrane of molecular weight cut-off of $1 \mathrm{~K} \mathrm{Da}$. Centrifugation was carried out at $8000 \mathrm{rpm}$ (over $5000 \mathrm{~g}$ ) for 25 minutes. The volume of solutions in the sample reservoir (upper) and the filtrate receiver (lower) were recorded approximately according to the weight assuming the density of the solution is equal to water. Arsenic concentrations in the sample reservoir and the filtrate receiver were measured by ICP-MS. All adsorption experiments were performed in triplicate.

\subsubsection{Effect of cell media on SERS measurement of arsenic species}

The cell lysate solution $\left(2 \times 10^{5}\right.$ cells in $900 \mu$ of DI water) was diluted with DI water ten times in sequence in order to prepare cell lysate in $900 \mu 1$ solutions with different concentrations of $2 \times 10^{4}, 2 \times 10^{3}$, and $2 \times 10^{2}$ cells, respectively. Individual arsenic standard solutions (100 ppm, $2 \mu 1$ of each standard) were added to $90 \mu 1$ of the diluted cell lysate solutions, followed by adding $2 \mu \mathrm{l}$ of DI water. This procedure generated diluted lysate solutions containing $2 \mathrm{ppm}$ of each arsenic species. These solutions were vortexed for 30 seconds and then placed on an orbital shaker at $80 \mathrm{rpm}$ for 30 minutes 
prior to use. For measurement of arsenic using SERS, $90 \mu 1$ of AgNPs was mixed with 10 $\mu 1$ of the diluted cell lysate containing $2 \mathrm{ppm}$ of arsenic. For AgNPs-Citrate, $5 \mu 1$ of 0.5 $\mathrm{mol} / 1 \mathrm{Na}_{2} \mathrm{SO}_{4}$ was introduced in order to aggregate the nanoparticles. Finally, $40 \mu 1$ of the mixed solution was transferred onto a glass slide and SERS signals were obtained by using the $20 \times$ optical objective.

In order to better understand the SERS signal from arsenic under the influence of cell lysate, the colloidal suspensions properties including, particles size, surface charge and $\mathrm{pH}$ value were measured upon interaction with different concentrations of cell lysate. The AgNPs colloidal solution containing $2 \times 10^{4}$ cell $/ \mathrm{ml}$ was prepared by adding $90 \mu 1$ of $2 \times 10^{5}$ cell lysate solution (prepared in $900 \mu 1$ DI water) into $910 \mu 1$ of AgNPs colloidal suspension and the solution was vortexed prior to properties measurements. The final concentrations of cell lysate in the AgNPs colloidal suspension were $2 \times 10^{4}, 2 \times 10^{3}, 2 \times 10^{2}$ and $2 \times 10 \mathrm{cell} / \mathrm{ml}$ by using different concentrations of cell lysate solution. The $\mathrm{pH}$ of colloidal suspensions was measured using different $\mathrm{pH}$ test papers because of the small volume of the samples. The size and zeta potential of the AgNPs were measured using the Malvern Zetasizer Nano-ZS. All experiments were carried out in triplicate. Sizes and zeta potentials for all samples were summarized as mean $\pm \mathrm{SD}(\mathrm{n}=3)$ and analyzed using one-way ANOVA and the $P<0.05$ was considered to be significant. 


\subsection{Results and Discussions}

\subsubsection{SERS Measurement of Arsenic Species in Silver Nanoparticle Suspensions}
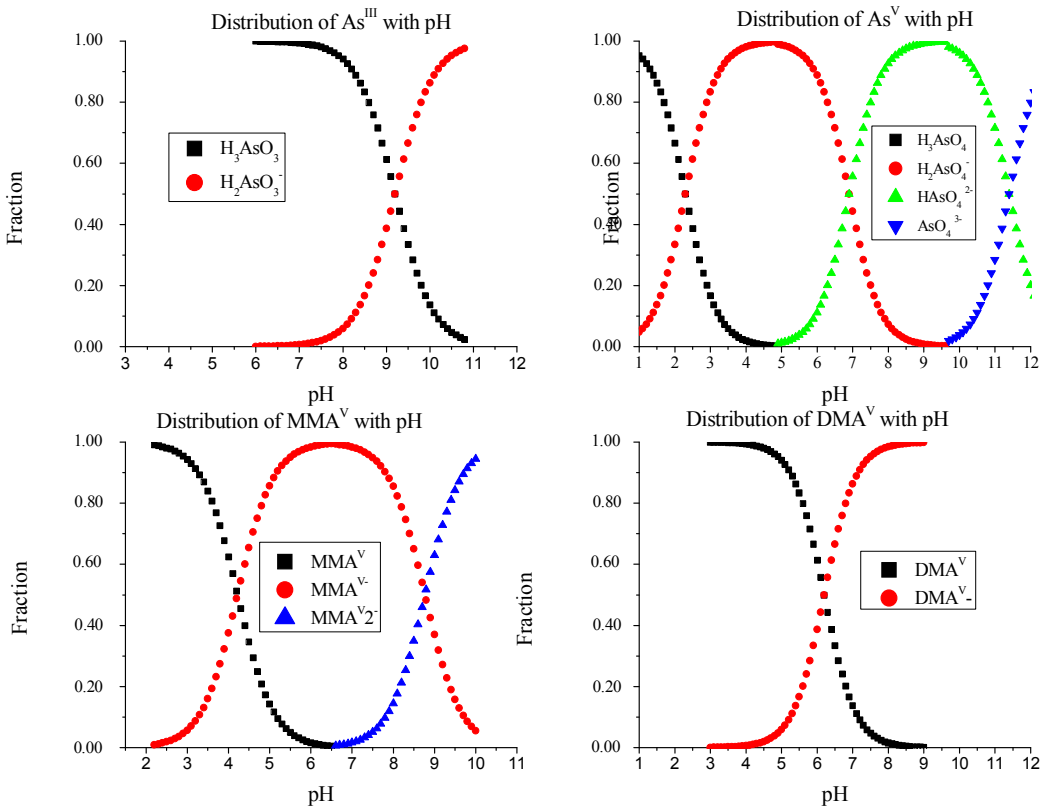

Figure 2.1 The distribution of individual arsenic species in aqueous solution with different $\mathrm{pH}$

The four selected arsenic species have different $\mathrm{pKa}$ values and therefore show different charges at a certain $\mathrm{pH}$ (Fig. 2.1). The characteristics of the synthesized AgNPsCitrate and AgNPs-Spermine are shown in Fig. 2.2. The sizes of the nanoparticles were 60 and $53 \mathrm{~nm}$ for AgNPs-Citrate and AgNPs-Spermine, respectively and the polydispersity index (PDI) values were less than 0.2 , indicating that their hydrodynamic sizes were likely homogeneously distributed in the colloidal suspension. These two AgNPs suspensions had significantly different solution $\mathrm{pH}$, due to the different surface capping agents. AgNPs-Citrate suspension was usually at approximately $\mathrm{pH} 9$ as prepared, while AgNPs-Spermine was about $\mathrm{pH}$ 4. The surface charges of the 
nanoparticles, described in zeta potential, were -50.1 and $+50 \mathrm{mV}$ in AgNPs-Citrate and AgNPs-Spermine suspensions respectively.

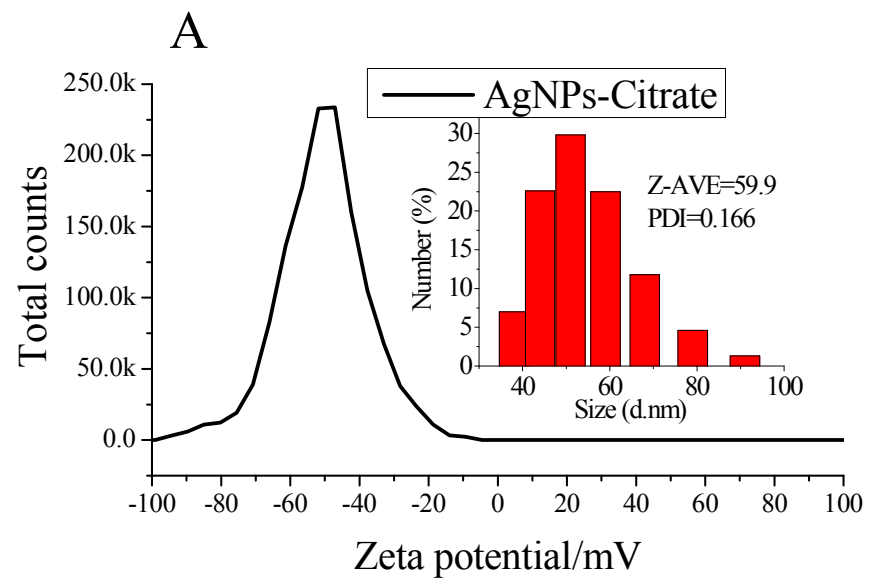

B

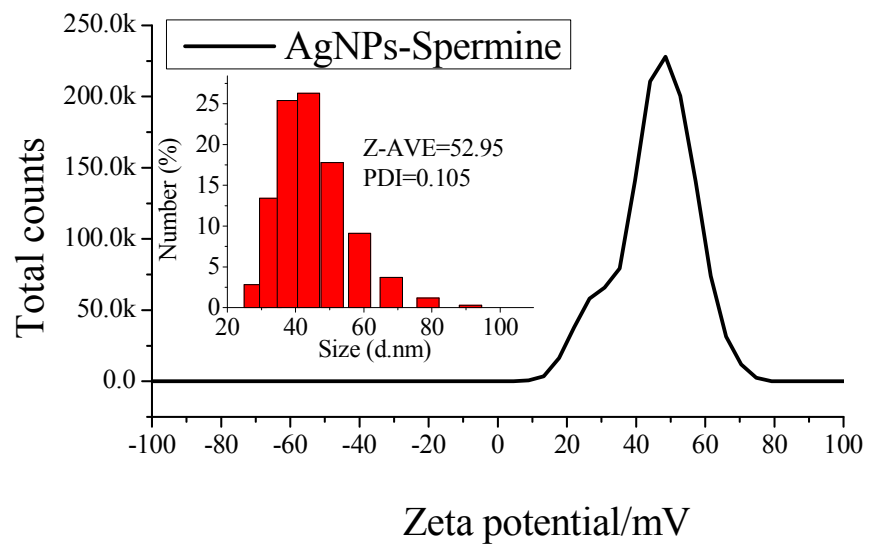

Figure 2.2 Typical zeta potential and size distribution of AgNPs-Citrate (A) and AgNPs-Spermine (B). Both nanoparticles had PDI (polydispersity index) values less than 0.2 , indicating that their hydrodynamic sizes were considered as homogeneous distribution in the colloidal suspension. AgNPs-Citrate suspension was usually at $\mathrm{pH} 9$ as prepared. AgNPs-Spermine suspension was at $\mathrm{pH}$ 4. In AgNPs-Citrate suspension, nanoparticles were coated by negatively charged citrate ions and the zeta potential is negative, while in AgNPs-Spermine suspension, nanoparticles were coated by positively charged spermine ions and the zeta potential is positive. 
Raman spectra of the four arsenicals in AgNPs-Citrate and AgNPs-Spermine colloidal suspension are shown in Fig. 2.3A and B, respectively, and background SERS signals from the colloidal suspensions are in red.
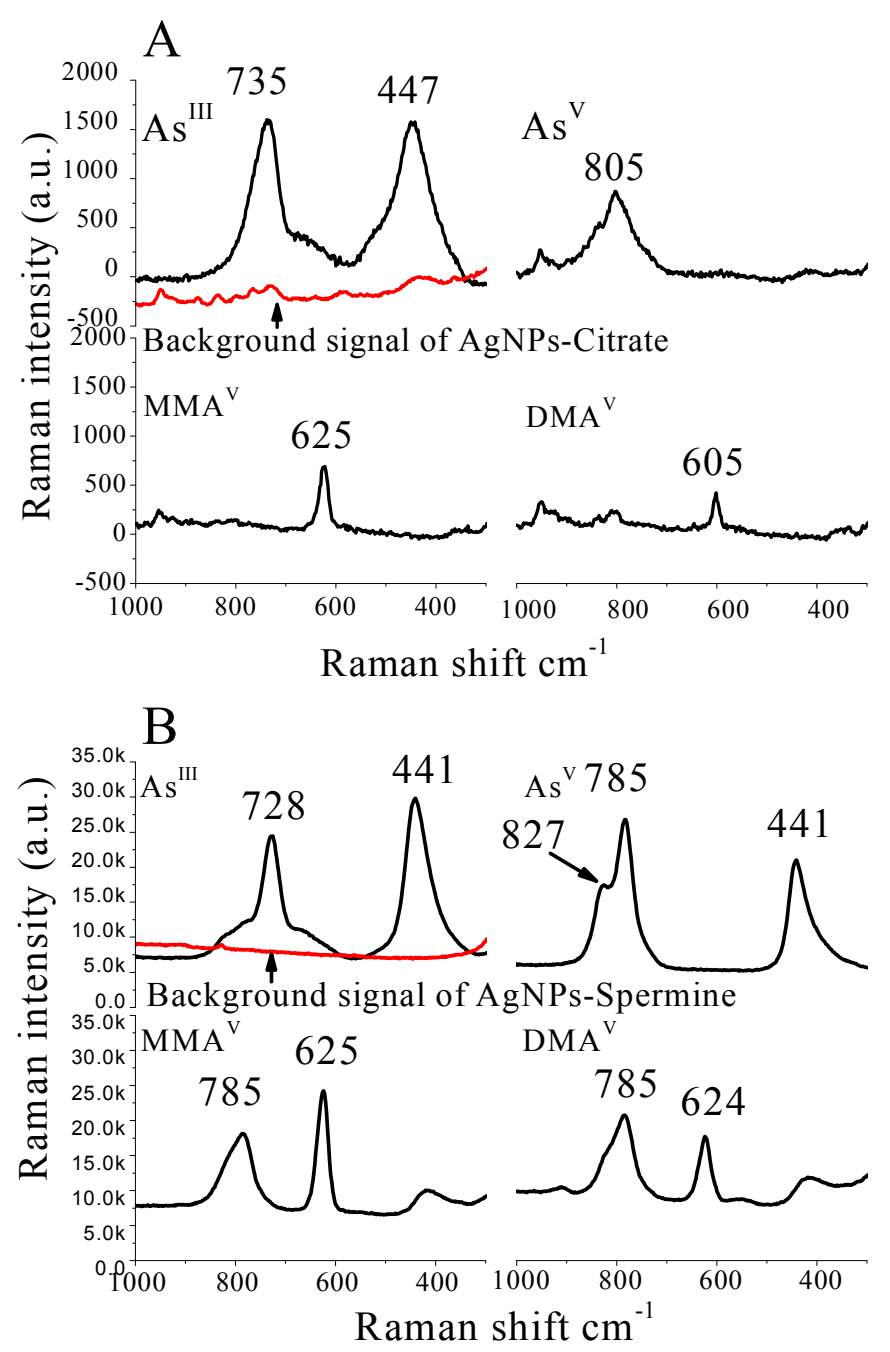

Figure 2.3 SERS spectra of individual arsenic species obtained in colloidal suspensions of AgNPs-Citrate (A) and AgNPs-Spermine (B). Colloidal suspension of 4 ppm of arsenic was prepared by mixing $480 \mu 1$ of AgNPs-Citrate or AgNPs-Spermine with $20 \mu 1$ of $100 \mathrm{ppm}$ individual arsenic solution. The AgNPs-Citrate suspension was then aggregated with $20 \mu \mathrm{l}$ of $0.5 \mathrm{M} \mathrm{Na}_{2} \mathrm{SO}_{4}$, while no such aggregation was performed for AgNPs-Spermine. 
In the AgNPs-Citrate colloidal suspension, there were two characteristic vibrational bands for $\mathrm{As}{ }^{\mathrm{III}}$ with $735 \mathrm{~cm}^{-1}$ resulting from the As-O stretch mode [128, 142, 184]. The peak at $447 \mathrm{~cm}^{-1}$ was not reported previously and could not be assigned in this study. For $\mathrm{As}^{\mathrm{V}}$, one peak at $805 \mathrm{~cm}^{-1}$, corresponding to the $\mathrm{As}-\mathrm{O}$ symmetric vibration $[185,186]$ was observed. For these two organoarsenicals, characteristic As-C stretching vibrations, due to different numbers of methyl group, were identified at 625 and $605 \mathrm{~cm}^{-1}$ as the fingerprint vibration for $\mathrm{MMA}^{\mathrm{V}}$ and $\mathrm{DMA}^{\mathrm{V}}$, respectively [135, 187-189]. The SERS signals of organoarsenicals were much weaker, especially for $\mathrm{DMA}^{\mathrm{V}}$ in comparison to the inorganic arsenic species. For SERS measurement in AgNPs-Spermine suspension, similar results were observed for $\mathrm{As}^{\mathrm{III}}$ with a slight shift in wavenumber for both bands in comparison to that in AgNPs-Citrate, being likely attributed to the difference in chemical environments (Fig. 2.3B). SERS spectra of $\mathrm{As}^{\mathrm{V}}$ showed two vibrational bands, $785 \mathrm{~cm}^{-1}$ being attributed to the As-O symmetric stretch mode [128, 142] (including the shoulder at $827 \mathrm{~cm}^{-1}$, which belonged to the As-O asymmetric stretch mode $[149,189])$ and $441 \mathrm{~cm}^{-1}$ being the superposition stretch of $v_{2}$ and $v_{5}$ of the arsenate molecule $[132,136]$. The two organoarsenicals, $\mathrm{MMA}^{\mathrm{V}}$ and $\mathrm{DMA}^{\mathrm{V}}$ had very similar vibrational profiles with a slight difference in the As-C vibrational frequency. The As-O stretch mode at $785 \mathrm{~cm}^{-1}$ was observed for both compounds and the As-C vibration at $625 \mathrm{~cm}^{-1}$ and $624 \mathrm{~cm}^{-1}$ were found in $\mathrm{MMA}^{\mathrm{V}}$ and $\mathrm{DMA}^{\mathrm{V}}$, respectively. Obviously, clear differences in SERS signal of arsenicals were observed between the two AgNPs colloidal suspensions. Arsenicals had much stronger SERS responses in AgNPsSpermine than in AgNPs-Citrate. The superposition vibration of $\mathrm{As}-\mathrm{O}$ in $\mathrm{As}^{\mathrm{V}}$ was found in AgNPs-Spermine but not in AgNPs-Citrate. The As-O vibration at $785 \mathrm{~cm}^{-1}$ was 
found for both organoarsenicals $[135,187,188]$ in AgNPs-Spermine colloidals suspension, which was based on the fact that both organoarsenicals have the As-O bond and the SERS vibrational frequency of As-O of organoarsenicals was around $780 \mathrm{~cm}^{-1}$ [135], while not in AgNPs-Citrate. Furthermore, the As-C vibrations of the two organoarsenicals at approximately $625 \mathrm{~cm}^{-1}$ were almost the same in AgNPs-Spermine, however, a shift of $20 \mathrm{~cm}^{-1}$ between the two compounds appeared in AgNPs-Citrate (Fig $2.3 \mathrm{~A}$ and $2.3 \mathrm{~B})$.

The electrostatic interactions between arsenicals and nanoparticles in the colloidal suspension plays an important role in arsenicals SERS sensing, making individual arsenicals manifest SERS signals differently in these two colloidal suspensions. Silver nanoparticles employed in this study were coated with different surface capping agents, thus they had different surface charges. Upon introducing arsenic standards, there was no significant $\mathrm{pH}$ change in the AgNPs colloidal suspensions. Arsenicals were in different protonation states in these two suspensions, for instance, $\mathrm{As}^{\mathrm{III}}$ being neutralized $\left(\mathrm{H}_{3} \mathrm{AsO}_{3}\right)$ in the AgNPs-Spermine colloidal suspension, however it was about $50 \%$ negatively charged $\left(\mathrm{H}_{2} \mathrm{AsO}_{3}{ }^{-1}\right)$ and about $50 \%$ neutralized $\left(\mathrm{H}_{3} \mathrm{AsO}_{3}\right)$ in AgNPs-Citrate (Table 2.1).

Table 2.1 Changes in deprotonation of arsenicals in different AgNPs colloidal suspensions

\begin{tabular}{|c|c|c|}
\hline & $\begin{array}{c}\text { AgNPs-Citrate, } \mathrm{pH} 9, \\
\text { negatively charged }\end{array}$ & $\begin{array}{c}\text { AgNPs-Spermine, } \mathrm{pH} \mathrm{4,} \\
\text { positively charged }\end{array}$ \\
\hline $\mathrm{As}^{\mathrm{III}}$ & $50 \% \mathrm{H}_{3} \mathrm{AsO}_{3}, 50 \% \mathrm{H}_{2} \mathrm{AsO}_{3}{ }^{-1}$ & $100 \% \mathrm{H}_{3} \mathrm{AsO}_{3}$ \\
\hline $\mathrm{As}^{\mathrm{V}}$ & $\mathrm{Almost} 100 \% \mathrm{HAsO}_{4}{ }^{-2}$ & Almost $100 \% \mathrm{H}_{2} \mathrm{AsO}_{4}{ }^{-1}$ \\
\hline $\mathrm{MMA}^{\mathrm{V}}$ & $100 \% \mathrm{CH}_{3} \mathrm{As}(\mathrm{OH}) \mathrm{O}_{2}{ }^{-1}$ & $\begin{array}{c}50 \% \mathrm{CH}_{3} \mathrm{As}(\mathrm{OH})_{2}, 50 \% \\
\mathrm{CH}_{3} \mathrm{As}(\mathrm{OH}) \mathrm{O}_{2}^{-1}\end{array}$ \\
\hline $\mathrm{DMA}^{\mathrm{V}}$ & $100 \%\left(\mathrm{CH}_{3}\right)_{2} \mathrm{AsOH}^{-1}$ & $100 \%\left(\mathrm{CH}_{3}\right)_{2} \mathrm{AsOH}_{2}$ \\
\hline
\end{tabular}


The electrostatic attraction between arsenicals and nanoparticles promotes the adsorption of arsenicals onto nanoparticles, thus greatly enhancing the SERS signals. Individual arsenicals have different protonation status in these two colloidal suspensions. For instance, $\mathrm{As}^{\mathrm{V}}$ and $\mathrm{MMA}^{\mathrm{V}}$ were negatively charged ions in both the positively charged AgNPs-Spermine and in the negatively charged AgNPs-Citrate colloidal suspensions. In the AgNps-Spermine suspension, $\mathrm{As}^{\mathrm{V}}$ and $\mathrm{MMA}^{\mathrm{V}}$ are attached onto the nanoparticle's surface via electrostatic interaction, however, in the AgNPs-Citrate suspension, they are repelled by the negatively charged nanoparticle's surface. This could explain why SERS signals in AgNPs-Spermine were stronger than in AgNPs-Citrate. $\mathrm{As}^{\mathrm{III}}$ and $\mathrm{DMA}^{\mathrm{V}}$ were neutralized in AgNPs-Spermine and negatively charged in AgNPsCitrate. $\mathrm{As}^{\mathrm{III}}$ and $\mathrm{DMA}^{\mathrm{V}}$ had poorer SERS signals in AgNPs-Citrate than in AgNPsSpermine, owing to a repulsive interaction between these two arsenicals and the negatively charged AgNPs-Citrate nanoparticles. However, the strong SERS signal for $\mathrm{As}^{\mathrm{III}}$ and $\mathrm{DMA}^{\mathrm{V}}$ in AgNPs-Spermine indicates that these arsenicals were brought to the surface close enough for efficient SERS to occur.

In order to better understand the factors controlling the SERS signals of the four arsenicals in AgNPs-Citrate and AgNPs-Spermine colloidal suspensions, the interactions between arsenicals and nanoparticle were investigated using adsorption experiments (Fig. 2.4). For AgNPs-Citrate (Fig. 2.4A), adding salt (i.e. aggregation of nanoparticles) seems to enhance the adsorption of arsenicals on nanoparticles. This is particularly true for As ${ }^{\mathrm{III}}$. Overall, the adsorption of arsenicals on the AgNPs-Citrate nanoparticles was less than 10\%. A different pattern was observed in AgNPs-Spermine (Fig. 2.4B). The adsorption of $\mathrm{As}^{\mathrm{III}}$ and $\mathrm{As}^{\mathrm{V}}$ on the nanoparticles was about 23 and 15\%, respectively in AgNPs- 
Spermine suspension without addition of salt. Additing salt to the suspension either inhibited the adsorption of inorganic arsenicals or caused the release of adorbed arsenicals from the surface of the nanoparticles. The adsorption of the two organoarsenicals in the AgNPs-Spermine suspension was less than 5\%. The adsorption of arsenicals onto the nanoparticles surface is critical for SERS response because adsorption is an efficient way to bring these arsenicals in the solution close to the surface of AgNPs, ideally with a distance of less than $10 \mathrm{~nm}$ [190]. Adsorption of $\mathrm{As}^{\mathrm{III}}$ and $\mathrm{As}^{\mathrm{V}}$ onto nanoparticles in the AgNPs-Spermine suspension was larger than that in the AgNPsCitrate, being consistent with the fact that stronger SERS signals of $\mathrm{As}^{\mathrm{III}}$ and $\mathrm{As}^{\mathrm{V}}$ in AgNPs-Spermine than in AgNPs-Citrate. However, the adsorption of MMA ${ }^{\mathrm{V}}$ and $\mathrm{DMA}^{\mathrm{V}}$ on the AgNPs-Spermine was poor even though the SERS signals observed for MMA ${ }^{\mathrm{V}}$ and $\mathrm{DMA}^{\mathrm{V}}$ were stronger compared to AgNPs-Citrate, suggesting that other factors, in addition to adsorption of arsenicals on the surface of nanoparticles, contributed significantly to the SERS measurement of organoarsenicals $[135,191]$. 


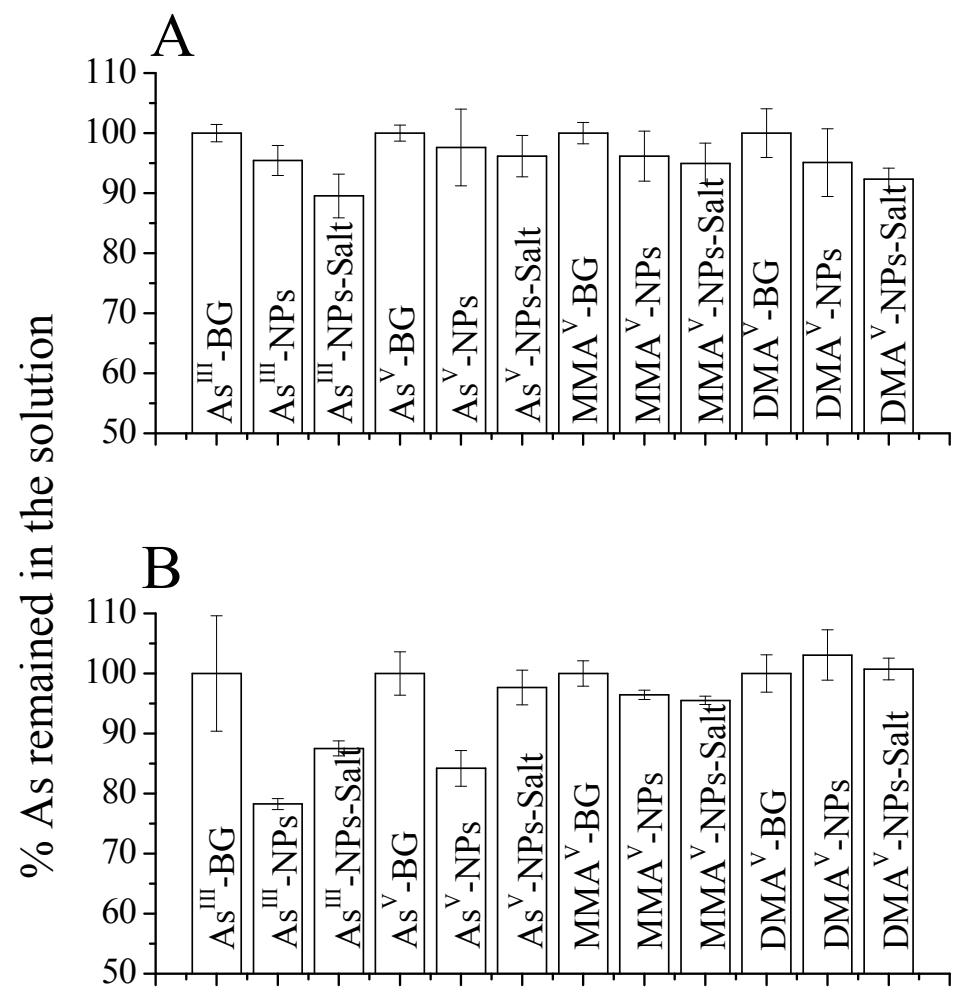

Figure 2.4 Adsorption of arsenic in colloidal suspensions of AgNPs-Citrate (A) and AgNPs-Spermine (B). BG and NPs indicate that the arsenic remained in the solution for controls (prepared in DI water only) and samples with nanoparticle, respectively. Salt represents the results for trials with the addition of nanoparticles and salt. All arsenic concentrations in the BG, NPs and NPs-Salt were significantly different according to One-way ANOVA at $\mathrm{P}<0.05$ level.

Major vibrational frequencies of $\mathrm{As}{ }^{\mathrm{III}}$ and $\mathrm{As}^{\mathrm{V}}$ were reduced 7 and $20 \mathrm{~cm}^{-1}$ when changing from AgNPs-Citrate to AgNPs-Spermine suspensions, respectively, representing a red-shift in wavelength. A possible reason for this difference could be a charge transfer effect $[135,192]$ induced by the stronger adsorption of these two arsenicals onto nanoparticle's surface in the AgNPs-Spermine, as shown in the aforementioned adsorption experiments. Charge transfer excitations could significantly reduce the intrinsic intramolecular excitation of the adsorbate, thus resulting in a red-shift 
[193]. For the two organoarsenicals, SERS signals were feeble in the AgNPs-Citrate suspension, but their characteristic vibrational frequencies of the $\mathrm{As}-\mathrm{C}$ were distinguishable between $\mathrm{MMA}^{\mathrm{V}}\left(625 \mathrm{~cm}^{-1}\right)$ and $\mathrm{DMA}^{\mathrm{V}}\left(605 \mathrm{~cm}^{-1}\right)$. In the AgNPsSpermine suspension, the SERS signals of $\mathrm{MMA}^{\mathrm{V}}$ and $\mathrm{DMA}^{\mathrm{V}}$ were similar to each other. Both organoarsenicals had a $785 \mathrm{~cm}^{-1}$ vibrational band, which was attributed to the symmetric As-O stretch $[187,188]$, and the stretch frequencies of $\mathrm{As}-\mathrm{C}$ in $\mathrm{MMA}^{\mathrm{V}}$ and $\mathrm{DMA}^{\mathrm{V}}$ were 625 and $624 \mathrm{~cm}^{-1}$, respectively. The vibrational band at $785 \mathrm{~cm}^{-1}$ was present in $\mathrm{As}^{\mathrm{V}}, \mathrm{MMA}^{\mathrm{V}}$ and $\mathrm{DMA}^{\mathrm{V}}$. These three pentavalent arsenicals had As-O single bonds in their molecules despite their different deprotonation status in the AgNPsSpermine suspension (Table 3.1). Since there were two methyl groups in $\mathrm{DMA}^{\mathrm{V}}$, the Raman spectra should have two vibrational modes of As-C [187], including symmetric and asymmetric vibrations. The symmetric and asymmetric vibrational frequencies for As $-\mathrm{C}$ in $\mathrm{DMA}^{\mathrm{V}}$ were reported to be different, being 651 and $605 \mathrm{~cm}^{-1}$ respectively [187]. However, there was only one vibrational band found for the As-C vibration in the AgNPs-Spermine for DMA ${ }^{\mathrm{V}}$. This $624 \mathrm{~cm}^{-1}$ vibrational band was not reported previosuly and it might belong to the symmetric vibrational of $\mathrm{As}-\mathrm{C}$ in $\mathrm{DMA}^{\mathrm{V}}[70]$.

\subsubsection{Measurement of Arsenicals using SERS in Cell Lysate}

The potential interactions between arsenicals and cell lysate could inhibit the adsorption of arsenicals onto the nanoparticles, and hence reduce the SERS signal. Experiments were therefore conducted to investigate the interactions of arsenicals and cell lysate using a centrifugal filtration technique. Arsenic concentrations in the sample reservoir and filtrate receiver were monitored individually and arsenic concentration ratios in the sample reservoir vs filtrate receivers are shown in Fig. 2.5. Significant 
differences in arsenic concentrations between the two reservoirs were not observed for both control and samples with cell lysate for all arsenicals, indicating the lack of significant adsorption of arsenicals on biomolecules larger than $1 \mathrm{~K}$ Da. However, the effect of biomolecules less than $1 \mathrm{~K}$ Da could not be evaluated by this experiment.

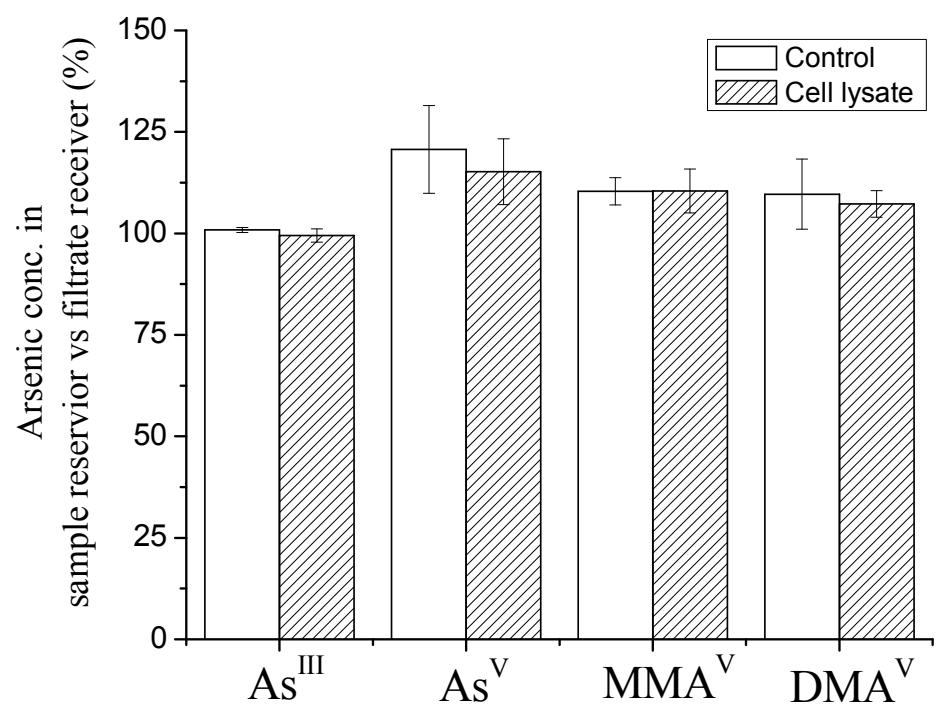

Figure 2.5 Arsenic concentration in the sample reservoir vs. in the filtrate receiver (\%) 

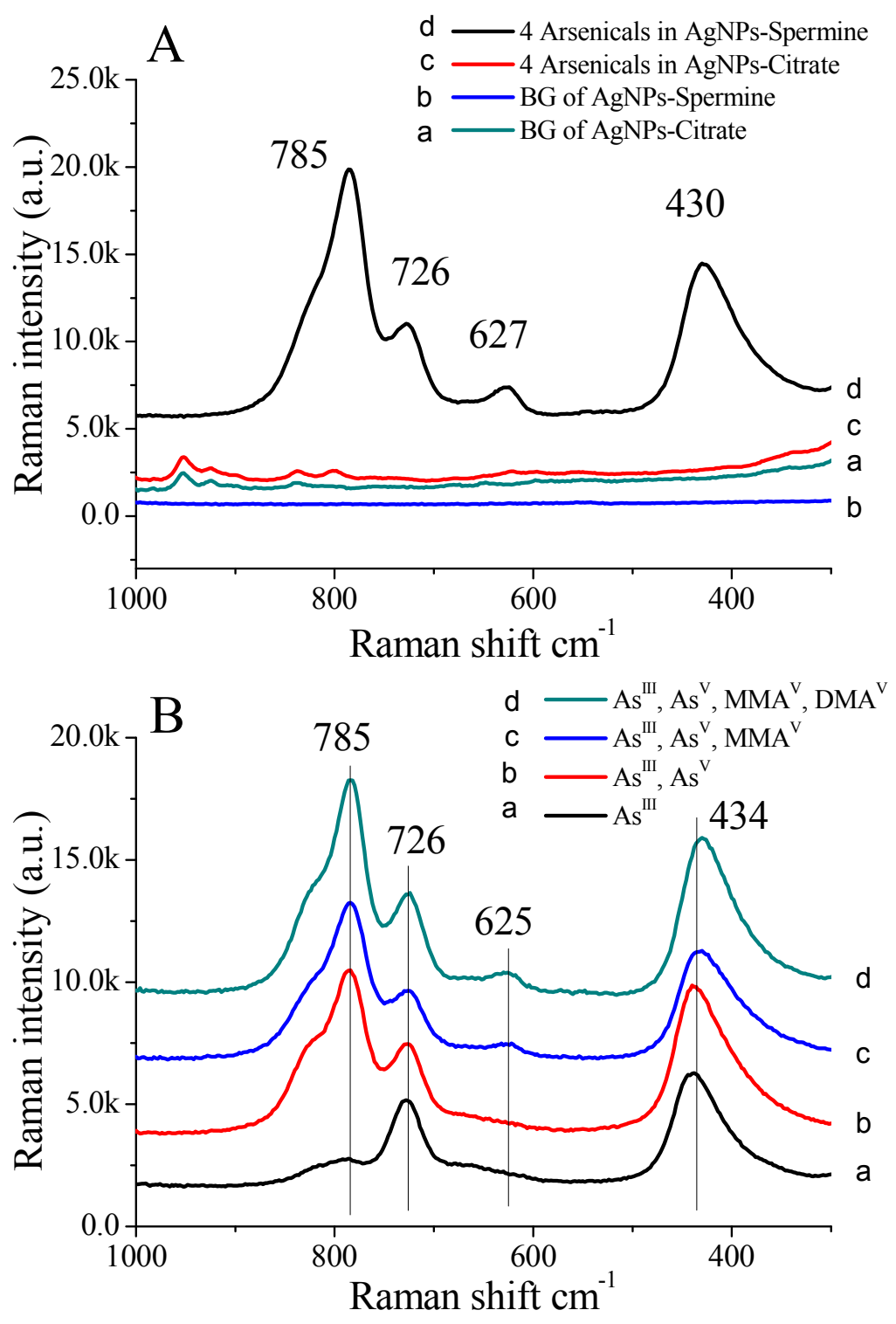

Figure 2.6 SERS spectra of arsenic species in the cell lysate.The SERS signals of arsenic mixture obtained in AgNPs-Citrate (A) and AgNPs-Spermine colloidal suspensions (B). The final concentration of individual arsenic in the AgNPs colloidal suspension was $200 \mathrm{ppb}$. The background signals from AgNPs-Citrate and AgNPsSpermine colloidal suspensions were included for comparison. Figure B illustrates SERS spectra obtained in AgNPs-Spermine suspension with sequential addition of $20 \mu 1$ of 100 ppm individual arsenic species into $180 \mu 1$ of AgNPs-Spermine. 
Substantial differences in SERS measurement of the four arsenicals were observed between AgNPs-Citrate and AgNPs-Spermine suspensions (Fig. 2.6A). The cell lysate at $2 \times 10^{4}$ cells $/ \mathrm{ml}$ in both AgNPs-Citrate and AgNPs-Spermine suspensions did not generate significant background signal in the range of $1000 \sim 300 \mathrm{~cm}^{-1}$, making the SERS measurement of arsenic possible. Intense SERS signals of arsenic were found in AgNPsSpermine in comparison with AgNPs-Citrate. Arsenic fingerprint vibrational bands under the influence of cell lysate were confirmed by the method of sequential addition of individual arsenic standards where charactristic bands associated with each arsenic species appeared with the addition of $\mathrm{As}^{\mathrm{III}}, \mathrm{As}^{\mathrm{V}}$, and $\mathrm{MMA}^{\mathrm{V}} / \mathrm{DMA}^{\mathrm{V}}$ (Fig. 2.6B). The vibrational bands 785,726 , and $627 \mathrm{~cm}^{-1}$ that appeared in the AgNPs-Spermine suspension were the characteristic vibrations of $\mathrm{As}^{\mathrm{V}}, \mathrm{As}^{\mathrm{III}}$, and $\mathrm{MMA}^{\mathrm{V}} / \mathrm{DMA}^{\mathrm{V}}$, respectively. The vibrational band at $430 \mathrm{~cm}^{-1}$ was from both $\mathrm{As}^{\mathrm{III}}$ and $\mathrm{As}^{\mathrm{V}}$, as the sequential addition of $\mathrm{As}^{\mathrm{V}}$ into the solution increased its intensity. Among these vibrational signals, the $\mathrm{As}^{\mathrm{V}}$ signal was the strongest, followed by $\mathrm{As}^{\mathrm{III}}$ and $\mathrm{MMA}^{\mathrm{V}} / \mathrm{DMA}^{\mathrm{V}}$. The relative standard deviations (RSD) for the intensities of these three vibrational band were $11.4,9.8$, and $10.3 \%(\mathrm{n}=3)$, respectively. The RSD values were less than $20 \%$, indicating a relatively reliable SERS measurement [194]. The limit of detections (LOD) calculated based on three times of standard deviation of the blank samples were 1.0, 2.8, and $9.9 \mathrm{ppb}$ for $\mathrm{As}^{\mathrm{V}}$, $\mathrm{As}^{\mathrm{III}}$, and $\mathrm{MMA}^{\mathrm{V}} / \mathrm{DMA}^{\mathrm{V}}$, respectively.

For a better understanding of the SERS response under the influence of cell lysate, $\mathrm{pH}$, size and zeta potential of the AgNPs were monitored under different concentrations of cell lysate (Fig. 2.7). No obvious changes in $\mathrm{pH}$ were observed in the cell lysate at different concentrations in both AgNPs suspensions (data not shown). The size of the 
nanoparticles increased with the amount of cell lysate introduced, especially in the AgNPs-Spermine suspension (Fig. 2.7A), where the size increased from approximately 50 to $90 \mathrm{~nm}$. The zeta potential of the nanoparticles in AgNPs-Spermine maintained a positively charge, while that in AgNPs-Citrate was still negative at different concentrations of cell lysate (Fig. 2.7B). In the AgNPs-Citrate suspension, no significant change in zeta potential was observed with the increase in the concentrations of cell lysate. Considering the fact that under the highest concentration of cell lysate used, the size of AgNPs-Spermine increased sharply, while less of a change appeared for AgNPsCitrate, it was postulated that there were more negatively charged biomolecules than positively charged ones in the cell lysate. The zeta potential of the $2 \times 10^{4}$ cell $/ \mathrm{ml}$ cellular matrix was about $-18 \mathrm{mV}$, indiciating the presence of negatively charged biomolecules in the cell lysate. The size of AgNPs-Spermine increased likely due to the electrostatic attraction in AgNPs-Spermine and as opposed to a repulsive effect in AgNPs-Citrate. This could also explain the changes in zeta potential in the AgNPs suspension, where the zeta potential of AgNPs-Spermine dropped upon introduction of cell lysate due to neutralization of positive charges on the AgNPs surface. The zeta potential remained almost unchanged in the AgNPs-Citrate suspension owing to electrostatic repulsion preventing biomolecules from getting close to the surface of nanoparticles. 

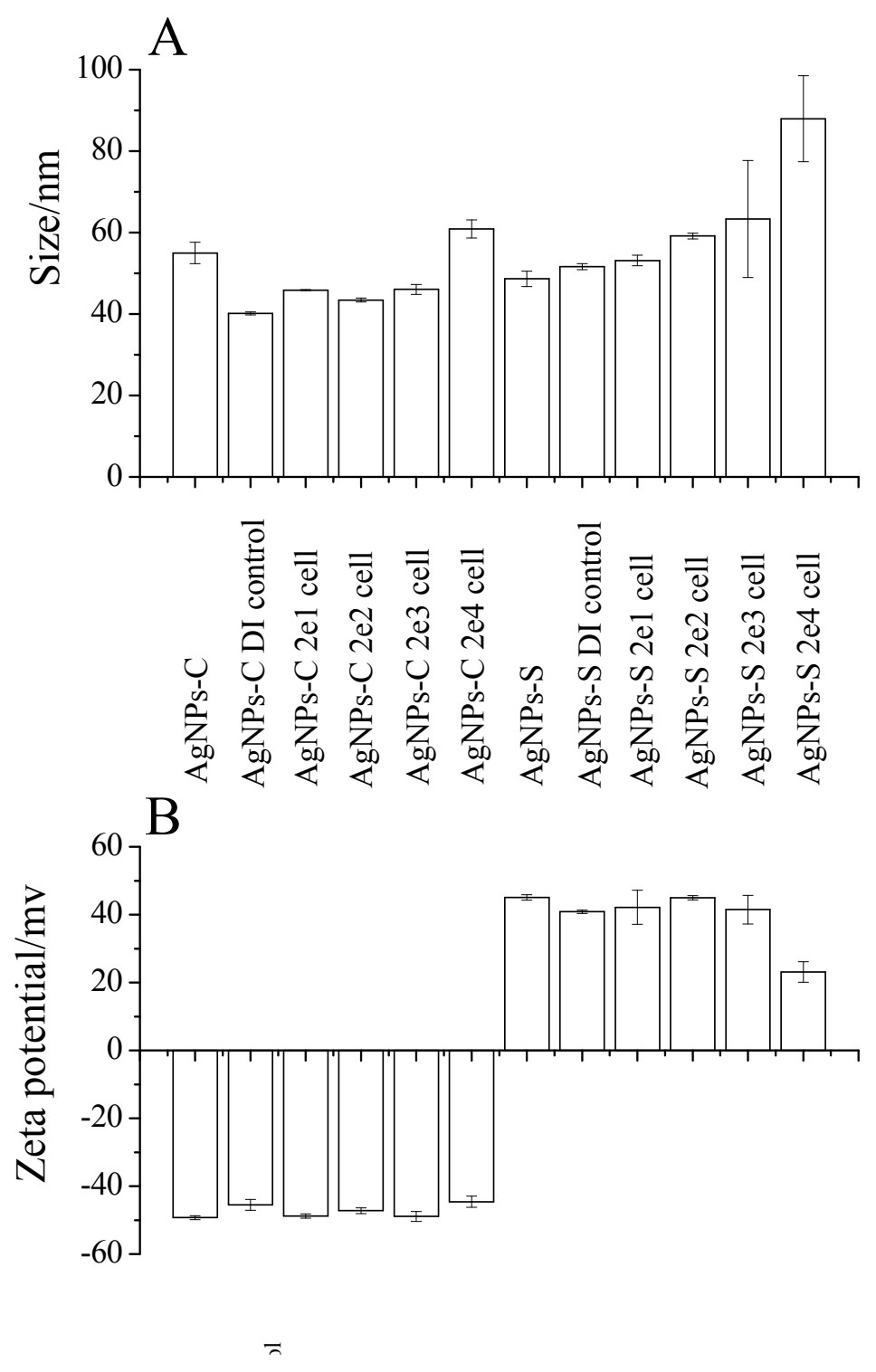

Figure 2.7 Changes in size (A) and zeta potential (B) of AgNPs upon interaction with cell lysate. AgNPs-C and AgNPs-S stand for AgNPs-Citrate and AgNPs-Spermine colloidal suspensions, respectively. For both DI controls, $910 \mu \mathrm{l}$ of AgNPs colloidal suspension was mixed with $90 \mu \mathrm{l}$ of DI water. The AgNPs containing different concentrations of cell lysate were prepared by mixing $910 \mu \mathrm{l}$ of AgNPs colloidal suspension and $90 \mu 1$ of different concentrations of cell lysate. At $P=0.05$ level, changes in size and zeta potential were significant among different cell lysate introduced.

The size and zeta potential of AgNPs-Spermine changed upon exposure to cell lysate, however these changes did not significantly alter the stability of AgNPs in the 
suspension, making the measurement of arsenic using SERS possible. The stability of AgNPs upon interaction with cell lysate was mostly evaluated by the zeta potential, being beyond the range of -30 to $+30 \mathrm{mV}$ under most concentrations of cell lysate introduced. The AgNPs-Spermine positive charge was the key to producing an intense SERS response. In the presence of cell lysate, the AgNPs-Spermine surface could be coated through the formation of a protein corona [144-146, 195], as indicated by the hydrodynamic size of AgNPs-Spermine that increased from 50 to $90 \mathrm{~nm}$. Arsenicals were able to get close to the positively charged surface of AgNPs, despite the formation of a protein corona on the AgNps surface. $\mathrm{As}^{\mathrm{V}}$ was the only $100 \%$ negatively charged among all arsenic species studied using AgNPs-Spermine in the presence of cell lysate (Table S1), it therefore yielded the strongest SERS signal. Another possibility was the aggregation of AgNPs-Spermine upon introduction of the cellular matrix, as the size of AgNPs-Spermine in the presence of $2 \times 10^{4}$ cell $/ \mathrm{ml}$ cellular matrix increased. The aggregation could induce the generation of SERS hot spots, hence increasing the SERS signals. The aggregation could be attributed to surface charge of AgNPs-Spermine being neutralized during the process as evidenced by the dramatically drop in the zeta potential.

\subsection{Conclusions}

Arsenic speciation in a cellular matrix by SERS was studied by employing AgNPs colloidal suspensions. Nanoparticle surface was manipulated by citrate and spermine to form negatively or positively charged AgNPs. Arsenicals showed substantially different SERS responses in the two colloidal suspensions, mainly due to the distinct difference in the interaction between arsenicals and the nanoparticles. This study revealed that saltinduced aggregation is not required for SERS measurement of arsenic in an AgNPs- 
Spermine suspension. However, AgNPs-Citrate required aggregation in order to generate SERS signals. Arsenic speciation in cell lysate could be successfully carried out in an AgNPs-Spermine suspension, while AgNPs-Citrate could not yield significant SERS signals under the experimental conditions used. Both AgNPs colloidal suspensions maintained their original surface charges in the presence of cell lysate. However, a significant drawback of this method was that it was unable to distinguish $\mathrm{MMA}^{\mathrm{V}}$ from $\mathrm{DMA}^{\mathrm{V}}$. This study proved that AgNPs-Spermine colloidal suspension could be a promising SERS substrate for studying arsenic metabolism in a biological matrix, reducing the bias caused by traditional techniques that involve sample extraction and pretreatment. 


\section{Chapter 3}

Employing Coffee Ring Effect on Silver Nanofilm for Arsenic Speciation Study 


\subsection{Abstract}

In this study, an analytical method using a new coffee ring phenomenon was developed and used for arsenic speciation. The coffee ring effect was created via reducing the surface tension of the sessile droplet and enhancing the capillary action generated by closed packing nanoparticles at the three-phase (air, solid and liquid) contact line. For easy discussion, it was named halo coffee ring phenomenon since two concentric circles of coffee ring stain were formed on the surface. The outwards flow caused by the capillary action provides an alternative driving force for separation and the separation mostly depends on the interaction between analyte and the nanofilm surface. Arsenic speciation based on this halo coffee ring phenomenon was demonstrated on a silver nanofilm, which was formed by coating with silver nanoparticles on the glass substrate. Four arsenic species commonly occurred in biological and environmental samples including arsenite, arsenate, monomethylarsonic acid and dimethylarsinic acid, were selected for arsenic speciation analysis using this halo coffee ring effect coupled with surface enhanced Raman Scattering (SERS) technique. Typically, $2 \mu \mathrm{L}$ of arsenic solution was dropped onto the silver nanofilm surface and SERS signals were obtained from the center to edge after complete evaporation. Surfactants were employed to reduce the surface tension and different buffer solutions were used to achieve separation. The separation of arsenic species from the center to edge of halo coffee ring was promising, although peak overlapping for some arsenicals were observed. The method using this new halo coffee ring effect has a great potential to become an alternative and unique technique for separation of arsenic in biological and environmental samples. 


\section{Key words: Coffee ring effect • Halo coffee ring • Arsenic speciation • SERS •}

\section{Silver nanofilm}

\subsection{Introduction}

The coffee ring phenomenon is the ring-shaped coffee stain left on the solid surface when one coffee drop is dried on a solid surface [150]. Deegan and colleagues unveiled the mystery of coffee ring phenomenon and pointed out that when the contact line was pinned everything in the solution will move to the edge with the process of evaporation [150]. With the evaporation of liquid over the liquid drop surface, liquid from the center region would move towards the edge to replenish to lose of liquid since the evaporation rate is higher at the edge than the center. The movement of liquid from the center to the edge is named capillary flow and Deegan pointed out that this capillary flow is not stoppable since surface-tension gradients, solute diffusion, electrostatic and gravity effects - are negligible in ring formation [150]. Currently, a majority of studies involving the coffee ring effect in analytical chemical applications take advantage of the procedure for signal enhancement. For example a drop coating deposition method was employed to concentrate analytes on the coffee ring region and improve detection sensitivity via Raman spectroscopy $[151,152]$. Furthermore, the coffee ring effect can be effectively coupled with surface-enhanced Raman spectroscopy (SERS), only requiring introduction of metallic nanostructure, nanoparticles [131, 153-155] or nanofilms [156]. Because the capillary flow will push everything in the liquid drop towards the edge of the droplet, the accumulated nanoparticles will create hotspots and facilitate SERS signal enhancement $[131,154,157]$. Meanwhile, there are many other techniques developed to enhance SERS signals, e.g., the coffee ring effect and improve the uniformity of 
deposition after the liquid is dried on the surface, including introduction of ellipsoids particles [158] or introduction of hydrosoluble polymer additives into the solution [159].

Although Deegan's paper demonstrated the mechanism of the coffee ring phenomenon, there was a critical matter missing in the paper. The paper did not explain how a liquid drop was pinned on the surface and only stated that it was a geometrical constraint [150]. Later on Ball noted that Thomas Young's famous condition for surface wetting was the key for the coffee ring mechanism [196]. Thomas Young's equation defined the force balance when a liquid droplet forms a hemisphere shape on a solid surface, as the following:

$$
\gamma \mathrm{SV}=\gamma \mathrm{sl}+\gamma \cos \theta_{\mathrm{eq}}
$$

Where $\gamma \mathrm{sv}, \gamma \mathrm{sl}$ and $\gamma$ stand for the solid/air, solid/liquid and liquid/air interfacial tensions and $\theta_{\mathrm{eq}}$ is the contact angle at equilibrium. Typically, when $\gamma \mathrm{sv}<\gamma \mathrm{s} l+\gamma \cos \theta_{\mathrm{eq}}$, the liquid drop will form a spherical cap on the solid surface and when $\gamma \mathrm{sv}>\gamma \mathrm{sl}+\gamma \cos$ $\theta_{\mathrm{eq}}$, the surface will be complete wetted and the liquid will form a thin film on the surface known as a Van der Waals pancake [197]. In Deegan's experiments, the coffee ring phenomenon was observed by placing drops of deionized water (DI) water containing polystyrene microspheres on a surface. These microspheres head towards the contact line during the evaporation [150]. Due to the strong surface tension of water and no surfactant existing in the solution, the liquid drop was held on the surface as a hemisphere cap (pinned on the surface.) This kind of liquid drop on a surface is termed a sessile droplet, which is characterized with a defined wetted area and a contact line [198].

The dynamics of a sessile drop have been extensively studied in order to understand different solute deposition patterns, particle self-assemble during evaporation, 
inkjet printing applications, internal flow directionality, etc [199-202]. Until now it is still considered as a complicated process, and it is suggested that more universal models should be considered for the better understanding of droplet evaporation [203]. However, due to the simplicity, one of the potential applications of sessile drop is the separation capability, which is intriguing. The coffee ring effect has been employed for the successful separation of different sized particles. Owing to geometrical constraints, particles were arranged according to their size at the coffee ring edge $[160,161]$. However, these particles were extremely close to each other along a narrow strip less than $100 \mu \mathrm{m}$ at the edge of the coffee ring as capillary flow drove all solution toward the edge [160]. This study was exciting because it demonstrated the separation capability of coffee ring effect (albeit for particles only) and the sizes of droplets were very small (ranging 0.5 2.0 $\mu \mathrm{L}$ ). By dropping such a small volume of liquid onto the surface, the simplicity of coffee ring-based separation was demonstrated as the separation was merely driven by the capillary flow under the evaporation of the droplet. Again, the strong capillary flow is capable of driving all solutes in the droplet towards the edge of the droplet, regardless of surface-tension gradients, solute diffusion, electrostatic and gravity effects [150]. However, separation based on size might not be applicable towards small molecules, since there is no significant difference in sizes for small molecules.

Currently, there are no reports of applications of the coffee ring effect for the separation of small molecules in the literature. This is probably because potential separation applications based on the coffee ring effect are extremely challenging for the following reasons: 1) The size of the droplet is very small (usually the volume is less than $10 \mu \mathrm{L}$ ); and 2) The capillary flow is too strong to drive all solutes towards the edge. It 
seems that in order to achieve a useful separation using the coffee ring effect, the following requirements should be fulfilled. The liquid in the droplet should be able to overcome the constraint of the droplet and migrate into the peripheral region, otherwise the solute will accumulate at the edge of the droplet. Furthermore, the interaction of small molecules in the droplet with the surface should be strong enough to retain small molecules during the migration. Considering that previous work has found that solution in the droplet could be induced to migrate into the peripheral region via the reduction of surface tension [197, 204] and that enhanced interaction of solutes with the surface should be achievable by replacing the surface with nanoparticles (which would increase the surface area significantly), we reason that it may be possible to realize the separation of small molecules using the coffee ring effect.

Here, we propose to explore the separation capability of the coffee ring effect on the silver nanofilm (AgNF) which is composed of closely packed silver nanoparticles. Surfactants will be introduced into the sessile droplet in order to reduce the surface tension. The strong capillary action, resulting from the closely packed nanoparticles at the droplet edge, should be able to facilitate the migration of solution in the droplet into the peripheral region. The solution migration provides a method for mobilizing analytes in the sessile droplet. This proposed method will be employed to study arsenic speciation of four common existing arsenicals were chosen in order to demonstrate the separation capability, including arsenite $\left(\mathrm{As}^{\mathrm{III}}\right)$, arsenate $\left(\mathrm{As}^{\mathrm{V}}\right)$, monomethylarsonic acid $\left(\mathrm{MMA}^{\mathrm{V}}\right)$ and dimethylarsinic acid $\left(\mathrm{DMA}^{\mathrm{V}}\right)$. SERS will be employed to detect the arsenicals, since the closely packed silver nanoparitcles are capable of enhancing the signals of arsenicals by surface plasmon resonance. 


\subsection{Material and Methods}

\subsubsection{Materials and Chemicals}

Sodium metaarsenite, $98 \%\left(\mathrm{As}^{\mathrm{III}}\right)$, sodium arsenate dibasic, $99 \%\left(\mathrm{As}^{\mathrm{V}}\right)$, cacodylic acid sodium salt, 98\% $\left(\mathrm{DMA}^{\mathrm{V}}\right)$ were purchased from Sigma-Aldrich (St. Louis, MO). Monosodium acid methane arsonate, 99.5\% $\left(\mathrm{MMA}^{\mathrm{V}}\right)$ was purchased from Chem Service, USA. Silver nitrate (99.99\%) was obtained from STREM chemicals (Newburyport, MA). Sodium citrate dihydrate (Granular certified), $\mathrm{NaOH}, \mathrm{HCl}, \mathrm{K}_{2} \mathrm{HPO}_{4}, \mathrm{KH}_{2} \mathrm{PO}_{4}$, ammonium formate, glacial acid were certified A.C.S grade or higher and purchased from Fisher scientific Inc. The phosphate buffer was prepared by mixing equal volume of $0.2 \mathrm{M}$ of $\mathrm{K}_{2} \mathrm{HPO}_{4}$ and $\mathrm{KH}_{2} \mathrm{PO}_{4}$. Sodium dodecyl sulfate (SDS, 99.0\%) and cetrimonium bromide (CTAB, 99.0\%) were purchased from Sigma-Aldrich (St. Louis, MO). (3Aminopropyl)trimethoxysilane (APTMS), was obtained from Sigma Aldrich (St. Louis, MO). All solutions were prepared in deionized water (DI water) (18.2 M $\Omega$, Barnstead Nanopure Diamond) unless with specific indication. All arsenic stock solutions were prepared in DI water at $1000 \mathrm{ppm}$ (as arsenic). Glass microscope slides, purchased from Fisher Scientific (Pittsburgh, PA), were cut into $1 \times 1 \mathrm{~cm}^{2}$ pieces as glass substrates. Small size weighing boats were purchased from Cole-Parmer instrument (Vernon Hills, IL) and $25 \mathrm{ml}$ clear glass vials with caps were purchased from Fishersci (Hampton, NH).

\subsubsection{Instrumentation}

Malvern Zetasizer Nano-ZS (Westborough, MA) was employed to obtain the size and zeta potential of nanoparticles synthesized in the laboratory. The average diameters of hydrodynamic nanoparticles were obtained from the "Z-average" intensity peak as a function of size. UV-vis absorption spectra of silver nanoparticles were obtained from a 
Cary 300 UV-vis spectrometer. All pH measurements were carried out on a Fisher scientific accumet research $\mathrm{AR} 15 \mathrm{pH} / \mathrm{mV} /{ }^{\circ} \mathrm{C}$ meter.

A Veeco multimode nanoscope III D atomic force microscopy (AFM) was employed to obtained surface morphology of the prepared silver nanofilm (AgNF) and to monitor the changes of surface morphology during the cleaning and salinization procedures.

The Raman spectrometer used was the Perkin-Elmer Raman station (Model 400F), which employed a diode laser operating system at $785 \mathrm{~nm}$ with an average power of 100 $\mathrm{mW}$ at the sample and 100-micron spot size. This Raman station was equipped with one Perkin-Elmer microscopy 300, which had a movable stage and a build-in camera monitoring the sample surface. The laser source was focused on the sample on the stage through fiber optics cable connecting from $400 \mathrm{~F}$ to microscopy 300 . Usually, a $20 \times$ optical lens was employed in order to improve sample focus and Raman signal response. One piece of silicon wafer was employed to calibrate the Raman system at a daily basis, and the Raman signal intensity at $522 \mathrm{~cm}^{-1}$ was monitored for calibration purposes. The following SERS measurement parameters used: laser wavelength, $785 \mathrm{~nm}$; exposure time, 1 second; and 4 spectra were averaged per measurement.

\subsubsection{Experimental Procedures}

\subsubsection{Fabrication and characterization of AgNF}

The fabrication of AgNF is consisted of two major steps, first, synthesis of citratecoated silver nanoparticles colloidal suspension (AgNPs-Citrate) and second, immobilization of AgNPs-Citrate onto glass substrates. Silver nanoparticles (AgNPs) were synthesized by reducing silver nitrate with sodium citrate [181]. Briefly, all 
glassware were emerged in the Aqua Regia solution $(\mathrm{HCl} / \mathrm{HNO} 3=3: 1, \mathrm{v} / \mathrm{v})$ overnight and then rinsed with a large amount of tap water and DI water and finally dried in an oven at $80{ }^{\circ} \mathrm{C}$ before use. Then, a $250 \mathrm{ml}$ round bottom flask with $50 \mathrm{ml}$ of $1 \times 10^{-3} \mathrm{M}$ silver nitrate was heated to boil in an oil bath under vigorous stirring with a condenser equipped to reflux. Sodium citrate solution $(2 \mathrm{ml}, 1 \%(\mathrm{w} / \mathrm{v}))$ was added dropwise to the hot solution and kept boiling for one hour to yield the greenish yellow AgNPs-Citrate colloidal suspension. The following properties of the colloidal suspension were obtained after the solution cooled down, including size, surface charge and $\mathrm{pH}$.

The immobilization of AgNPs-Citrate onto glass substrates began with the silanol formation on glass substrates. All glass substrates (about $1 \times 1 \mathrm{~cm}^{2}$ ) were soaked in Aqua Regia solution overnight, and then sonicated successively in concentrated $\mathrm{NaOH}$ solution (2 M) and $\mathrm{HCl}$ solution (2 M) for 2 hours. These glass substrates were thoroughly rinsed with large amount of tap water and DI water to wash away excess acid or base. Finally these glass substrates were dried in an $80{ }^{\circ} \mathrm{C}$ oven prior to use. Then, the AgNF was fabricated following a two-step procedure [166]. The first step was the silanization reaction on glass substrates surface to attach APTMS molecules catalyzed by a diluted acid [205]. APTMS solutions with different concentrations (0.5, 1.0, 2.5, 5.0 and 10.0\% $(\mathrm{v} / \mathrm{v}))$ in anhydrous ethanol were first prepared. Then five pieces of glass substrates were soaked in a $25 \mathrm{ml}$ glass vial containing $10 \mathrm{ml}$ of APTMS solution and $100 \mu \mathrm{L}$ of $1 \mathrm{M} \mathrm{HCl}$ solution for 4 hours on an orbital shaker $(150 \mathrm{rpm})$. After the silanization reaction, the glass substrates were cleaned with anhydrous ethanol under sonication for four times of 3-minutes period in order to remove loosely attached APTMS molecules on the glass surface. These treated glass substrates were then dried by nitrogen and heated in an oven 
at $80{ }^{\circ} \mathrm{C}$ for 30 minutes before the next step. The second step was to immobilize AgNPsCitrate onto the treated glass substrates. AgNPs-Citrate were immobilized by electrostatic interaction with the glass surface, since the amine groups were facing outwards and positively charged in the solution. The high affinity interaction between silver nanoparticles and amine groups shoud further enhance the attachment [166, 206, 207]. Briefly, five pieces of glass substrates prepared from the first step were placed in a small weighing boat with no overlapping among the slides. Next, $5 \mathrm{ml}$ of AgNPs solution was carefully transferred into a weighing boat and the glass slides were submerged in the solution for different time periods ( 4,8 , and 24 hours) on an orbital shaker (50 rpm). In order to see the importance of the silinization procedure, a control experiment was carried by soaking untreated glass substrates in the colloidal suspension for the same period of time. DI water was introduced to make up the evaporation. The resulting glass substrates were then cleaned with DI water for three times, dried under $\mathrm{N}_{2}$ gas, and stored in freezer prior to use.

A UV-vis spectrometer was employed to study surface plasmon resonance of the AgNF with the glass substrate background signal subtracted. Atomic force microscopy (AFM) was used to obtain the surface topology properties of the AgNF, including surface morphology and the AgNF thickness. Two $\mu \mathrm{L}$ of $100 \mathrm{ppm} \mathrm{As}{ }^{\mathrm{III}}$ was dropped onto the AgNF surface, and SERS signals were immediately collected four times at the center region of the droplet by the Raman spectrometer. For comparison, $\mathrm{As}^{\mathrm{III}}$ solution was dropped onto a bare glass slide and Raman measurement was carried out. Preparation parameters of AgNF were optimized including the concentration of APTMS and the soaking period in AgNPs-Citrate solution on the basis of the resulting SERS signals. 


\subsubsection{Arsenicals SERS fingerprint signals on the AgNF}

Stock solutions of arsenic compounds (1000 ppm) were diluted to $100 \mathrm{ppm}$ with DI water or different buffers, including $0.1 \mathrm{M}$ acetic acid $(\mathrm{pH}=2.9), 0.1 \mathrm{M}$ phosphate buffer (potassium salt, $\mathrm{pH}=7.5$ ), and $0.1 \mathrm{M}$ ammonium formate buffer $(\mathrm{pH}=6.9)$ as substock solutions. Typically, $2 \mu \mathrm{L}$ of arsenic solution from sub-stocks were dropped onto the AgNF, and SERS signals were collected randomly at the center region of the sessile droplet. After about 35 minutes of evaporation, the droplet was complete dry and a ring shaped stain was formed on the AgNF. The SERS signals were collected at the edge region of the ring stain.

\subsubsection{Coffee ring formation on the AgNF}

As ${ }^{\text {III }}$ was chosen as the arsenic species to explore the coffee ring effect on the AgNF for arsenic determination by using SERS. Two approaches were used to study this phenomenon, including signals changing with time lapse and signals changing with the distance from the center to edge of coffee ring. To study the change of arsenic SERS signal with time lapse, $2 \mu \mathrm{L}$ of $100 \mathrm{ppm} \mathrm{As}^{\mathrm{III}}$ in DI water was dropped onto the AgNF and SERS signals were collected every 2 minutes under ambient conditions. Meanwhile, the signals changing with distance was carried out by collecting signals from the center to edge with $100 \mu \mathrm{m}$ increment 5, 10, and 15 minutes after the sessile droplet dropped onto the AgNF. The SERS signal response of $\mathrm{As}^{\mathrm{III}}$ on the dry film was poor even at the edge of the coffee ring, thus $\mathrm{MMA}^{\mathrm{V}}$ was employed to study the coffee ring effect from the center to the edge. A complementary experiment was carried out by dropping $2 \mu \mathrm{L}$ of $100 \mathrm{ppm}_{\mathrm{MMA}}^{\mathrm{V}}$ in $0.1 \mathrm{M}$ ammonium formate buffer on the AgNF, and SERS 
measurements were carried out along the radius from the center to the edge of the ring stain with $100 \mu \mathrm{m}$ increments after the complete evaporation.

\subsubsection{Arsenicals speciation on the AgNF}

Mixed arsenic standard solutions containing $\mathrm{As}^{\mathrm{III}}, \mathrm{As}^{\mathrm{V}}, \mathrm{MMA}^{\mathrm{V}}$ and $\mathrm{DMA}^{\mathrm{V}}$ of 100 ppm for each species, were prepared in different buffer solutions, including $0.1 \mathrm{M}$ acetic acid $(\mathrm{pH}=2.9), 0.1 \mathrm{M}$ phosphate buffer $(\mathrm{pH}=7.5)$ and $0.1 \mathrm{M}$ ammonium formate $(\mathrm{pH}=6.9)$, with or without $0.05 \%$ of surfactants, including SDS or CTAB. One drop $(2 \mu \mathrm{L})$ of the solution was dropped onto the AgNF surface and the sessile droplet was allowed to dry on a flat bench under ambient conditions. Different stain patterns were found depending on whether surfactants were applied. The typical coffee ring stain, only having one ring shaped stain, was found for the arsenic solution without surfactants, while the two concentric-ring shaped stain pattern was formed after adding surfactants. Inspired by the previous work of Brochard-Wyart et al. [197, 204], this phenomenon of depositing two concentric-ring shaped stains is named halo coffee ring effect. It is necessary to point out that the three-phase contact line did not move during the evaporation of sessile droplet containing surfactants, only liquid in the sessile droplet permeating into the peripheral region of the sessile droplet. There was a clear boundary between these two concentric rings, named the inner ring (IR) and the outer ring (OR). Typically, SERS measurements on all coffee ring stains were carried out from the center all the way to the OR edge along the radius of the ring and the sampling spots were $100 \mu \mathrm{m}$ increments. For the two concentric-ring SERS measurements around the boundary region, sampling spots at 10 $\mu \mathrm{m}$ inside and outside the boundary were taken as well. 


\subsection{Results and Discussions}

\subsubsection{Optimization of parameters for fabrication and characterization of AgNF}

Monolayer fabrication is a well stuided technique [166, 208, 209] to coat with

functional end groups, such as $-\mathrm{NH}_{2}$ and $-\mathrm{SH}$, on a glass surface. The resulting functionalized glass surface provides a strong binding affinity to gold/silver nanoparticles by electrostatic interaction or metal sulfur bond [210] and thus, uniform nanoparticle film could be prepared. In this study, the glass substrate was modified with APTMS for attaching AgNPs-Citrate onto the surface to prepare AgNF. The $\mathrm{pH}$ of the freshly prepared AgNPs-Citrate colloidal solution was 9.0, and the surface of AgNPs-Citrate was negatively charged according to the zeta potential measurement. Because the $-\mathrm{NH}_{2}$ group of APTMS has a very high $\mathrm{pKa}$ value $(\mathrm{pKa}>10)[211]$, the lone pair electrons on the nitrogen atom should be able to capture a proton in the $\mathrm{pH} 9.0$ solution to form a positively charged $-\mathrm{NH}_{3}{ }^{+}$group [206]. The positively charged surface would facilitate the adsorption of negatively charged AgNPs, and the strong binding between APTMS and AgNPs [206, 207] will further enhance the immobilization of AgNPs-Citrate onto the glass surface.

To optimize the immobilization time of AgNPs-Citrate, glass substrates treated with $2.5 \%$ of APTMS were soaked in the AgNPs-Citrate solution for different period of time (Fig. 3.1A). 

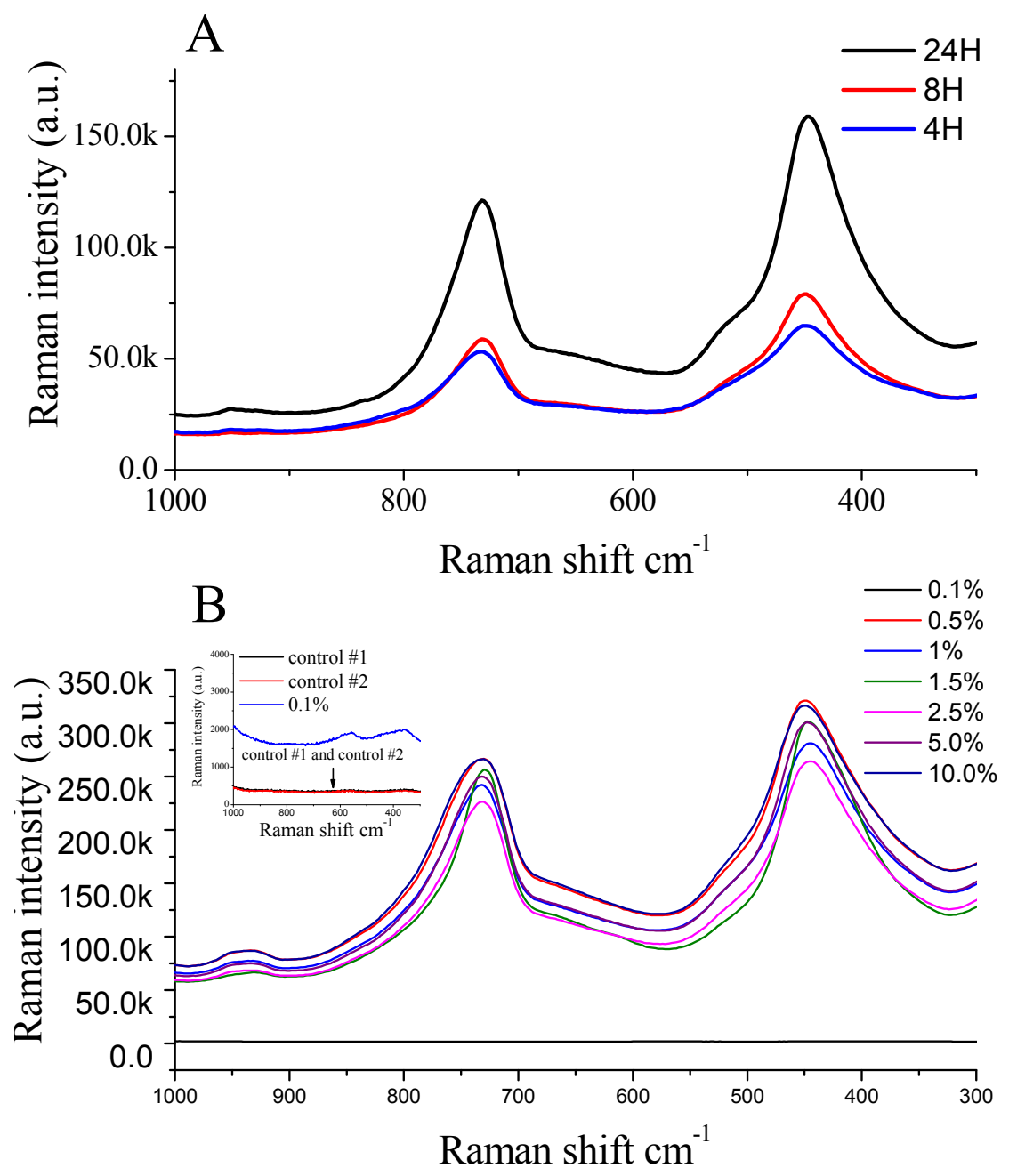

Figure 3.1 Optimization the usage of APTMS. In A, $2 \mu \mathrm{L} 100 \mathrm{ppm}$ As ${ }^{\mathrm{III}}$ SERS signals obtained on AgNF, including two controls (inset), control \#1 was $2 \mu \mathrm{L} \mathrm{As}{ }^{\mathrm{III}}$ on bare glass slides, control \#2 was $2 \mu \mathrm{L} \mathrm{As}{ }^{\mathrm{III}}$ on glass slide without salinization procedure. B, SERS intensity of vibrational band at $732 \mathrm{~cm}^{-1}$, the average intensity was calculated from 4 individual measurements.

It was found that 24 hours of immobilization yields the strongest signal response for $2 \mu \mathrm{L}$ of $100 \mathrm{ppm} \mathrm{As}^{\mathrm{III}}$ in DI water, and less strong signal was obtained after immobilization of 8 hours and the weakest signal was obtained for the reaction of 4 hours. 
The time of immobilization was selected as 8 hours in the further experiments for the sake of saving time. The amounts of APTMS needed were optimized (Fig. 3.1B). No SERS signals were observed from the two control experiments, including the bare glass slide and the glass slide without APTMS treatment and only minimum signals were obtained by using $0.1 \%$ of APTMS. Strong signals were yielded by using 0.5 to $10.0 \%$ of APTMS. These results indicated that modification of the glass surface using APTMS was the key factor for the successful fabrication of the AgNF. In addition, it seems that $0.5 \%$ of APTMS would be sufficient for the treatment of glass substrates. Eventually, $1 \%$ of APTMS was employed for the following experiments.
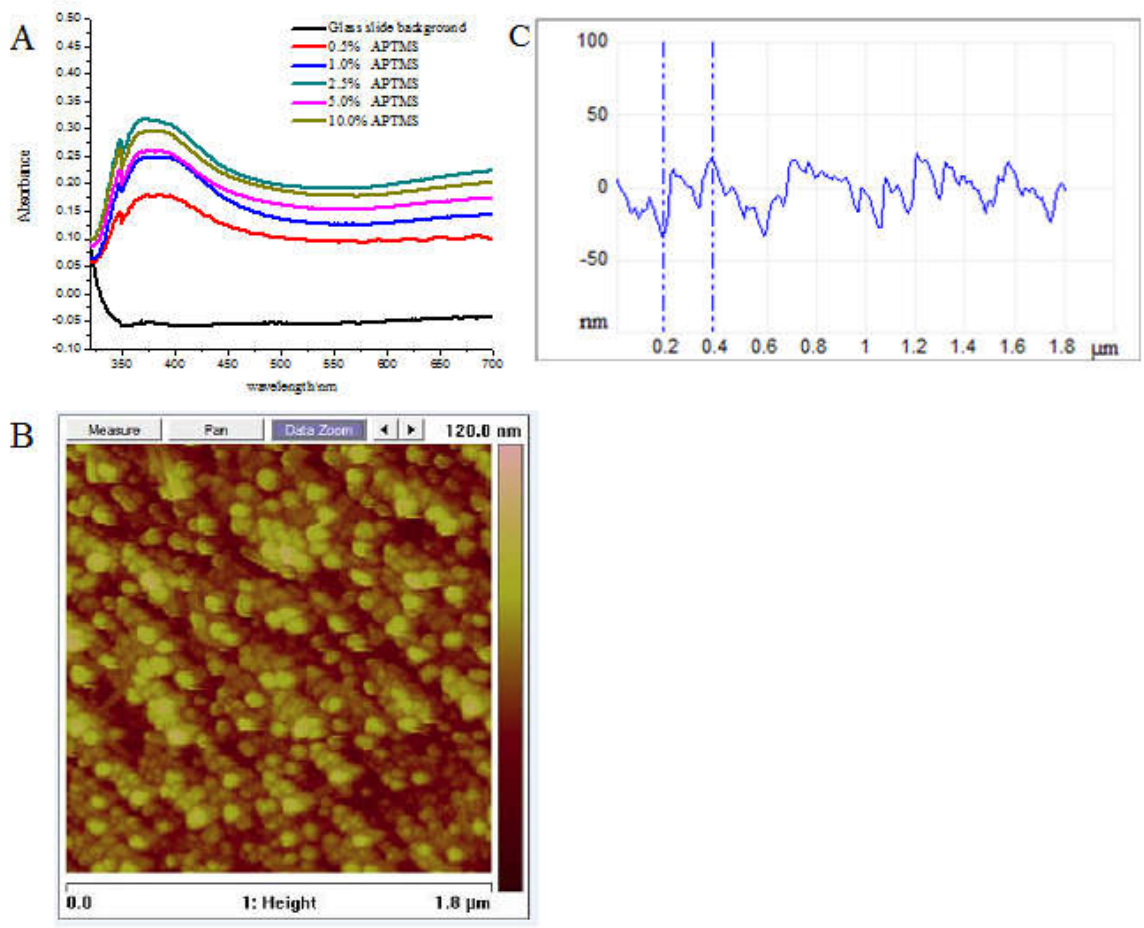

Figure 3.2 Properties of the AgNF. A, UV-vis absorbance of AgNF fabricated using different concentration of APTMS; B, the typical AgNF surface morphology using AFM depth imagine; $\mathrm{C}$, the cross-section profile of the AgNF and the vertical distance was the distance between the valley and the peak as the two dash lines indicated. 
UV-vis spectrum was obtained to compare the surface plasmon resonance of the AgNF fabricated using different amount of APTMS (Fig. 3.2A), and the AgNF surface morphology was studied using AFM (Fig. 3.2B). The intensity of surface plasmon resonance should be in positive correlation with the density of AgNPs immobilized on the AgNF. As shown in Fig. 3.2A, the surface plasmon resonance of AgNF increased with higher concentration of APTMS in general with an absorbance exception of the AgNF fabricated using 2.5\% APTMS, which was the greatest among all AgNF. This observation of decline in AgNF surface plsmon resonance with APTMS more than 2.5\% could be attributed to the limited area of glass substrates. When occupation of alkylsilanes on the glass substrates reached the threshold, excessive APTMS could no longer improve the attachment of AgNPs-Citrate. Once the amount of APTMS was over the threshold, excessive APTMS molecules could react with the amine groups on the surface and thus reduce the binding capacity of AgNPs. As shown in Fig. 3.2B, the AgNF surface was coated thoroughly with AgNPs as bright dots, however, the AgNPs arrangement was not well organized. AFM cross-section of the sample is shown in Fig. 3.2C. Ten random sections were selected in order to calculate the mean vertical distance of the AgNF thickness, which was found to be $48.7 \pm 6.7 \mathrm{~nm}$. The average size of AgNPs was about $50 \mathrm{~nm}$ according to the z-average measurement by Zetasizer suggesting that the AgNF formed a monolayer film.

\subsubsection{SERS response of arsenicals on the AgNF}

The typical fingerprint SERS signals of different arsenicals including $\mathrm{As}^{\mathrm{III}}, \mathrm{As}^{\mathrm{V}}$, $\mathrm{MMA}^{\mathrm{V}}$ and $\mathrm{DMA}^{\mathrm{V}}$ in different solutions are shown in Fig. 3.3, as well as background 
signals of different AgNF surface were studied in the fingerprint signals range (1000 300 $\left.\mathrm{cm}^{-1}\right)$ in Fig. 3.4.
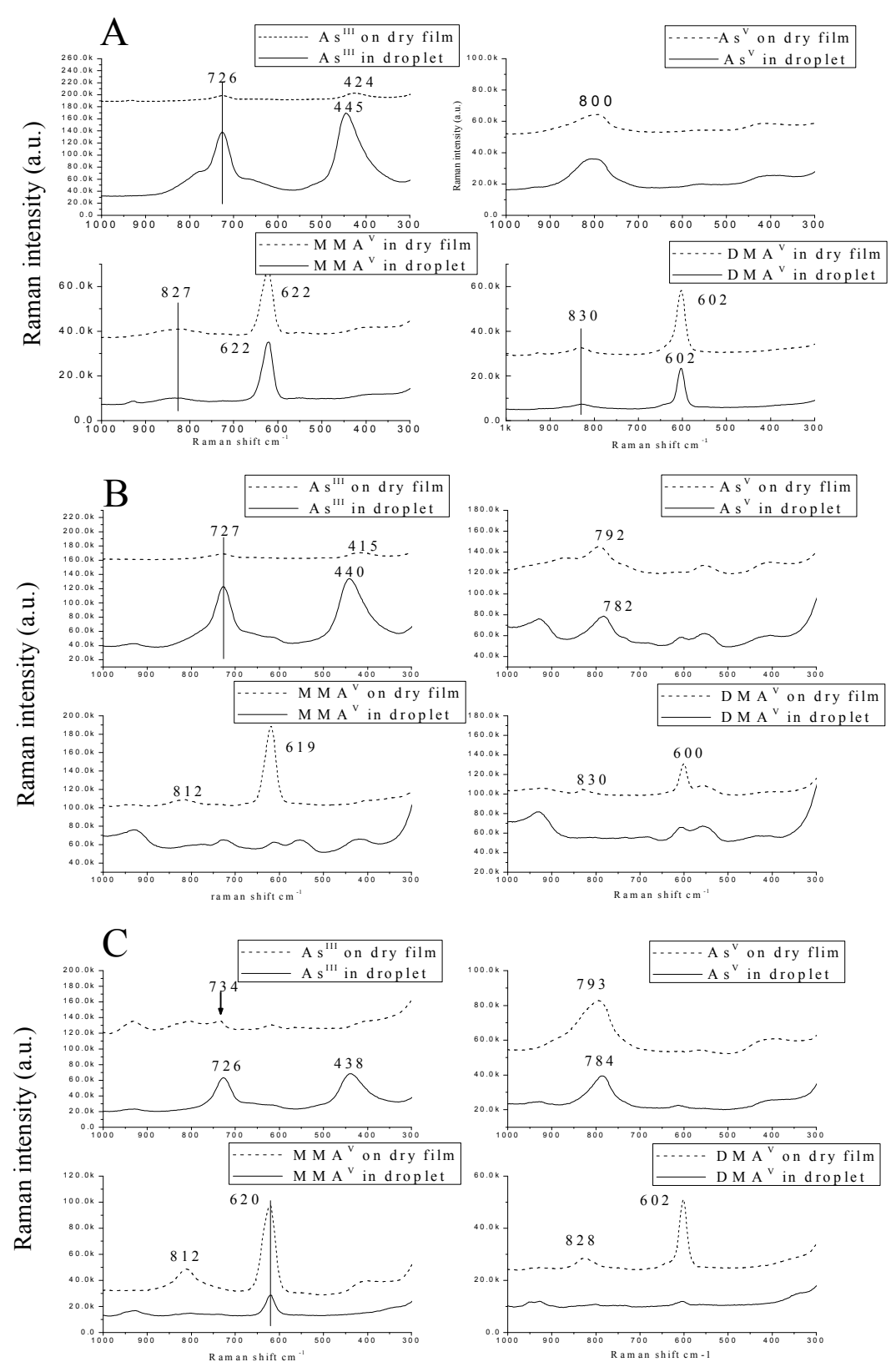

Figure 3.3 Individual arsenic SERS signals on the AgNF as droplet (as black curves) and at the edge of dry film (as red curves). All signals were obtained from $2 \mu \mathrm{L}$ of $100 \mathrm{ppm}$ arsenic solutions in different buffers, $\mathrm{A}-0.1 \mathrm{M}$ acetic acid $(\mathrm{pH}=2.9), \mathrm{B}-0.1 \mathrm{M}$ phosphate buffer ( $\mathrm{pH}=7.5), \mathrm{C}-0.1 \mathrm{M}$ ammonium formate buffer $(\mathrm{pH}=6.9)$. 

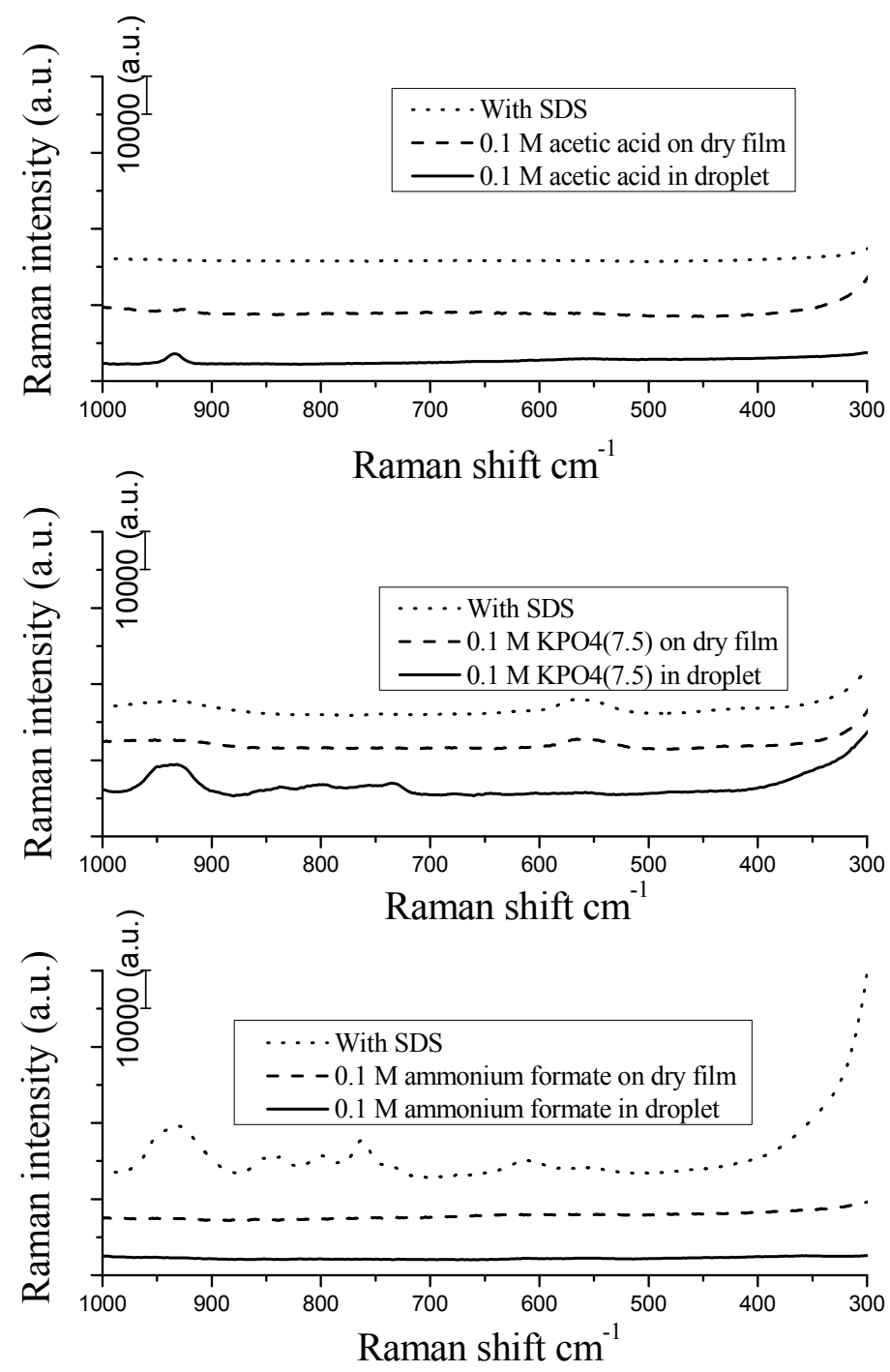

Figure 3.4 Typical SERS background signals from different buffer system with or without SDS including. $\mathrm{A}-0.1 \mathrm{M}$ acetic acid; $\mathrm{B}-0.1 \mathrm{M}$ phosphate buffer (potassium salt, $\mathrm{pH}=7.5) ; \mathrm{C}-0.1 \mathrm{M}$ ammonium formate.

Signals were collected at the center of droplet once $2 \mu \mathrm{L}$ of $100 \mathrm{ppm}$ arsenic solution was placed on the AgNF. Because of the coffee ring effect, signals were collected close to the edge region of coffee ring stain after complete evaporation of the sessile droplets. There was no significant interference in the Raman shift range 1000 300 
$\mathrm{cm}^{-1}$ from these three buffers employed here (Fig. 3.4). In 0.1 M acetic acid (Fig. 3.3A), a characteristic vibrational band at $726 \mathrm{~cm}^{-1}$ for $\mathrm{As}^{\mathrm{III}}$ was observed, which was resulted from As-O stretch mode [184]. The band at $445 \mathrm{~cm}^{-1}$ was not reported in literature, and no attempt was made to identify and assign this band. In addition, it was observed that the band at $445 \mathrm{~cm}^{-1}$ was always present along with the band at $726 \mathrm{~cm}^{-1}$ for $\mathrm{As}^{\mathrm{III}}$ and usually with stronger intensity. After the sessile droplet dried completely, signal intensities of both bands reduced significantly but were still distinguishable with the unassigned vibrational band shifting to a low vibrational number at $424 \mathrm{~cm}^{-1}$. When $\mathrm{As}{ }^{\mathrm{III}}$ was present in the sessile droplet, $\mathrm{As}^{\mathrm{III}}$ could get really close to the AgNF surface due to diffusion and thus the signal intensity was very strong. Although the evaporation of solution should be able to bring $\mathrm{As}^{\mathrm{III}}$ molecules significantly close to the AgNF, the SERS signal dropped dramatically unexpectedly. The dramatic changes in SERS signals between in the droplet or on the dry film could be due to the fact that $\mathrm{As}^{\mathrm{III}}$ has a pKa value of 9.2 and it is always protonated in the acetic acid buffer. The fully protonated $\mathrm{As}{ }^{\mathrm{III}}$ can prevent the complexation of $\mathrm{As}^{\mathrm{III}}$ with the AgNF surface. For $\mathrm{As}^{\mathrm{V}}$, it had a fingerprint signal at 800 $\mathrm{cm}^{-1}$ in the sessile droplet or on the dry film and this could be assigned to the As-O symmetric vibration [186]. The superposition stretch of $v 2$ and $v 5$ of the arsenate molecule was not distinguishable in this buffer solution, which usually appears around $410 \mathrm{~cm}^{-1}[132]$. Due to the similarity in structure for the two organoarsneicals, $\mathrm{MMA}^{\mathrm{V}}$ and DMA ${ }^{\mathrm{V}}$, being different by one methyl group, their SERS signal profiles were highly resemble. MMA ${ }^{\mathrm{V}}$ showed one sharp vibrational band at $622 \mathrm{~cm}^{-1}$ due to the As-C stretch (Fig. 3.3C) and one board vibrational band at $820 \mathrm{~cm}^{-1}$, which should be assigned to the vibrational of As-O [188]. For $\mathrm{DMA}^{\mathrm{V}}$, the symmetric stretching of As-C was at 602 
$\mathrm{cm}^{-1}$ since $\mathrm{DMA}^{\mathrm{V}}$ has two As-C bonds, while the asymmetric As-O stretching was found at $830 \mathrm{~cm}^{-1}[135]$. Being the only trivalent arsenic compound among these four arsenicals, $\mathrm{As}{ }^{\mathrm{III}}$ had a poor signal response on the dry film, while the rest had strong signal responses in both conditions. As mentioned previously, the possible reason could be that $\mathrm{As}^{\mathrm{III}}$ could not form complexation with the AgNF surface while the others could.

Arsenicals SERS signals behaviors were highly similar between phosphate and ammonium formate buffers, since there is minor difference in their $\mathrm{pH}$. In the phosphate buffer (Fig. 3.3B), a slightly different in signal for $\mathrm{As}^{\mathrm{III}}$ was found with very small changing in vibrational frequency and the signal intensity dropped significantly on the dry film in comparison with that in droplet, while the vibrational frequency of $\mathrm{As}^{\mathrm{V}}$ changed much more than $\mathrm{As}^{\mathrm{III}}$, from 800 to $782 \mathrm{~cm}^{-1}$ in the droplet and to $792 \mathrm{~cm}^{-1}$ on the dry film. For the two organoarsenicals, they had poor signal response in the droplet but relatively strong signals on the dry film, being different from their signals in the acetic acid buffer. In the ammonium formate buffer (Fig. 3.3C), these four arsenicals had similar signal response as in the phosphate buffer with minor differences in vibrational frequency with an exception for $\mathrm{MMA}^{\mathrm{V}}$. $\mathrm{MMA}^{\mathrm{V}}$ had very strong vibrational intensity on the dry film, but only distinguishable signals in the droplet. When comparing the SERS signal of these two organoarsenicals in acetic acid with the rest two buffers, it was observed that they all have strong signal responses on the dry film, however, the SERS signal responses in the sessile droplet were completely different. In the acetic acid buffer, they have strong signals in the sessile droplet, which was almost the same as on the dry film. However, the signals were fairly poor or barely distinguishable with the $\mathrm{pH}$ increase 
in other two buffers. The possible reason could be the deprotonation of organoarsenicals and the negatively charged AgNF surface in buffers with high $\mathrm{pH}$.

\subsubsection{Changes in arsenic SERS signals during evaporation of solvent}

The essential of the coffee ring effect is the enrich capability during the evaporation of the sessile droplet. According to Deegan's work in 1997 [150], all solution will be driven to the edge of the sessile droplet as long as the contact line is pinned. Here two approaches were employed to validate this statement for arsenic analysis by SERS. First, the time lapse for signal changes of $\mathrm{As}^{\mathrm{III}}$ was obtained (Fig. 3.5A). SERS signals of $\mathrm{As}^{\mathrm{III}}$ were decreasing with the time at the center of the droplet, indicating that the concentration of $\mathrm{As}^{\mathrm{III}}$ at the center region reduced with time. This observation should be due to the migration of $\mathrm{As}^{\mathrm{III}}$ outwards from the center of the sessile droplet during the evaporation. The second approach was to collect SERS signals along the center to edge of the dry coffee ring. However, $\mathrm{As}^{\mathrm{III}}$ had a poor SERS signal response on the dry film as mentioned in the previous section. Therefore, $A s^{\text {III }}$ SERS measurements were carried out before the sessile droplet dried completely and the SERS signals were collected from the center to the edge of the droplet with $100 \mu \mathrm{m}$ increment in 5,10 and 15 minutes after the $\mathrm{As}^{\mathrm{III}}$ solution being dropped onto the AgNF surface. Similar results were obtained with 5, 10 and 15 minutes of drying. 

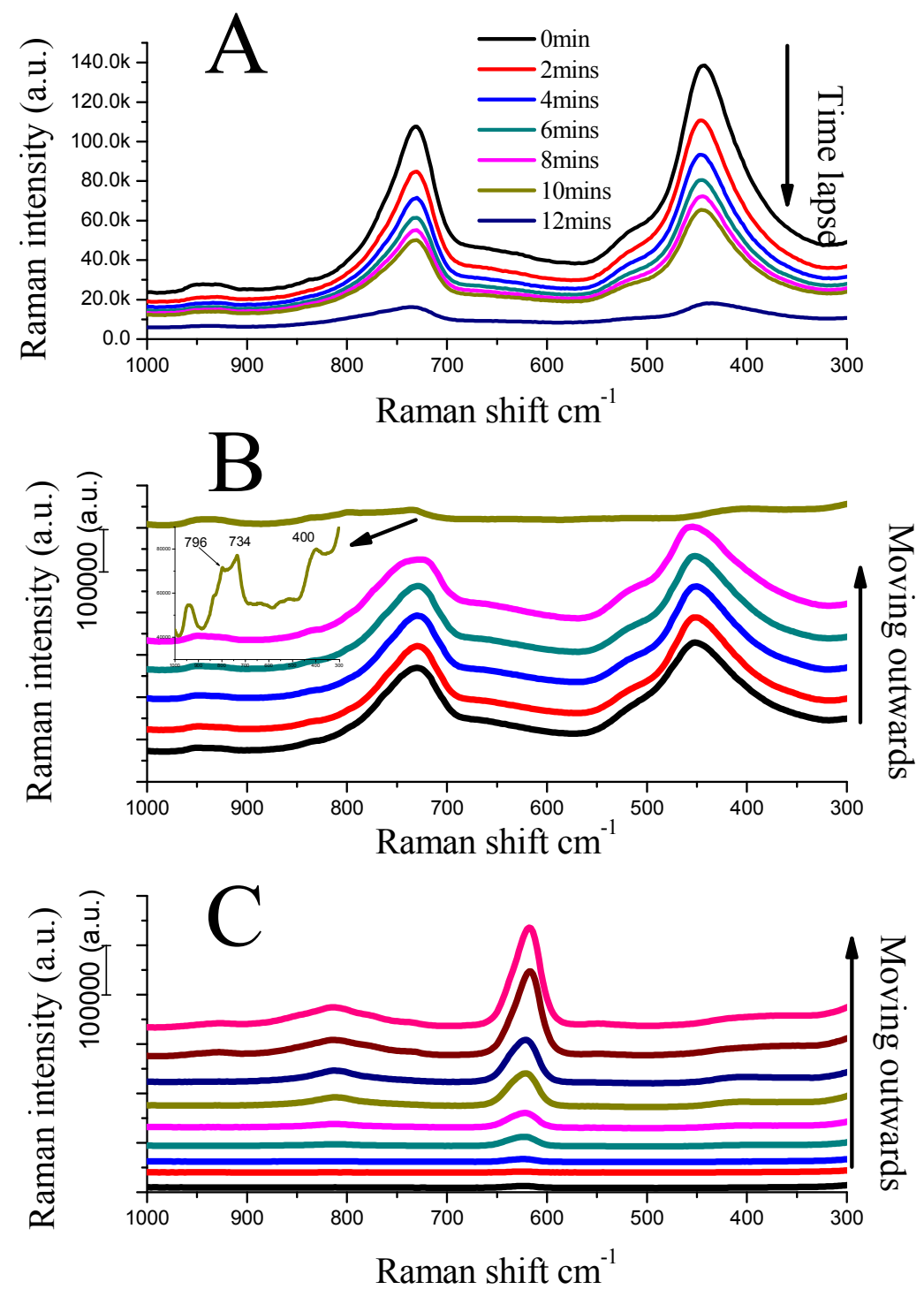

Figure 3.5 The typical coffee ring signal profile of aresnicals on the AgNF. A-Time lapse SERS signals of $2 \mu \mathrm{L} 100 \mathrm{ppm} \mathrm{As}{ }^{\mathrm{III}}$ (prepared in DI water) droplet on AgNF; $\mathrm{B}-\mathrm{As}{ }^{\mathrm{III}}$ SERS signal from the center to edge after $2 \mu \mathrm{L} 100 \mathrm{ppm}$ As ${ }^{\mathrm{III}}$ was dropped onto the AgNF for 5 minutes. Note, it took around 35 minutes for a $2 \mu \mathrm{L}$ droplet to evaporate totally on the AgNF.

The results of 5-minute of drying are shown in Fig 4.5B as an example. It was found that there was no significant difference in signal intensity along the radius of the 
droplet, except a dramatic change in the signal profile at the edge where $\mathrm{As}^{\mathrm{V}}$ fingerprint signals were observed. Also, we noticed that the droplet was dried faster during the SERS measurement than that in the air. Therefore, heat generated from laser irradiation could cause the oxidation of $\mathrm{As}^{\mathrm{III}}$ to $\mathrm{As}^{\mathrm{V}}$. Although the signals maintained almost the same along the radius of the sessile droplet, the concentration of $\mathrm{As}^{\mathrm{III}}$ should be higher near the edge than that of in the center. The reason for the lacking in the signal increase with distance from the center is unknown. Another experiment using $\mathrm{MMA}^{\mathrm{V}}$ in ammonium formate buffer was carried out to observe the migration of arsenic during the evaporation (Fig. 3.5C). It was found that the SERS signal intensity was increased with the sampling spot moving outwards and the outmost sampling spot has the strongest signal intensity. It was clear that the coffee ring effect drives all solute towards the edge of the sessile droplet during the evaporation.

\subsubsection{Arsenic speciation on the AgNF employing the coffee ring effects}

\subsubsection{Arsenic speciation on the AgNF without using surfactants}

It was hypothesized that arsenic speciation could be carried out using the outwards capillary flow as a mobile phase and the AgNF area covered by the sessile droplet covering as the stationary phase. Nano-size particles were coated and packed closely on the surface of glass substrate and this property of the AgNF provided the adsorption/desorption interactions with the arsenicals. During the migration of the arsenicals in the droplet toward the edge driven by the capillary flow, different arsenic species might be separated because of different affinities to the AgNF surface. By monitoring the SERS signals from the center to the edge, the separation of arsenic species could be detected, as the fingerprint SERS signals representative of different arsenicals 
should appear in the different regions on the AgNF surface. In order to examine the possibility of using this coffee-ring effect for arsenic speciation, arsenic species were prepared in different buffers, including $0.1 \mathrm{M}$ acetic acid $(\mathrm{pH}=2.9), 0.1 \mathrm{M}$ phosphate buffer $(\mathrm{pH}=7.5), 0.1 \mathrm{M}$ ammonium formate buffer $(\mathrm{pH}=6.9)$, which have shown to have insignficant background signals in the 1000 300 $\mathrm{cm}^{-1}$ range. SERS signals were obtained along the radius from the center to the edge of coffee ring after the sessile droplet was dry, and the results are shown in Fig. 3.6.
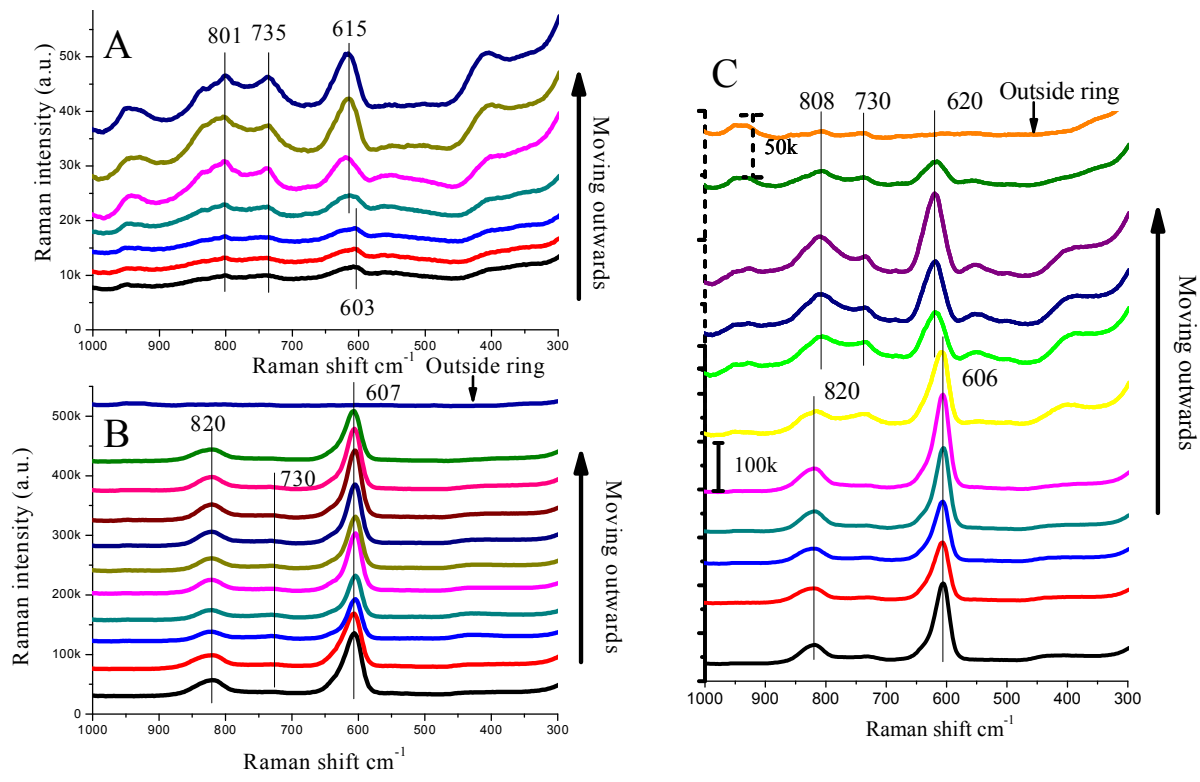

Figure 3.6 Arsenic speciation based on regular coffee ring phenomenon in different buffer solutions.

A $-0.1 \mathrm{M}$ acetic acid $(\mathrm{pH}=2.9), \mathrm{B}-0.1 \mathrm{M}$ phosphate buffer $(\mathrm{pH}=7.5), \mathrm{C}-0.1 \mathrm{M}$ ammonium formate buffer $(\mathrm{pH}=6.9)$. Note in $\mathrm{C}$, the scale bar of upper section (starts with the yellow curve) is different from lower section.

There were significant differences in arsenic fingerprint signal profiles by using different buffers. For the acetic acid buffer (Fig. 3.6A), the center region had low intensity of fingerprint SERS signals of 603,735 and $801 \mathrm{~cm}^{-1}$, standing for the As-C 
stretching from $\mathrm{DMA}^{\mathrm{V}}$, As- $\mathrm{O}$ stretching from $\mathrm{As}^{\mathrm{III}}$ and $\mathrm{As}-\mathrm{O}$ symmetric stretching from $\mathrm{As}^{\mathrm{V}}$, respectively. On the contrary, the SERS signals intensity increased dramatically toward the edge, with the strongest signals found at the outmost edge. At the edge region, the As-C vibrational frequency shifted to $615 \mathrm{~cm}^{-1}$ which was originated from $603 \mathrm{~cm}^{-1}$ at the center. This vibrational band of $615 \mathrm{~cm}^{-1}$ might be due to the overlap of the two organoarsenicals $\left(\mathrm{MMA}^{\mathrm{V}}\right.$ and $\mathrm{DMA}^{\mathrm{V}}$ ), because this vibrational frequency was between the As-C vibrational frequencies of $\mathrm{MMA}^{\mathrm{V}}$ and $\mathrm{DMA}^{\mathrm{V}}$. Despite the discrepancies in the SERS signals between the center and the edge, the separation of arsenic species was not observed in the acetic acid buffer, instead the arsenicals were stacked at the edge region, as multiple arsenicals fingerprint signals (and the strongest signals) were found at the edge.

For the phosphate buffer (Fig. 3.6B), three arsenic fingerprint signals were found, including 607, 730 and $820 \mathrm{~cm}^{-1}$, indicative of As-C vibration, As-O stretching from $\mathrm{As}^{\mathrm{III}}$ and $\mathrm{As}-\mathrm{O}$ stretching from $\mathrm{MMA}^{\mathrm{V}}$, respectively. Compared to the results using the acetic acid buffer, the signal intensity at $820 \mathrm{~cm}^{-1}$ was higher for the organoarsenical using phosphate buffer. No $\mathrm{As}^{\mathrm{V}}$ fingerprint signals were found at any spot on the dry film due to possible overlap with the strong organoarsenic signals. It is worth noting that the signal intensity in phosphate buffer maintained stable along the radius with some minor decreasing at the edge, which was against the coffee ring effect. One of possible reasons could be the binding reactions between AgNPs on the AgNF and arsenicals, which occurred because of the high $\mathrm{pH}$ of phosphate buffer resulting in the deprotonation of arsenicals. At this $\mathrm{pH}$, the binding of arsenicals with AgNPs could be strong enough to retain arsenicals at the center region. 
For the ammonium formate buffer, the SERS signal intensity in the center region was stronger than that in the edge region, as shown by the scale bar changing in Fig. 3.6C. Before the yellow curve, there were two fingerprint signals $\left(820\right.$ and $\left.606 \mathrm{~cm}^{-1}\right)$ found close to the center, indicating that both organoarsenicals were present in this region, since neither of these two signals could be solely from $\mathrm{MMA}^{\mathrm{V}}$ or $\mathrm{DMA}^{\mathrm{V}}$. After moving outwards, three vibrational bands were detected, including 808,730 and $620 \mathrm{~cm}^{-1}$. The peak at $730 \mathrm{~cm}^{-1}$ can be assigned to $A \mathrm{~s}^{\mathrm{III}}$ and the signal at $620 \mathrm{~cm}^{-1}$ should be assigned to As-C in $\mathrm{MMA}^{\mathrm{V}}$. The clear red shift for the As-C vibrational band from 606 in the center to $620 \mathrm{~cm}^{-1}$ at the edge of the coffee ring suggests that separation of the organoarsenicals happened. No $\mathrm{As}^{\mathrm{V}}$ fingerprint signals found in this buffer, but there was a band at $808 \mathrm{~cm}^{-1}$ at the edge region. It was believed that this $808 \mathrm{~cm}^{-1}$ band was not from $\mathrm{As}^{\mathrm{V}}$ or organoarsenicals, instead was the result of vibrational frequency resonance. Probably due to the presence of this $808 \mathrm{~cm}^{-1}$ band in ammonium formate buffer, $\mathrm{As}^{\mathrm{V}}$ was not detected successfully.

Overall, the results using these three buffers suggest that, without further modifications on the experimental conditions, it was extremely difficult to accomplish arsenic speciation by evaporating a $2 \mu \mathrm{L}$ sessile droplet on the AgNF. According to the observations, the capillary flow generated during the evaporation could be strong enough that drove all solutes in the sessile droplet towards the edge of coffee ring and thus all arsenicals were stacked at the edge of coffee ring. Although fingerprint signals of multiple arsenicals were observed at the edge, no separation was achieved under this condition. Strong interactions between arsenicals and the AgNF surface were also observed, resulting in that arsenicals SERS intensities are stronger at the center region 
than the edge region. The ideal arsenic speciation requires the redistribution of arsenic species of interest along the coffee ring radius through modifying experimental conditions, e.g., addition of surfactants (see below).

3.4.4.2 Arsenic speciation on the AgNF using coffee ring effect under the influence of surfactants

In Deegan's paper about coffee ring mechanism, it was pointed out that surfacetension gradients, solute diffusion, electrostatic and gravity effects are negligible in ring formation [150]. This statement indicates that it could not be effective trying to separate arsenic species during the formation of coffee ring without reshaping the ring. Inspired by previous work [197, 204], the coffee ring phenomenon under the influence of surfactants was explored for potential separation of arsenic species. A small amount of surfactant was introduced into the solution in order to reduce the surface tension, and the liquid in the sessile droplet would be migrated into the peripheral area with the reduced surface tension and strong capillary action from the AgNF surface. The strong capillary action was resulted from the closely packed AgNPs on the AgNF surface. When the liquid in the sessile droplet started permeating into the peripheral region outside the sessile droplet, different arsenicals should be able to move with the capillary flow and migrate different distances on the AgNF surface because of the varying strengths in the interactions of different arsenicals with the AgNPs. As the contact line of the droplet did not move during the evaporation of the droplet, two concentric rings stains were left on the AgNF surface, as shown in Fig. 3.7. 


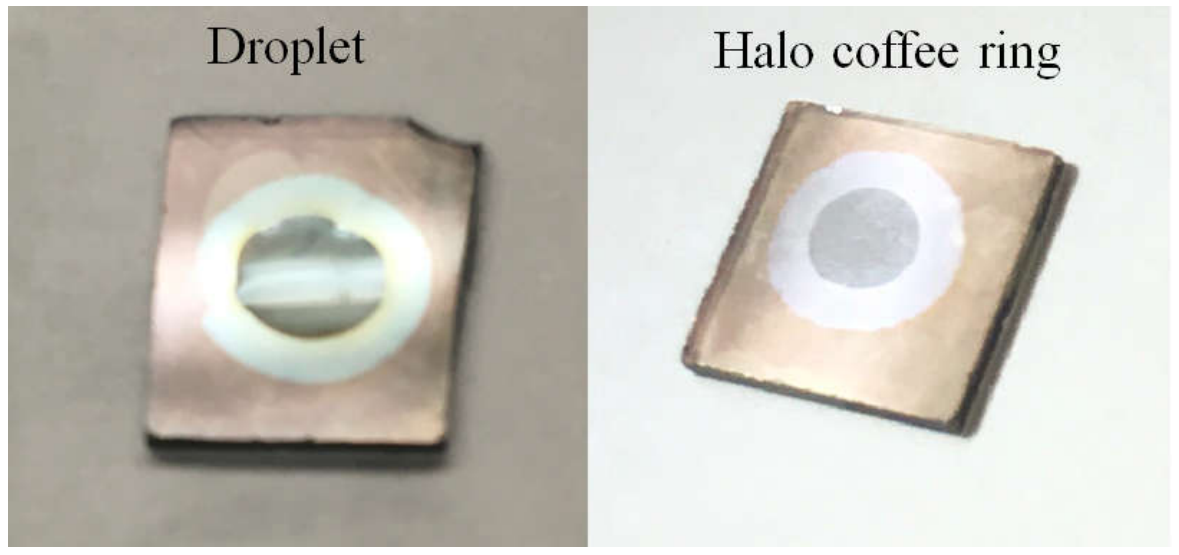

Figure 3.7 Typical halo coffee ring phenomenon as droplet and as dry film using the phosphate buffer system containing $0.05 \%$ SDS.

The selection of buffers would play an important role for the potential arsenic separation using this halo coffee ring effect, since the buffer would determine the $\mathrm{pH}$ of the sessile droplet and at the meantime affect the adsorption of arsenicals on the AgNF during the evaporation. The key to choose an effective buffer and a surfactant for this halo coffee ring effect mainly depends on the background signals and the SERS signals of individual arsenicals in the buffer after the evaporation. Three buffer systems were tested due to their low background interferences with arsenicals, including $0.1 \mathrm{M}$ acetic acid ( $\mathrm{pH}=2.9), 0.1 \mathrm{M}$ phosphate buffer (potassium salt, $\mathrm{pH}=7.5$ ) and $0.1 \mathrm{M}$ ammonium formate $(\mathrm{pH}=6.9)$. Regarding the surfactants, CTAB strongly adsorbed onto the AgNF and caused strong background signal, and therefore CTAB was not suitable for the generation of halo coffee ring (see details in Fig. 3.8). 


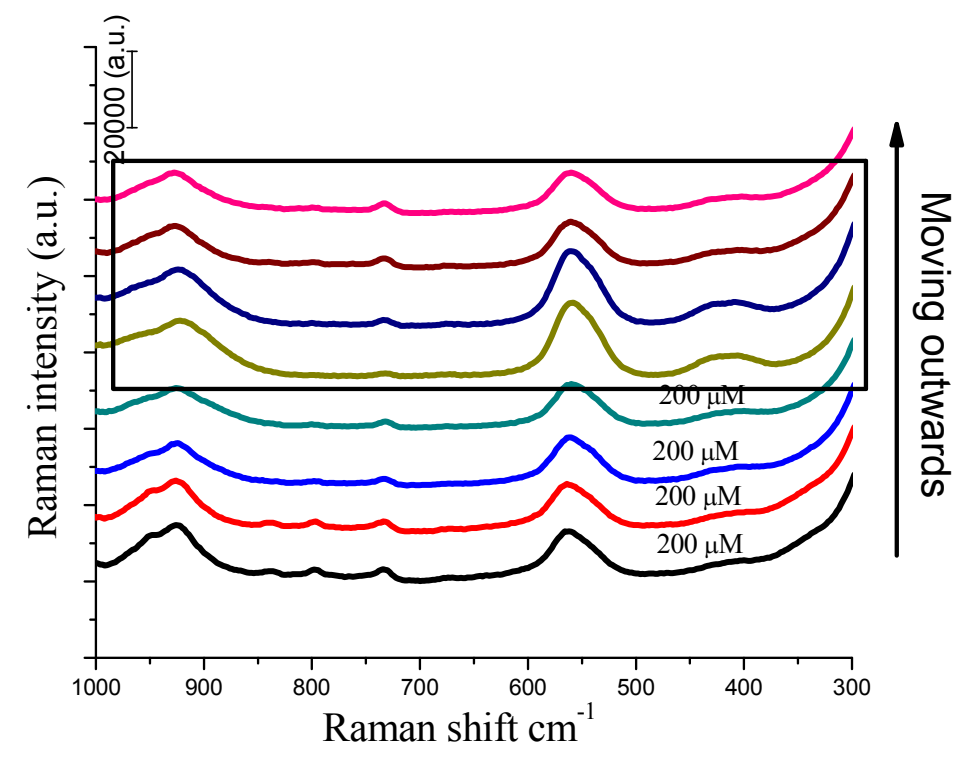

Figure 3.8 SERS signals along the radius of the sessile droplet containing $0.05 \%$ CTAB.

On the other hand, SDS was compatible with these three buffer systems and had no interference with arsenic species sensing, especially in the range of $1000 \sim 300 \mathrm{~cm}^{-1}$ (Fig. 3.4). Halo coffee ring was observed in these three buffer systems with the addition of $0.05 \%$ SDS, and an example using phosphate buffer was shown in Fig. 3.7. This unique halo coffee ring phenomenon featured the following characteristics, including the pinned contact line before the complete evaporation of the sessile droplet (same as the typical coffee ring phenomenon) and the outwards flow driven by capillary action. After the sessile droplet was completely evaporated, the signature halo coffee ring was left on the AgNF surface, which had a darker colored inner ring and a lighter colored halo symmetrically. After carefully controlling the buffer composition and the concentrations of surfactants, liquid from the sessile droplet would overcome the constraint from the surface tension and permeate into the peripheral region of the droplet. This concept 
serves as the same principles of circular TLC, but circular TLC typically uses organic solvent as the developing solution. Nano-size AgNPs provided varying retention abilities towards different arsenic compounds during the migration outside the sessile droplet, resulting in different migration distances and thus separation of different arsenicals.

The separation of arsenic species based on the halo coffee ring phenomenon was demonstrated as follows.
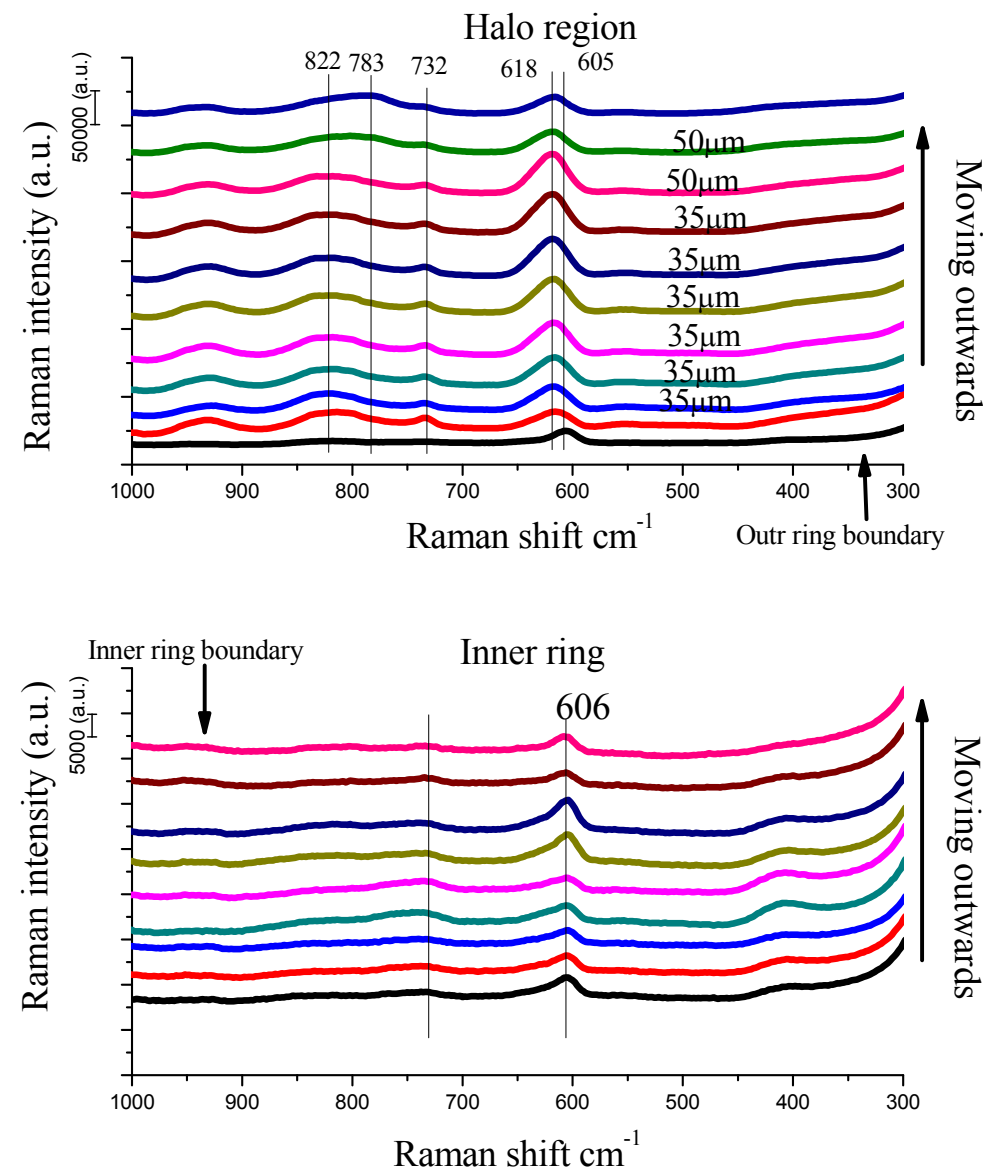

Figure 3.9 Halo coffee ring SERS signal profile of 4 arsenic species in acetic acid. 

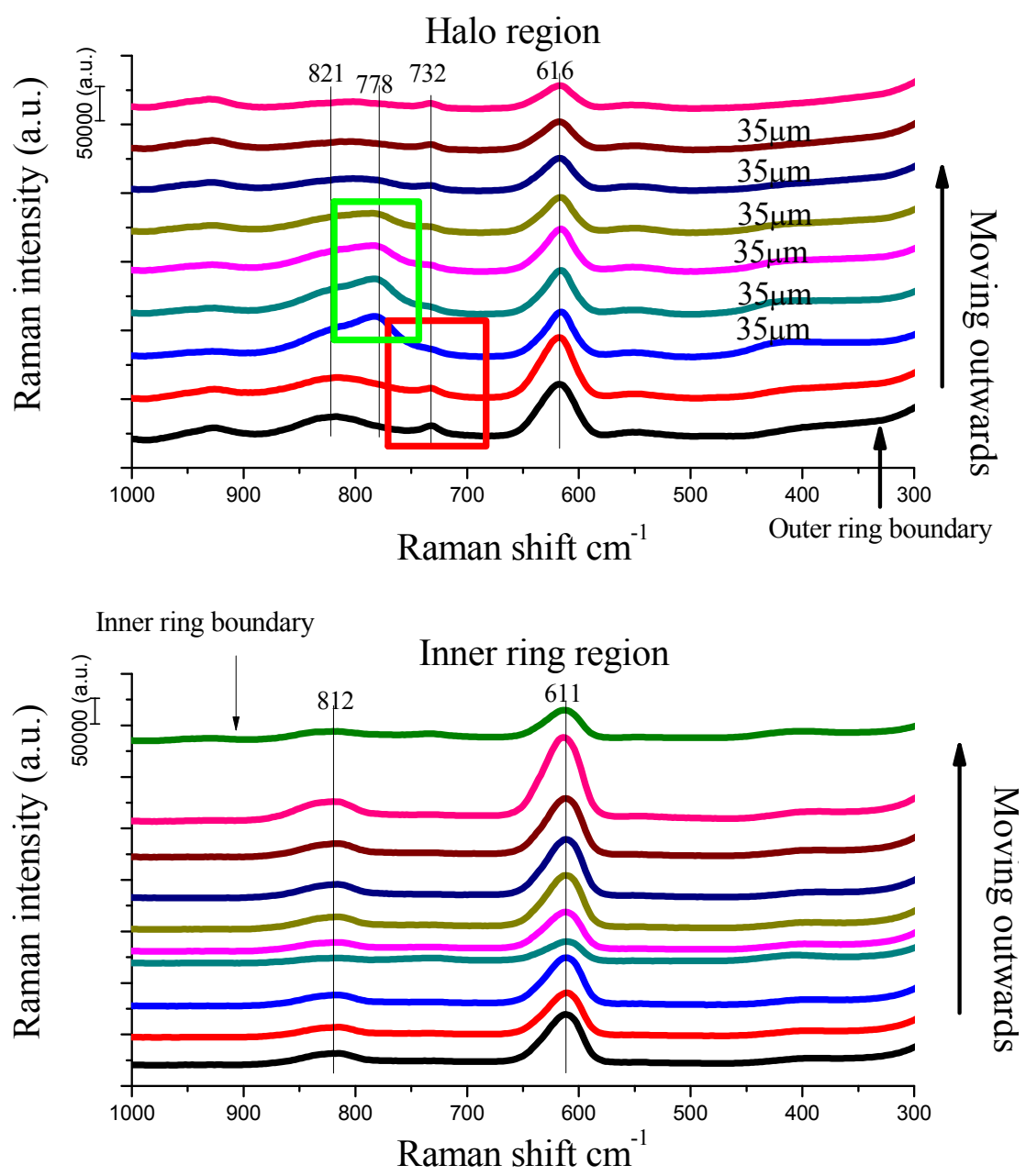

Figure 3.10 Halo coffee ring SERS signal profile of 4 arsenic species in phosphate buffer.

In acetic acid buffer (see Fig. 3.9), only one vibrational band at $606 \mathrm{~cm}^{-1}$ was found in the inner ring area, presumably representing $\mathrm{DMA}^{\mathrm{V}}$. When the sampling spots moved into the halo ring region, significant changes in SERS profiles were found. Firstly, an obvious red-shift of As-C vibrational band was found, which, together with the appearance of $822 \mathrm{~cm}^{-1}$ band (slight blue-shift from original $827 \mathrm{~cm}^{-1}$ obtained from 
$\mathrm{MMA}^{\mathrm{V}}$ coffee ring edge), should indicate that $\mathrm{MMA}^{\mathrm{V}}$ dominated in the halo region. A series of weak signals at $732 \mathrm{~cm}^{-1}$ were found along the halo region, probably indicative of the presence of $\mathrm{As}^{\mathrm{III}}$. At the outmost of the halo region, a $783 \mathrm{~cm}^{-1}$ band was found, which should suggest the presence of $\mathrm{As}^{\mathrm{V}}$. In the $\mathrm{pH}=7.5$ phosphate buffer (see Fig. 3.10), only 611 and $812 \mathrm{~cm}^{-1}$ vibrational bands were found in the inner ring region. Where it moved outwards, the dominant SERS signal was the band at $616 \mathrm{~cm}^{-1}$. With the sampling spots moving outwards, $\mathrm{As}^{\mathrm{III}}$ (the band at $732 \mathrm{~cm}^{-1}$ ) and $\mathrm{As}^{\mathrm{V}}$ (the band at $778 \mathrm{~cm}^{-1}$ ) signals were found subsequently and the $\mathrm{As}^{\mathrm{III}}$ intensity was fairly weak while the $\mathrm{As}{ }^{\mathrm{V}}$ intensity was acceptable. In the ammonium formate buffer (see Fig. 3.11), a similar phenomenon was observed as in previous two buffer systems, in the inner ring region, only one band at $609 \mathrm{~cm}^{-1}$ was found. When it moved outwards, the As-C vibrational band shifted to $619 \mathrm{~cm}^{-1}$. Associated with $619 \mathrm{~cm}^{-1}$ band, there was $\mathrm{s} 819 \mathrm{~cm}^{-1}$ appearing on the spectra, which indicated the existing of MMA ${ }^{\mathrm{V}}$. The band at $730 \mathrm{~cm}^{-1}$ represented $\mathrm{As}^{\mathrm{III}}$ showed up earlier than the one at $780 \mathrm{~cm}^{-1}$, indicating $\mathrm{As}^{\mathrm{V}}$.

Generally, the arsenic separation in these three buffer systems is summarized as the following. Only one arsenic compound was left in the inner ring region, which was $\mathrm{DMA}^{\mathrm{V}}$ despite there was minor difference in As-C vibrational frequencies in these three buffer systems. In the halo ring region, fingerprint signals of $\mathrm{As}^{\mathrm{III}}, \mathrm{As}^{\mathrm{V}}, \mathrm{MMA}^{\mathrm{V}}$ were found with $\mathrm{MMA}^{\mathrm{V}}$ dominated in large area and $\mathrm{As}^{\mathrm{V}}$ migrated further than $\mathrm{As}^{\mathrm{III}}$. Secondly, the signals intensity in the halo region had a universal trend, the signals decreased with the distance heading the halo ring edge, however, this might not be application for $\mathrm{As}^{\mathrm{V}}$ since it appeared on the way to the edge. Thirdly, there was significant SERS signals profiles changing between inner ring and halo ring, usually started at the first sampling 
sport of the halo ring, sometimes even including the outer ring boundary. Fourthly, these two organoarsenicals might not be separated totally in certain buffer systems, such as phosphate buffer (potassium salt) and ammonium formate, because the AsC fingerprint frequencies were deviated from according solo organoarsenicals. In the phosphate buffer (potassium salt), the As-C vibrational frequency were found 611 and $616 \mathrm{~cm}-1$ in inner ring and halo respectively. It was believed in the inner ring region some $\mathrm{MMA}^{\mathrm{V}}$ was not able to migrate into halo ring and elevated the frequency, while some $\mathrm{DMA}^{\mathrm{V}}$ was migrated into the halo ring and reduced the frequency. In the ammonium formate buffer, only some $\mathrm{MMA}^{\mathrm{V}}$ left in the inner ring.
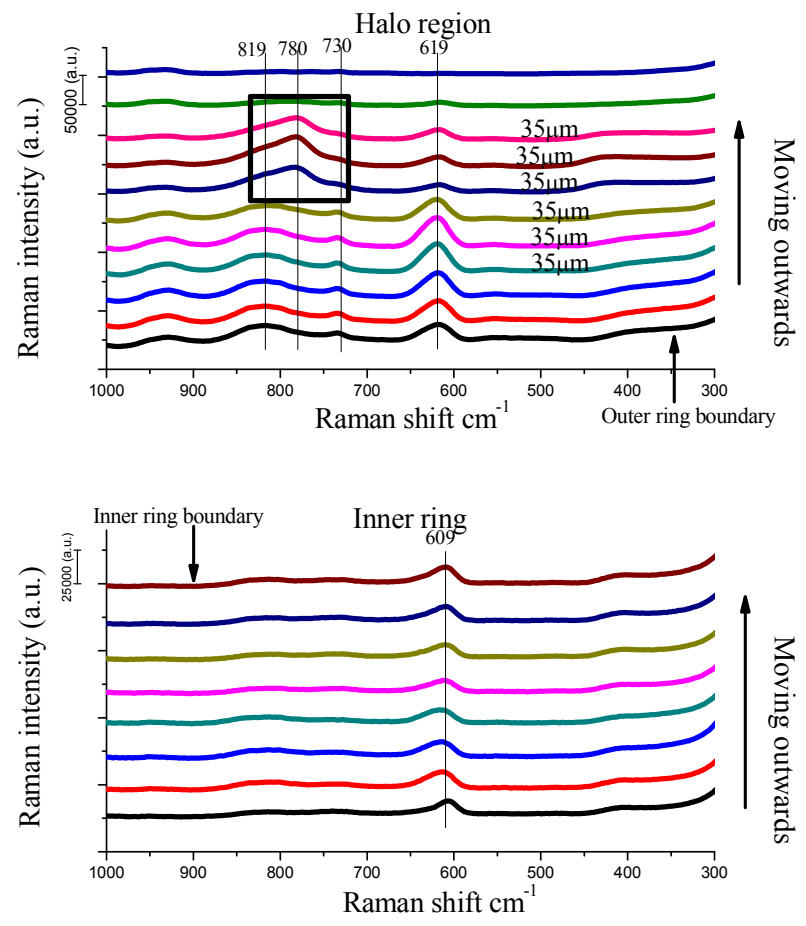

Figure 3.11 Halo coffee ring SERS signal profile of 4 arsenic species in ammonium formate. 
The AgNF surface played a significant role for the arsenic separation, and the charge status was the most critical factor. There was no direct method to determine the surface charge of the AgNF, thus, AgNPs from the surface were washed off using $\mathrm{NaBH} 4$ under sonication and re-suspend into these three buffer systems used respectively [142]. Zeta potential data obtained from these solutions were all negatively charged. Even through the AgNPs were attached to the surface via amine groups on the surface and the amine groups should have an unneglectable effect on the AgNF surface charge. However, the potential positively charged resulted from the amine groups on the glass substrate is ignored owing to the following reasons. Firstly, the glass surface was coating with closepack nanoparticles and thus it was believed there is very few free amine groups exposed because they are all binding to the silver nanoparticles. Furthermore, the surface of AgNPs on the AgNF was assumed to be surrounded by buffer solution during the arsenic migration. Therefore, the zeta potential data were used as an alternative for a better understanding of arsenic migration behavior.

$\mathrm{DMA}^{\mathrm{V}}$ was mostly left in the inner ring region, and the possible reason could be partially due to the relatively high $\mathrm{pKa}$ value $(\mathrm{pKa}=6.2)$. It maintained neutral in acetic acid and ammonium formate buffer systems, and as a result it was not migrated into the halo ring region. In the halo ring $\mathrm{As}^{\mathrm{V}}$ was usually found in the foremost area despited the prevalent distribution of $\mathrm{MMA}^{\mathrm{V}}$, the strong deprotonation ability could contribute to this. It was negatively charged under these buffer systems and the surface was also negatively charged. Therefore, there was no significant attraction from the AgNF during its migration outwards and it was pushed to front. For the prevalent distribution of MMAV, the possible reason could be loose binding with the AgNPs on the AgNF. 
In the capillary electrophoresis separation., arsenic species were separated by their deprotonation status in the buffer [77] and $\mathrm{As}^{\mathrm{III}}$ eluted first and $\mathrm{As}^{\mathrm{V}}$ the last. The reason was that $\mathrm{As}^{\mathrm{III}}$ was neutral and $\mathrm{As}^{\mathrm{V}}$ was highly deprotonated in the $\mathrm{NaH}_{2} \mathrm{PO}_{4}-\mathrm{Na}_{2} \mathrm{~B}_{4} \mathrm{O}_{7}$ buffer $(\mathrm{pH}=6.5)$. Similar to the $\mathrm{CE}$ technique, $\mathrm{As}^{\mathrm{V}}$ was eluted the last in the ion chromatography separation due to low pKa value [212]. However, $\mathrm{As}^{\mathrm{V}}$ was barely migrated in TLC using cellulose as the migration plate [213, 214]. It was reasonable since the migration solution was in high $\mathrm{pH}$ and there were lots of hydroxyl grounds existing on the cellulose plate surface, which ensure the active binding of $\mathrm{As}^{\mathrm{V}}$ onto the surface via chelating complexation. In this study, the electrostatic interactions between arsenicals and the AgNPs on the AgNF plays a critical separation role during the migration along the radius from the center to the edge. At the same time there was non-specific binding found for $\mathrm{MMA}^{\mathrm{V}}$, however, the detail reason was not clear.

\subsection{Conclusion}

In this study, arsenic speciation based on the coffee ring effect was explored and it seems promising to achieve the arsenic separation by using the coffee ring effect under the regulation of surfactants. Due to the strong capillary flow generated by the coffee ring effect, arsenicals accumulated at the edge of the coffee ring and the separation was hard to achieve. In the presence of surfactants, a new ring phenomenon-halo coffee ring effect occurred; solution in the droplet was able to migrate into peripheral region. During the migration into the peripheral region, arsenicals had different interactions with the surface and thus partial separation was achieved. This halo coffee ring effect was demonstrated in different solutions, including $0.1 \mathrm{M}$ acetic acid, phosphate buffer (potassium salt) and ammonium formate. Partial separation of common arsenic species was observed in these 


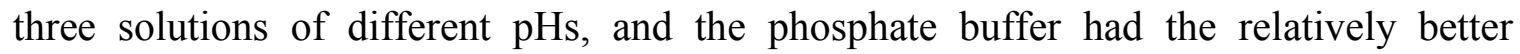
separation effect. Charge status of individual arsenicals played an important role during the migration and eventually caused the separation, since the interaction between arsenicals and the AgNF surface was mostly depending on the electrostatic interaction. However, there are other factors participate in the arsenic migration and separation during the formation of halo coffee ring. 


\section{Chapter 4}

Raman Spectra of Thiolated Arsenicals with Biological Importance 


\subsection{Abstract}

Surface enhanced Raman scattering (SERS) has great potential as an alternative tool for arsenic speciation in biological matrices. The SERS measurements have advantages over other techniques because of its ability to maintain the integrity of arsenic species and its minimal requirements for sample preparation. Up to now, very few Raman spectra of arsenic compounds have been reported. This observation in current literature is particularly true for thiolated arsenicals, which have recently been found to be widely present in humans. The lack of data for Raman spectra in arsenic speciation hampers the development of new tools using SERS. Herein, we report the results of a study combining the analysis of experimental Raman spectra with that obtained from computational calculations for some important arsenic metabolites. The results were obtained with a hybrid functional B3LYP approach using different basis sets to calculate Raman spectra of the selected arsenicals. By comparing experimental and calculated spectra of dimethylarsinic acid $\left(\mathrm{DMA}^{\mathrm{V}}\right)$, the basis set $6-311++\mathrm{G}^{* *}$ was found to provide computational efficiency and precision in vibrational frequency prediction. The Raman frequencies of a set of six organoarsenicals were studied using this basis set, including monomethylarsonous acid $\left(\mathrm{MMA}^{\mathrm{III}}\right)$, dimethylarsinous acid (DMA $\left.{ }^{\mathrm{III}}\right)$, dimethylmonothioarinic acid $\left(\mathrm{DMMTA}^{\mathrm{V}}\right)$, dimethyldithioarsinic acid $\left(\mathrm{DMDTA}^{\mathrm{V}}\right), \mathrm{S}-$ (Dimethylarsenic) cysteine (DMA ${ }^{\mathrm{III}} \mathrm{Cys}$ ) and dimethylarsinous glutathione (DMA $\left.{ }^{\mathrm{III}} \mathrm{GS}\right)$. The results were compared with fingerprint Raman frequencies from $\mathrm{As}-\mathrm{O}, \mathrm{As}-\mathrm{C}$, and As-S obtained under different chemical environments. These fingerprint vibrational frequencies should prove useful in future measurements of different species of arsenic using SERS. 


\section{Key words: Arsenic metabolism • Density functional theory • Raman spectra}

\section{- Thiolated arsenicals • B3LYP}

\subsection{Introduction}

Arsenic as a notoriously toxic pollutant exists widely in the environment. Extensive arsenic contamination of groundwater has led to widespread poisoning in many regions [30-33]. On the other hand, certain arsenic compounds have been used as medicines for treatment of cancers such as acute promyelocytic leukemia, and lymphoma $[215,216]$. Chronic exposure or direct ingestion of arsenicals can result in extensive metabolism and transformation in biological systems, resulting in a wide variety of arsenicals with drastically different chemical properties and toxicities [217-219]. Methylated arsenicals including monomehtylarsonic acid $\left(\mathrm{MMA}^{\mathrm{V}}\right)$, dimethylarsinic acid $\left(\mathrm{DMA}^{\mathrm{V}}\right)$, monomethylarsonous acid $\left(\mathrm{MMA}^{\mathrm{III}}\right)$, and dimethylarsinous acid $\left(\mathrm{DMA}^{\mathrm{III}}\right)$ are generally believed to be the major arsenic metabolites [220-222]. However, thiolated arsenicals have been recently identified in various biological samples. Dimethylarsinothioyl glutathione complex $\left(\mathrm{DMMTA}^{\mathrm{V}}(\mathrm{GS})\right)$ was first detected in cabbages following exposure to $\mathrm{DMA}^{\mathrm{V}}$ [223] and also identified in human cell lines after darinaparsin (S-dimethylarsino-glutathione) exposure $\quad\left[\begin{array}{lll}183, & 224, & 225]\end{array}\right.$ Monomethylmonothioarsonic acid $\left(\mathrm{MMMTA}^{\mathrm{V}}\right)$ was found in urine of hamsters, rats, human [226-229], and in human gut microbiota [230]. Dimethylmonothioarinic acid $\left(\right.$ DMMTA $\left.^{\mathrm{V}}\right)$ was also detected in many biological samples [227, 229, 231-235], such as urine, liver and kidney homogenates, plasma, and red blood cells. It is now recognized that more arsenic metabolites may exist in human than previously believed. Identification 
of arsenic species in different biological matrices is of great importance in the study and assessment of metabolites of arsenic in vivo.

Generally, speciation of arsenic has been carried out by a combination of chromatographic separation and spectroscopic detection. High-performance liquid chromatography (HPLC) [176, 183, 224, 225], ion chromatography (IC) [78, 79] and capillary electrophoresis (CE) [236-238] have been popular separation techniques for these species. Following chromatographic separation, inductively coupled plasma mass spectrometry (ICP-MS) [176, 183, 224, 225], electrospray ionization mass spectrometry (ESI-MS) [72], and time-of-flight mass spectrometry (TOF-MS) [81] have been used for detection, of which ICP-MS and ESI-MS are most widely used. Before analysis can be conducted, extraction of specific arsenicals from different sample matrices usually has to be performed. However, extraction steps employing acid/base or organic solvents may cause alterations in arsenic speciation. For example, it was reported in one sample preparation method that only $55 \%$ of $\mathrm{DMA}^{\mathrm{III}}$ remained following extraction and cleanup [239]. Speciation analysis of arsenic in biological samples remains a challenging task because of the diversity of arsenic metabolites and their instability. Therefore the development of a method for arsenic speciation that does not require extensive sample preparation would be ideal. Raman spectroscopy provides molecular fingerprint information and should be a good choice for elemental speciation analysis. Raman spectroscopy provides great structural information using vibrational spectra, and unlike infrared methods, water is not an interferent [100], which makes Raman spectroscopy readily adapted to in vivo studies [240, 241]. Surface-enhanced Raman spectroscopy (SERS) employs metallic nanostructured substrates to improve the signal sensitivity 
using localized surface plasmon resonant field enhancements. In recent years, the use of SERS in bioanalytical fields has increased exponentially as a result of advances in the preparation of nanomaterials [242-245]. It is potentially possible to employ SERS for arsenic speciation analysis in vivo. However, reports related to the application of SERS in this area are limited because of the lack of reported Raman spectra and vibrational assignments for most arsenic metabolites. In previous work, the Raman spectra of mixtures of arsenic and sulfur vapors were investigated using high-temperature laser. Complicated interactions between arsenic and sulfur were observed various Raman vibrational bands as shown on the Raman spectra. Unfortunately, potential As-S vibrational movements were not assigned in the paper [246]. The Raman spectra of $\mathrm{Na}_{3} \mathrm{AsS}_{4} \cdot 8(\mathrm{D}, \mathrm{H})_{2} \mathrm{O}$ and $\mathrm{Na}_{3} \mathrm{SbS}_{4} \cdot 9(\mathrm{D}, \mathrm{H})_{2} \mathrm{O}$ were measured in salt hydrates, providing valuable information of As-S bonds despite lacking spectrum unfolding [247]. However, with the discovery of new arsenic metabolites, particularly the thiolated organoarsenicals, the information on the Raman spectra of these arsenic compounds is very much needed.

Quantum chemical calculation is an essential method for interpretation of experimental vibrational spectra [248]. Previously, ab initio Hartree-Fock molecular orbital theory was employed to interpret EXAFS and Raman spectral properties of various inorganic sulfur-containing arsenicals [249]. Compared with Hartree-Fock calculations, density functional theory (DFT) including the Becke, 3-parameter, LeeYang-Parr (B3LYP) method [162-165], has been demonstrated to yield good performance when used to predict IR and Raman spectra. The Raman spectra of the As(HCys $)_{3}$ complex was calculated using the DFT method and was compared with Raman frequencies for this same complex observed experimentally. The calculated As-S 
frequencies for $\mathrm{As}(\mathrm{Hcys})_{3}$ were approximately $368-402 \mathrm{~cm}^{-1}$, which provides the important structural information as well as the mechanism of arsenite complexation at a molecular level [250]. The As-O, As-OH, As-S, and As- $\mathrm{SH}$ vibrational frequencies for some inorganic oxoarsenicals and thioarsenicals have also been calculated using the CBSB7 B3LYP method [251]. Nevertheless, many organic arsenic metabolites and thiolated arsenicals still lack spectral analysis. Previously, most theoretical computations were calculated roughly by low-precision methods [249] and focused mainly on inorganic arsenic compounds [251]. The recent discovery of an extensive variety of arsenic metabolites including new thioarsenicals has resulted in an urgent need for these compounds to be characterized by computational and experimental methods. Raman spectroscopy and especially SERS has great potential in the study of arsenic metabolism, but there is currently a lack of data for these studies.

The objective of the present work was to study the Raman spectra of some key organoarsenicals that have been identified in biological samples or could potentially be important in arsenic metabolism by comparison of computational and experimental results. The selected arsenicals included thiolated arsenicals which were either purchased or synthesized in the laboratory. Raman spectra of the selected compounds were measured experimentally with protection from air oxidation for certain oxygen sensitive species $\left(\mathrm{MMA}^{\mathrm{III}}, \mathrm{DMA}^{\mathrm{III}}, \mathrm{DMMTA}^{\mathrm{V}}\right.$, DMDTA ${ }^{\mathrm{V}}, \mathrm{DMA}^{\mathrm{III}} \mathrm{Cys}$, DMA ${ }^{\mathrm{III}} \mathrm{GS}$. Quantum chemistry methods were employed to simulate molecular vibrations associated with the Raman spectra in order to assist in the assignment of vibrational bands of the arsenicals. Overall this research provides important information on Raman spectra for some 
potentially important arsenic metabolites and sheds light on future analysis of arsenic metabolites in vivo using SERS.

\subsection{Materials and Chemicals}

Cacodylic acid sodium salt, 98\% (DMA ${ }^{\mathrm{V}}$-Salt) and cacodylic acid, 98\% $\left(\mathrm{DMA}^{\mathrm{V}}\right)$ were purchased from Sigma-Aldrich, USA. Monosodium acid methane arsonate sesquihydrate, $98 \%\left(\mathrm{MMA}^{\mathrm{V}}\right)$ was purchased from Chem Service, USA. L-Cysteine (cell culture reagent) was purchased from Acros Organics, USA. L-Cystine (Sigma grade) was purchased from Sigma-Aldrich, USA. $\mathrm{NaOH}, \mathrm{HCl}, \mathrm{H}_{2} \mathrm{SO}_{4}, \mathrm{P}_{2} \mathrm{O}_{5}, \mathrm{KI}, \mathrm{Na}_{2} \mathrm{~S} \bullet 9 \mathrm{H}_{2} \mathrm{O}$ were certified ACS grade and purchased from Fisher Scientific, USA. Sulfur dioxide $\left(\mathrm{SO}_{2}\right)$ gas and nitrogen $\left(\mathrm{N}_{2}\right)$ gas were purchased from Specialty Gases of America, USA and Airgas, USA, respectively. Anhydrous ethyl alcohol (ACS/USP grade) was purchased from Pharmco-Aaper, USA. Ethyl ether anhydrous (ACS certified) was purchased from Fisher Scientific, USA. DI water (18.2 M 2 ) was produced in the laboratory using a Barnstead Nanopure Diamond, USA.

\subsection{Experimental procedures}

\subsubsection{Synthesis of arsenicals in the laboratory}

$$
\text { 4.4.1.1 } \mathrm{MMA}^{\mathrm{III}} \text { and } \mathrm{DMA}^{\mathrm{III}}
$$

The $\mathrm{MMA}^{\mathrm{III}}$ and $\mathrm{DMA}^{\mathrm{III}}$ were prepared from their corresponding iodine forms according to the procedures reported previously [176]. Briefly, $\mathrm{MMA}^{\mathrm{V}}$ or $\mathrm{DMA}^{\mathrm{V}}$ was mixed with $\mathrm{KI}$ in $\mathrm{DI}$ water, and then concentrated $\mathrm{HCl}$ was added to the solution. The solution was placed on a magnetic stirring plate and a flow of $\mathrm{SO}_{2}$ was used for reduction of the arsenic compound. For $\mathrm{MMA}^{\mathrm{III}} \mathrm{I}_{2}$, a yellow precipitate was formed in the aqueous solution as reduction proceded. The yellow precipitate was collected and dissolved in 
diethyl ether and dried under a stream of $\mathrm{N}_{2}$ gas. For DMA ${ }^{\mathrm{III}} \mathrm{I}$, a yellow oil product was found at bottom of the reaction vial at the end of the reaction. The yellow oil was collected and dried over $\mathrm{P}_{2} \mathrm{O}_{5}$. The drying in $\mathrm{P}_{2} \mathrm{O}_{5}$ must be performed cautiously since heat is released when $\mathrm{P}_{2} \mathrm{O}_{5}$ reacts with water. The dry yellow oil was extracted using diethyl ether and dried under a stream of $\mathrm{N}_{2}$ gas. Saturated solutions of $\mathrm{MMA}^{\mathrm{III}} \mathrm{I}_{2}$ and DMA ${ }^{\mathrm{III}} \mathrm{I}$ were prepared and hydrolyzed in $0.1 \mathrm{M} \mathrm{NaOH}$ prior to Raman spectral measurement.

\subsubsection{DMMTA $^{\mathrm{V}}$ and DMDTA ${ }^{\mathrm{V}}$}

The anhydride form of DMMTA ${ }^{\mathrm{V}}$ was prepared by dissolving $\mathrm{DMA}^{\mathrm{V}}(6.52 \mathrm{~g}$ or $47.2 \mathrm{mmol})$ in $70 \mathrm{ml}$ of $30 \%(\mathrm{wt} / \mathrm{wt})$ aqueous ethanol [252]. Dilute $\mathrm{H}_{2} \mathrm{SO}_{4}(2 \mathrm{M})$ solution was added dropwise onto $\mathrm{Na}_{2} \mathrm{~S} \cdot 9 \mathrm{H}_{2} \mathrm{O}$ crystals to produce $\mathrm{H}_{2} \mathrm{~S}$ in a gas generator and the gas was bubbled into the $70 \mathrm{ml}$ of $\mathrm{DMA}^{\mathrm{V}}$ solution prepared upon for 15 minutes under stirring without heating. After reaction, the solution was dried using a rotary evaporator and the resultant sample was hydrolyzed in degassed DI water. The hydrolysis product was dried again in a rotary evaporator and the dried powder was collected for further analysis.

Dimethyldithioarsinic acid $\left(\mathrm{DMDTA}^{\mathrm{V}}\right)$ was prepared from sodium dimethyldithioarsinic salt (Sodium-DMDTA) [253]. Sodium-DMDTA was prepared by passing hydrogen sulfide $\left(\mathrm{H}_{2} \mathrm{~S}\right)$ through a boiling ethanol solution of dimethylarsinic (DMA ${ }^{\mathrm{V}}$-Salt, $0.584 \mathrm{~mol} / \mathrm{L}, 25 \mathrm{ml}$ ) for half hour. The $\mathrm{H}_{2} \mathrm{~S}$ gas was generated by dropping diluted $\mathrm{H}_{2} \mathrm{SO}_{4}$ into $\mathrm{Na}_{2} \mathrm{~S} \cdot 9 \mathrm{H}_{2} \mathrm{O}$ crystals and the gas was collected and bubbled into the solution using a gas generator. After the reaction flask cooled down, a large amount of precipitate was found at bottom of the flask. Cold ethanol was used to wash the 
precipitate three times and then the precipitate was dried under a stream of $\mathrm{N}_{2}$ gas. The precipitate (sodium salt form) was dissolved in hot ethanol and $2 \mathrm{M} \mathrm{HCl}$ solution was introduced drop by drop in order to yield the product $\operatorname{DMDTA}^{\mathrm{V}}$ (acid form) and byproduct $\mathrm{NaCl}$ as precipitate. Because $\mathrm{NaCl}$ had relatively low solubility in the ethanol solution and it was easily removed by decanting and the organic phase was dried under a stream of $\mathrm{N}_{2}$ gas.

\subsubsection{3 $\quad \mathrm{DMA}^{\mathrm{III}} \mathrm{Cys}$}

The S-(Dimethylarsenic) cysteine $\left(\mathrm{DMA}^{\mathrm{III}} \mathrm{Cys}\right)$ complex was prepared by reduction using a mixture of $\mathrm{DMA}^{\mathrm{V}}(0.66 \mathrm{mmol})$ with L-cysteine $(0.33 \mathrm{mmol})$ in $10 \mathrm{ml}$ of degassed DI water under nitrogen. Our synthesis of DMA ${ }^{\mathrm{III}} \mathrm{Cys}$ used a larger quantity of L-cysteine than DMA ${ }^{\mathrm{V}}$ than described in previous reports $[235,254]$, however, it was found that the cystine byproduct in the precipitate affects the DMA ${ }^{\mathrm{III}} \mathrm{Cys}$ Raman spectra (See Figure S5A). Because DMA ${ }^{\mathrm{III}}$ Cys hydrolyzed and turned into DMA ${ }^{\mathrm{III}}$ during chromatographic separation and ICP-MS detection of ${ }^{75} \mathrm{As}$ in these two previous reports, the co-existing byproduct cystine had no influence on their study. Therefore, a two-fold molar of $\mathrm{DMA}^{\mathrm{V}}$ against L-cysteine was employed to reduce the formation of byproduct - cystine. The reaction was carried out at room temperature using magnetic stirring for three hours [225]. After the reaction, the solution was centrifuged and the supernatant was collected. Then the supernatant was evaporated, the white residue powder was carefully collected for further analysis. 


\subsubsection{Instrumentation}

4.4.2.1 High performance liquid chromatography coupling inductively coupled plasma mass spectrometry (HPLC/ICP-MS) for arsenic speciation

The high-performance liquid chromatography (HPLC) (PerkinElmer series 2000) using a $\mathrm{C} 18$ reversed-phase column and a mobile phase consisting of $3 \mathrm{mM}$ malonic acid, $5 \mathrm{mM}$ tetrabutylammonium hydroxide, and 5\% (v/v) methanol $(\mathrm{pH}=5.9)$ with a flow rate of $1 \mathrm{~mL} / \mathrm{min}$. Arsenic content was detected by an ELAN DRC-e inductively coupled plasma mass spectrometry (ICP-MS) (PerkinElmer). The concentrations of all standard arsenic standards were $1 \mu \mathrm{M}$, including $\mathrm{As}^{\mathrm{III}}, \mathrm{MMA}^{\mathrm{III}}, \mathrm{DMA}^{\mathrm{V}}$ and $\mathrm{MMA}^{\mathrm{V}}$.

\subsubsection{Mass spectrometers}

A. Bruker fourier transform ion cyclotron resonance mass spectrometry (FTICR-MS)

The DMMTA ${ }^{\mathrm{V}}$ sample was analyzed on a Bruker Solarix FT-ICR-MS equipped with a $7 \mathrm{~T}$ superconducting magnet in the Advanced Mass Spectrometry Facility at Florida International University (Florida, USA). The samples were infused using a Bruker API electrospray source at $6 \mu \mathrm{L} / \mathrm{min}$ by a syringe pump. The instrument parameters were set at $4.6 \mathrm{kV}$ for the capillary column front end voltage, and $200 \mathrm{~V}$ capillary end voltage. Ions accumulated for $0.5 \mathrm{~s}$ in hexapole with $6.0 \mathrm{~V}$ direct-current voltage and $180 \mathrm{Vp}-\mathrm{p}$ ratio frequency (RF) amplitude. The optimized mass for quadrupole 1 was set to $100.0 \mathrm{Da}$. Hexapoles of the Qh interface were operated at $5 \mathrm{MHz}$ and $300 \mathrm{Vp}-\mathrm{p}$ amplitude, in which ions accumulated for $0.1 \mathrm{~s}$. Ions were transferred to an ICR cell by electrostatic focusing of the transfer optics. The ICR was operated at 100.4- 
4000.0 mass range, and $4 \mathrm{MW}$ data size. The time domain data sets were co-added from 50 data acquisitions.

\section{B. Thermo Finnigan LCQ Deca XP MAX LC-MS/MS mass spectrometer}

The DMDTA ${ }^{\mathrm{V}}$ powder sample was dissolved in $1 \mathrm{~mL}$ methanol $(50.0 \mathrm{mg} / \mathrm{mL})$ and diluted ten times in $0.1 \%$ ammonium hydroxide in acetonitrile. The sample was analyzed by directed infusion negative ESI mode on the Thermo Finnigan LCQ Deca XP MAX mass spectrometer, and the full scan from 100-1000 Da and the product ion scan from 50-175 Da modes were performed.

\section{QTRAP 5500 LC-MS/MS mass spectrometer}

The DMA ${ }^{\mathrm{III}}$ Cys powder sample was dissolved in $0.3 \mathrm{~mL}$ of $66 \% \mathrm{ACN}$ and $34 \%$ DI water. An aliquot $(50 \mu \mathrm{L})$ of the obtained solution was transferred to a glass vial and mixed with $450 \mu \mathrm{L}$ of ACN. A blank was prepared similarly, substituting the volume of diluted sample with $34 \%$ water in ACN. Using a syringe pump ( $5 \mu \mathrm{L} / \mathrm{min})$, the obtained solutions were combined with the output of an LC system (Thermo Surveyor LC pump), which delivered $50 \mu \mathrm{L} / \mathrm{min}$ of $0.1 \% \mathrm{FA}$ in ACN (Optima LC/MS grade). The combined flow was infused into the Turbo VTM ESI source of an AB SCIEX QTRAP 5500 triple quadrupole mass spectrometer, operated in the positively mode under full scan (100-500 Da) and the product ion scan (50-300 Da) modes.

\subsubsection{Proton NMR spectrometer}

Proton NMR spectra were recorded at $25{ }^{\circ} \mathrm{C}$ on a Bruker 400 FT NMR spectrometer operating at a proton frequency of $400 \mathrm{MHz}$. 


\subsubsection{Raman spectroscopy measurement}

The Raman spectrometer used in this study was a Perkin-Elmer Raman spectroscopy (Raman station 400F), employing a diode laser operating system at $785 \mathrm{~nm}$ with an average power of $100 \mathrm{~mW}$ at the sample and a 100 -micron spot size. This Raman station was equipped with a Perkin-Elmer 300 microscope, which has a movable stage. The laser beam was focused on the sample stage through a fiber optic cable connecting the $400 \mathrm{~F}$ instrument to the microscope. Typically, a 20X optical lens was employed for better sample focusing and response. Raman spectra $\left(3300 \sim 200 \mathrm{~cm}^{-1}\right)$ were recorded using a $785 \mathrm{~nm}$ laser with 1 second of exposure time, and 4 exposures per measurement. Redox-sensitive arsenicals were sealed in melting point capillary tubes to prevent oxidation during measurement, while for stable arsenicals, the Raman spectra were obtained by placing powder samples directly onto microscopy slides. Most arsenical Raman spectra prepared in this study were obtained using this Perkin-Elmer Raman station $400 \mathrm{~F}$.

Another Raman spectrometer used in this work was a Thermo Scientific FirstDefender RM portable handheld Raman spectroscopy. The portable handheld system was equipped with one $785 \mathrm{~nm}$ diode laser and a CCD spectrometer. The Raman shift measurement range for this instrument was $250 \sim 2875 \mathrm{~cm}^{-1}$. It had three levels of output power, low, medium and high, corresponding to 75,125 , and $250 \mathrm{~mW}$, respectively. For Raman spectra obtained from the Thermo Scientific portable handheld Raman spectroscopy, samples in glass vials were directly placed in front of the laser output probe, exposure time was set to 50 seconds and high output power was employed during sample measurement. 


\subsubsection{Theoretical calculation}

The B3LYP functional hybrid software with five different basis sets was employed for arsenic molecular geometry optimization. Subsequently, Raman spectra were calculated at the same level of theory using the optimized molecular structures. Two of Pople's split valence basis sets with different dispersion and polarization levels were first used, including $6-31 \mathrm{G}^{*}$ and $6-311++\mathrm{G}^{* *}$. Considering that arsenic is an element belonging to the fourth period, the scalar relativistic effects might have important influences on the chemical properties of arsenic compounds [255]. The Stuttgart/Dresden double- $\zeta$ (SDD) basis set was employed to describe the arsenic atom in these arsenic compounds, while other atoms were described using $6-31 \mathrm{G}^{*}$ or $6-311++\mathrm{G}^{* *}$ basis sets. The resultant mixed basis sets were denoted as $\mathrm{SDD} / 6-31 \mathrm{G}^{*}$ and $\mathrm{SDD} / 6-311++\mathrm{G}^{* *}$, which were examined to evaluate whether considering relativistic effects would increase the accuracy of computational results. Lastly, a high resource-demanding and all electron basis set of triple- $\zeta$ valence quality, def2-TZVPP [256, 257] was also employed in comparison of basis sets.

All calculations were performed using Gaussian 09 (Revision B.01) running on Florida International University's HPC facility usually using two processors. The MOLDEN program [258] was employed to visualize individual arsenic molecular vibrations at specific vibrational frequencies, while the Multiwfn program was used to convert the Raman activities from Gaussian output files into Raman intensities in order to plot the simulated Raman spectra for arsenicals [259]. The vibrational modes in the DFT Raman spectra were assigned via MOLDEN molecular simulation, while assignments from crystal Raman spectra were employed to verify MOLDEN assignments [187]. 
Raman frequencies of the same vibrational modes from experimental and calculated Raman spectra were extracted for further comparisons. Different empirical scaling factors for Raman frequencies were used in order to reduce the disagreement between calculated and experimental Raman frequencies: 0.9614 for $6-31 \mathrm{G}^{*}$ and $\mathrm{SDD} / 6-31 \mathrm{G}^{*}$ [260], and 0.9679 for $6-311++\mathrm{G}^{* *}$ and SDD/ 6-311++G** [165]. Corrected Raman frequencies were obtained once the scaling factors were applied. However, the empirical scaling factor for the def2-TZVPP basis set was not available. Usually, two sets of errors ratios were calculated, which were between the experimental and calculated Raman frequencies with or without scaling factors. For example, the error ratio between an experimental and a calculated Raman frequency was determined by dividing absolute difference between the calculated and e experimental Raman frequencies by the experimental frequencies accordingly. A root mean square error (RMS) $[165,260]$ was adopted to evaluate the performance of basis sets. 


\subsection{Results}

\subsubsection{Comparison of theoretical and experimental Raman spectra}

The molecular structures of $\mathrm{DMA}^{\mathrm{V}}, \mathrm{DMA}^{\mathrm{III}}, \mathrm{MMA}^{\mathrm{III}}, \mathrm{DMMTA}^{\mathrm{V}}, \mathrm{DMDTA}^{\mathrm{V}}$, $\mathrm{DMA}^{\mathrm{III}} \mathrm{GS}$, and DMA ${ }^{\mathrm{III}} \mathrm{Cys}$ are shown in Fig.4.1. 


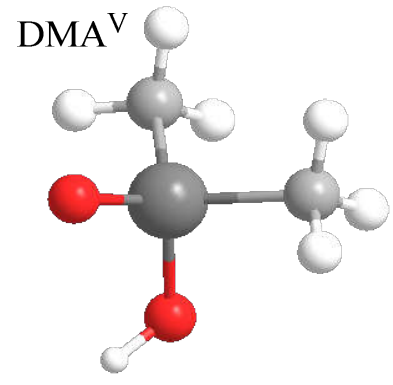

DMMTA $^{\mathrm{V}}$
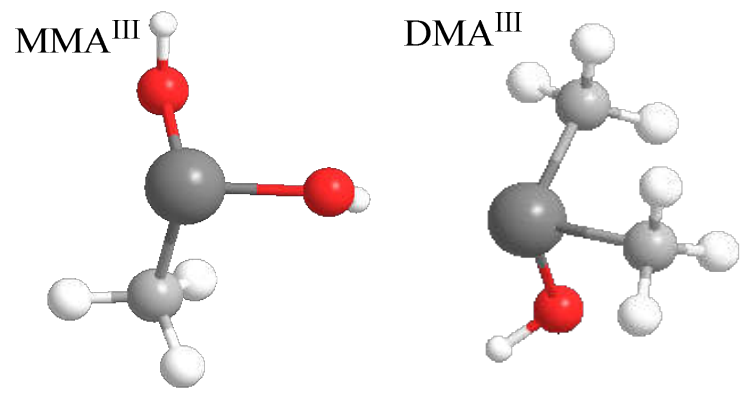

DMDTA $^{\mathrm{V}}$
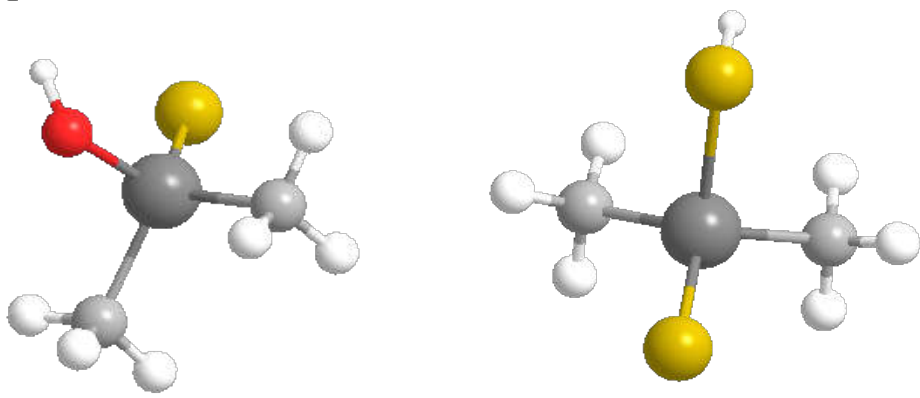

DMA $^{\text {III Cys }}$

DMA $^{\mathrm{III}} \mathrm{GS}$
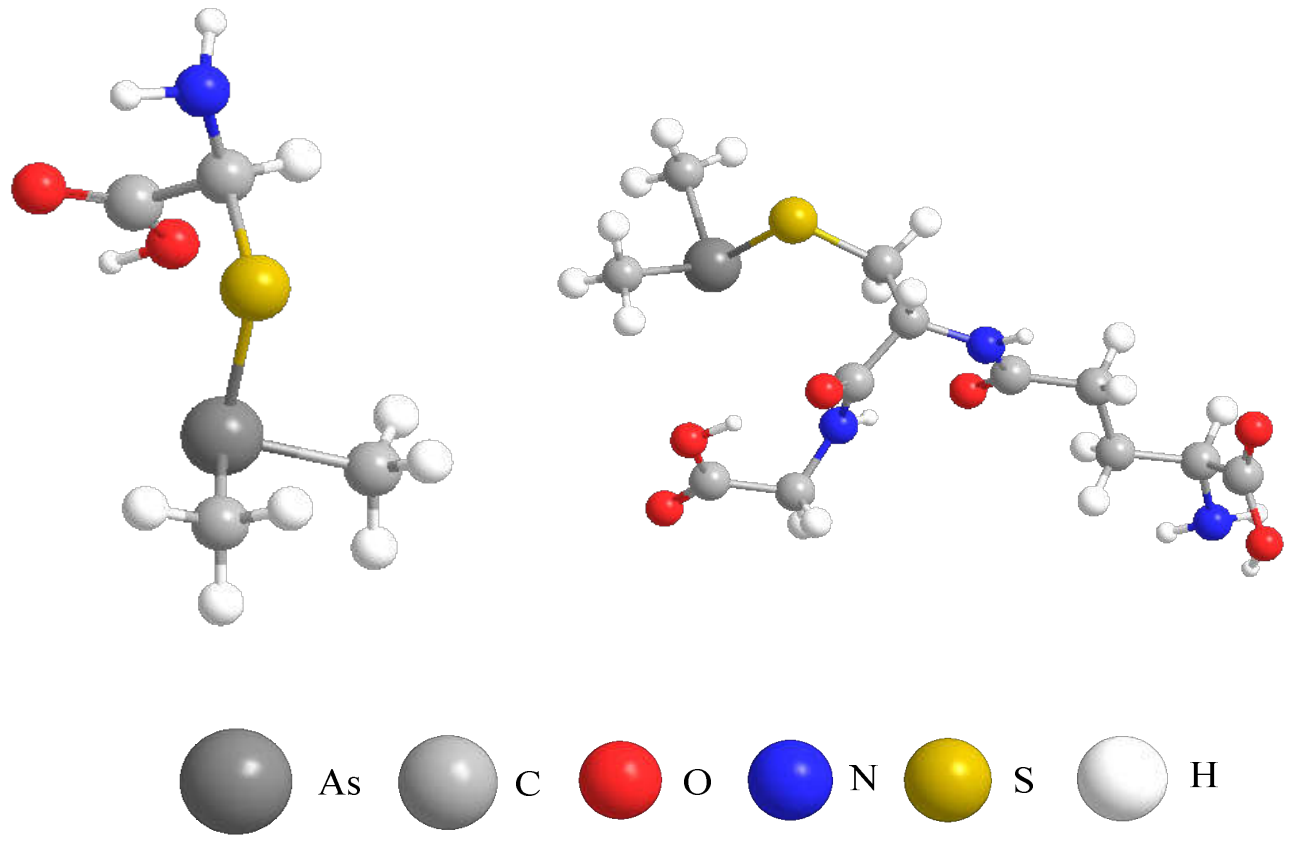

As
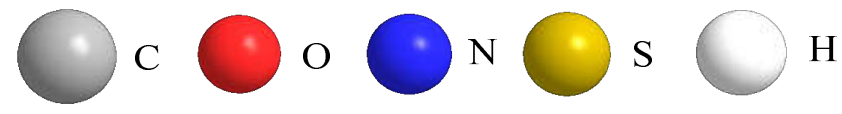

Figure 4.1 3D structures of arsenicals optimized by DFT 
Table 4.1 Comparison of experimental and calculated Raman frequencies for $\mathrm{DMA}^{\mathrm{V}}$ using all basis set

\begin{tabular}{|c|c|c|c|c|c|c|c|c|c|}
\hline$A$ & \multicolumn{3}{|c|}{$6-311++\mathrm{G} * *$} & \multicolumn{3}{|c|}{ No scaling factor } & \multicolumn{3}{|c|}{ With scaling factor } \\
\hline Assignment & $\begin{array}{l}\text { Reported } \\
\text { Raman } \\
\text { frequency } \\
\left(\mathrm{cm}^{-1}\right)\end{array}$ & $\begin{array}{c}\text { Experimental } \\
\text { Raman } \\
\text { frequency } \\
\left(\mathrm{cm}^{-1}\right)\end{array}$ & $\begin{array}{l}\text { Error ratio } \\
\text { between } \\
\text { reported and } \\
\text { experimental } \\
\text { Raman } \\
\text { frequency } \\
(\%)\end{array}$ & $\begin{array}{c}6- \\
311++\mathrm{G}^{* *} \\
\left(\mathrm{~cm}^{-1}\right)\end{array}$ & $\begin{array}{l}\text { Difference } \\
\text { between } \\
\text { experimental } \\
\text { and } \\
\text { theoretical } \\
\text { Raman } \\
\text { frequency } \\
\left(\mathrm{cm}^{-1}\right)\end{array}$ & $\begin{array}{c}\text { Error ratio } \\
(\%)\end{array}$ & $\begin{array}{c}\text { Scaled } \\
\text { frequency } \\
\left(\mathrm{cm}^{-1}\right)\end{array}$ & $\begin{array}{l}\text { Difference } \\
\text { between } \\
\text { experimental } \\
\text { and scalled } \\
\text { theoretical } \\
\text { Raman } \\
\text { frequency } \\
\left(\mathrm{cm}^{-1}\right)\end{array}$ & $\begin{array}{c}\text { Error } \\
\text { ratio } \\
\text { with } \\
\text { scaling } \\
\text { factor } \\
(\%)\end{array}$ \\
\hline $\mathrm{V}_{\mathrm{s}}\left(\mathrm{AsC}_{2}\right)$ & 616 & 611 & 0.8 & 573 & 38 & 6.2 & 555 & 56 & 9.2 \\
\hline $\mathrm{V}_{\mathrm{as}}\left(\mathrm{AsC}_{2}\right)$ & 655 & 636 & 3.1 & 611 & 25 & 3.9 & 591 & 45 & 7.0 \\
\hline $\mathrm{V}(\mathrm{As}-\mathrm{O})$ & 754 & 752 & 0.3 & 634 & 118 & 15.7 & 614 & 138 & 18.4 \\
\hline $\mathrm{V}(\mathrm{As}=\mathrm{O})$ & 822 & 820 & 0.3 & 932 & 112 & 13.7 & 902 & 82 & 10.0 \\
\hline$\delta_{\mathrm{g}}\left(\mathrm{CH}_{3}\right)$ & 1282 & 1279 & 0.5 & 1301 & 22 & 1.7 & 1259 & 20 & 1.5 \\
\hline$\delta_{\mathrm{a}}\left(\mathrm{CH}_{3}\right)$ & 1423 & 1420 & 0.5 & 1453 & 33 & 2.3 & 1406 & 14 & 1.0 \\
\hline $\mathrm{V}_{\mathrm{s}}\left(\mathrm{CH}_{3}\right)$ & 2920 & 2915 & 0.8 & 3054 & 139 & 4.8 & 2956 & 41 & 1.4 \\
\hline $\mathrm{V}_{\mathrm{as}}\left(\mathrm{CH}_{3}\right)$ & 2997 & 2995 & 0.3 & 3148 & 153 & 5.1 & 3047 & 52 & 1.7 \\
\hline $\mathrm{V}(\mathrm{H}-\mathrm{O})$ & NA & NA & NA & 3815 & NA & NA & 3693 & NA & NA \\
\hline
\end{tabular}

\begin{tabular}{|l|l|l|l|}
\hline $\mathrm{B}$ & $6-31 \mathrm{G}^{*}$ & No scaling factor & With scaling factor \\
\hline
\end{tabular}




\begin{tabular}{|c|c|c|c|c|c|c|c|c|c|}
\hline Assignment & $\begin{array}{l}\text { Reported } \\
\text { Raman } \\
\text { frequency } \\
\left(\mathrm{cm}^{-1}\right)\end{array}$ & $\begin{array}{c}\text { Experimental } \\
\text { Raman } \\
\text { frequency } \\
\left(\mathrm{cm}^{-1}\right)\end{array}$ & $\begin{array}{l}\text { Error ratio } \\
\text { between } \\
\text { reported and } \\
\text { experimental } \\
\text { Raman } \\
\text { frequency }(\%)\end{array}$ & $\begin{array}{c}6-31 \mathrm{G}^{*} \\
\left(\mathrm{~cm}^{-1}\right)\end{array}$ & $\begin{array}{l}\text { Difference } \\
\text { between } \\
\text { experimental } \\
\text { and } \\
\text { theoretical } \\
\text { Raman } \\
\text { frequency } \\
\left(\mathrm{cm}^{-1}\right)\end{array}$ & $\begin{array}{c}\text { Error ratio } \\
\quad(\%)\end{array}$ & $\begin{array}{l}\text { Scaled } \\
\text { frequency } \\
\left(\mathrm{cm}^{-1}\right)\end{array}$ & $\begin{array}{l}\text { Difference } \\
\text { between } \\
\text { experimental } \\
\text { and scaled } \\
\text { theoretical } \\
\text { Raman } \\
\text { frequency } \\
\left(\mathrm{cm}^{-1}\right)\end{array}$ & $\begin{array}{l}\text { Error } \\
\text { ratio } \\
\text { with } \\
\text { scaling } \\
\text { factor } \\
(\%)\end{array}$ \\
\hline $\mathrm{V}_{\mathrm{s}}\left(\mathrm{AsC}_{2}\right)$ & 616 & 611 & 0.8 & 578 & 33 & 5.4 & 556 & 55 & 9.1 \\
\hline $\mathrm{V}_{\mathrm{as}}\left(\mathrm{AsC}_{2}\right)$ & 655 & 636 & 3.1 & 611 & 25 & 3.9 & 587 & 49 & 7.6 \\
\hline $\mathrm{V}(\mathrm{As}-\mathrm{O})$ & 754 & 752 & 0.3 & 698 & 54 & 7.2 & 671 & 81 & 10.8 \\
\hline $\mathrm{V}(\mathrm{As}=\mathrm{O})$ & 822 & 820 & 0.3 & 986 & 166 & 20.2 & 948 & 128 & 15.6 \\
\hline$\delta_{\mathrm{g}}\left(\mathrm{CH}_{3}\right)$ & 1282 & 1279 & 0.5 & 1331 & 52 & 4.1 & 1280 & 1 & 0.0 \\
\hline$\delta_{\mathrm{a}}\left(\mathrm{CH}_{3}\right)$ & 1423 & 1420 & 0.5 & 1492 & 72 & 5.1 & 1434 & 14 & 1.0 \\
\hline $\mathrm{V}_{\mathrm{s}}\left(\mathrm{CH}_{3}\right)$ & 2920 & 2915 & 0.8 & 3081 & 166 & 5.7 & 2962 & 47 & 1.6 \\
\hline $\mathrm{V}_{\mathrm{as}}\left(\mathrm{CH}_{3}\right)$ & 2997 & 2995 & 0.3 & 3178 & 183 & 6.1 & 3055 & 60 & 2.0 \\
\hline $\mathrm{V}(\mathrm{H}-\mathrm{O})$ & NA & NA & NA & 3744 & NA & NA & 3599 & NA & NA \\
\hline & \multicolumn{3}{|c|}{$\mathrm{SDD} / 6-31 \mathrm{G}^{*}$} & \multicolumn{3}{|c|}{ No scaling factor } & \multicolumn{3}{|c|}{ With scaling factor } \\
\hline Assignment & $\begin{array}{l}\text { Reported } \\
\text { Raman } \\
\text { frequency } \\
\left(\mathrm{cm}^{-1}\right)\end{array}$ & $\begin{array}{c}\text { Experimental } \\
\text { Raman } \\
\text { frequency } \\
\left(\mathrm{cm}^{-1}\right)\end{array}$ & $\begin{array}{l}\text { Error ratio } \\
\text { between } \\
\text { reported and } \\
\text { experimental } \\
\text { Raman } \\
\text { frequency }(\%)\end{array}$ & $\begin{array}{c}\mathrm{SDD} / \\
6-31 \mathrm{G}^{*} \\
\left(\mathrm{~cm}^{-1}\right)\end{array}$ & $\begin{array}{l}\text { Difference } \\
\text { between } \\
\text { experimental } \\
\text { and } \\
\text { theoretical } \\
\text { Raman } \\
\text { frequency } \\
\left(\mathrm{cm}^{-1}\right)\end{array}$ & $\begin{array}{c}\text { Error ratio } \\
\text { between } \\
\text { experimental } \\
\text { and } \\
\text { theoretical } \\
(\%)\end{array}$ & $\begin{array}{l}\text { Scaled } \\
\text { frequency } \\
\left(\mathrm{cm}^{-1}\right)\end{array}$ & $\begin{array}{l}\text { Difference } \\
\text { between } \\
\text { experimental } \\
\text { and scaled } \\
\text { theoretical } \\
\text { Raman } \\
\text { frequency } \\
\left(\mathrm{cm}^{-1}\right)\end{array}$ & $\begin{array}{l}\text { Error } \\
\text { ratio } \\
\text { with } \\
\text { scaling } \\
\text { factor } \\
(\%)\end{array}$ \\
\hline $\mathrm{V}_{\mathrm{s}}\left(\mathrm{AsC}_{2}\right)$ & 616 & 611 & 0.8 & 569 & 42 & 6.9 & 547 & 64 & 10.5 \\
\hline
\end{tabular}




\begin{tabular}{|c|c|c|c|c|c|c|c|c|c|}
\hline $\mathrm{V}_{\text {as }}\left(\mathrm{AsC}_{2}\right)$ & 655 & 636 & 3.1 & 611 & 25 & 3.9 & 587 & 49 & 7.6 \\
\hline $\mathrm{V}(\mathrm{As}-\mathrm{O})$ & 754 & 752 & 0.3 & 659 & 93 & 12.4 & 634 & 118 & 15.7 \\
\hline $\mathrm{V}(\mathrm{As}=\mathrm{O})$ & 822 & 820 & 0.3 & 932 & 112 & 13.7 & 896 & 76 & 9.3 \\
\hline$\delta_{\mathrm{g}}\left(\mathrm{CH}_{3}\right)$ & 1282 & 1279 & 0.5 & 1342 & 63 & 4.9 & 1290 & 11 & 0.9 \\
\hline$\delta_{\mathrm{a}}\left(\mathrm{CH}_{3}\right)$ & 1423 & 1420 & 0.5 & 1484 & 64 & 4.5 & 1427 & 7 & 0.5 \\
\hline $\mathrm{V}_{\mathrm{s}}\left(\mathrm{CH}_{3}\right)$ & 2920 & 2915 & 0.8 & 3083 & 168 & 5.8 & 2964 & 49 & 1.7 \\
\hline $\mathrm{V}_{\mathrm{as}}\left(\mathrm{CH}_{3}\right)$ & 2997 & 2995 & 0.3 & 3179 & 184 & 6.1 & 3056 & 61 & 2.0 \\
\hline $\mathrm{V}(\mathrm{H}-\mathrm{O})$ & NA & NA & NA & 3737 & NA & NA & 3593 & NA & NA \\
\hline
\end{tabular}

\begin{tabular}{|c|c|c|c|c|c|c|c|c|c|}
\hline \multicolumn{4}{|c|}{$\mathrm{SDD} / 6-311++\mathrm{G} * *$} & \multicolumn{3}{|c|}{ No scaling factor } & \multicolumn{3}{|c|}{ With scaling factor } \\
\hline Assignment & $\begin{array}{l}\text { Reported } \\
\text { Raman } \\
\text { frequency } \\
\qquad\left(\mathrm{cm}^{-1}\right)\end{array}$ & $\begin{array}{c}\text { Experimental } \\
\text { Raman } \\
\text { frequency } \\
\left(\mathrm{cm}^{-1}\right)\end{array}$ & $\begin{array}{l}\text { Error ratio } \\
\text { between } \\
\text { reported and } \\
\text { experimental } \\
\text { Raman } \\
\text { frequency }(\%)\end{array}$ & $\begin{array}{c}\mathrm{SDD} / \\
6- \\
311++\mathrm{G}^{* *} \\
\left(\mathrm{~cm}^{-1}\right)\end{array}$ & $\begin{array}{l}\text { Difference } \\
\text { between } \\
\text { experimental } \\
\text { and } \\
\text { theoretical } \\
\text { Raman } \\
\text { frequency } \\
\left(\mathrm{cm}^{-1}\right)\end{array}$ & $\begin{array}{l}\text { Error ratio } \\
\text { between } \\
\text { experimental } \\
\text { and } \\
\text { theoretical } \\
(\%)\end{array}$ & $\begin{array}{c}\text { Scaled } \\
\text { frequency } \\
\left(\mathrm{cm}^{-1}\right)\end{array}$ & $\begin{array}{l}\text { Difference } \\
\text { between } \\
\text { experimental } \\
\text { and scaled } \\
\text { theoretical } \\
\text { Raman } \\
\text { frequency } \\
\left(\mathrm{cm}^{-1}\right)\end{array}$ & $\begin{array}{c}\text { Error } \\
\text { ratio } \\
\text { with } \\
\text { scaling } \\
\text { factor } \\
(\%)\end{array}$ \\
\hline $\mathrm{V}_{\mathrm{s}}\left(\mathrm{AsC}_{2}\right)$ & 616 & 611 & 0.8 & 559 & 52 & 8.5 & 541 & 70 & 11.4 \\
\hline $\mathrm{V}_{\mathrm{as}}\left(\mathrm{AsC}_{2}\right)$ & 655 & 636 & 3.1 & 606 & 30 & 4.7 & 587 & 49 & 7.8 \\
\hline $\mathrm{V}(\mathrm{As}-\mathrm{O})$ & 754 & 752 & 0.3 & 613 & 139 & 18.5 & 593 & 159 & 21.1 \\
\hline $\mathrm{V}(\mathrm{As}=\mathrm{O})$ & 822 & 820 & 0.3 & 859 & 39 & 4.8 & 831 & 11 & 1.4 \\
\hline$\delta_{\mathrm{g}}\left(\mathrm{CH}_{3}\right)$ & 1282 & 1279 & 0.5 & 1313 & 34 & 2.7 & 1271 & 8 & 0.6 \\
\hline$\delta_{\mathrm{a}}\left(\mathrm{CH}_{3}\right)$ & 1423 & 1420 & 0.5 & 1452 & 32 & 2.3 & 1405 & 15 & 1.0 \\
\hline $\mathrm{V}_{\mathrm{s}}\left(\mathrm{CH}_{3}\right)$ & 2920 & 2915 & 0.8 & 3060 & 145 & 5.0 & 2962 & 47 & 1.6 \\
\hline $\mathrm{V}_{\mathrm{as}}\left(\mathrm{CH}_{3}\right)$ & 2997 & 2995 & 0.3 & 3156 & 161 & 5.4 & 3055 & 60 & 2.0 \\
\hline $\mathrm{V}(\mathrm{H}-\mathrm{O})$ & NA & NA & NA & 3823 & NA & NA & 3700 & NA & NA \\
\hline
\end{tabular}




\begin{tabular}{|c|c|c|c|c|c|c|c|c|c|}
\hline $\mathrm{E}$ & \multicolumn{3}{|c|}{ def2-TZVPP } & \multicolumn{3}{|c|}{ No scaling factor } & \multicolumn{3}{|c|}{ With scaling factor } \\
\hline Assignment & $\begin{array}{l}\text { Reported } \\
\text { Raman } \\
\text { frequency } \\
\left(\mathrm{cm}^{-1}\right)\end{array}$ & $\begin{array}{c}\text { Experimental } \\
\text { Raman } \\
\text { frequency }\end{array}$ & $\begin{array}{l}\text { Error ratio } \\
\text { between } \\
\text { reported and } \\
\text { experimental } \\
\text { Raman } \\
\text { frequency }(\%)\end{array}$ & $\begin{array}{c}\text { def2- } \\
\text { TZVPP } \\
\left(\mathrm{cm}^{-1}\right)\end{array}$ & $\begin{array}{l}\text { Difference } \\
\text { between } \\
\text { experimental } \\
\text { and } \\
\text { theoretical } \\
\text { Raman } \\
\text { frequency } \\
\left(\mathrm{cm}^{-1}\right)\end{array}$ & $\begin{array}{c}\text { Error ratio } \\
\text { between } \\
\text { experimental } \\
\text { and } \\
\text { theoretical } \\
(\%)\end{array}$ & $\begin{array}{c}\text { Scaled } \\
\text { frequency } \\
\left(\mathrm{cm}^{-1}\right)\end{array}$ & $\begin{array}{l}\text { Difference } \\
\text { between } \\
\text { experimental } \\
\text { and scaled } \\
\text { theoretical } \\
\text { Raman } \\
\text { frequency } \\
\left(\mathrm{cm}^{-1}\right)\end{array}$ & $\begin{array}{l}\text { Error } \\
\text { ratio } \\
\text { with } \\
\text { scaling } \\
\text { factor } \\
(\%)\end{array}$ \\
\hline $\mathrm{V}_{\mathrm{s}}\left(\mathrm{AsC}_{2}\right)$ & 616 & 611 & 0.8 & 580 & 31 & 5.1 & \multirow{9}{*}{\multicolumn{3}{|c|}{ NA }} \\
\hline $\mathrm{V}_{\mathrm{as}}\left(\mathrm{AsC}_{2}\right)$ & 655 & 636 & 3.1 & 615 & 21 & 3.3 & & & \\
\hline $\mathrm{V}(\mathrm{As}-\mathrm{O})$ & 754 & 752 & 0.3 & 674 & 78 & 10.4 & & & \\
\hline $\mathrm{V}(\mathrm{As}=\mathrm{O})$ & 822 & 820 & 0.3 & 961 & 141 & 17.2 & & & \\
\hline$\delta_{\mathrm{g}}\left(\mathrm{CH}_{3}\right)$ & 1282 & 1279 & 0.5 & 1298 & 19 & 1.5 & & & \\
\hline$\delta_{\mathrm{a}}\left(\mathrm{CH}_{3}\right)$ & 1423 & 1420 & 0.5 & 1454 & 34 & 2.4 & & & \\
\hline $\mathrm{V}_{\mathrm{s}}\left(\mathrm{CH}_{3}\right)$ & 2920 & 2915 & 0.8 & 2915 & 0 & 0.0 & & & \\
\hline $\mathrm{V}_{\mathrm{as}}\left(\mathrm{CH}_{3}\right)$ & 2997 & 2995 & 0.3 & 2995 & 0 & 0.0 & & & \\
\hline $\mathrm{V}(\mathrm{H}-\mathrm{O})$ & NA & NA & NA & 3811 & NA & NA & & & \\
\hline
\end{tabular}


Table 4.2 Comparisons of computational performance of all basis sets

\begin{tabular}{|c|c|c|c|c|c|c|c|c|c|}
\hline Basis Set & $6-31 G^{*}$ & $\begin{array}{c}\text { Scaled } \\
6-31 G^{*} \\
(0.9614)\end{array}$ & $6-311++\mathrm{G}^{* *}$ & $\begin{array}{c}\text { Scaled } \\
6-311++\mathrm{G}^{* *} \\
(0.9679)\end{array}$ & $\begin{array}{l}\mathrm{SDD} / \\
6-31 \mathrm{G}^{*}\end{array}$ & $\begin{array}{c}\text { Scaled } \\
\text { SDD/ } \\
6-31 G^{*} \\
(0.9614)\end{array}$ & $\begin{array}{c}\mathrm{SDD} / \\
6- \\
311++\mathrm{G}^{* *}\end{array}$ & $\begin{array}{c}\text { Scaled } \\
\text { SDD/6- } \\
311++\mathrm{G}^{* *} \\
(0.9679)\end{array}$ & $\begin{array}{l}\text { def2- } \\
\text { TZVPP }\end{array}$ \\
\hline $\operatorname{RMS}\left(\mathrm{cm}^{-1}\right)$ & 112.4 & 65.6 & 90.9 & 59.4 & 108.2 & 63.8 & 95.9 & 69.5 & 60.1 \\
\hline $\begin{array}{l}\text { Number of error } \\
\text { ratios over } 10 \%\end{array}$ & 1 & 2 & 2 & 1 & 2 & 2 & 1 & 2 & 1 \\
\hline computational cost & $2 \mathrm{~m}, 24 \mathrm{~s}$ & $2 \mathrm{~m}, 24 \mathrm{~s}$ & $11 \mathrm{~m}, 29 \mathrm{~s}$ & $11 \mathrm{~m}, 29 \mathrm{~s}$ & $1 \mathrm{~m}, 37 \mathrm{~s}$ & $11 \mathrm{~m}, 29 \mathrm{~s}$ & $4 m, 58 s$ & $11 \mathrm{~m}, 29 \mathrm{~s}$ & $43 \mathrm{~m}, 5 \mathrm{~s}$ \\
\hline
\end{tabular}


The representative arsenic compound $\mathrm{DMA}^{\mathrm{V}}$ was employed to explore the discrepancy between the DFT Raman spectra, and the experimental Raman spectra using its crystalline Raman spectra [187] as well as to determine which basis set was best for the DFT Raman spectra simulation. The $\mathrm{DMA}^{\mathrm{V}}$ was selected because it is relatively stable and literature results on its Raman frequency were available for comparison. The experimental and calculated Raman frequencies obtained in current study, as well as those reported in the literature for Raman frequencies of $\mathrm{DMA}^{\mathrm{V}}$ [187] along with error ratios using all basis sets are shown in Table 4.1. The employed basis sets were compared and evaluated by the RMS, number of error ratios over $10 \%$ between experimental and calculated Raman frequencies in DMA ${ }^{\mathrm{V}}$, and the computational cost (in term of time consumption) (Table 4.2). It was obvious that using scaling factors greatly reduced the RMS values and improved the precision of the theoretical result, mainly because of the scaling factors being smaller than 1.0. Among all basis sets tested, the basis set $6-311++\mathrm{G}^{* *}$ yielded the smallest RMS values after applying the scaling factor, other basis sets yielded comparable results. On the other hand, it was found that basis sets $6-31 \mathrm{G}^{*}$ and $\mathrm{SDD} / 6-31 \mathrm{G}^{*}$ were not able to correctly predict the intensity of $\mathrm{CH}_{3}$ vibrations from $1600 \sim 1200 \mathrm{~cm}^{-1}$ (Fig. 4.2) as a result of systematic errors, and the basis set def2-TVZPP was not cost effective (Table 4.2). The Raman spectra of DMA ${ }^{\mathrm{V}}$ measured in this study was in good agreement with that previously reported, having an error ratio of the reported vs. the experimental being less than $3.1 \%$ (Table 4.1). The error ratios of the experimentally obtained vs the calculated Raman frequencies of $\mathrm{DMA}^{\mathrm{V}}$ were generally less than $10 \%$, which is acceptable according to previous studies [260262]. However, an over $10 \%$ deviation was observed for the Raman frequencies of As-O and $A s=\mathrm{O}$. The Raman frequencies obtained from experimental measurement and theoretical calculations were 752 and $667 \mathrm{~cm}^{-1}$ for As- $\mathrm{O}$, and 820 and $932 \mathrm{~cm}^{-1}$ for As=O, respectively 
(Table 4.1). The large discrepancy could be attributed to the systematic error occurring during theoretical computation. These types of errors were also found in a series of arsenicals with similar structure (Table 4.3). Furthermore, while application of the scaling factor substantially reduced the RMS, there was a fairly limited impact on error ratios, with the average error ratio being only reduced by 6.1 to $5.8 \%$ following the application of scaling factors (Table 4.2). 

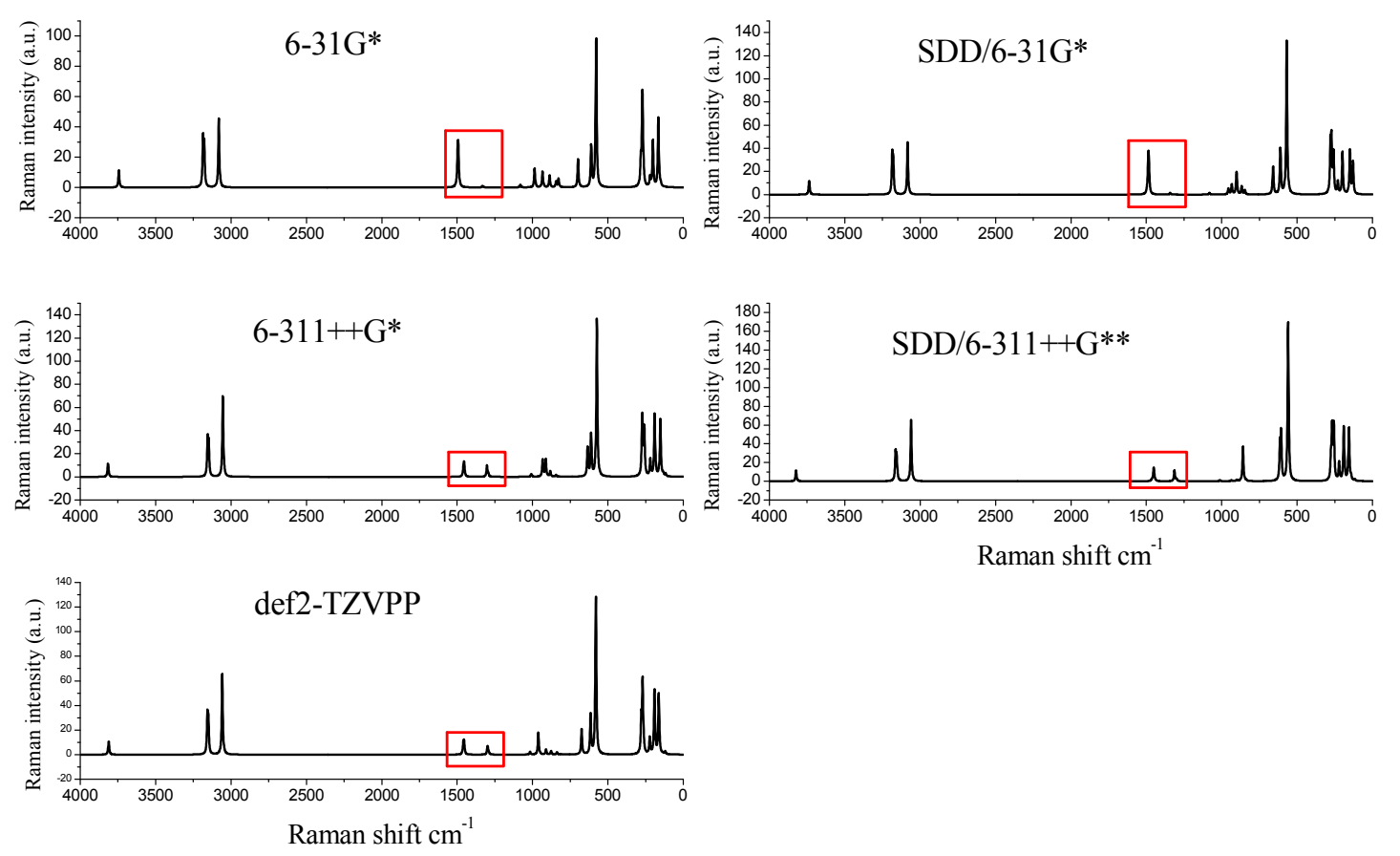

Figure 4.2 Theoretical DMA ${ }^{\mathrm{V}}$ Raman spectra calculated from different basis sets. It was found two basis sets, including 6-31G and $\mathrm{SDD} / 6-31 \mathrm{G}$, had a significant bias in prediction the intensities of bands from 1600 1200 $\mathrm{cm}^{-1}$ (with red frame marks in upon spectra), however, these two bands had almost identical intensity in the Raman spectra. It is the major reason that these two basis sets were not chosen. 
Table 4.3 Comparison of all As-O bonds Raman frequencies between experimental and calculated Raman spectra

\begin{tabular}{|c|c|c|c|}
\hline Arsenical & Assignment & $\begin{array}{c}\text { Experimental } \\
\text { Raman } \\
\text { frequency } \\
\left(\mathrm{cm}^{-1}\right)\end{array}$ & $\begin{array}{c}6- \\
311++\mathrm{G}^{* *} \\
\left(\mathrm{~cm}^{-1}\right)\end{array}$ \\
\hline $\mathrm{As}^{\mathrm{V}}$ & \multirow{3}{*}{$\mathrm{As}=\mathrm{O}$} & 842 & 965 \\
\hline $\mathrm{DMA}^{\mathrm{V}}$ & & 838 & 932 \\
\hline $\mathrm{MMA}^{\mathrm{V}}$ & & 811 & 940 \\
\hline $\mathrm{As}^{\mathrm{V}}$ & $\mathrm{As}-\mathrm{O}$ & 730 & $\begin{array}{c}665 \text { sym } \\
685,705 \\
\text { asym }\end{array}$ \\
\hline DMA $^{\mathrm{V}}$ & $\mathrm{As}-\mathrm{O}$ & 748 & 634 \\
\hline $\mathrm{MMA}^{\mathrm{V}}$ & $\begin{array}{c}\text { As-O(Va, } \\
V s)\end{array}$ & 710 & $\begin{array}{l}661 \text { sym, } \\
664 \text { asym }\end{array}$ \\
\hline
\end{tabular}

Note, sym stands for symmetric stretch, asym stands for asymmetric stretch.

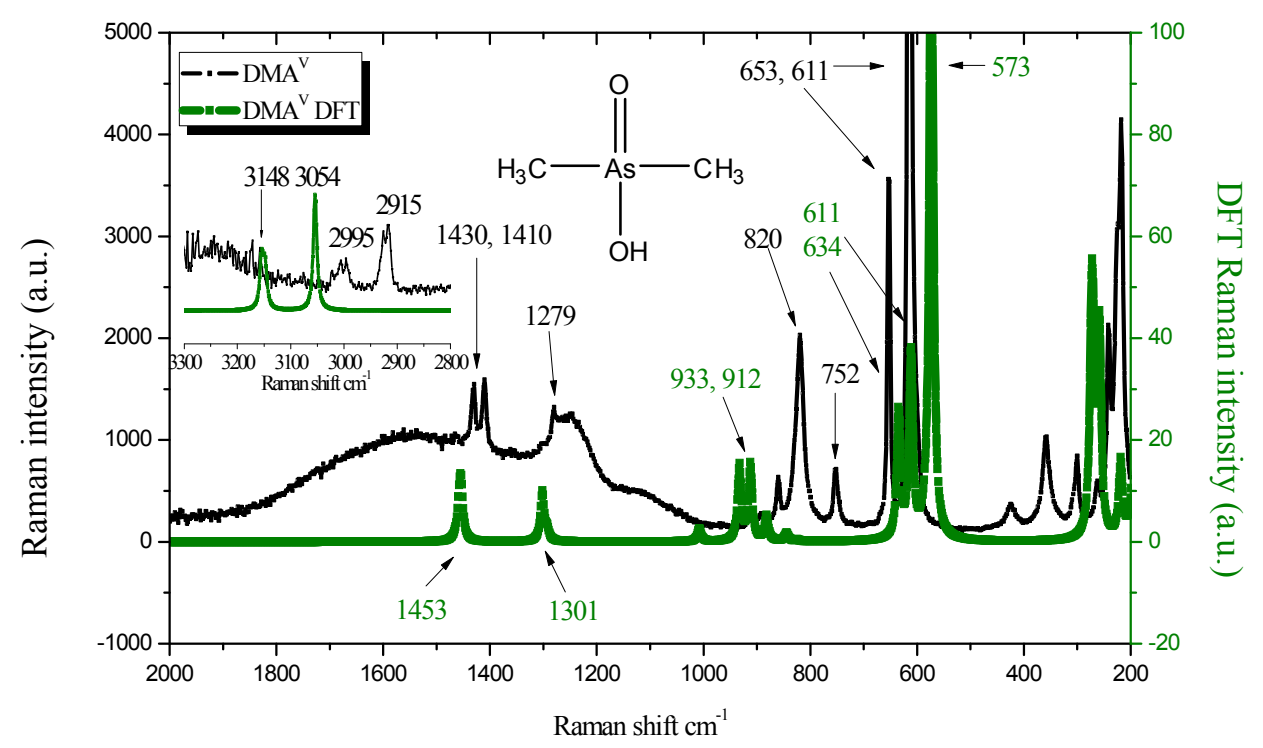

Figure 4.3 Raman spectra of $\mathrm{DMA}^{\mathrm{V}}$ obtained via experimental measurement and calculation

Judging from the number of frequency error ratios over $10 \%$, the medium-sized basis set $6-311++\mathrm{G}^{* *}$ (Table 4.1A) yielded the best results, while the use of mixed basis 
sets that included relativistic effects did not improve the outcome. Although the all electron basis set def2-TZVPP consumed the most computational resources, high demanding basis sets are affordable these days with advances in computational technology. Furthermore, the basis set def2-TZVPP also offers highly comparable RMS results (Table 4.2) without a scaling factor; however, it is difficult to evaluate the results without the scaling factor. Overall, Raman frequencies calculated by the B3LYP with basis set $6-311++\mathrm{G}^{* *}$ gave the optimal result for the identification of $\mathrm{DMA}^{\mathrm{V}}$ Raman spectra. A superimposed Raman spectra of the experimental and the calculated result is shown in Fig. 4.3.

For the Raman vibrational band assignment of the rest of the arsenicals, only following molecular fingerprint vibrational modes were calculated and interpreted in order to characterize arsenicals: As-C stretch (including symmetry and asymmetry), $\mathrm{As}=\mathrm{O}$ stretch, As-O stretch, and the As-S stretch (including single and double bonds). The omission of general molecular vibrational bonds was determined by the following reasons: First, most of these arsenicals were synthesized in the laboratory and used without further purification. Therefore, impurities could generate many unknown vibrational bands, creating interpretational issues. Second, many vibrations in these molecules are weak and difficult to identify. Focusing on these molecular fingerprint vibrational modes will greatly reduce problems with the identification of molecules since these fingerprint vibrations usually yield strong bands for easy identification. Most of the arsenicals studied contain the following bonds, $\mathrm{As}-\mathrm{O}, \mathrm{As}=\mathrm{O}, \mathrm{As}-\mathrm{C}, \mathrm{As}-\mathrm{S}, \mathrm{As}=\mathrm{S}$, thus according to previous studies, the Raman shifts of interest would range from 1000 300 $\mathrm{cm}^{-1}[186-188,263-265]$. Frequencies less than $300 \mathrm{~cm}^{-1}$ were omitted because of the 
complexity of the vibrational modes. Therefore, in the following discussion, only frequencies in the range of $1000 \sim 300 \mathrm{~cm}^{-1}$ are utilized for Raman spectra interpretation.

\subsubsection{Raman spectra interpretation of $\mathrm{MMA}^{\mathrm{III}}$ and $\mathrm{DMA}^{\mathrm{III}}$}

The fingerprint vibrational frequencies and corresponding vibrational mode assignments for MMA ${ }^{\mathrm{III}}$ and DMA ${ }^{\mathrm{III}}$ are summarized in Table 4.4 and their DFT Raman spectra and experimental Raman spectra are illustrated in Fig. 4.4. 

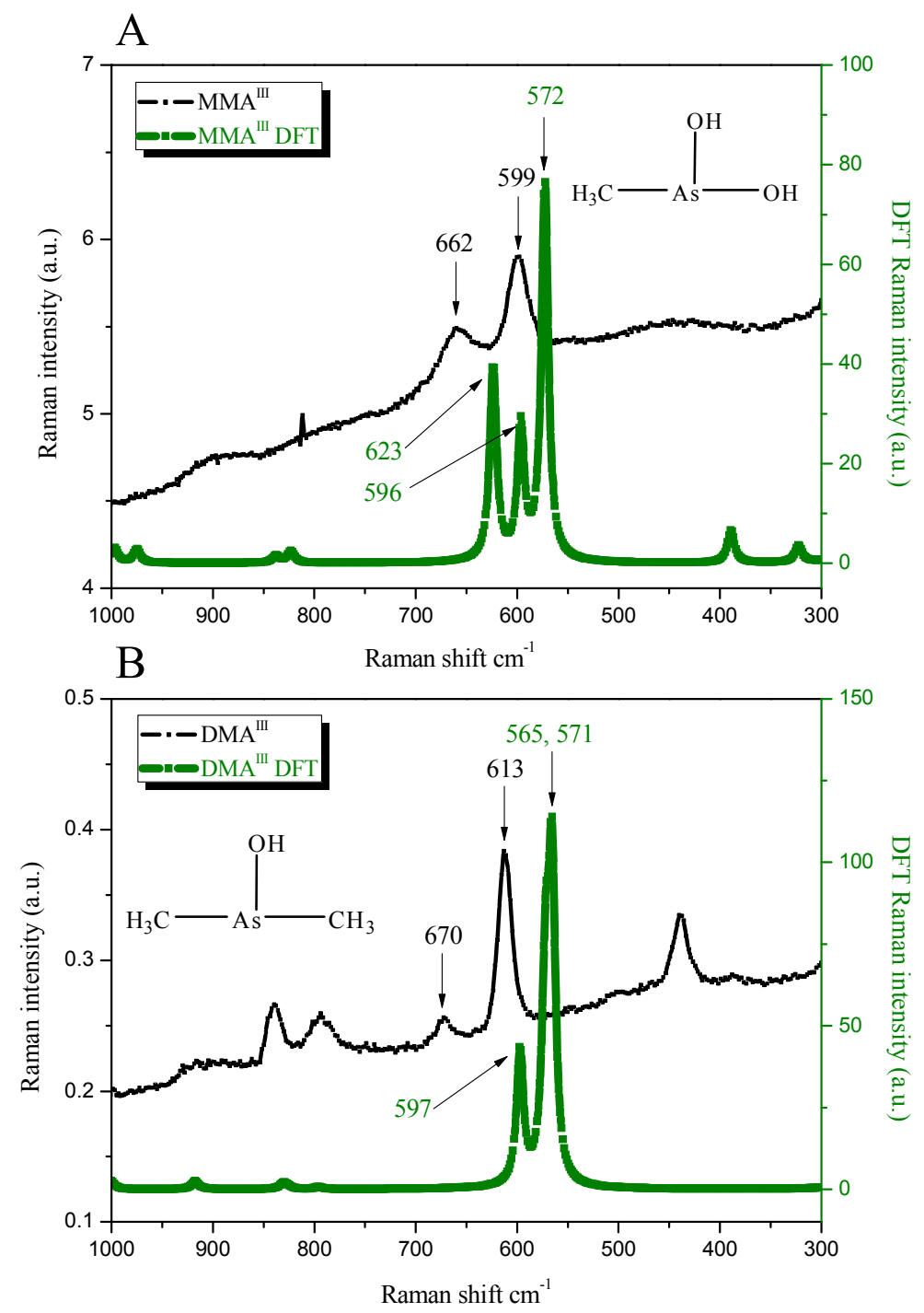

Figure 4.4 Experimental and calculated Raman spectra of $\mathrm{MMA}^{\mathrm{III}}(\mathrm{A})$ and DMA $\mathrm{DII}^{\mathrm{III}}$ (B). (Note Raman spectra was obtained using Thermo FirstDefender RM) 
Table 4.4 Vibrational frequencies assignment for $\mathrm{MMA}^{\mathrm{III}}$ and $\mathrm{DMA}^{\mathrm{III}}$

\begin{tabular}{|c|c|c|c|c|c|c|}
\hline Arsenical & $\begin{array}{l}\text { MOLDEN } \\
\text { assignment }\end{array}$ & $\begin{array}{l}\text { Experimental } \\
\text { Raman } \\
\text { frequency } \\
\left(\mathrm{cm}^{-1}\right)\end{array}$ & $\begin{array}{l}\text { Calculated } \\
\text { Raman } \\
\text { frequency } \\
\left(\mathrm{cm}^{-1}\right)\end{array}$ & $\begin{array}{l}\text { Corrected } \\
\text { Raman } \\
\text { frequency } \\
\left(\mathrm{cm}^{-1}\right)\end{array}$ & $\begin{array}{l}\text { Frequency } \\
\text { difference } \\
\left(\mathrm{cm}^{-1}\right)\end{array}$ & $\begin{array}{l}\text { Error } \\
\text { ratio (\%) }\end{array}$ \\
\hline \multirow{3}{*}{$\mathrm{MMA}^{\mathrm{III}}$} & $\begin{array}{l}\text { As-C } \\
\text { stretch }\end{array}$ & 599 & 572 & 554 & 45.4 & 7.6 \\
\hline & $\begin{array}{l}\mathrm{O}-\mathrm{As}-\mathrm{O} \\
\text { asym stretch }\end{array}$ & NA & 596 & 577 & NA & NA \\
\hline & $\begin{array}{l}\mathrm{O}-\mathrm{As}-\mathrm{O} \\
\text { sym stretch }\end{array}$ & 662 & 623 & 603 & 59.0 & 8.9 \\
\hline \multirow{3}{*}{$\mathrm{DMA}^{\mathrm{III}}$} & $\begin{array}{l}\mathrm{C}-\mathrm{As}-\mathrm{C} \\
\text { sym stretch }\end{array}$ & 613 & 565 & 547 & 66.1 & 10.8 \\
\hline & $\begin{array}{l}\mathrm{C}-\mathrm{As}-\mathrm{C} \\
\text { asym stretch }\end{array}$ & NA & 571 & 553 & NA & NA \\
\hline & $\begin{array}{l}\text { As-O } \\
\text { stretch }\end{array}$ & 670 & 597 & 578 & 92.2 & 13.8 \\
\hline
\end{tabular}

The purity of $\mathrm{MMA}^{\mathrm{III}}$ was confirmed by comparing its retention time to that of an arsenic standard (Fig. 4.5). The DMA ${ }^{\mathrm{III}}$ characterization was carried out by using the proton NMR (Fig. 4.6).

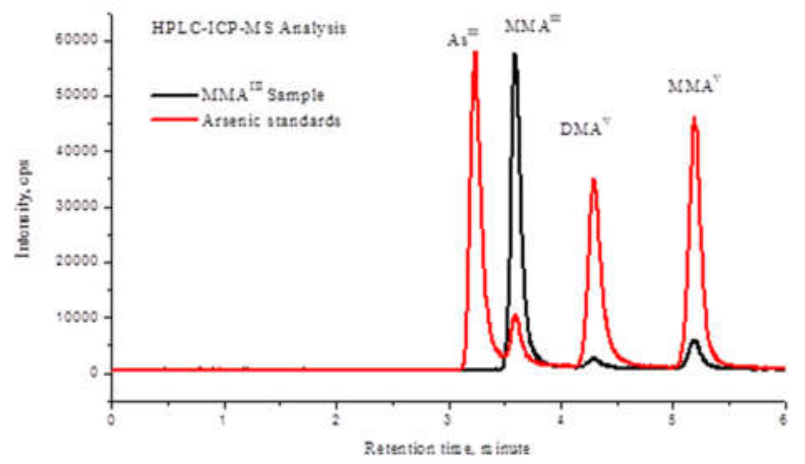

Figure 4.5 HPLC-ICP-MS chromatograms of arsenic standards and MMA synthesized in the laboratory showing the identity and purity

The purity of $\mathrm{MMA}^{\mathrm{III}}$ accoring to the peak area in Fig. 4.5 was about $84 \%$. Small amounts of $\mathrm{MMA}^{\mathrm{V}}$ and $\mathrm{DMA}^{\mathrm{V}}$ were found in the $\mathrm{MMA}^{\mathrm{III}}$ synthesized possibly due to the 
impurity of $\mathrm{MMA}^{\mathrm{V}}$ used as starting material and the incomplete reduction during synthesis.

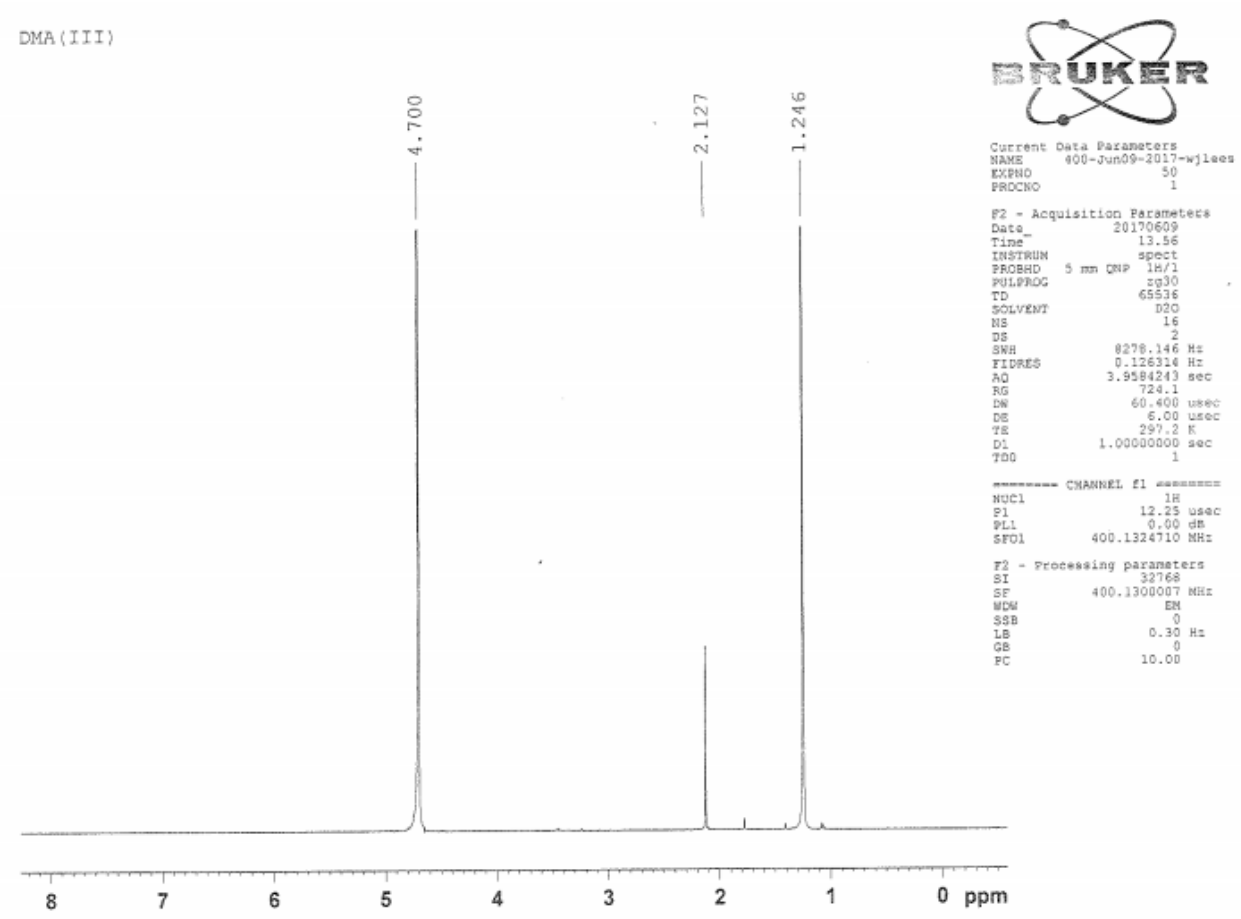

Figure 4.6 Proton NMR spectra for the confirmation of DMAIII synthesized in the laboratory

The chemical shift values in Fig. 4.6 were referenced to the HDO signal at 4.70 ppm. The appearance of DMA ${ }^{\text {III }}$ was confirmed by the chemical shift of the methyl protons at $1.25 \mathrm{ppm}$, which was consistent with the value in the previous report [201].

Both $\mathrm{MMA}^{\mathrm{III}}$ (Fig. 4.4A) and DMA ${ }^{\mathrm{III}}$ (Fig. 4.4B) have characteristic As-C vibration. The DMA ${ }^{\mathrm{III}}$ produced characteristic asymmetric and symmetric vibrational modes of As-C because of the presence of two methyl groups, while MMA ${ }^{\mathrm{III}}$ did not show these bands. Experimental Raman spectra were measured in water using a portable Raman spectrometer. While the Raman signal intensities were weak all vibrational bands were distinguishable from the baseline. In the experimental spectra of MMA ${ }^{\mathrm{III}}$, the 
vibrational band at $599 \mathrm{~cm}^{-1}$ was assigned to the As-C stretch, while the band at $662 \mathrm{~cm}^{-}$ ${ }^{1}$ was assigned to the symmetric vibration of $\mathrm{As}-\mathrm{O}$. The corresponding calculated $\mathrm{As}-\mathrm{C}$ and As-O vibrational frequencies were 572 and $623 \mathrm{~cm}^{-1}$, respectively. No asymmetric vibrational modes of As- $\mathrm{O}$ were found in the experimental spectra, while there was an asymmetric vibration of $\mathrm{As}-\mathrm{O}$ at $596 \mathrm{~cm}^{-1}$ in the calculated Raman spectra. In the experimental Raman spectra of $\mathrm{DMA}^{\mathrm{III}}$, a vibrational band at $613 \mathrm{~cm}^{-1}$ was assigned to the symmetric As-C stretch, and a vibrational band at $670 \mathrm{~cm}^{-1}$ was assigned to the As-O stretch. The corresponding calculated As-C and As-O vibrational frequencies were 565 and $597 \mathrm{~cm}^{-1}$, respectively. The asymmetric As-C vibrational band in DMA ${ }^{\mathrm{III}}$ did not appear as a distinguishable band in the experimental Raman spectra, possiblly a result of the the fact that the symmetric and asymmetric vibrational frequencies were very close as demonstrated from the DFT calculation (565 and $\left.571 \mathrm{~cm}^{-1}\right)$. The disappreance of the assymetric vibration of $\mathrm{As}-\mathrm{O}$ in $\mathrm{DMA}^{\mathrm{III}}$ might casue the error ratio of the As-O frequency in the $\mathrm{DMA}^{\mathrm{III}}$ molecule over $10 \%$. 


\subsubsection{Raman spectra interpretation of DMMTA ${ }^{\mathrm{V}}$ and DMDTA ${ }^{\mathrm{V}}$}

The structure of $\mathrm{DMMTA}^{\mathrm{V}}$ and $\mathrm{DMDTA}^{\mathrm{V}}$ were confirmed by their molecule ion in the mass spectrum (Fig. 4.7).
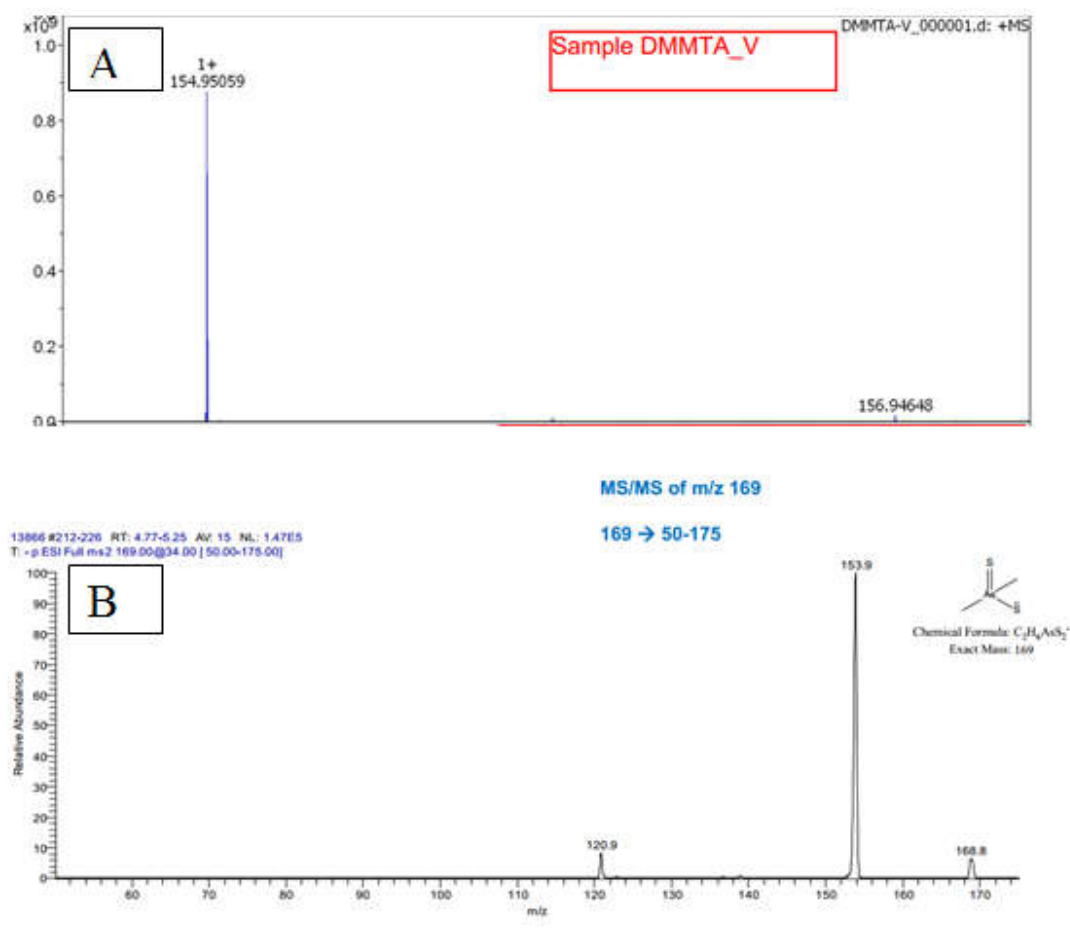

Figure 4.7 Molecular ion peaks confirmation of $\operatorname{DMMTA}^{\mathrm{V}}(\mathrm{A})$ and $\operatorname{DMDTA}^{\mathrm{V}}(\mathrm{B})$ 

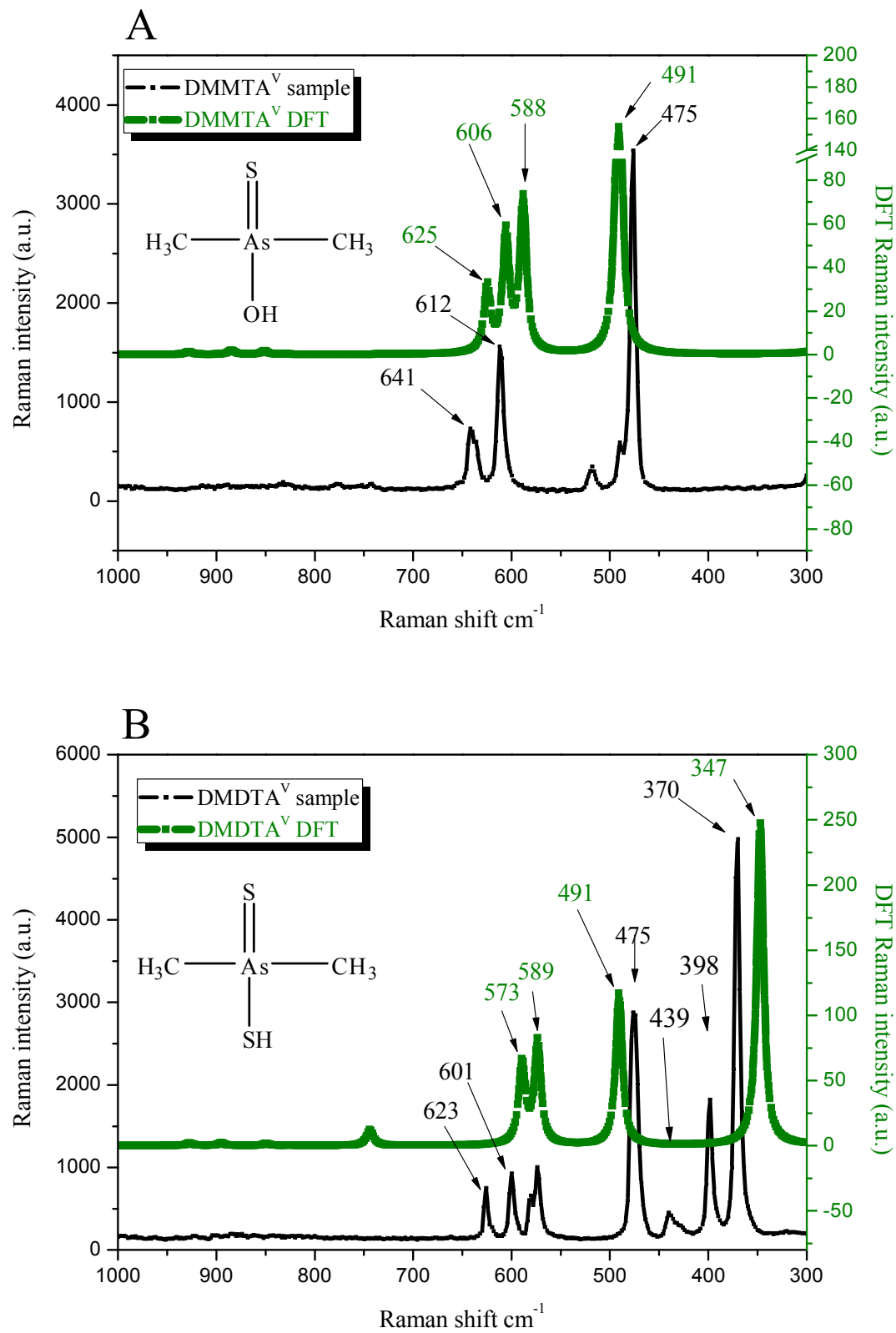

Figure 4.8 Experimental and calculated Raman spectra of $\operatorname{DMMTA}^{\mathrm{V}}(\mathrm{A})$ and $\operatorname{DMDTA}^{\mathrm{V}}(\mathrm{B})$ 
Table 4.5 Vibrational frequencies assignment for $\mathrm{DMMTA}^{\mathrm{V}}$ and DMDTA ${ }^{\mathrm{V}}$

\begin{tabular}{|c|c|c|c|c|c|c|}
\hline Arsenical & $\begin{array}{l}\text { MOLDEN } \\
\text { assignment }\end{array}$ & $\begin{array}{c}\text { Experimental } \\
\text { Raman } \\
\text { frequency } \\
\left(\mathrm{cm}^{-1}\right) \\
\end{array}$ & $\begin{array}{l}\text { Calculated } \\
\text { Raman } \\
\text { frequency } \\
\left(\mathrm{cm}^{-1}\right) \\
\end{array}$ & $\begin{array}{l}\text { Corrected } \\
\text { Raman } \\
\text { frequency } \\
\left(\mathrm{cm}^{-1}\right)\end{array}$ & $\begin{array}{l}\text { Frequency } \\
\text { difference } \\
\left(\mathrm{cm}^{-1}\right)\end{array}$ & $\begin{array}{c}\text { Error } \\
\text { ratio } \\
(\%)\end{array}$ \\
\hline \multirow{4}{*}{ DMMTA $^{\mathrm{V}}$} & $\begin{array}{l}\mathrm{As}=\mathrm{S} \\
\text { stretch }\end{array}$ & 475 & 491 & 475 & 0.2 & 0.1 \\
\hline & $\begin{array}{c}\mathrm{C}-\mathrm{As}-\mathrm{C} \\
\text { sym stretch }\end{array}$ & 612 & 588 & 569 & 43 & 7.0 \\
\hline & $\begin{array}{c}\mathrm{C}-\mathrm{As}-\mathrm{C} \\
\text { asym } \\
\text { stretch }\end{array}$ & NA & 606 & 587 & NA & NA \\
\hline & $\begin{array}{l}\text { As-O } \\
\text { stretch }\end{array}$ & 641 & 625 & 605 & 36 & 5.6 \\
\hline \multirow{4}{*}{ DMDTA $^{\mathrm{V}}$} & $\begin{array}{l}\text { As-S } \\
\text { stretch }\end{array}$ & 370 & 347 & 336 & 34 & 9.2 \\
\hline & $\begin{array}{l}\mathrm{As}=\mathrm{S} \\
\text { stretch }\end{array}$ & 475 & 491 & 475 & 0 & 0.1 \\
\hline & $\begin{array}{c}\mathrm{C}-\mathrm{As}-\mathrm{C} \\
\text { sym stretch }\end{array}$ & 601 & 573 & 555 & 46 & 7.7 \\
\hline & $\begin{array}{c}\mathrm{C}-\mathrm{As}-\mathrm{C} \\
\text { asym } \\
\text { stretch }\end{array}$ & 623 & 589 & 570 & 53 & 8.5 \\
\hline
\end{tabular}

Both DMMTA ${ }^{\mathrm{V}}$ and DMDTA ${ }^{\mathrm{V}}$ have two methyl groups and one $\mathrm{As}=\mathrm{S}$ bond

(Fig. 4.8 and Table 4.5). The major differences between these two compounds are the As-S bond in DMDTA ${ }^{\mathrm{V}}$ and As-O bond in DMMTA ${ }^{\mathrm{V}}$. Both of these compounds had very strong Raman signal in the $1000 \sim 300 \mathrm{~cm}^{-1}$ range. In the experimental spectral results for DMMTA ${ }^{\mathrm{V}}$, three major bands were observed at 475, 612 and $641 \mathrm{~cm}^{-1}$. These were assigned as the $\mathrm{As}-\mathrm{S}$ stretch, the $\mathrm{As}-\mathrm{C}$ symmetric vibration and the $\mathrm{As}-\mathrm{O}$ stretch, respectively (Fig. 4.8A). The corresponding calculated vibrational frequencies of the $\mathrm{As}=\mathrm{S}$ stretch, the As-C symmetric and asymmetric vibrations and the As-O stretches are at 491, 588, 606 and $625 \mathrm{~cm}^{-1}$ respectively. The asymmetric vibrational mode of As $-\mathrm{C}$ did not appear in the experimental spectra, but was observed in the DFT spectra due to the vibration overlap aforementioned. For DMDTA $^{\mathrm{V}}$ (Fig. 4.8B), four major bands were found at $370,475,601$ and $623 \mathrm{~cm}^{-1}$, and they were assigned as the As-S 
stretch, the $\mathrm{As}=\mathrm{S}$ stretch, the $\mathrm{As}-\mathrm{C}$ symmetric vibration and the $\mathrm{As}-\mathrm{C}$ asymmetric vibrations, while the band around 400 and $575 \mathrm{~cm}^{-1}$ may be due to the presence of sodium-DMDTA [266]. There were also some unknown vibrational bands in the DMDTA $^{\mathrm{V}}$ experimental Raman spectra located between $600 \sim 550 \mathrm{~cm}^{-1}$. No effort was made to investigate the appearance of these vibrational bands, and it was assumed that they originated from impurities. The corresponding calculated $\mathrm{As}-\mathrm{S}$ and $\mathrm{As}=\mathrm{S}$ stretches and As-C symmetric and asymmetric vibrational bands are at 347, 491, 573 and 589 $\mathrm{cm}^{-1}$, respectively. The major differences in the Raman spectra between DMMTA ${ }^{\mathrm{V}}$ and DMDTA $^{\mathrm{V}}$ were that the As-S stretch which occurred at $370 \mathrm{~cm}^{-1}$ appeared only in the DMDTA $^{\mathrm{V}}$, while both molecules showed an $\mathrm{As}=\mathrm{S}$ stretch at $475 \mathrm{~cm}^{-1}$. All error ratios between experimental and corrected Raman frequencies were with 10\%.

\subsubsection{Raman spectra interpretation of $\mathrm{DMA}^{\mathrm{III}} \mathrm{GS}$ and $\mathrm{DMA}^{\mathrm{III}} \mathrm{Cys}$}

$\mathrm{DMA}^{\mathrm{III}} \mathrm{Cys}$ was prepared in the laboratory using excess of $\mathrm{DMA}^{\mathrm{V}}$ against Lcysteine and was confirmed by mass spectrometry (Fig. 4.9). As in the diagram, $m / z=226$ was found to be the molecular ion peak, also the mass fragmentation showed ions of losing amine and losing cysteine group from the molecule at $\mathrm{m} / \mathrm{z}=209$ and 137 . 


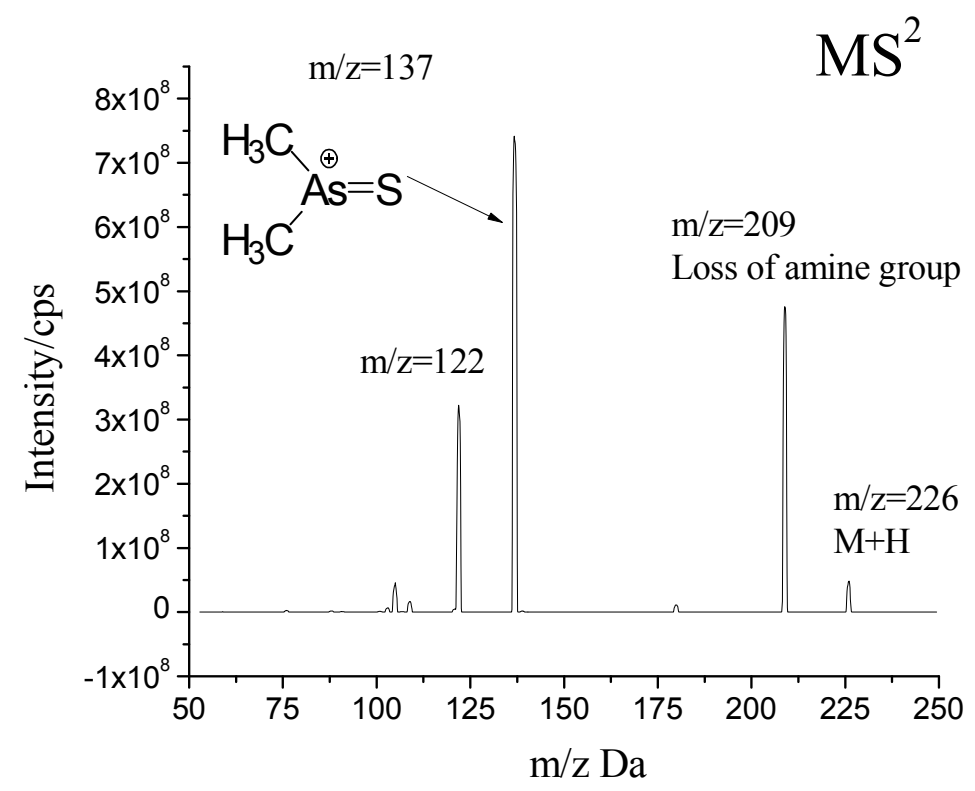

Figure 4.9 Mass spectrum fragmentations of DMA ${ }^{\mathrm{III}}$ Cys by ESI-MS. Mass spectrum fragmentations of DMA ${ }^{\mathrm{III}}$ Cys by ESI-MS. The appearance of $\mathrm{m} / \mathrm{z}$ peak of 226 can be used to confirm the existing of DMA ${ }^{\mathrm{III}} \mathrm{Cys}$ molecule in the reaction product, the fragmentation of $\mathrm{m} / \mathrm{z}=209$ and 137 were the proof of losing the amine group and cysteine group respectively. 


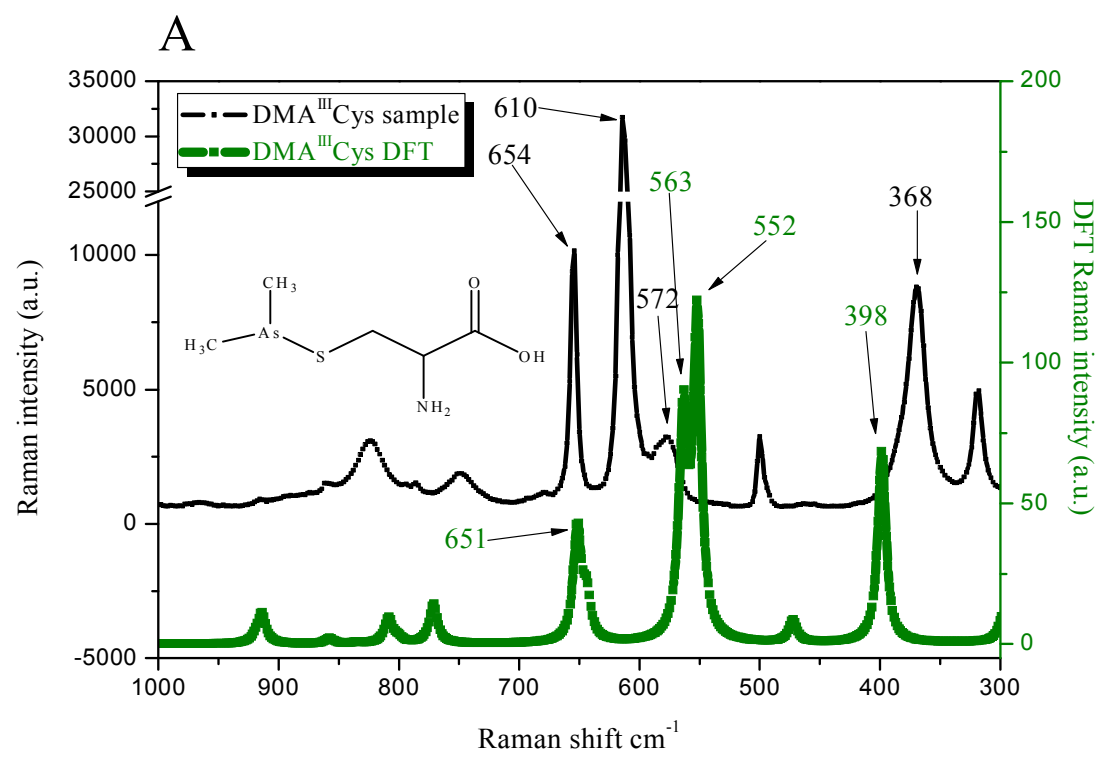

B

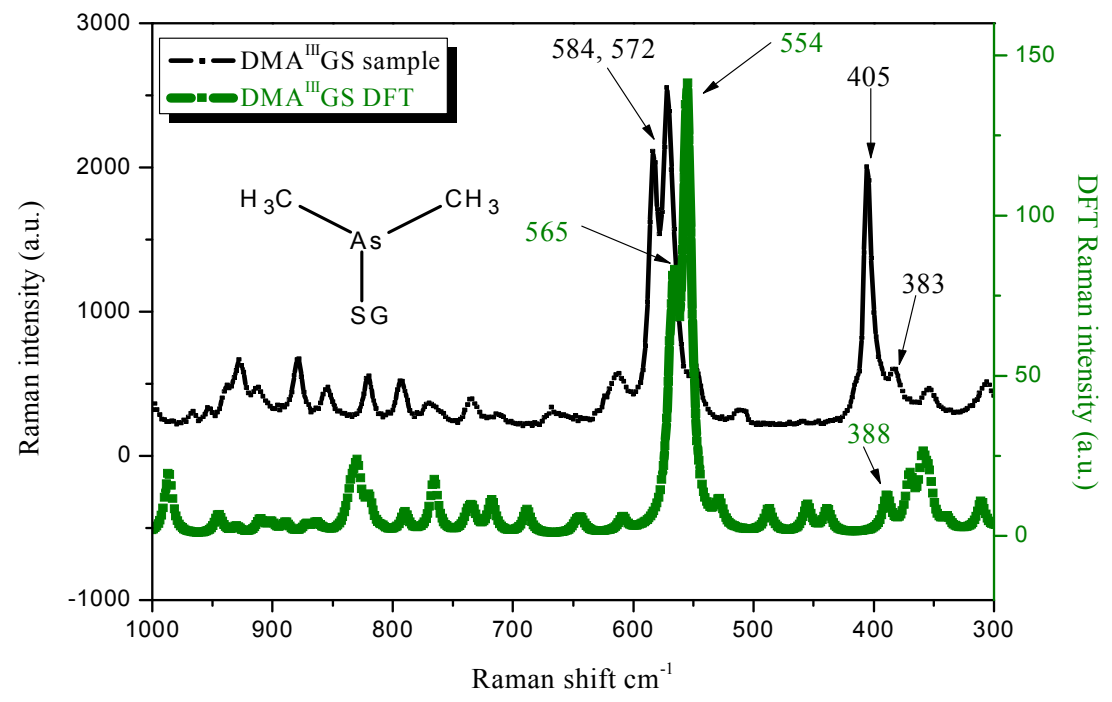

Figure 4.10 Experimental and calculated Raman spectra of $\mathrm{DMA}^{\mathrm{III}} \mathrm{GS}$ (A) and DMA $^{\text {III }}$ Cys (B)

The results for $\mathrm{DMA}^{\mathrm{III}} \mathrm{GS}$ and $\mathrm{DMA}^{\mathrm{III}}$ Cys are summarized in Table 4.6 and Fig. 4.10. $\mathrm{DMA}^{\mathrm{III}} \mathrm{GS}$ was commercially available and used as received. In the experimental spectra of DMA ${ }^{\mathrm{III}} \mathrm{GS}$, three bands were found at 383,572 and $584 \mathrm{~cm}^{-1}$ representing the 
As-S stretch, the As-C symmetric vibration and the As- $\mathrm{C}$ asymmetric vibration, respectively. The corresponding calculated As-S stretch, As-C symmetric and asymmetric stretch appeared at $388,554,565 \mathrm{~cm}^{-1}$. A band at $405 \mathrm{~cm}^{-1}$ also appeared which should be the $\delta(\mathrm{C}-\mathrm{S})$ deformations of the glutathione molecule [267]. Four major bands were observed at $368,572,610$ and $654 \mathrm{~cm}^{-1}$ in the experimental spectra of DMA $^{\mathrm{III}} \mathrm{Cys}$. These were assigned as the As-S stretch, the As-C asymmetric vibration and the As-C symmetric vibration and $\mathrm{C}-\mathrm{S}$ stretch, respectively. The corresponding calculated As-S stretch, As-C symmetric and asymmetric stretch, and $\mathrm{C}-\mathrm{S}$ stretch were $398,552,563$ and $651 \mathrm{~cm}^{-1}$ respectively. Extra bands appeared around 500 and $300 \mathrm{~cm}^{-1}$ in $\mathrm{DMA}^{\mathrm{III}}$ Cys Raman spectra and were not assigned as they may be the result of impurities associated with synthetic procedures. It can be seen that different patterns were observed between the spectra of $\mathrm{DMA}^{\mathrm{III}} \mathrm{GS}$ and $\mathrm{DMA}^{\mathrm{III}} \mathrm{Cys}$, although the only difference in their chemical structure was the complexing ligand. The major difference between the two compounds was that the $\mathrm{C}-\mathrm{S}$ stretch in the DMA ${ }^{\mathrm{III}} \mathrm{Cys}$ appeared around $654 \mathrm{~cm}^{-1}$ (Fig. 4.10A), while it was at $405 \mathrm{~cm}^{-1}$ for $\mathrm{DMA}^{\mathrm{III}} \mathrm{GS}$ due to deformations originated from the $\delta(\mathrm{C}-\mathrm{S})$ (Fig. 4.10B). 
Table 4.6 Vibrational frequencies assignment for DMA ${ }^{\mathrm{III}} \mathrm{GS}$ and DMA ${ }^{\mathrm{III}} \mathrm{Cys}$

\begin{tabular}{|c|c|c|c|c|c|c|}
\hline Arsenical & $\begin{array}{l}\text { MOLDEN } \\
\text { assignment }\end{array}$ & $\begin{array}{l}\text { Experimental } \\
\text { Raman } \\
\text { frequency } \\
\left(\mathrm{cm}^{-1}\right)\end{array}$ & $\begin{array}{l}\text { Calculated } \\
\text { Raman } \\
\text { frequency } \\
\left(\mathrm{cm}^{-1}\right)\end{array}$ & $\begin{array}{l}\text { Corrected } \\
\text { Raman } \\
\text { frequency } \\
\left(\mathrm{cm}^{-1}\right)\end{array}$ & $\begin{array}{l}\text { Frequency } \\
\text { difference } \\
\left(\mathrm{cm}^{-1}\right)\end{array}$ & $\begin{array}{l}\text { Error } \\
\text { ratio } \\
(\%)\end{array}$ \\
\hline \multirow{4}{*}{$\mathrm{DMA}^{\mathrm{III}} \mathrm{Cys}$} & $\begin{array}{l}\text { As-S } \\
\text { stretch }\end{array}$ & 368 & 398 & 385 & 17 & 4.7 \\
\hline & $\begin{array}{l}\mathrm{C}-\mathrm{As}-\mathrm{C} \\
\text { sym } \\
\text { stretch }\end{array}$ & 572 & 552 & 534 & 38 & 6.6 \\
\hline & $\begin{array}{l}\mathrm{C}-\mathrm{As}-\mathrm{C} \\
\text { asym } \\
\text { stretch }\end{array}$ & 610 & 563 & 545 & 65 & 10.7 \\
\hline & $\begin{array}{l}\mathrm{C}-\mathrm{S} \\
\text { stretch }\end{array}$ & 654 & 651 & 630 & 24 & 3.7 \\
\hline \multirow{3}{*}{$\mathrm{DMA}^{\mathrm{III}} \mathrm{GS}$} & $\begin{array}{l}\text { As-S } \\
\text { stretch }\end{array}$ & 383 & 388 & 376 & 7 & 1.9 \\
\hline & $\begin{array}{l}\mathrm{C}-\mathrm{As}-\mathrm{C} \\
\text { sym } \\
\text { stretch }\end{array}$ & 572 & 554 & 536 & 36 & 6.3 \\
\hline & $\begin{array}{l}\mathrm{C}-\mathrm{As}-\mathrm{C} \\
\text { asym } \\
\text { stretch }\end{array}$ & 584 & 565 & 547 & 37 & 6.4 \\
\hline
\end{tabular}

\subsection{Discussion}

4.6.1 Validation of the theoretical computation assisted Raman spectra interpretation method

In this study, a methodology involving the combination of a theoretical simulation of each arsenic compound's fingerprint Raman vibrational modes and its experimental Raman spectra were used for the identification and assignment the spectra of known arsenic compounds. For the validation of our proposed method, the molecule $\mathrm{DMA}^{\mathrm{V}}$ was employed as a standard molecule for the comparison between experimental and calculated Raman frequencies. Computationally, the B3LYP functional with different basis sets was employed for molecular geometry optimization and calculated Raman spectra simulation of all compounds. Among the basis sets used, including 6-31G*, 6- 
$311++\mathrm{G}^{* *}, \mathrm{SDD} / 6-31 \mathrm{G}^{*}, \mathrm{SDD} / 6-311++\mathrm{G}^{* *}$, and def2-TZVPP, the basis set 6$311++\mathrm{G}^{* *}$ yielded the most optimal results in terms of consistency with previous reports regarding molecular geometry optimization and Raman spectral prediction [164, 268]. The average error ratio between experimental and corrected Raman frequencies was about $5.8 \%$, which was lower than the threshold value of $10 \%$, indicating that this method is reliable [260-262]. Discrepancies were found in the Raman frequencies of As- $-\mathrm{O}$ bonds (including single bond and double bonds.) This was most likely due to a systematic error during calculation in which the calculated Raman spectra only considered the isolated molecules in vacuum, while the experimental Raman spectra was obtained in crystalline form with constrained geometry. The RMS value was also an important parameter used to evaluate the performance of a basis set, and the application of a scaling factor was able to significantly reduce the RMS, indicating that the scaling factor was useful in minimizing the discrepancy between experimental and calculated Raman frequencies. The RMS (with scaling factor) for $6-311++\mathrm{G}^{* *}$ was $49 \mathrm{~cm}^{-1}$, being significantly higher than $26 \mathrm{~cm}^{-1}$ in the previous report [260], and this could be due to the limited number of Raman frequencies used for the calculation. Overall, calculated Raman spectra matched the experimental Raman spectra of $\mathrm{DMA}^{\mathrm{V}}$ well with an average error ratio of $5.8 \%$ in Raman frequencies. This result indicated that using the calculated Raman spectra is a reliable method to simulate the experimental Raman spectra and thus this methodology can be used in the identification and assignment of unknown arsenic Raman spectra. 


\subsubsection{Comparison of the basis sets employed for vibrational spectra prediction}

The B3LYP functional with double-zeta or triple-zeta basis sets have achieved admirable accuracy and efficient calculations in other studies [260]. In this work, the B3LYP function with different basis sets was investigated in order to determine how different modes performed when the basis set was changed. Due to the existence of arsenic atom, mixed basis sets of $\mathrm{SDD} / 6-31 \mathrm{G}^{*}$ and $\mathrm{SDD} / 6-311++\mathrm{G}^{* *}$ were also employed to study whether considering relativistic effects could improve accuracy of calculation. The all electron triple-zeta basis set def2-TZVPP was used to in an effort to improve the accuracy of calculations by increasing the size of the basis set. Due to its available crystalline Raman spectra and known structure, $\mathrm{DMA}^{\mathrm{V}}$ was employed as a reference to determine the most precise and efficient basis set.

The accuracy of calculated Raman frequencies depends on the size and quality of the chosen basis set [165]. Different basis sets were employed to process computational geometry optimization of arsenic molecules and simulation of DFT Raman spectra, in which different dispersion, polarization and relativistic effects were considered, in an effort to improve the accuracy of the computational calculation. Two parameters were employed to evaluate the discrepancy of calculated and experimental Raman frequencies, including RMS and numbers of spectra with error ratios over 10\% [260-262].

As shown in Table 4.1A and Table 4.2, the calculated Raman spectra of DMA ${ }^{\mathrm{V}}$ are fundamentally consistent with the experimental data. When no scaling factors were employed in the RMS calculation, the RMS was reduced from 112.4, 90.9 to 60.1 with the basis sets increasing (Table 4.2). The all electron basis set def2-TZVPP also yielded satisfactory outcome with only one error ratio above $10 \%$ and a low RMS value. It was 
found that increasing the dispersion and polarization in basis sets could improve the outcome of RMS calculation. When a scaling factor was applied, the Raman prediction accuracy in all basis sets was improved as shown in Table 4.2, mainly because the calculated Raman frequencies overestimated the experimental data [260]. Earlier work has demonstrated that the error ratio of calculated Raman frequencies against the experimental Raman frequencies is greatly reduced when a scaling factor is applied [269]. The mixed basis sets which take the relativistic effects into consideration such as SDD/6$31 \mathrm{G}^{*}$ and $\mathrm{SDD} / 6-311++\mathrm{G}^{* *}$ did not yield improved results. This is perhaps due to the fact that arsenic belongs in the group of fourth period elements, for which the relativistic effects are not very important in determining vibrational frequencies. Computational efficiency is also a critical parameter in the comparison of performance among these basis sets, although many resource-consuming basis sets are still affordable for most researchers. Extra dispersion and polarization will significantly increase the need for computational resources especially for larger systems such as DMA ${ }^{\mathrm{III}} \mathrm{Cys}$ and DMA ${ }^{\mathrm{III}} \mathrm{GS}$. The time consumption associated with each basis set is shown in Table 4.2. The all electron basis set def2-TZVPP basis set was the most computationally demanding basis set. In addition, the computational costs were greatly reduced when the relativistic effects of the arsenic atom were included during theoretical calculations, since the time consumed in mixed basis sets was shorter than their corresponding counterparts.

Taking the above parameters into consideration, including number of error ratios over $10 \%$, computational efficiency and Raman intensity prediction, $6-311++\mathrm{G}^{* *}$ was the optimal basis set used in this study. It is worth mentioning that the all electron basis 
set def2-TZVPP would be the method of choice if there was no restriction in computational resources.

4.6.3 Fingerprint Raman frequencies of the arsenicals in different chemical environments

The As- $\mathrm{O}$ bond widely exists in inorganic and organic arsenicals present in biological systems and is closely related to arsenic metabolism. As-O vibrational frequencies in pentavalent arsenicals were generally larger than $700 \mathrm{~cm}^{-1}$ except in DMMTA $^{\mathrm{V}}$, whose As-O frequency was $641 \mathrm{~cm}^{-1}$ (Table 4.7). On the other hand, frequencies in trivalent arsenicals were all less than $700 \mathrm{~cm}^{-1}$. The difference in vibrational frequencies for trivalent and pentavalent arsenicals may be partially due to the force constant difference in these two types of compounds. Arsenicals are completely protonated in pentavalent arsenicals since in the solid acidic form, the arsenic atoms are actually attached to hydroxyl groups (As-OH) [149].

Table 4.7 Summary of As-O Raman frequencies

\begin{tabular}{|c|c|c|c|c|c|c|}
\hline Arsenical & Assignment & $\begin{array}{l}\text { Experimental } \\
\text { Raman } \\
\text { spectra } \\
\left(\mathrm{cm}^{-1}\right) \\
\end{array}$ & $\begin{array}{l}\text { Calculated } \\
\text { Raman } \\
\text { frequency } \\
\left(\mathrm{cm}^{-1}\right)\end{array}$ & $\begin{array}{l}\text { Corrected } \\
\text { Raman } \\
\text { frequency } \\
\left(\mathrm{cm}^{-1}\right)\end{array}$ & $\begin{array}{l}\text { Frequency } \\
\text { difference } \\
\left(\mathrm{cm}^{-1}\right)\end{array}$ & $\begin{array}{l}\text { Error } \\
\text { ratio } \\
(\%)\end{array}$ \\
\hline $\mathrm{MMA}^{\mathrm{III}}$ & $\begin{array}{l}\mathrm{O}-\mathrm{As}-\mathrm{O} \\
\text { sym stretch }\end{array}$ & 662 & 661 & 640 & 22 & 3.4 \\
\hline $\mathrm{DMA}^{\mathrm{III}}$ & $\begin{array}{l}\text { As-O } \\
\text { stretch }\end{array}$ & 670 & 597 & 578 & 92 & 13.8 \\
\hline DMMTA $^{\mathrm{V}}$ & $\begin{array}{l}\text { As-O } \\
\text { stretch }\end{array}$ & 641 & 624 & 604 & 37 & 5.8 \\
\hline $\mathrm{iAs}^{\mathrm{V}}$ & $\begin{array}{l}\mathrm{As}-\mathrm{O} \\
\text { stretch }\end{array}$ & 769 [186] & NA & NA & NA & NA \\
\hline $\mathrm{MMA}^{\mathrm{V}}$ & $\begin{array}{l}\mathrm{As}-\mathrm{O}(\mathrm{Va}, \\
\mathrm{Vs})\end{array}$ & $\begin{array}{c}769,780 \\
(\mathrm{Va}, \mathrm{Vs}) \\
{[188]}\end{array}$ & NA & NA & NA & NA \\
\hline $\mathrm{DMA}^{\mathrm{V}}$ & $\begin{array}{l}\text { As-O } \\
\text { stretch }\end{array}$ & 748 & 667 & 646 & 102 & 13.7 \\
\hline
\end{tabular}


However, trivalent arsenicals, such as $\mathrm{MMA}^{\mathrm{III}}$ and $\mathrm{DMA}^{\mathrm{III}}$, are hydrolyzed in excess base and they were fully deprotonated when their Raman spectra were measured. Thus their arsenic atoms were only linked to oxygen atoms (As-O) [149]. The force constant increased from $\mathrm{As}-\mathrm{O}$ to $\mathrm{As}-\mathrm{OH}$ as reported previously [189] and the vibrational frequency was positively correlated to the force constant between the two atoms, i.e. arsenic and oxygen. The valence of arsenicals is likely the major reason for the obvious difference in As-O vibrational frequencies between trivalent and pentavalent arsenic species. A similar result was found in the $\mathrm{Se}-\mathrm{O}$ vibrational frequencies for selenic acid $\left(\mathrm{H}_{2} \mathrm{SeO}_{4}\right)$ and selenous acid $\left(\mathrm{H}_{2} \mathrm{SeO}_{3}\right)$ [270, 271]. Among all pentavalent arsenicals studied, DMMTA ${ }^{\mathrm{V}}$ was the only compound containing an $\mathrm{As}-\mathrm{O}$ bond, and its As-O vibrational frequency was less than $700 \mathrm{~cm}^{-1}$. The only difference between DMA and DMMTA ${ }^{\mathrm{V}}$ was the substitution of the oxygen atom by a sulfur atom. The possible reason for As-O frequency less than $700 \mathrm{~cm}^{-1}$ in the pentavalent DMMTA ${ }^{\mathrm{V}}$ could be the difference in electronegativity between oxygen and sulfur atoms, in which sulfur atom was weaker than oxygen. When sulfur is substituted for oxygen, the electropositivity of the arsenic atom in the molecule DMMTA ${ }^{\mathrm{V}}$ is reduced and thus the force constant of As- $\mathrm{O}$ should also be reduced when compared to DMA ${ }^{\mathrm{V}}$. Furthermore, the calculated Raman frequencies of As-O were mostly smaller than the experimental values. This observation was unusual since the scaling factors were all reported less than 1.0 and the calculated Raman frequencies were generally bigger than the experimental Raman frequencies. However, according to the previous studies, the differences between experimental and corrected frequencies produced a normal distribution [272] and this this result could be interpreted as systematic error during DFT calculation. 
All As-C Raman frequencies were summarized in Table 4.8. There was also a positive correlation between the arsenic valency and the As-C experimental Raman frequencies with the exception of $\mathrm{DMA}^{\mathrm{III}}$, in which the As-C Raman frequencies in pentavalent arsenicals were larger than trivalent ones. Among all Raman spectra, MMA and DMA ${ }^{\mathrm{III}}$ were the only two obtained from aqueous solution (hydrolyzed by $0.1 \mathrm{M}$ $\mathrm{NaOH}$ ) while the rest of the compounds were measured as solid samples. The Raman frequencies of $\mathrm{As}-\mathrm{C}$ in $\mathrm{MMA}^{\mathrm{III}}$ and $\mathrm{DMA}^{\mathrm{III}}$ were significantly higher than those trivalent arsenicals containing sulfur. The reason could be due to the abundant hydroxyl ions existing in the aqueous solution. Only the symmetric vibrational frequencies of As-C were selected for comparison because the symmetric As- $\mathrm{C}$ intensities were found to be stronger than those from asymmetric vibrations in this study. This positive correlation was consistent with the finding by Zingaro et al [273], whose conclusion was based on the summary of Cullen's work [274]. In Cullen's work the carbon-arsenic stretching frequencies in trimethylarsine were from $572 \sim 582 \mathrm{~cm}^{-1}$, while in tetramethylarsonium compounds the frequencies were approximately $650 \mathrm{~cm}^{-1}$ [274]. 
Table 4.8 Summary of As-C Raman frequencies

\begin{tabular}{|c|c|c|c|c|c|c|}
\hline Arsenical & $\begin{array}{l}\text { MOLDEN } \\
\text { assignment }\end{array}$ & $\begin{array}{l}\text { Experimental } \\
\text { Raman } \\
\text { spectra }\left(\mathrm{cm}^{-1}\right)\end{array}$ & $\begin{array}{l}\text { Calculated } \\
\text { Raman } \\
\text { frequency } \\
\left(\mathrm{cm}^{-1}\right)\end{array}$ & $\begin{array}{l}\text { Corrected } \\
\text { Raman } \\
\text { frequency } \\
\left(\mathrm{cm}^{-1}\right)\end{array}$ & $\begin{array}{l}\text { Frequency } \\
\text { difference } \\
\left(\mathrm{cm}^{-1}\right)\end{array}$ & $\begin{array}{l}\text { Error } \\
\text { ratio } \\
(\%)\end{array}$ \\
\hline MMA $^{\text {III }}$ & $\mathrm{As}-\mathrm{C}$ & 599 & 572 & 554 & 45 & 7.6 \\
\hline \multirow{2}{*}{$\mathrm{DMA}^{\mathrm{III}}$} & $\begin{array}{l}\mathrm{C}-\mathrm{As}-\mathrm{C} \\
\text { sym } \\
\text { stretch }\end{array}$ & 613 & 565 & 547 & 66 & 10.8 \\
\hline & $\begin{array}{l}\text { C-As-C } \\
\text { asym } \\
\text { stretch }\end{array}$ & NA & 571 & 553 & NA & NA \\
\hline \multirow{2}{*}{$\mathrm{DMA}^{\mathrm{III}} \mathrm{Cys}$} & $\begin{array}{l}\text { C-As-C } \\
\text { sym } \\
\text { stretch }\end{array}$ & 572 & 552 & 534 & 38 & 6.6 \\
\hline & $\begin{array}{l}\mathrm{C}-\mathrm{As}-\mathrm{C} \\
\text { asym } \\
\text { stretch }\end{array}$ & 610 & 563 & 545 & 65 & 10.7 \\
\hline \multirow{2}{*}{$\mathrm{DMA}^{\mathrm{III}} \mathrm{GS}$} & $\begin{array}{l}\mathrm{C}-\mathrm{As}-\mathrm{C} \\
\text { sym } \\
\text { stretch }\end{array}$ & 572 & 554 & 536 & 36 & 6.3 \\
\hline & $\begin{array}{l}\mathrm{C}-\mathrm{As}-\mathrm{C} \\
\text { asym } \\
\text { stretch }\end{array}$ & 584 & 565 & 547 & 37 & 6.4 \\
\hline \multirow{2}{*}{ DMMTA $^{\mathrm{V}}$} & $\begin{array}{l}\mathrm{C}-\mathrm{As}-\mathrm{C} \\
\text { sym } \\
\text { stretch }\end{array}$ & 612 & 588 & 569 & 43 & 7.0 \\
\hline & $\begin{array}{l}\mathrm{C}-\mathrm{As}-\mathrm{C} \\
\text { asym } \\
\text { stretch }\end{array}$ & NA & 606 & 587 & NA & NA \\
\hline \multirow{2}{*}{ DMDTA $^{\mathrm{V}}$} & $\begin{array}{l}\mathrm{C}-\mathrm{As}-\mathrm{C} \\
\text { sym } \\
\text { stretch }\end{array}$ & 601 & 573 & 555 & 46 & 7.7 \\
\hline & $\begin{array}{l}\text { C-As-C } \\
\text { asym } \\
\text { stretch }\end{array}$ & 623 & 589 & 570 & 53 & 8.5 \\
\hline \multirow{2}{*}{$\mathrm{DMA}^{\mathrm{V}}$} & $\begin{array}{l}\mathrm{C}-\mathrm{As}-\mathrm{C} \\
\text { sym } \\
\text { stretch }\end{array}$ & 611 & 573 & 555 & 56 & 9.2 \\
\hline & $\begin{array}{l}\mathrm{C}-\mathrm{As}-\mathrm{C} \\
\text { asym } \\
\text { stretch }\end{array}$ & 636 & 611 & 591 & 45 & 7.0 \\
\hline
\end{tabular}

Vibrational spectra of several methylated arsenicals containing sulfur have been reported [252, 253, 264-266]. Unfortunately, fingerprint Raman frequencies were not clearly assigned. In a previous study [59], two strong vibrational frequencies located at 
602 and $624 \mathrm{~cm}^{-1}$ were observed in the Raman spectra of $\mathrm{NaS}_{2} \mathrm{AsMe}_{2}$, a thiolated arsenical similar to DMDTA ${ }^{\mathrm{V}}$ used in this study. These Raman frequencies were similar to the As-C bands at 601 and $623 \mathrm{~cm}^{-1}$ observed in DMDTA ${ }^{\mathrm{V}}$, whose Raman spectrum was measured and vibrational frequencies were assigned in this study. Similarly, a vibrational frequency of $612 \mathrm{~cm}^{-1}$ with medium intensity was reported in the Raman spectrum of $\left(\mathrm{CH}_{3}\right)_{2} \mathrm{As}(\mathrm{S}) \mathrm{OAs}(\mathrm{S})\left(\mathrm{CH}_{3}\right)_{2}$ [252]. This is identical to Raman frequency assigned to an As-C symmetrical stretch found in the DMMTA ${ }^{\mathrm{V}}$ shown in this work. It appears that thiolation of these arsenicals did not significantly impact the As-C Raman frequencies, while clear differences in the Raman frequencies of the $\mathrm{As}-\mathrm{O}$ stretch were observed in DMMTA ${ }^{\mathrm{V}}$. Furthermore, no difference in Raman frequency was found in the As-C symmetric frequencies of $\operatorname{DMA}^{\mathrm{V}}\left(611 \mathrm{~cm}^{-1}\right)$ and $\operatorname{DMMTA}^{\mathrm{V}}\left(612 \mathrm{~cm}^{-1}\right)$, which may indicate that thiolation has a limited impact on the $\mathrm{As}-\mathrm{C}$ symmetric frequencies among these aresnicals. However, the As-C symmetric frequency in DMDTA $^{\mathrm{V}}$ was significantly lower than in $\mathrm{DMA}^{\mathrm{V}}$ or $\mathrm{DMMTA}^{\mathrm{V}}$. Therefore, the single As-S bond also has a substantial impact on the As-C symmetric vibrational frequencies. Overall, both reducing the valency of arsenic and the presence of a single As-S bond should reduce As-C symmetric vibrational frequencies. Furthermore arsenic valence has a similar impact on the Raman frequencies of $\mathrm{As}-\mathrm{O}$ and $\mathrm{As}-\mathrm{C}$, resulting in a positive correlation between the arsenic valence and the Raman frequencies of $\mathrm{As}-\mathrm{O}$ or $\mathrm{As}-\mathrm{C}$. It is believed that the electropositivity of arsenic atoms in these pentavalent and trivalent arsenicals has a significant impact, since outer electrons in pentavalent ones are all paired while there is a lone pair of electrons in these trivalent species. The existence of a lone pair of electrons will reduce the electropositivity of arsenic atoms in these trivalent arsenicals [275]. 
Table 4.9 Summary of As-S Raman frequencies

\begin{tabular}{|l|l|l|c|c|c|c|}
\hline Arsenical & $\begin{array}{l}\text { Assignme } \\
\text { nt }\end{array}$ & $\begin{array}{l}\text { Frequency } \\
\left(\mathrm{cm}^{-1}\right)\end{array}$ & $\begin{array}{l}\text { Calculated } \\
\text { Ramanfreq } \\
\text { uency }\left(\mathrm{cm}^{-}\right.\end{array}$ & $\begin{array}{l}\text { Correcte } \\
\text { d Raman } \\
\text { frequenc } \\
\text { y }\left(\mathrm{cm}^{-1}\right)\end{array}$ & $\begin{array}{l}\text { Frequency } \\
\text { difference } \\
\left(\mathrm{cm}^{-1}\right)\end{array}$ & $\begin{array}{l}\text { Error } \\
\text { ratio } \\
(\%)\end{array}$ \\
\hline DMMTA $^{\mathrm{V}}$ & As=S & 475 & 491 & 475 & 0 & 0.1 \\
\hline \multirow{2}{*}{ DMDTA $^{\mathrm{V}}$} & As=S & 475 & 491 & 475 & 0 & 0.1 \\
\cline { 2 - 7 } & As-S & 370 & 347 & 336 & 34 & 9.2 \\
\hline DMA $^{\mathrm{III}} \mathrm{Cys}$ & As-S & 368 & 398 & 385 & 17 & 4.7 \\
\hline DMA $^{\text {III } \mathrm{GS}}$ & As-S & 383 & 388 & 376 & 7 & 1.9 \\
\hline
\end{tabular}

Sulfur-containing arsenicals studied in this study can be categorized into two groups, one is arsenic complexes with sulfur-containing ligands and another is the pentavalent oxoarsenicals with oxygen being substituted by sulfur. Thiol-containing molecules, such as glutathione (GSH), cysteine (Cys) or sulfhydryl containing proteins, are widely present in cellular environment. Trivalent arsenicals can bind to these thiolcontaining molecules and form As-S single bonds. Both single bond (As-S) and double bond $(\mathrm{As}=\mathrm{S})$ can be found in the arsenicals studied (Table 4.9). The Raman frequencies of $\mathrm{As}=\mathrm{S}$ obtained via experiments and DFT calculation were always higher than those found in As-S and this result was consistent with the results reported elsewhere [264, 273, 276]. This observation is the same as the Raman frequency of $\mathrm{As}=\mathrm{O}$ is higher than the As-O in $\mathrm{DMA}^{\mathrm{V}}$. The reason for this pattern is that the force constant between diatoms increases with bond order, [275]. Thus the Raman frequencies of double bonds are always bigger than the respectively single bonds. Similar effects are seen in dithiocacodylate complexes (sulfur containing arsenic chelating ligands $\left.\left(\mathrm{CH}_{3}\right)_{2} \mathrm{AsS}_{2}{ }^{-}\right)$known for their malodor [264, 276-278]. In earlier work, Zingaro had calculated the harmonically vibrational frequencies for $\mathrm{As}-\mathrm{S}$ and $\mathrm{As}=\mathrm{S}$, as 372 and 555 
$\mathrm{cm}^{-1}$ respectively [279]. Raman spectra of dithiocacodylate complexes were usually obtained using their salt accordingly. Due to the distribution of $\pi$-electrons over the whole S-As-S fragment, there were other As-S Raman bands within $400 \sim 500 \mathrm{~cm}^{-1}$ besides the existence of two regular As-S vibrational frequencies [276]. These extra As-S bands were found in DMDTA ${ }^{\mathrm{V}}$ (Fig. 4.8B), between 475 and $370 \mathrm{~cm}^{-1}$. This is important because the DMDTA ${ }^{\mathrm{V}}$ should be in ionic form in the cellular environment.

\subsection{Conclusion}

In this study, we identified and assigned the fingerprint Raman frequencies for some potentially important compounds involved in arsenic metabolism by comparing experimental data with DFT calculations. Of all basis sets employed for DFT Raman spectra simulation, the basis set $6-311++\mathrm{G}^{* *}$ provided the most efficient and accurate result for the standard molecule- $-\mathrm{DMA}^{\mathrm{V}}$ calculation and therefore was utilized for the calculation of all arsenicals. The results of theoretical calculations matched well with the experimental data after an empirical scaling factor applied. The As-O and As-C vibrational frequencies were higher in pentavalent than in trivalent arsenicals. For the thiolated arsenicals, the fingerprint Raman frequencies of As-S (including single and double bonds) were identified, of which the Raman frequency of $\mathrm{As}=\mathrm{S}$ was about 475 $\mathrm{cm}^{-1}$ and the frequency of As-S was around $370 \mathrm{~cm}^{-1}$. This study provides much needed fundamental information on Raman spectrometry for some potentially important arsenicals associated with biological systems. The fingerprint Raman frequencies identified and assigned in these compounds should assist in the development of SERS for arsenic speciation in biological and environmental matrices. 


\section{Chapter 5}

Summary, Significance, and Future Research Directions 


\subsection{Summary}

In this project, arsenic speciation analysis by SERS for $\mathrm{As}^{\mathrm{III}}, \mathrm{As}^{\mathrm{V}}, \mathrm{MMA}^{\mathrm{V}}$ and $\mathrm{DMA}^{\mathrm{V}}$, was carried out under the influence of a cellular matrix. Two different types of silver nanoparticles with different surface coating molecules were employed with varying SERS signal response resulting from different electrostatic interactions with the nanoparticle surface. Each of the four compounds measured generated fingerprint SERS signals, which were distinguishable even in mixed samples. The interactions between arsenic compounds and silver nanoparticles in the colloidal suspensions were studied using adsorption experiments to better understand their respective spectral differences. Increase arsenic adsorption was found in the positively charged nanoparticles, and only the positively charged silver nanoparticles were able to achieve simultaneous detection of arsenicals in the colloidal suspension without separation. However, $\mathrm{MMA}^{\mathrm{V}}$ and $\mathrm{DMA}^{\mathrm{V}}$ had identical SERS signal response in the positively charged nanoparticles. The limit of detections (LOD) was calculated based on a measurement of three times of standard deviation of the blank samples and was determined to be 1.0, 2.8, and $9.9 \mathrm{ppb}$ for $\mathrm{As}^{\mathrm{V}}$, $\mathrm{As}^{\mathrm{III}}$, and $\mathrm{MMA}^{\mathrm{V}} / \mathrm{DMA}^{\mathrm{V}}$, respectively. The work demonstrated that positively charged silver nanoparticles could be a promising SERS substrate for studying arsenic metabolism in a biological matrix, reducing the bias caused by traditional techniques that involve sample preparation and pretreatment.

Secondly, arsenic speciation on silver nanofilms was explored by examining the spectral response of the same four common arsenicals, $\mathrm{As}^{\mathrm{III}}, \mathrm{As}^{\mathrm{V}}, \mathrm{MMA}^{\mathrm{V}}$ and $\mathrm{DMA}^{\mathrm{V}} . \mathrm{A}$ coffee ring effect was observed during the evaporation of a liquid drop on the silver nanofilm. The unique effect coffee ring drove all solute in the droplet towards the edge of 
the coffee ring. At first this coffee ring effect was employed for separation of arsenicals in solutions of different pHs. However, the capillary flow generated in the coffee ring effect was strong and hard to control. No separation was found in these initial experiments and the arsenicals were observed to stack at the edge of coffee ring. To improve separation efficiency, the coffee ring was examined under the influence of surfactants. The introduction of small amounts of surfactants $(0.05 \%)$ caused a continual migration of liquid into the peripheral region of the droplet and increased the migration distance for arsenic separation. This unique coffee ring phenomenon left two concentricring stain patterns after the complete evaporation and it was described as a halo coffee ring effect. The arsenic separation using the halo coffee ring effect was successfully demonstrated in three solutions, including, $0.1 \mathrm{M}$ acetic acid ( $\mathrm{pH}=2.9), 0.1 \mathrm{M}$ phosphate $(\mathrm{pH}=7.5)$ and $0.1 \mathrm{M}$ ammonium formate $(\mathrm{pH}=6.9)$. As the sampling spot moved from the center to the edge of the coffee ring, a clear SERS signal pattern changes were observed at the halo ring region and the inner ring region, indicating that arsenicals were separated along the radius. Moreover, the results illustrated that the electrostatic interactions between arsenicals and the AgNF surface played a major role in the arsenicals separation. This work provides a novel approach for using the coffee ring effect in chemical separations.

Thirdly, fingerprint Raman frequencies of some potentially important arsenic metabolites were assigned and identified by comparing experimental Raman spectra with theoretical Raman spectra obtained from DFT calculations. Most of the arsenicals studied in this work were unstable in ambient conditions, including, $\mathrm{MMA}^{\mathrm{III}}$, DMA ${ }^{\mathrm{III}}, \mathrm{DMMTA}^{\mathrm{V}}$, DMDTA $^{\mathrm{V}}$, DMA ${ }^{\mathrm{III}} \mathrm{GS}$ and DMA ${ }^{\mathrm{III}} \mathrm{GS}$. Through this study, the DFT was demonstrated to 
be helpful in providing simulated Raman spectra and vibrational modes for the identification of spectra from unknown arsenicals. $\mathrm{DMA}^{\mathrm{V}}$ was employed as a representative compound to optimize the computational method. Different basis sets were used to simulate DFT Raman spectra and Raman frequencies were extracted from the calculated results to determine the best basis set. The basis set $6-311++\mathrm{G}^{* *}$ was found to provide the most efficient and precise results for the representative molecule calculation and also used for the computation of all arsenicals. To reduce the discrepancy between theoretical and experimental results, an empirical scaling factor was used and improved matching results were observed. In addition to the identification and assignment of unknown arsenicals' fingerprint Raman spectra, it was also possible to observe Raman frequencies changing rules under the chemical and structural effect. Raman frequencies changing rules were observed. Generally, the $\mathrm{As}-\mathrm{O}$ and $\mathrm{As}-\mathrm{C}$ vibrational frequencies were higher in pentavalent than that in trivalent arsenicals. In addition, the Raman frequencies of $\mathrm{As}=\mathrm{S}$ and $\mathrm{As}-\mathrm{S}$ were measured to be about 475 and $370 \mathrm{~cm}^{-1}$, respectively. The study contributes Raman spectral information for some potentially important arsenicals involved in arsenic metabolism in biological systems. The fingerprint Raman frequencies identified and assigned for these arsenicals could help the method development when using SERS for arsenic speciation in biological and environmental matrices.

\subsection{Significance of this study}

The research reported in this work is original, since no report about SERS studies of organoarsenicals found in cells is available. Arsenic speciation is important in the evaluation of arsenic bioavailability and toxicity towards human health. Currently, most 
analytical methods for the arsenic speciation involve chromatographic separation coupled with mass spectrometry detection. However, the requirement of sample preparation/pretreatment before instrumental measurements could compromise the integrity of arsenicals present in the sample. SERS overcomes many drawbacks of the prevalent analytical methods, because SERS can minimize sample preparation procedures. Previous SERS studies of arsenic were mostly focused on the determination of inorganic arsenic compounds in environmental samples, and organoarsenicals were barely reported. In addition, the study of arsenic speciation in biological matrices using SERS has not previously been explored. This study explored the possibility of arsenic speciation using metallic colloidal suspensions and fundamental parameters were studied. This work included the examination of the interactions between arsenicals and charged nanoparticles, as well as determination arsenicals SERS signals in nanoparticles with different coatings, surface charge, and size in a cellular matrix. In addition, this study unveiled that in addition to aggregation, electrostatic interactions are also important as the nanoparticles aggregation for strong SERS signal generation in the colloidal solutions.

In the second part of this work, a novel arsenic speciation technique using the coffee ring effect was developed. This part of work was highly challenging, because the coffee ring size was very small. It was produced by dropping only $2 \mu \mathrm{L}$ solution onto the AgNF and allowing the strong capillary flow to drive the analytes in the droplet towards the edge. This study demonstrated that two important requirements should be fulfilled for coffee ring separation of small molecules. Firstly, the liquid in the droplet should be able to migrate into peripheral region to increase the arsenicals' migration distance. Secondly, the interactions between the surface and arsenicals should be strong enough to retain the 
arsenicals. To fulfill these two requirements, surfactants were introduced into the droplet to reduce surface tension and permit the liquid to migrate into peripheral region by capillary action. Coating the surface with nanoparticles enhanced the interaction between arsenicals and the surface. The electrostatic interaction played a significant role here since these arsenicals have different deprotonation abilities. This work provides a novel approach for the arsenic speciation by SERS, especially for a small volume of biological samples.

The third part of this work provided valuable information for Raman spectra of potential arsenic metabolites. This work contributes Raman spectra for six potential arsenic metabolites, including $\mathrm{MMA}^{\mathrm{III}}, \mathrm{DMA}^{\mathrm{III}}, \mathrm{DMMTA}^{\mathrm{V}}, \mathrm{DMDTA}^{\mathrm{V}}, \mathrm{DMA}^{\mathrm{III}} \mathrm{GS}$ and DMA $^{\text {III }}$ Cys though the assignment of unknown arsenicals' Raman spectra. Using DMA ${ }^{\mathrm{V}}$ as a representative molecule, detailed Raman spectra analysis and Raman spectra comparisons between experimental and theoretical results were performed. The theoretical calculations were found to provide useful Raman spectra predictions for the experimental spectra, and computational simulations also provided detailed molecular movements for the identification of vibrational modes. Since most of relevant arsenicals were not commercially available, these compounds were mostly synthesized in the laboratory. A new method was validated to synthesize $\mathrm{DMA}^{\mathrm{III}} \mathrm{Cys}$ using excessive of $\mathrm{DMA}^{\mathrm{V}}$ against L-cysteine. This approach avoided serious signal interference of the byproduct cystine, which is usualy produced in the typical synthetic recipe using a molar excess of L-cysteine against DMA ${ }^{\mathrm{V}}$. 


\subsection{Future research directions}

The experiments involving arsenic speciation with silver colloidal suspensions developed in this work was a preliminary study. Only four commonly existing redoxinert arsenicals were selected to demonstrate the possibility of arsenic speciation using SERS. The study should be expanded to investigate other potential arsenic metabolites found in arsenic metabolism, such as $\mathrm{MMA}^{\mathrm{III}}, \mathrm{DMA}^{\mathrm{III}}, \mathrm{DMMTA}^{\mathrm{V}}, \mathrm{DMDTA}^{\mathrm{V}}, \mathrm{DMA}^{\mathrm{III}} \mathrm{GS}$ and DMA ${ }^{\mathrm{III}}$ Cys.. However, the stability of these unstable arsenicals in term of SERS enhancement in different colloidal suspensions should be studied prior to this work.

There are additional potential arsenic metabolites found in arsenic metabolism, such as, monomethyl monothioarsonate $\left(\mathrm{MMMTA}^{\mathrm{V}}\right)$, monomethyldithioarsonic acid $\left(\mathrm{MMDTA}^{\mathrm{V}}\right)$, monomethylarsenic diglutathione $\left(\mathrm{MMA}^{\mathrm{III}}(\mathrm{GS})_{2}\right)$, dimethylarsinothioyl glutathione (DMMTA $\left.{ }^{\mathrm{V}}(\mathrm{GS})\right)$ etc. It would be useful to study their Raman spectra by employing density functional theory computation.

Arsenic speciation inside cells can potentially be carried out by using the introduction of nanoparticles into cells. Gold nanoshells may be fabricated and optimized to permit determination of molecules in vivo. Protection of the surface of these gold nanoshells may be necessary to prevent biomolecules from attaching to their surface when in the cytoplasm.

Arsenic speciation using the coffee ring effect, especially the halo coffee ring effect, could be expanded towards use in other elements' speciation. In addition complementary studies on the mechanism of this halo coffee ring effect should be carried out to obtain comprehensive understanding of the phenomenon. For example, the question about surface tension changes of the sessile droplet during the formation of halo 
coffee ring stain need to be studied. It would be interesting to discover the correlation between the droplet surface tension and the size of the halo region to improve separation. Meanwhile, the interactions between analytes and the surface can be improved by using nanoparticles with different surface coatings or though the applying of AgNF surface modification. 


\section{Reference}

[1] K.A. Francesconi and D. Kuehnelt, Arsenic compounds in the environment, Environmental chemistry of arsenic, in: W.T.W.T. Frankenberger, Marcel Dekker. New York, pp. 51-94.

[2] I. Gaballah, N. Menad, D. Hartmann, G. Lyaudet, and P. Michel, Decontamination as important step towards recycling, Resources, Conservation and Recycling. 10 (1994) 107-116.

[3] J. Nriagu, P. Bhattacharya, A. Mukherjee, J. Bundschuh, R. Zevenhoven, and R. Loeppert, Arsenic in soil and groundwater: an overview, Trace Metals and other Contaminants in the Environment, in, pp. 3-60.

[4] P. Smedley and D. Kinniburgh, A review of the source, behaviour and distribution of arsenic in natural waters, Applied geochemistry. 17 (2002) 517-568.

[5] R. Nickson, J. McArthur, W. Burgess, K.M. Ahmed, P. Ravenscroft, and M. Rahman, Arsenic poisoning of Bangladesh groundwater, Nature. 395 (1998) 338.

[6] A. Krysiak and A. Karczewska, Arsenic extractability in soils in the areas of former arsenic mining and smelting, SW Poland, Sci. Total Environ. 379 (2007) 190-200.

[7] A. Davis, M.V. Ruby, and P.D. Bergstrom, Bioavailability of Arsenic and Lead in Soils from the Butte, Montana, Mining District, Environ. Sci. Technol. 26 (1992) 461-468.

[8] J. Lever, Paget's disease of bone in Lancashire and arsenic pesticide in cotton mill wastewater: a speculative hypothesis, Bone. 31 (2002) 434-436.

[9] R. Wauchope, Fixation of arsenical herbicides, phosphate, and arsenate in alluvial soils, J. Environ. Qual. 4 (1975) 355-358.

[10] M. Flues, I.M. Sato, M.A. Scapin, M.E.B. Cotrim, and I.M.C. Camargo, Toxic elements mobility in coal and ashes of Figueira coal power plant, Brazil, Fuel. 103 (2013) 430-436.

[11] B.P. Jackson and P.M. Bertsch, Determination of arsenic speciation in poultry wastes by IC-ICP-MS, Environ. Sci. Technol. 35 (2001) 4868-4873.

[12] H. Garelick, H. Jones, A. Dybowska, and E. Valsami-Jones, Arsenic pollution sources, Reviews of Environmental Contamination and Toxicology, in, Springer. New York, pp. 17-60. 
[13] J. Liu, B. Zheng, H.V. Aposhian, Y. Zhou, M.-L. Chen, A. Zhang, and M.P. Waalkes, Chronic arsenic poisoning from burning high-arsenic-containing coal in Guizhou, China, Environ. Health Persp. 110 (2002) 119-122.

[14] G. Sun, Arsenic contamination and arsenicosis in China, Toxicol. Appl. Pharmacol. 198 (2004) 268-271.

[15] B.K. Mandal and K.T. Suzuki, Arsenic round the world: a review, Talanta. 58 (2002) 201-235.

[16] J.O. Nrtagu, Arsenic poisoning through the ages, Environmental chemistry of arsenic, in: J. William T. Frankenberger, Marcel Dekker. New York, pp. 1-26.

[17] S. Strom, F.D.A. Bans Three Arsenic Drugs Used in Poultry and Pig Feeds, The New York Times. (2013). http://www.nytimes.com/2013/10/02/business/fdabans-three-arsenic-drugs-used-in-poultry-and-pig-feeds.html

[18] K. Lew, J.P. Acker, S. Gabos, and X.C. Le, Biomonitoring of Arsenic in Urine and Saliva of Children Playing on Playgrounds Constructed from Chromated Copper Arsenate-Treated Wood, Environ. Sci. Technol. 44 (2010) 3986-3991.

[19] J.A. Hingston, C.D. Collins, R.J. Murphy, and J.N. Lester, Leaching of chromated copper arsenate wood preservatives: a review, Environ. Pollut. 111 (2001) 53-66.

[20] A.H. Smith, E.O. Lingas, and M. Rahman, Contamination of drinking-water by arsenic in Bangladesh: a public health emergency, Bull. W.H.O. 78 (2000) 10931103 .

[21] T.-H.L.Y.-L. Huang and M.-Y. Wang, Arsenic species in drinking water, hair, fingernails, and urine of patients with blackfoot disease, J. Toxicol. Env. Heal. A. 53 (1998) 85-93.

[22] K. Farzana Akter, Z. Chen, L. Smith, D. Davey, and R. Naidu, Speciation of arsenic in ground water samples: A comparative study of CE-UV, HG-AAS and LC-ICP-MS, Talanta. 68 (2005) 406-415.

[23] C.-p. Liu, C.-1. Luo, Y. Gao, F.-b. Li, L.-w. Lin, C.-a. Wu, and X.-d. Li, Arsenic contamination and potential health risk implications at an abandoned tungsten mine, southern China, Environ. Pollut. 158 (2010) 820-826.

[24] G. Li, G.-X. Sun, P.N. Williams, L. Nunes, and Y.-G. Zhu, Inorganic arsenic in Chinese food and its cancer risk, Environ. Int. 37 (2011) 1219-1225.

[25] S. Kar, J.P. Maity, J.-S. Jean, C.-C. Liu, C.-W. Liu, J. Bundschuh, and H.-Y. Lu, Health risks for human intake of aquacultural fish: Arsenic bioaccumulation and contamination, Journal of Environmental Science and Health Part A. 46 (2011) 1266-1273. 
[26] A.Q. Shah, T.G. Kazi, J.A. Baig, M.B. Arain, H.I. Afridi, G.A. Kandhro, S.K. Wadhwa, and N.F. Kolachi, Determination of inorganic arsenic species (As3+ and As5+) in muscle tissues of fish species by electrothermal atomic absorption spectrometry (ETAAS), Food Chem. 119 (2010) 840-844.

[27] G. Okkenhaug, Y.-G. Zhu, J. He, X. Li, L. Luo, and J. Mulder, Antimony (Sb) and arsenic (As) in Sb mining impacted paddy soil from Xikuangshan, China: differences in mechanisms controlling soil sequestration and uptake in rice, Environ. Sci. Technol. 46 (2012) 3155-3162.

[28] M.A. Rahman and H. Hasegawa, High levels of inorganic arsenic in rice in areas where arsenic-contaminated water is used for irrigation and cooking, Sci. Total Environ. 409 (2011) 4645-4655.

[29] Z. Cheng, K.-C. Chen, K.-B. Li, X.-P. Nie, S.C. Wu, C.K.-C. Wong, and M.-H. Wong, Arsenic contamination in the freshwater fish ponds of Pearl River Delta: bioaccumulation and health risk assessment, Environ. Sci. Pollut. R. (2012) 1-12.

[30] Tsuruta, Hamada, Mochida, Nakagawa, Kobayashi, and Ishii, Merkel cell carcinoma, Bowen's disease and chronic occupational arsenic poisoning, Br. J. Dermatol. 139 (1998) 291-294.

[31] A.M. Lee and J.F. Fraumeni, Arsenic and respiratory cancer in man: an occupational study, J. Natl. Cancer Inst. 42 (1969) 1045-1052.

[32] J.G. Farmer and L.R. Johnson, Assessment of Occupational Exposure to Inorganic Arsenic Based on Urinary Concentrations and Speciation of Arsenic, Br. J. Ind. Med. 47 (1990) 342-348.

[33] Y. Yoshimura, Y. Endo, Y. Shimoda, K. Yamanaka, and G. Endo, Acute Arsine Poisoning Confirmed by Speciation Analysis of Arsenic Compounds in the Plasma and Urine by HPLC-ICP-MS, J. Occup. Health. 53 (2011) 45-49.

[34] L. Järup, G. Pershagen, and S. Wall, Cumulative arsenic exposure and lung cancer in smelter workers: a dose-response study, Am. J. Ind. Med. 15 (1989) 31-41.

[35] V.C. Pandey, J.S. Singh, R.P. Singh, N. Singh, and M. Yunus, Arsenic hazards in coal fly ash and its fate in Indian scenario, Resources, Conservation and Recycling. 55 (2011) 819-835.

[36] D. Jolliffe, A history of the use of arsenicals in man, J. R. Soc. Med. 86 (1993) 287-289.

[37] T.D. Zhang, [Treatment of acute granulocytic leukemia with "Ai ling No. 1"-clinical analysis and experimental research], Zhong xi yi jie he za zhi $=$ Chinese journal of modern developments in traditional medicine. 4 (1984) 19-20. 
[38] S.A. Lerman, T.W. Clarkson, and R.J. Gerson, Arsenic uptake and metabolism by liver cells is dependent on arsenic oxidation state, Chem-Biol Interact. 45 (1983) 401-406.

[39] M. Vahter, Species differences in the metabolism of arsenic compounds, Appl. Organomet. Chem. 8 (1994) 175-182.

[40] R.-N. Huang and T.-C. Lee, Cellular uptake of trivalent arsenite and pentavalent arsenate in KB cells cultured in phosphate-free medium, Toxicol. Appl. Pharmacol. 136 (1996) 243-249.

[41] C.-H. Tseng, C.-K. Chong, C.-J. Chen, and T.-Y. Tai, Dose-response relationship between peripheral vascular disease and ingested inorganic arsenic among residents in blackfoot disease endemic villages in Taiwan, Atherosclerosis. 120 (1996) 125-133.

[42] R.N. Ratnaike, Acute and chronic arsenic toxicity, Postgrad. Med. J. 79 (2003) 391-396.

[43] M.R. Karagas, T.D. Tosteson, J. Blum, B. Klaue, J.E. Weiss, V. Stannard, V. Spate, and J.S. Morris, Measurement of low levels of arsenic exposure: a comparison of water and toenail concentrations, Am. J. Epidemiol. 152 (2000) 84-90.

[44] B.K. Mandal, Y. Ogra, and K.T. Suzuki, Speciation of arsenic in human nail and hair from arsenic-affected area by HPLC-inductively coupled argon plasma mass spectrometry, Toxicol. Appl. Pharmacol. 189 (2003) 73-83.

[45] A.G. Gault, H.A. Rowland, J.M. Charnock, R.A. Wogelius, I. Gomez-Morilla, S. Vong, M. Leng, S. Samreth, M.L. Sampson, and D.A. Polya, Arsenic in hair and nails of individuals exposed to arsenic-rich groundwaters in Kandal province, Cambodia, Sci. Total Environ. 393 (2008) 168-176.

[46] M.M. Rahman, J.C. Ng, and R. Naidu, Chronic exposure of arsenic via drinking water and its adverse health impacts on humans, Environ. Geochem. Health. 31 Suppl 1 (2009) 189-200.

[47] J.C. States, S. Srivastava, Y. Chen, and A. Barchowsky, Arsenic and Cardiovascular Disease, Toxicol. Sci. 107 (2009) 312-323.

[48] Y. Chen, J.H. Graziano, F. Parvez, M. Liu, V. Slavkovich, T. Kalra, M. Argos, T. Islam, A. Ahmed, M. Rakibuz-Zaman, R. Hasan, G. Sarwar, D. Levy, A. van Geen, and H. Ahsan, Arsenic exposure from drinking water and mortality from cardiovascular disease in Bangladesh: prospective cohort study, BMJ. 342 (2011). 
[49] M. Rahman, M. Tondel, S.A. Ahmad, and O. Axelson, Diabetes Mellitus Associated with Arsenic Exposure in Bangladesh, Am. J. Epidemiol. 148 (1998) 198-203.

[50] A. Navas-Acien, E.K. Silbergeld, R. Pastor-Barriuso, and E. Guallar, ARsenic exposure and prevalence of type 2 diabetes in us adults, JAMA. 300 (2008) 814822.

[51] J.K. Levin-Scherz, J.D. Patrick, F.H. Weber, and C. Garabedian, Acute arsenic ingestion, Ann. Emerg. Med. 16 (1987) 702-704.

[52] A. Dueñas-Laita, M. Pérez-Miranda, M.A. González-López, J.C. Martín-Escudero, M. Ruiz-Mambrilla, and J. Blanco-Varela, Acute arsenic poisoning, The Lancet. 365 (2005) 1982.

[53] D. Trachootham, W. Lu, M.A. Ogasawara, R.D. Nilsa, and P. Huang, Redox regulation of cell survival, Antioxid. Redox Signal. 10 (2008) 1343-1374.

[54] H.P. Indo, M. Davidson, H.-C. Yen, S. Suenaga, K. Tomita, T. Nishii, M. Higuchi, Y. Koga, T. Ozawa, and H.J. Majima, Evidence of ROS generation by mitochondria in cells with impaired electron transport chain and mitochondrial DNA damage, Mitochondrion. 7 (2007) 106-118.

[55] T. Finkel and N.J. Holbrook, Oxidants, oxidative stress and the biology of ageing, Nature. 408 (2000) 239-247.

[56] Y. Kobayashi, X. Cui, and S. Hirano, Stability of arsenic metabolites, arsenic triglutathione $[\mathrm{As}(\mathrm{GS})(3)]$ and methylarsenic diglutathione [CH3As(GS)(2)], in rat bile, Toxicology. 211 (2005) 115-123.

[57] C. Jung and A. Rothstein, Arsenate uptake and release in relation to the inhibition of transport and glycolysis in yeast, Biochem. Pharmacol. 14 (1965) 1093-1112.

[58] J. Pi, H. Yamauchi, Y. Kumagai, G. Sun, T. Yoshida, H. Aikawa, C. HopenhaynRich, and N. Shimojo, Evidence for induction of oxidative stress caused by chronic exposure of Chinese residents to arsenic contained in drinking water, Environ. Health Persp. 110 (2002) 331-336.

[59] S. Das, A. Santra, S. Lahiri, and D.G. Mazumder, Implications of oxidative stress and hepatic cytokine (TNF- $\alpha$ and IL-6) response in the pathogenesis of hepatic collagenesis in chronic arsenic toxicity, Toxicol. Appl. Pharmacol. 204 (2005) 1826.

[60] G.M. Cooper, The central role of enzymes as biological catalysts, (2000). 
[61] T. Samikkannu, C.-H. Chen, L.-H. Yih, A.S. Wang, S.-Y. Lin, T.-C. Chen, and K.-Y. Jan, Reactive oxygen species are involved in arsenic trioxide inhibition of pyruvate dehydrogenase activity, Chem. Res. Toxicol. 16 (2003) 409-414.

[62] I. Lehnman, DNA ligase: structure, mechanism, and function, Science. 186 (1974) 790-797.

[63] C. Bernstein, A.R. Prasad, V. Nfonsam, and H. Bernstein, DNA damage, DNA repair and cancer, New Research Directions in DNA Repair. InTech. doi: $10.5772 / 53919$

[64] M. Kessel, S.X. Liu, A. Xu, R. Santella, and T.K. Hei, Arsenic induces oxidative DNA damage in mammalian cells, Mol. Cell. Biochem. 234 (2002) 301-308.

[65] T. Schwerdtle, I. Walter, I. Mackiw, and A. Hartwig, Induction of oxidative DNA damage by arsenite and its trivalent and pentavalent methylated metabolites in cultured human cells and isolated DNA, Carcinogenesis. 24 (2003) 967-974.

[66] K.T. Kitchin and S. Ahmad, Oxidative stress as a possible mode of action for arsenic carcinogenesis, Toxicol. Lett. 137 (2003) 3-13.

[67] A. Hartwig, Carcinogenicity of metal compounds: possible role of DNA repair inhibition, Toxicol. Lett. 102 (1998) 235-239.

[68] A.D. Kligerman, C.L. Doerr, A.H. Tennant, K. Harrington-Brock, J.W. Allen, E. Winkfield, P. Poorman-Allen, B. Kundu, K. Funasaka, and B.C. Roop, Methylated trivalent arsenicals as candidate ultimate genotoxic forms of arsenic: induction of chromosomal mutations but not gene mutations, Environ. Mol. Mutagen. 42 (2003) 192-205.

[69] S. Nesnow, B.C. Roop, G. Lambert, M. Kadiiska, R.P. Mason, W.R. Cullen, and M.J. Mass, DNA damage induced by methylated trivalent arsenicals is mediated by reactive oxygen species, Chem. Res. Toxicol. 15 (2002) 1627-1634.

[70] M.J. Mass, A. Tennant, B.C. Roop, W.R. Cullen, M. Styblo, D.J. Thomas, and A.D. Kligerman, Methylated trivalent arsenic species are genotoxic, Chem. Res. Toxicol. 14 (2001) 355-361.

[71] Y. Yoshimura, Y. Endo, Y. Shimoda, K. Yamanaka, and G. Endo, Acute Arsine Poisoning Confirmed by Speciation Analysis of Arsenic Compounds in the Plasma and Urine by HPLC-ICP-MS, J. Occup. Health. 53 (2010) 45-49.

[72] Y.-J. Hsieh and S.-J. Jiang, Application of HPLC-ICP-MS and HPLC-ESI-MS Procedures for Arsenic Speciation in Seaweeds, J. Agric. Food Chem. 60 (2012) 2083-2089. 
[73] P. Alava, F. Tack, G.D. Laing, and T.V. de Wiele, HPLC-ICP-MS method development to monitor arsenic speciation changes by human gut microbiota, Biomed. Chromatogr. 26 (2012) 524-533.

[74] J.L. Gómez-Ariza, D. Sánchez-Rodas, I. Giráldez, and E. Morales, A comparison between ICP-MS and AFS detection for arsenic speciation in environmental samples, Talanta. 51 (2000) 257-68.

[75] C. Niegel, S.A. Pfeiffer, M. Grundmann, U. Arroyo-Abad, J. Mattusch, and F.-M. Matysik, Fast separations by capillary electrophoresis hyphenated to electrospray ionization time-of-flight mass spectrometry as a tool for arsenic speciation analysis, Analyst. 137 (2012) 1956-1962.

[76] L. Liu, B. He, Z. Yun, J. Sun, and G. Jiang, Speciation analysis of arsenic compounds by capillary electrophoresis on-line coupled with inductively coupled plasma mass spectrometry using a novel interface, J. Chromatogr. A. 1304 (2013) 227-233.

[77] G. Yang, J. Xu, J. Zheng, X. Xu, W. Wang, L. Xu, G. Chen, and F. Fu, Speciation analysis of arsenic in Mya arenaria Linnaeus and Shrimp with capillary electrophoresis-inductively coupled plasma mass spectrometry, Talanta. 78 (2009) 471-6.

[78] A.A. Ammann, Arsenic speciation by gradient anion exchange narrow bore ion chromatography and high resolution inductively coupled plasma mass spectrometry detection, J. Chromatogr. A. 1217 (2010) 2111-2116.

[79] V. Dufailly, M. Nicolas, J.R. Payot, and E. Poitevin, Validation of a method for arsenic speciation in food by ion chromatography-inductively coupled plasma/mass spectrometry after ultrasonic-assisted enzymatic extraction, J. AOAC Int. 94 (2011) 947-958.

[80] J. Morton and E. Leese, Arsenic speciation in clinical samples: urine analysis using fast micro-liquid chromatography ICP-MS, Anal. Bioanal. Chem. 399 (2011) 1781-1788.

[81] U. Arroyo-Abad, S. Lischka, C. Piechotta, J. Mattusch, and T. Reemtsma, Determination and identification of hydrophilic and hydrophobic arsenic species in methanol extract of fresh cod liver by RP-HPLC with simultaneous ICP-MS and ESI-Q-TOF-MS detection, Food Chem. 141 (2013) 3093-3102.

[82] S.D. Conklin, M.W. Fricke, P.A. Creed, and J.T. Creed, Investigation of the $\mathrm{pH}$ effects on the formation of methylated thio-arsenicals, and the effects of $\mathrm{pH}$ and temperature on their stability, J. Anal. Atom. Spectrom. 23 (2008) 711-716. 
[83] H.R. Hansen, A. Raab, M. Jaspars, B.F. Milne, and J. Feldmann, SulfurContaining Arsenical Mistaken for Dimethylarsinous Acid [DMA(III)] and Identified as a Natural Metabolite in Urine: Major Implications for Studies on Arsenic Metabolism and Toxicity, Chem. Res. Toxicol. 17 (2004) 1086-1091.

[84] T. Hayakawa, Y. Kobayashi, X. Cui, and S. Hirano, A new metabolic pathway of arsenite: arsenic-glutathione complexes are substrates for human arsenic methyltransferase Cyt19, Arch. Toxicol. 79 (2005) 183-191.

[85] K. Rehman and H. Naranmandura, Arsenic metabolism and thioarsenicals, Metallomics : integrated biometal science. 4 (2012) 881-892.

[86] M.G. Mason, A.S. Ball, B.J. Reeder, G. Silkstone, P. Nicholls, and M.T. Wilson, Extracellular heme peroxidases in actinomycetes: a case of mistaken identity, Appl. Environ. Microbiol. 67 (2001) 4512-4519.

[87] R. Kar and C.B. Sharma, Bilirubin peak can be mistaken as Hb Bart's or Hb H on High-performance liquid chromatography, Hemoglobin. 35 (2011) 171-174.

[88] P.G. Smith, I. Koch, R.A. Gordon, D.F. Mandoli, B.D. Chapman, and K.J. Reimer, X-ray Absorption Near-Edge Structure Analysis of Arsenic Species for Application to Biological Environmental Samples, Environ. Sci. Technol. 39 (2004) 248-254.

[89] C.S. Kim, G.E. Brown Jr, and J.J. Rytuba, Characterization and speciation of mercury-bearing mine wastes using X-ray absorption spectroscopy, Sci. Total Environ. 261 (2000) 157-168.

[90] I.J. Pickering, G.E. Brown, and T.K. Tokunaga, Quantitative Speciation of Selenium in Soils Using X-Ray-Absorption Spectroscopy, Environ. Sci. Technol. 29 (1995) 2456-2459.

[91] D. Paktunc, A. Foster, and G. Laflamme, Speciation and characterization of arsenic in Ketza River mine tailings using x-ray absorption spectroscopy, Environ. Sci. Technol. 37 (2003) 2067-2074.

[92] P.G. Smith, I. Koch, R.A. Gordon, D.F. Mandoli, B.D. Chapman, and K.J. Reimer, $\mathrm{X}$-ray absorption near-edge structure analysis of arsenic species for application to biological environmental samples, Environ. Sci. Technol. 39 (2005) 248-254.

[93] R. Andrahennadi, M. Wayland, and I.J. Pickering, Speciation of selenium in stream insects using x-ray absorption Spectroscopy, Environ. Sci. Technol. 41 (2007) 7683-7687.

[94] A.L. Foster, G.E. Brown, T.N. Tingle, and G.A. Parks, Quantitative arsenic speciation in mine tailings using X-ray absorption spectroscopy, Am. Mineral. 83 (1998) 553-568. 
[95] A.A. Meharg, E. Lombi, P.N. Williams, K.G. Scheckel, J. Feldmann, A. Raab, Y.G. Zhu, and R. Islam, Speciation and localization of arsenic in white and brown rice grains, Environ. Sci. Technol. 42 (2008) 1051-1057.

[96] E. Lombi, K.G. Scheckel, J. Pallon, A.M. Carey, Y.G. Zhu, and A.A. Meharg, Speciation and distribution of arsenic and localization of nutrients in rice grains, New Phytol. 184 (2009) 193-201.

[97] T. Bacquart, G. Devès, and R. Ortega, Direct speciation analysis of arsenic in subcellular compartments using micro-X-ray absorption spectroscopy, Environ. Res. 110 (2010) 413-416.

[98] T. Bacquart, G. Devès, A. Carmona, R. Tucoulou, S. Bohic, and R. Ortega, Subcellular Speciation Analysis of Trace Element Oxidation States Using Synchrotron Radiation Micro-X-ray Absorption Near-Edge Structure, Anal. Chem. 79 (2007) 7353-7359.

[99] J.P. Scaffidi, M.K. Gregas, V. Seewaldt, and T. Vo-Dinh, SERS-based plasmonic nanobiosensing in single living cells, Anal. Bioanal. Chem. 393 (2009) 1135-1141.

[100] D.I. Ellis and R. Goodacre, Metabolic fingerprinting in disease diagnosis: biomedical applications of infrared and Raman spectroscopy, Analyst. 131 (2006) 875-885.

[101] F. Inscore, C. Shende, A. Sengupta, H. Huang, and S. Farquharson, Detection of drugs of abuse in saliva by surface-enhanced Raman spectroscopy (SERS), Appl. Spectrosc. 65 (2011) 1004-1008.

[102] M. Casella, A. Lucotti, M. Tommasini, M. Bedoni, E. Forvi, F. Gramatica, and G. Zerbi, Raman and SERS recognition of $\beta$-carotene and haemoglobin fingerprints in human whole blood, Spectrochim. Acta A. 79 (2011) 915-919.

[103] D. Drescher, T. Büchner, D. McNaughton, and J. Kneipp, SERS reveals the specific interaction of silver and gold nanoparticles with hemoglobin and red blood cell components, Phys. Chem. Chem. Phys. 15 (2013) 5364-5373.

[104] C. Leordean, V. Canpean, and S. Astilean, Surface-enhanced Raman scattering (SERS) analysis of urea trace in urine, fingerprint, and tear samples, Spectrosc. Lett. 45 (2012) 550-555.

[105] R. Stosch, A. Henrion, D. Schiel, and B. Güttler, Surface-enhanced Raman scattering based approach for quantitative determination of creatinine in human serum, Anal. Chem. 77 (2005) 7386-7392.

[106] B. ZHENG, J.-c. DONG, L.-z. SU, M. Meng, Y.-j. ZHANG, and J.-f. LI, SurfaceEnhanced Raman Spectroscopy Study of Fresh Human Urine: A Preliminary Study, Spectrosc. Spect. Anal. 36 (2016) 1987-1991. 
[107] Y. Wang, J. Chen, Y. Wu, Y. Chen, J. Pan, J. Lei, Y. Chen, L. Sun, S. Feng, and R. Chen. Surface-enhanced Raman spectroscopy of creatinine in silver colloid. SPIE (2012), doi:10.1117/12.918990.

[108] T.L. Wang, K.C. Hui-hua, and H.-h. Lu. SERS quantitative urine creatinine measurement of human subject. SPIE (2005), doi: 10.1117/12.591393.

[109] S. Feng, D. Lin, J. Lin, B. Li, Z. Huang, G. Chen, W. Zhang, L. Wang, J. Pan, and R. Chen, Blood plasma surface-enhanced Raman spectroscopy for non-invasive optical detection of cervical cancer, Analyst. 138 (2013) 3967-3974.

[110] G. Del Mistro, S. Cervo, E. Mansutti, R. Spizzo, A. Colombatti, P. Belmonte, R. Zucconelli, A. Steffan, V. Sergo, and A. Bonifacio, Surface-enhanced Raman spectroscopy of urine for prostate cancer detection: a preliminary study, Anal. Bioanal. Chem. 407 (2015) 3271-3275.

[111] S. Feng, J. Lin, Z. Huang, G. Chen, W. Chen, Y. Wang, R. Chen, and H. Zeng, Esophageal cancer detection based on tissue surface-enhanced Raman spectroscopy and multivariate analysis, Appl. Phys. Lett. 102 (2013), doi:10.1063/1.4789996.

[112] C.J. Saatkamp, M.L. de Almeida, J.A.M. Bispo, A.L.B. Pinheiro, A.B. Fernandes, and L. Silveira, Quantifying creatinine and urea in human urine through Raman spectroscopy aiming at diagnosis of kidney disease, J. Biomed. Opt. 21 (2016) 037001-037001. doi:10.1117/1.JBO.21.3.037001.

[113] J. Wang, Y.Y. Zeng, J.Q. Lin, L. Lin, X.C. Wang, G.N. Chen, Z.F. Huang, B.H. Li, H.S. Zeng, and R. Chen, SERS spectroscopy and multivariate analysis of globulin in human blood, Laser Phys. 24 (2014), doi: 10.1088/1054$660 X / 24 / 6 / 065602$.

[114] Z. Huang, X. Chen, Y. Chen, J. Chen, M. Dou, S. Feng, H. Zeng, and R. Chen, Raman spectroscopic characterization and differentiation of seminal plasma, J. Biomed. Opt. 16 (2011), doi:10.1117/1.3650310.

[115] Z. Ye, H. Meizhen, W. Kehui, S. Biao, W. Yang, C. Jie, L. Xi, L. Xia, L. Lulu, and H. Gaozhong, Urine surface-enhanced Raman spectroscopy for non-invasive diabetic detection based on a portable Raman spectrometer, Laser Phys. Lett. 13 (2016), doi: 10.1088/1612-2011/13/6/065604.

[116] M. Li, Y. Du, F. Zhao, J. Zeng, C. Mohan, and W.-C. Shih, Reagent-and separation-free measurements of urine creatinine concentration using stamping surface enhanced Raman scattering (S-SERS), Biomed. Opt. Express. 6 (2015) 849-858. 
[117] S. Shanmukh, L. Jones, J. Driskell, Y. Zhao, R. Dluhy, and R.A. Tripp, Rapid and sensitive detection of respiratory virus molecular signatures using a silver nanorod array SERS substrate, Nano Lett. 6 (2006) 2630-2636.

[118] H. Wang, N. Malvadkar, S. Koytek, J. Bylander, W.B. Reeves, and M.C. Demirel, Quantitative analysis of creatinine in urine by metalized nanostructured parylene, J. Biomed. Opt. 15 (2010), doi:10.1117/1.3369002.

[119] L. Guerrini, E. Pazos, C. Penas, M.E. Vazquez, J.L. Mascarenas, and R.A. Alvarez-Puebla, Highly sensitive SERS quantification of the oncogenic protein cJun in cellular extracts, J. Am. Chem. Soc. 135 (2013) 10314-10317.

[120] M.Y. Sha, H. Xu, M.J. Natan, and R. Cromer, Surface-enhanced Raman scattering tags for rapid and homogeneous detection of circulating tumor cells in the presence of human whole blood, J. Am. Chem. Soc. 130 (2008) 17214-17215.

[121] H. Chon, S. Lee, S.W. Son, C.H. Oh, and J. Choo, Highly sensitive immunoassay of lung cancer marker carcinoembryonic antigen using surface-enhanced Raman scattering of hollow gold nanospheres, Anal. Chem. 81 (2009) 3029-3034.

[122] B.-H. Jun, M.S. Noh, G. Kim, H. Kang, J.-H. Kim, W.-J. Chung, M.-S. Kim, Y.K. Kim, M.-H. Cho, and D.H. Jeong, Protein separation and identification using magnetic beads encoded with surface-enhanced Raman spectroscopy, Anal. Biochem. 391 (2009) 24-30.

[123] H. Chon, S. Lee, S.-Y. Yoon, S.-I. Chang, D.W. Lim, and J. Choo, Simultaneous immunoassay for the detection of two lung cancer markers using functionalized SERS nanoprobes, Chem. Commun. 47 (2011) 12515-12517.

[124] V.K. Gupta, N. Atar, M.L. Yola, M. Eryılmaz, H. Torul, U. Tamer, İ.H. Boyac1, and Z. Üstündağ, A novel glucose biosensor platform based on Ag@ AuNPs modified graphene oxide nanocomposite and SERS application, J. Colloid. Interf. Sci. 406 (2013) 231-237.

[125] K.V. Kong, Z. Lam, W.K.O. Lau, W.K. Leong, and M. Olivo, A transition metal carbonyl probe for use in a highly specific and sensitive sers-based assay for glucose, J. Am. Chem. Soc. 135 (2013) 18028-18031.

[126] S.J. Greaves and W.P. Griffith, Surface-enhanced Raman scattering (SERS) from silver colloids of vanadate, phosphate and arsenate, J. Raman Spectrosc. 19 (1988) 503-507.

[127] J. Li, L. Chen, T. Lou, and Y. Wang, Highly sensitive SERS detection of As3+ ions in aqueous media using glutathione functionalized silver nanoparticles, ACS Appl. Mater. Inter. 3 (2011) 3936-3941. 
[128] J. Du, J. Cui, and C. Jing, Rapid in situ identification of arsenic species using a portable $\mathrm{Fe}_{3} \mathrm{O}_{4} @$ Ag SERS sensor, Chem. Commun. 50 (2014) 347-349.

[129] L. Ye, G. Wen, J. Dong, Y. Luo, Q. Liu, A. Liang, and Z. Jiang, A simple labelfree rhodamine $6 \mathrm{G}$ SERS probe for quantitative analysis of trace As $3+$ in an aptamer-nanosol, RSC Adv. 4 (2014) 32960-32964.

[130] L. Song, K. Mao, X. Zhou, and J. Hu, A novel biosensor based on Au@ Ag coreshell nanoparticles for SERS detection of arsenic (III), Talanta. 146 (2016) 285290.

[131] W. Wang, Y. Yin, Z. Tan, and J. Liu, Coffee-ring effect-based simultaneous SERS substrate fabrication and analyte enrichment for trace analysis, Nanoscale. 6 (2014) 9588-9593.

[132] M. Mulvihill, A. Tao, K. Benjauthrit, J. Arnold, and P. Yang, Surface-Enhanced Raman Spectroscopy for Trace Arsenic Detection in Contaminated Water, Angew. Chem. Int. Ed. 47 (2008) 6456-6460.

[133] Z.H. Xu, J.M. Hao, F.S. Li, and X.G. Meng, Surface-enhanced Raman spectroscopy of arsenate and arsenite using Ag nanofilm prepared by modified mirror reaction, J. Colloid. Interf. Sci. 347 (2010) 90-95.

[134] D. Gloria, G. Moran, and D.B. Hibbert. Detection of Arsenobetaine: A Step Towards SERS-based Arsenic Speciation. AIP conference proceedings. 510 (2010), doi: 10.1063/1.3482645

[135] J. Olavarría-Fullerton, S. Wells, W. Ortiz-Rivera, M.J. Sepaniak, and M.A. De Jesus, Surface-Enhanced Raman Scattering (SERS) Characterization of Trace Organoarsenic Antimicrobials Using Silver/Polydimethylsiloxane Nanocomposites, Appl. Spectrosc. 65 (2011) 423-428.

[136] J.M. Hao, M.J. Han, Z.H. Xu, J.W. Li, and X.G. Meng, Fabrication and evolution of multilayer silver nanofilms for surface-enhanced Raman scattering sensing of arsenate, Nanoscale Res. Lett. 6 (2011) 263.

[137] Z. Xu, X. Meng, Y. Zhang, and F. Li, Effects and mechanisms of water matrix on surface-enhanced Raman scattering analysis of arsenite on silver nanofilm, Colloid. Surface. A. 497 (2016) 117-125.

[138] Z. Xu, C. Jing, J. Hao, C. Christodoulatos, G.P. Korfiatis, F. Li, and X. Meng, Effect of bonding interactions between arsenate and silver nanofilm on surfaceenhanced Raman scattering sensitivity, J. Phys. Chem. C. 116 (2011) 325-329.

[139] A.M. Fales, H. Yuan, and T. Vo-Dinh, Development of Hybrid Silver-Coated Gold Nanostars for Nonaggregated Surface-Enhanced Raman Scattering, J Phys Chem C 118 (2014) 3708-3715. 
[140] H. Yuan, C.G. Khoury, C.M. Wilson, G.A. Grant, A.J. Bennett, and T. Vo-Dinh, In vivo particle tracking and photothermal ablation using plasmon-resonant gold nanostars, Nanomed. Nanotechnol. Biol. Med. 8 (2012) 1355-1363.

[141] H. Park, S. Lee, L. Chen, E.K. Lee, S.Y. Shin, Y.H. Lee, S.W. Son, C.H. Oh, J.M. Song, S.H. Kang, and J. Choo, SERS imaging of HER2-overexpressed MCF7 cells using antibody-conjugated gold nanorods, Phys. Chem. Chem. Phys. 11 (2009) 7444-7449.

[142] Z. Xu, J. Hao, F. Li, and X. Meng, Surface-enhanced Raman spectroscopy of arsenate and arsenite using $\mathrm{Ag}$ nanofilm prepared by modified mirror reaction, J. Colloid. Interf. Sci. 347 (2010) 90-95.

[143] R. Haresh, D. Philip, and G. Aruldhas, Infrared, Raman, and SERS spectra of betaine arsenate, Spectrosc. Lett. 28 (1995) 11-28.

[144] Z. Ji, X. Jin, S. George, T. Xia, H. Meng, X. Wang, E. Suarez, H. Zhang, E.M. Hoek, H. Godwin, A.E. Nel, and J.I. Zink, Dispersion and stability optimization of TiO2 nanoparticles in cell culture media, Environ. Sci. Technol. 44 (2010) 7309-7314.

[145] C. Schulze, C. Schulze, A. Kroll, C. Schulze, A. Kroll, C.-M. Lehr, U.F. Schäfer, K. Becker, J. Schnekenburger, C. Schulze Isfort, R. Landsiedel, and W. Wohlleben, Not ready to use - overcoming pitfalls when dispersing nanoparticles in physiological media, Nanotoxicology. 2 (2008) 51-61.

[146] M.P. Monopoli, D. Walczyk, A. Campbell, G. Elia, I. Lynch, F.B. Bombelli, and K.A. Dawson, Physical-chemical aspects of protein corona: relevance to in vitro and in vivo biological impacts of nanoparticles, J. Am. Chem. Soc. 133 (2011) 2525-2534.

[147] M.J. Han, J.M. Hao, Z.H. Xu, and X.G. Meng, Surface-enhanced Raman scattering for arsenate detection on multilayer silver nanofilms, Anal. Chim. Acta. 692 (2011) 96-102.

[148] J. Hao, Z. Xu, M.-J. Han, S. Xu, and X. Meng, Surface-enhanced Raman scattering analysis of perchlorate using silver nanofilms deposited on copper foils, Colloid. Surface. A. 366 (2010) 163-169.

[149] K. Müller, V.S.T. Ciminelli, M.S.S. Dantas, and S. Willscher, A comparative study of $\mathrm{As}(\mathrm{III})$ and $\mathrm{As}(\mathrm{V})$ in aqueous solutions and adsorbed on iron oxyhydroxides by Raman spectroscopy, Water Res. 44 (2010) 5660-5672.

[150] R.D. Deegan, O. Bakajin, T.F. Dupont, G. Huber, S.R. Nagel, and T.A. Witten, Capillary flow as the cause of ring stains from dried liquid drops, Nature. 389 (1997) 827-829. 
[151] J.T. Wen, C.-M. Ho, and P.B. Lillehoj, Coffee ring aptasensor for rapid protein detection, Langmuir. 29 (2013) 8440-8446.

[152] E. Kočišová, M. Procházka, and H. Šípová, Thiol-modified gold-coated glass as an efficient hydrophobic substrate for drop coating deposition Raman (DCDR) technique, J. Raman Spectrosc. 47 (2016) 1394-1396.

[153] P. Šimáková, M. Procházka, and E. Kočišová, SERS Microspectroscopy of Biomolecules on Dried Ag Colloidal Drops, Spectrosc. Int. J. 27 (2012) 449-453.

[154] X. Pan, J. Dong, Y. Li, X. Sun, C. Yuan, and W. Qian, The strategy of two-scale interface enrichment for constructing ultrasensitive SERS substrates based on the coffee ring effect of AgNP@ $\beta$-CD, RSC Adv. 6 (2016) 29586-29591.

[155] S. Yang, X. Dai, B.B. Stogin, and T.-S. Wong, Ultrasensitive surface-enhanced Raman scattering detection in common fluids, P. Natl. Acad. Sci. 113 (2016) 268273.

[156] D.M. Kuncicky, B.G. Prevo, and O.D. Velev, Controlled assembly of SERS substrates templated by colloidal crystal films, J. Mater. Chem. 16 (2006) 12071211.

[157] J. Xu, J. Du, C. Jing, Y. Zhang, and J. Cui, Facile detection of polycyclic aromatic hydrocarbons by a surface-enhanced Raman scattering sensor based on the $\mathrm{Au}$ coffee ring effect, ACS Appl. Mater. Inter. 6 (2014) 6891-6897.

[158] P.J. Yunker, T. Still, M.A. Lohr, and A. Yodh, Suppression of the coffee-ring effect by shape-dependent capillary interactions, Nature. 476 (2011) 308-311.

[159] L. Cui, J. Zhang, X. Zhang, L. Huang, Z. Wang, Y. Li, H. Gao, S. Zhu, T. Wang, and B. Yang, Suppression of the coffee ring effect by hydrosoluble polymer additives, ACS Appl. Mater. Inter. 4 (2012) 2775-2780.

[160] T.S. Wong, T.H. Chen, X.Y. Shen, and C.M. Ho, Nanochromatography Driven by the Coffee Ring Effect, Anal. Chem. 83 (2011) 1871-1873.

[161] N.R. Devlin, K. Loehr, and M.T. Harris, The separation of two different sized particles in an evaporating droplet, AICHE J. 61 (2015) 3547-3556.

[162] D. Michalska and R. Wysokiński, The prediction of Raman spectra of platinum (II) anticancer drugs by density functional theory, Chem. Phys. Lett. 403 (2005) 211-217.

[163] D. Michalska, W. Zierkiewicz, D.C. Bienko, W. Wojciechowski, and T. ZeegersHuyskens, "Troublesome" Vibrations of Aromatic Molecules in Second-Order Möller-Plesset and Density Functional Theory Calculations: Infrared Spectra of Phenol and Phenol-OD Revisited, J. Phys. Chem. A. 105 (2001) 8734-8739. 
[164] S.Z. Fairchild, C.F. Bradshaw, W. Su, and S.K. Guharay, Predicting Raman spectra using density functional theory, Appl. Spectrosc. 63 (2009) 733-741.

[165] M.P. Andersson and P. Uvdal, New scale factors for harmonic vibrational frequencies using the B3LYP density functional method with the triple- $\zeta$ basis set 6-311+ G (d, p), J. Phys. Chem. A. 109 (2005) 2937-2941.

[166] R.G. Freeman, K.C. Grabar, K.J. Allison, and R.M. Bright, Self-assembled metal colloid monolayers: an approach to SERS substrates, Science. 267 (1995) 1629.

[167] C. Steinmaus, Y. Yuan, D. Kalman, O.A. Rey, C.F. Skibola, D. Dauphine, A. Basu, K.E. Porter, A. Hubbard, and M.N. Bates, Individual differences in arsenic metabolism and lung cancer in a case-control study in Cordoba, Argentina, Toxicol. Appl. Pharmacol. 247 (2010) 138-145.

[168] L.M. Knobeloch, K.M. Zierold, and H.A. Anderson, Association of arseniccontaminated drinking-water with prevalence of skin cancer in Wisconsin's Fox River Valley, Journal of Health, Population and Nutrition. 24 (2011) 206-213.

[169] C. Ferreccio, A.H. Smith, V. Durán, T. Barlaro, H. Benítez, R. Valdés, J.J. Aguirre, L.E. Moore, J. Acevedo, M.I. Vásquez, L. Pérez, Y. Yuan, J. Liaw, K.P. Cantor, and C. Steinmaus, Case-Control Study of Arsenic in Drinking Water and Kidney Cancer in Uniquely Exposed Northern Chile, Am. J. Epidemiol. 178 (2013) 813-818.

[170] K. Jomova and M. Valko, Advances in metal-induced oxidative stress and human disease, Toxicology. 283 (2011) 65-87.

[171] Y. Sun, G. Liu, and Y. Cai, Thiolated arsenicals in arsenic metabolism: Occurrence, formation, and biological implications, J. Environ. Sci. 49 (2016) 5973.

[172] T.W. Gebel, Arsenic methylation is a process of detoxification through accelerated excretion, Int J Hyg Envir Heal. 205 (2002) 505-508.

[173] H.V. Aposhian, E.S. Gurzau, X.C. Le, A. Gurzau, S.M. Healy, X. Lu, M. Ma, L. Yip, R.A. Zakharyan, R.M. Maiorino, R.C. Dart, M.G. Tircus, D. GonzalezRamirez, D.L. Morgan, D. Avram, and M.M. Aposhian, Occurrence of Monomethylarsonous Acid in Urine of Humans Exposed to Inorganic Arsenic, Chem. Res. Toxicol. 13 (2000) 693-697.

[174] X.C. Le, X. Lu, M. Ma, W.R. Cullen, H.V. Aposhian, and B. Zheng, Speciation of key arsenic metabolic intermediates in human urine, Anal. Chem. 72 (2000) 5172-5177. 
[175] B. Moe, H. Peng, X. Lu, B. Chen, L.W.L. Chen, S. Gabos, X.-F. Li, and X.C. Le, Comparative cytotoxicity of fourteen trivalent and pentavalent arsenic species determined using real-time cell sensing, J. Environ. Sci. 49 (2016) 113-124.

[176] L. Yehiayan, M. Pattabiraman, K. Kavallieratos, X.T. Wang, L.H. Boise, and Y. Cai, Speciation, formation, stability and analytical challenges of human arsenic metabolites, J. Anal. Atom. Spectrom. 24 (2009) 1397-1405.

[177] L.W.L. Chen, X. Lu, and X.C. Le, Complementary chromatography separation combined with hydride generation-inductively coupled plasma mass spectrometry for arsenic speciation in human urine, Anal. Chim. Acta. 675 (2010) 71-75.

[178] Z. Gong, X. Lu, W.R. Cullen, and X. Chris Le, Unstable trivalent arsenic metabolites, monomethylarsonous acid and dimethylarsinous acid, J. Anal. Atom. Spectrom. 16 (2001) 1409-1413.

[179] Z. Sun, J. Du, and C. Jing, Recent progress in detection of mercury using surface enhanced Raman spectroscopy - A review, J. Environ. Sci. 39 (2016) 134-143.

[180] J. Hao, M.-J. Han, S. Han, X. Meng, T.-L. Su, and Q.K. Wang, SERS detection of arsenic in water: A review, J. Environ. Sci. 36 (2015) 152-162.

[181] P. Lee and D. Meisel, Adsorption and surface-enhanced Raman of dyes on silver and gold sols, J. Phys. Chem. 86 (1982) 3391-3395.

[182] D. van Lierop, Z. Krpetic, L. Guerrini, I.A. Larmour, J.A. Dougan, K. Faulds, and D. Graham, Positively charged silver nanoparticles and their effect on surfaceenhanced Raman scattering of dye-labelled oligonucleotides, Chem. Commun. 48 (2012) 8192-8194.

[183] L. Yehiayan, N. Membreno, S. Matulis, L.H. Boise, and Y. Cai, Extraction tool and matrix effects on arsenic speciation analysis in cell lines, Anal. Chim. Acta. 699 (2011) 187-192.

[184] T.M. Loehr and R.A. Plane, Raman spectra and structures of arsenious acid and arsenites in aqueous solution, Inorg. Chem. 7 (1968) 1708-1714.

[185] S.C. Myneni, S.J. Traina, G.A. Waychunas, and T.J. Logan, Experimental and theoretical vibrational spectroscopic evaluation of arsenate coordination in aqueous solutions, solids, and at mineral-water interfaces, Geochim. Cosmochim. Acta. 62 (1998) 3285-3300.

[186] F.K. Vansant, B.J. Van Der Veken, and H.O. Desseyn, Vibrational analysis of arsenic acid and its anions: I. Description of the Raman spectra, J. Mol. Struct. 15 (1973) 425-437. 
[187] F. Vansant, B. Van der Veken, and M. Herman, Vibrational analysis of dimethylarsinic acid, Spectrochim. Acta A-M. 30 (1974) 69-78.

[188] F. Vansant, B. Van der Veken, and M. Herman, The vibrational analysis of methylarsonic acid, trideuteromethylarsonic acid and their anions, J. Mol. Struct. 35 (1976) 191-200.

[189] S. Cowen, M. Duggal, T. Hoang, and H.A. Al-Abadleh, Vibrational spectroscopic characterization of some environmentally important organoarsenicals-A guide for understanding the nature of their surface complexes, Can. J. Chem. 86 (2008) 942-950.

[190] H. Wang, C.S. Levin, and N.J. Halas, Nanosphere arrays with controlled sub-10$\mathrm{nm}$ gaps as surface-enhanced Raman spectroscopy substrates, J. Am. Chem. Soc. 127 (2005) 14992-14993.

[191] C. Jing, X. Meng, S. Liu, S. Baidas, R. Patraju, C. Christodoulatos, and G.P. Korfiatis, Surface complexation of organic arsenic on nanocrystalline titanium oxide, J. Colloid. Interf. Sci. 290 (2005) 14-21.

[192] P. Gao and M.J. Weaver, Surface-enhanced Raman spectroscopy as a probe of adsorbate-surface bonding: benzene and monosubstituted benzenes adsorbed at gold electrodes, J. Phys. Chem. 89 (1985) 5040-5046.

[193] A. Campion and P. Kambhampati, Surface-enhanced Raman scattering, Chem. Soc. Rev. 27 (1998) 241-250.

[194] X.-M. Lin, Y. Cui, Y.-H. Xu, B. Ren, and Z.-Q. Tian, Surface-enhanced Raman spectroscopy: substrate-related issues, Anal. Bioanal. Chem. 394 (2009) 17291745 .

[195] M. Rahman, S. Laurent, N. Tawil, L.H. Yahia, and M. Mahmoudi, Nanoparticle and Protein Corona, Protein-Nanoparticle Interactions: The Bio-Nano Interface, in, Springer Berlin Heidelberg. Berlin, Heidelberg, pp. 21-44.

[196] P. Ball, Fluid dynamics - How coffee leaves its mark, Nature. 389 (1997) 788-788.

[197] R. Fondecave and F.B. Wyart, Wetting laws for polymer solutions, EPL (Europhysics Letters). 37 (1997) 115.

[198] H.Y. Erbil, Evaporation of pure liquid sessile and spherical suspended drops: A review, Adv. Colloid Interfac. 170 (2012) 67-86.

[199] E. Tekin, P.J. Smith, and U.S. Schubert, Inkjet printing as a deposition and patterning tool for polymers and inorganic particles, Soft Matter. 4 (2008) 703713. 
[200] W. Han and Z. Lin, Learning from "Coffee Rings": Ordered Structures Enabled by Controlled Evaporative Self-Assembly, Angew. Chem. Int. Ed. 51 (2012) 1534-1546.

[201] T. Still, P.J. Yunker, and A.G. Yodh, Surfactant-induced Marangoni eddies alter the coffee-rings of evaporating colloidal drops, Langmuir. 28 (2012) 4984-4988.

[202] D. Soltman and V. Subramanian, Inkjet-Printed Line Morphologies and Temperature Control of the Coffee Ring Effect, Langmuir. 24 (2008) 2224-2231.

[203] X. Zhong, A. Crivoi, and F. Duan, Sessile nanofluid droplet drying, Adv. Colloid Interfac. 217 (2015) 13-30.

[204] F. Brochard-Wyart, R. Fondecave, and M. Boudoussier, Wetting of antagonist mixtures: the 'leak out' transition, Int. J. Eng. Sci. 38 (2000) 1033-1047.

[205] J.J. Cras, C.A. Rowe-Taitt, D.A. Nivens, and F.S. Ligler, Comparison of chemical cleaning methods of glass in preparation for silanization, Biosens. Bioelectron. 14 (1999) 683-688.

[206] S. Bharathi, N. Fishelson, and O. Lev, Direct Synthesis and Characterization of Gold and Other Noble Metal Nanodispersions in Sol-Gel-Derived Organically Modified Silicates, Langmuir. 15 (1999) 1929-1937.

[207] K. Aslan, P. Holley, and C.D. Geddes, Metal-enhanced fluorescence from silver nanoparticle-deposited polycarbonate substrates, J. Mater. Chem. 16 (2006) 28462852.

[208] N. Hajduková, M. Procházka, J. Štěpánek, and M. Špírková, Chemically reduced and laser-ablated gold nanoparticles immobilized to silanized glass plates: preparation, characterization and SERS spectral testing, Colloid. Surface. A. 301 (2007) 264-270.

[209] N. Nath and A. Chilkoti, Label-free biosensing by surface plasmon resonance of nanoparticles on glass: optimization of nanoparticle size, Anal. Chem. 76 (2004) $5370-8$.

[210] G. Chumanov, K. Sokolov, B.W. Gregory, and T.M. Cotton, Colloidal metal films as a substrate for surface-enhanced spectroscopy, J. Phys. Chem. 99 (1995) 9466-9471.

[211] I. Toshikazu, I. Yasushi, and T. Jiro, Nucleophilic Substitution of Aromatic Halides with Amines under High Pressure, Chem. Lett. 16 (1987) 1187-1190.

[212] M.F. Hughes, M. Menache, and D.J. Thompson, Dose-Dependent Disposition of Sodium Arsenate in Mice Following Acute Oral Exposure, Fundam. Appl. Toxicol. 22 (1994) 80-89. 
[213] G.K.H. Tam, S.M. Charbonneau, F. Bryce, and E. Sandi, Excretion of a single oral dose of fish-arsenic in man, Bull. Environ. Contam. Toxicol. 28 (1982) 669673.

[214] G.K.H. Tam, S.M. Charbonneau, G. Lacroix, and F. Bryce, Confirmation of inorganic arsenic and dimethylarsinic acid in urine and plasma of dog by ionexchange and TLC, Bull. Environ. Contam. Toxicol. 21 (1979) 371-374.

[215] S. Gibaud and G. Jaouen, Arsenic-based drugs: from fowler's solution to modern anticancer chemotherapy, Medicinal organometallic chemistry, in, Springer, pp. $1-20$.

[216] S.A. Stice, Speciation, Metabolism, Toxicity, and Protein-binding of Different Arsenic Species in Human Cells, edition ed, Florida International University, Miami. 1203. http://digitalcommons.fiu.edu/etd/1203.

[217] T.R. Radabaugh and H.V. Aposhian, Enzymatic reduction of arsenic compounds in mammalian systems: reduction of arsenate to arsenite by human liver arsenate reductase, Chem. Res. Toxicol. 13 (2000) 26-30.

[218] B.K. Mandal, Y. Ogra, and K.T. Suzuki, Identification of dimethylarsinous and monomethylarsonous acids in human urine of the arsenic-affected areas in West Bengal, India, Chem. Res. Toxicol. 14 (2001) 371-378.

[219] R. Xie, W. Johnson, S. Spayd, G.S. Hall, and B. Buckley, Arsenic speciation analysis of human urine using ion exchange chromatography coupled to inductively coupled plasma mass spectrometry, Anal. Chim. Acta. 578 (2006) 186-194.

[220] C.A. Loffredo, H.V. Aposhian, M.E. Cebrian, H. Yamauchi, and E.K. Silbergeld, Variability in human metabolism of arsenic, Environ. Res. 92 (2003) 85-91.

[221] B.K. Mandal, Y. Ogra, K. Anzai, and K.T. Suzuki, Speciation of arsenic in biological samples, Toxicol. Appl. Pharmacol. 198 (2004) 307-318.

[222] O.L. Valenzuela, V.H. Borja-Aburto, G.G. Garcia-Vargas, M.B. Cruz-Gonzalez, E.A. Garcia-Montalvo, E.S. Calderon-Aranda, and L.M. Del Razo, Urinary Trivalent Methylated Arsenic Species in a Population Chronically Exposed to Inorganic Arsenic, Environ. Health Persp. 113 (2005) 250-254.

[223] A. Raab, S.H. Wright, M. Jaspars, A.A. Meharg, and J. Feldmann, Pentavalent arsenic can bind to biomolecules, Angew. Chem. Int. Ed. 46 (2007) 2594-2597.

[224] L. Yehiayan, S. Stice, G. Liu, S. Matulis, L.H. Boise, and Y. Cai, Dimethylarsinothioyl Glutathione as a Metabolite in Human Multiple Myeloma Cell Lines upon Exposure to Darinaparsin, Chemical Research in Toxicology. 27 (2014) 754-764. 
[225] S. Stice, G. Liu, S. Matulis, L.H. Boise, and Y. Cai, Determination of multiple human arsenic metabolites employing high performance liquid chromatography inductively coupled plasma mass spectrometry, J. Chromatogr. B. 1009 (2016) $55-65$.

[226] N. Hua, R. Kanwal, L. X Chris, and D.J. Thomas, Formation of methylated oxyarsenicals and thioarsenicals in wild-type and arsenic (+3 oxidation state) methyltransferase knockout mice exposed to arsenate, Anal. Bioanal. Chem. 405 (2013) $1885-1891$.

[227] B. Chen, X. Lu, S. Shen, L.L. Arnold, S.M. Cohen, and X.C. Le, Arsenic Speciation in the Blood of Arsenite-Treated F344 Rats, Chem. Res. Toxicol. 26 (2013) 952-962.

[228] H. Naranmandura, N. Suzuki, K. Iwata, S. Hirano, and T. Suzuki, Arsenic Metabolism and Thioarsenicals in Hamsters and Rats, Chem. Res. Toxicol. 20 (2007) 616-624.

[229] R. Raml, A. Rumpler, W. Goessler, M. Vahter, L. Li, T. Ochi, and K.A. Francesconi, Thio-dimethylarsinate is a common metabolite in urine samples from arsenic-exposed women in Bangladesh, Toxicol. Appl. Pharmacol. 222 (2007) 374-380.

[230] T. Van de Wiele, C.M. Gallawa, K.M. Kubachka, J.T. Creed, N. Basta, E.A. Dayton, S. Whitacre, G. Du Laing, and K. Bradham, Arsenic metabolism by human gut microbiota upon in vitro digestion of contaminated soils, Environ. Health Persp. 118 (2010) 1004-1009.

[231] R. Raml, W. Goessler, P. Traar, T. Ochi, and K.A. Francesconi, Novel Thioarsenic Metabolites in Human Urine after Ingestion of an Arsenosugar, 2', $3^{6}-$ Dihydroxypropyl 5-Deoxy-5-dimethylarsinoyl- $\beta$-d-riboside, Chem. Res. Toxicol. 18 (2005) 1444-1450.

[232] R. Raml, W. Goessler, and K.A. Francesconi, Improved chromatographic separation of thio-arsenic compounds by reversed-phase high performance liquid chromatography-inductively coupled plasma mass spectrometry, J. Chromatogr. A. 1128 (2006) 164-170.

[233] K.T. Suzuki, B.K. Mandal, A. Katagiri, Y. Sakuma, A. Kawakami, Y. Ogra, K. Yamaguchi, Y. Sei, K. Yamanaka, K. Anzai, M. Ohmichi, H. Takayama, and N. Aimi, Dimethylthioarsenicals as Arsenic Metabolites and Their Chemical Preparations, Chem. Res. Toxicol. 17 (2004) 914-921.

[234] H. Naranmandura and K.T. Suzuki, Formation of dimethylthioarsenicals in red blood cells, Toxicol. Appl. Pharmacol. 227 (2008) 390-399. 
[235] H. Naranmandura, N. Suzuki, and K.T. Suzuki, Trivalent arsenicals are bound to proteins during reductive methylation, Chem. Res. Toxicol. 19 (2006) 1010-1018.

[236] G. Yang, X. Xu, W. Wang, L. Xu, G. Chen, and F. Fu, A new interface used to couple capillary electrophoresis with an inductively coupled plasma mass spectrometry for speciation analysis, Electrophoresis. 29 (2008) 2862-2868.

[237] G.-D. Yang, J.-P. Zheng, H.-X. Huang, G.-M. Qi, J.-H. Xu, and F.-F. Fu, Speciation Analysis of Arsenic in Seafood with Capillary Electrophoresis-UV Detection, Chin. J. Anal. Chem. 37 (2009) 532-536.

[238] G. Yang, J. Zheng, L. Chen, Q. Lin, Y. Zhao, Y. Wu, and F. Fu, Speciation analysis and characterisation of arsenic in lavers collected from coastal waters of Fujian, south-eastern China, Food Chem. 132 (2012) 1480-1485.

[239] L. Yehiayan, M. Pattabiraman, K. Kavallieratos, X. Wang, L.H. Boise, and Y. Cai, Speciation, formation, stability and analytical challenges of human arsenic metabolites, Journal of Analytical Atomic Spectrometry. 24 (2009) 1397-1405.

[240] E.B. Hanlon, R. Manoharan, T.W. Koo, K.E. Shafer, J.T. Motz, M. Fitzmaurice, J.R. Kramer, I. Itzkan, R.R. Dasari, and M.S. Feld, Prospects for in vivo Raman spectroscopy, Phys. Med. Biol. 45 (2000) R1-R59.

[241] J. Gailer, Arsenic-selenium and mercury-selenium bonds in biology, Coord. Chem. Rev. 251 (2007) 234-254.

[242] X. Qian, X.H. Peng, D.O. Ansari, Q. Yin-Goen, G.Z. Chen, D.M. Shin, L. Yang, A.N. Young, M.D. Wang, and S. Nie, In vivo tumor targeting and spectroscopic detection with surface-enhanced Raman nanoparticle tags, Nat. Biotechnol. 26 (2008) 83-90.

[243] G. Trachta, B. Schwarze, B. Sägmüller, G. Brehm, and S. Schneider, Combination of high-performance liquid chromatography and SERS detection applied to the analysis of drugs in human blood and urine, J. Mol. Struct. 693 (2004) 175-185.

[244] J. Lin, R. Chen, S. Feng, J. Pan, Y. Li, G. Chen, M. Cheng, Z. Huang, Y. Yu, and $\mathrm{H}$. Zeng, A novel blood plasma analysis technique combining membrane electrophoresis with silver nanoparticle-based SERS spectroscopy for potential applications in noninvasive cancer detection, Nanomed. Nanotechnol. Biol. Med. 7 (2011) 655-663.

[245] S. Feng, R. Chen, J. Lin, J. Pan, G. Chen, Y. Li, M. Cheng, Z. Huang, J. Chen, and H. Zeng, Nasopharyngeal cancer detection based on blood plasma surfaceenhanced Raman spectroscopy and multivariate analysis, Biosens. Bioelectron. 25 (2010) 2414-2419. 
[246] A. Rogstad, High-temperature laser Raman spectroscopic study on mixtures of arsenic and sulphur vapours, J. Mol. Struct. 14 (1972) 421-426.

[247] W. Mikenda, H. Steidl, and A. Preisinger, Raman spectra of Na3AsS4·8(D, H)2O and $\mathrm{Na} 3 \mathrm{SbS} 4 \cdot 9(\mathrm{D}, \mathrm{H}) 2 \mathrm{O}$ and $\mathrm{OD}(\mathrm{H}) \ldots \mathrm{S}$ bonds in salt hydrates, J. Raman Spectrosc. 12 (1982) 217-221.

[248] E.E. Zvereva, A.R. Shagidullin, and S.A. Katsyuba, Ab initio and DFT predictions of infrared intensities and Raman activities, J. Phys. Chem. A. 115 (2011) 63-69.

[249] G.R. Helz, J.A. Tossell, J.M. Charnock, R.A.D. Pattrick, D.J. Vaughan, and C.D. Garner, Oligomerization in As (III) sulfide solutions: Theoretical constraints and spectroscopic evidence, Geochim. Cosmochim. Acta. 59 (1995) 4591-4604.

[250] M.C. Teixeira, V.S.T. Ciminelli, M.S.S. Dantas, S.F. Diniz, and H.A. Duarte, Raman spectroscopy and DFT calculations of As(III) complexation with a cysteine-rich biomaterial, J. Colloid. Interf. Sci. 315 (2007) 128-134.

[251] J.A. Tossell and M.D. Zimmermann, Calculation of the structures, stabilities, and vibrational spectra of arsenites, thioarsenites and thioarsenates in aqueous solution, Geochim. Cosmochim. Acta. 72 (2008) 5232-5242.

[252] M. Fricke, M. Zeller, W. Cullen, M. Witkowski, and J. Creed, Dimethylthioarsinic anhydride: a standard for arsenic speciation, Anal. Chim. Acta. 583 (2007) 78-83.

[253] M.W. Fricke, M. Zeller, H. Sun, V.W.-M. Lai, W.R. Cullen, J.A. Shoemaker, M.R. Witkowski, and J.T. Creed, Chromatographic separation and identification of products from the reaction of dimethylarsinic acid with hydrogen sulfide, Chem. Res. Toxicol. 18 (2005) 1821-1829.

[254] N. Garnier, G.G.J. Redstone, M.S. Dahabieh, J.N. Nichol, S.V. del Rincon, Y. Gu, D.S. Bohle, Y. Sun, D.S. Conklin, K.K. Mann, and W.H. Miller, The Novel Arsenical Darinaparsin is Transported by Cystine Importing Systems, Mol. Pharmacol. (2014) 576-585.

[255] X. Xu and D.G. Truhlar, Accuracy of effective core potentials and basis sets for density functional calculations, including relativistic effects, as illustrated by calculations on arsenic compounds, J. Chem. Theory Comput. 7 (2011) 27662779 .

[256] F. Weigend and R. Ahlrichs, Balanced basis sets of split valence, triple zeta valence and quadruple zeta valence quality for $\mathrm{H}$ to $\mathrm{Rn}$ : design and assessment of accuracy, Phys. Chem. Chem. Phys. 7 (2005) 3297-3305. 
[257] P.B. Armentrout and I. Kretzschmar, Experimental and Theoretical Studies of the Reaction of $\mathrm{Rh}+$ with CS2 in the Gas Phase: Thermochemistry of $\mathrm{RhS}+$ and RhCS+, J. Phys. Chem. A. 113 (2009) 10955-10965.

[258] G. Schaftenaar and J.H. Noordik, Molden: a pre-and post-processing program for molecular and electronic structures, J. Comput.Aid. Mol. Des. 14 (2000) 123-134.

[259] T. Lu and F. Chen, Multiwfn: a multifunctional wavefunction analyzer, J. Comput. Chem. 33 (2012) 580-592.

[260] A.P. Scott and L. Radom, Harmonic vibrational frequencies: an evaluation of Hartree-Fock, Møller-Plesset, quadratic configuration interaction, density functional theory, and semiempirical scale factors, J. Phys. Chem. 100 (1996) $16502-16513$.

[261] R.F. Hout, B.A. Levi, and W.J. Hehre, Effect of electron correlation on theoretical vibrational frequencies, J. Comput. Chem. 3 (1982) 234-250.

[262] J.A. Pople, H.B. Schlegel, R. Krishnan, D.J. Defrees, J.S. Binkley, M.J. Frisch, R.A. Whiteside, R.F. Hout, and W.J. Hehre, Molecular orbital studies of vibrational frequencies, Int. J. Quantum Chem. 20 (1981) 269-278.

[263] H.-V. Gründler, H.-D. Schumann, and E. Steger, Raman and infrared spectroscopic investigation of alkyl derivatives of arsenic acid, J. Mol. Struct. 21 (1974) 149-157.

[264] I. Silaghidumitrescu, L. Silaghidumitrescu, And I. Haiduc, Normal Coordinate Analysis Of The Vibrational-Spectrum Of Dimethyldithioarsinato Anion,(CH3)2AsS2-, Rev Roum. Chim. 27 (1982) 911-916.

[265] L.S. Dumitrescu, I. Haiduc, and J. Weiss, Preparation and properties of some organotin dimethyl- and diphenyl-dithioarsinates. The crystal structure of (CH3)2Sn[S2As(CH3)2]2, J. Organomet. Chem. 263 (1984) 159-165.

[266] I. Haiduc and L. Silaghi-Dumitrescu, Organotin and tin(IV) derivatives of dimethyldithioarsinic acid, Journal of Organometallic Chemistry. 225 (1982) 225232.

[267] J. De Gelder, K. De Gussem, P. Vandenabeele, and L. Moens, Reference database of Raman spectra of biological molecules, J. Raman Spectrosc. 38 (2007) 11331147.

[268] K.B. Wiberg, Basis set effects on calculated geometries: 6-311++ $\mathrm{G}^{* *}$ vs. aug-ccpVDZ, J. Comput. Chem. 25 (2004) 1342-1346.

[269] P. Sinha, S.E. Boesch, A. Changming Gu, R.A. Wheeler, and A.K. Wilson, Harmonic Vibrational Frequencies: Scaling Factors for HF, B3LYP, and MP2 
Methods in Combination with Correlation Consistent Basis Sets, J. Phys. Chem. A. 108 (2004) 9213-9217.

[270] G.E. Walrafen, Raman Spectral Studies of Aqueous Solutions of Selenic Acid, J. Chem. Phys. 39 (1963) 1479-1492.

[271] M. Falk and P.A. Giguère, INFRARED SPECTRA AND STRUCTURE OF SELENIOUS ACID, Can. J. Chem. 36 (1958) 1680-1685.

[272] M.W. Wong, Vibrational frequency prediction using density functional theory, Chem. Phys. Lett. 256 (1996) 391-399.

[273] R.A. Zingaro, K.J. Irgolic, D.H. O'Brien, and L.J. Edmonson, Rearrangement of tetramethyldiarsine disulfide, J. Am. Chem. Soc. 93 (1971) 5677-5681.

[274] W.R. Cullen, G.B. Deacon, and J.H.S. Green, VIBRATIONAL SPECTRA OF SOME ALKYLARSONIUM COMPOUNDS, Can. J. Chem. 43 (1965) 31933200 .

[275] A Relation between Bond Force Constants, Bond Orders, Bond Lengths, and the Electronegativities of the Bonded Atoms, J. Chem. Phys. 14 (1946) 305-320.

[276] I. Haiduc and L. Silaghi-Dumitrescu, Organotin and tin (IV) derivatives of dimethyldithioarsinic acid, J. Organomet. Chem. 225 (1982) 225-232.

[277] A. Casey, N. Ham, D. Mackey, and R. Martin, Synthesis and infrared spectra of some dithiocacodylate complexes, Aust. J. Chem. 23 (1970) 1117-1123.

[278] V. Tkachev, V. Perov, and Y.F. Gatilov, Crystal and molecular structure of dipropylphenylarsine sulfide (C 3 H 7) 2 (C 6 H 5) AsS, J Struct. Chem. 25 (1984) 144-146.

[279] R.A. Zingaro, R.E. McGlothin, and R.M. Hedges, Infra-red absorption spectra of eleven arsine sulphides from 15 to 30 [small micro]. The as-S stretching frequency, T. Faraday Soc. 59 (1963) 798-805. 
VITA

\section{MINGWEI YANG}

2005-2009

B.S., Chemistry

Fuzhou University

Fujian, China

2009-2012

M.S., Environmental Chemistry

Excellent Master Thesis Award

Fuzhou University

Fujian, China

$2012-2017$

Doctoral Candidate, Chemistry

Florida International University

Miami, Florida

Teaching Assistant

Florida International University

Miami, Florida

\section{PUBLICATIONS AND PRESENTATIONS}

Yang, M. W.; Wang, Z. W.; Fang, L.; Zheng, J. P.; Xu, L. J.; Fu, F. F., Simultaneous and ultra-sensitive quantification of multiple peptides by using europium chelate labeling and capillary electrophoresis-inductively coupled plasma mass spectrometry. J. Anal. Atom. Spectrom. 2012, 27 (6), 946-951.

Yang, M. W..; Wu, W.; Ruan, Y.; Huang, L.; Wu, Z.; Cai, Y.; Fu, F., Ultra-sensitive quantification of lysozyme based on element chelate labeling and capillary electrophoresis-inductively coupled plasma mass spectrometry. Anal. Chim. Acta 2014, $812,12-17$.

Jiang, W.; Cai, Q.; Xu, W.; Yang, M. W.; Cai, Y.; Dionysiou, D. D.; O'Shea, K. E., $\mathrm{Cr}(\mathrm{VI})$ adsorption and reduction by humic acid coated on magnetite. Environ. Sci. Technol. 2014, 48 (14), 8078-85.

Yang, M. W.; Matulis, S.; Boise, L. H.; McGoron, A. J.; Cai, Y., Potential application of SERS for arsenic speciation in biological matrices, Anal. Bioanal. Chem., accepted as forefront article on May 26, 2017

Yang, M. W.; Sun, Y. Z.; Zhang, X. B.; McCord, B.; McGoron, A. J.; Mebel, A.; Cai, Y., Raman spectra of thiolated arsenicals with biological importance, under preparation 
Yang, M. W.; Fan, C. J.; Sylvers, K.; McGoron, A. J.; Liu, G. L.; Cai, Y., Employing coffee ring effect on silver nanofilm for arsenic speciation study, under preparation

$91^{\text {st }}$ Florida Annual Meeting and Exposition, 5/2015, Tampa, FL (poster)

2017 Florida International University Graduate Student Appreciation Week, 3/2017, Miami, FL (poster) 